\title{
Mechanical properties of pore-spanning membranes prepared from giant vesicles
}

\author{
Doctoral thesis \\ In partial fulfillment of the requirements for the degree \\ Doctor of Philosophy \\ Ph.D. \\ Division of Mathematics and Natural Sciences \\ of the Georg-August-Universität Göttingen
}

submitted by

Marta Kocun

Göttingen 2011 


\section{Members of the Thesis committee}

Prof. Dr. Andreas Janshoff (research supervisor)

Institute for Physical Chemistry

Georg-August University Göttingen

Prof. Dr. Ulf Diederichsen

Institute for Organic and Biomolecular Chemistry

Georg-August University Göttingen

Dr. Iwan Schaap

$3^{\text {rd }}$ Physics Institute, Faculty of Physics

Georg-August University Göttingen

Date of the oral examination: May $23^{\text {rd }} 2011$ 
To my Mother and Brother

The more you read, The more things you will know.

The more you learn, The more places you'll go.

- Dr. Seuss 
I, Marta Kocun, hereby certify that my doctoral thesis entitled "Mechanical properties of pore-spanning membranes prepared from giant vesicles" has been written independently and with no other sources and aids than quoted.

Marta Kocun

April $15^{\text {th }} 2011$

Göttingen, Germany 


\section{TABLE OF CONTENTS}

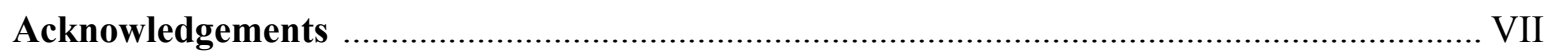

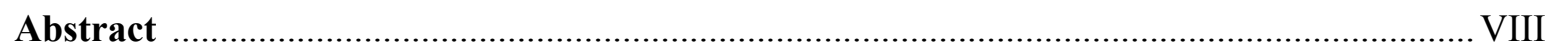

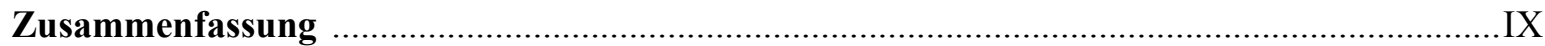

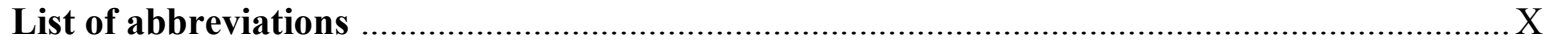

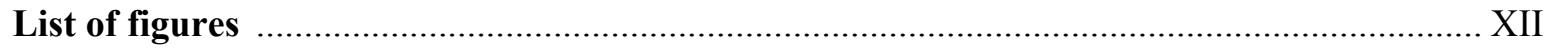

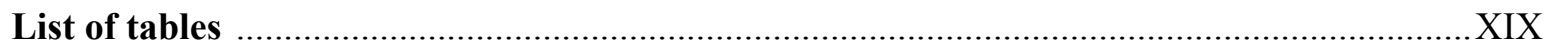

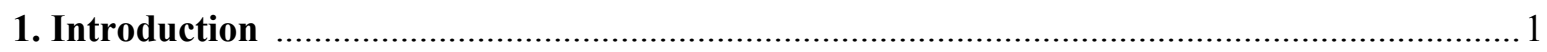

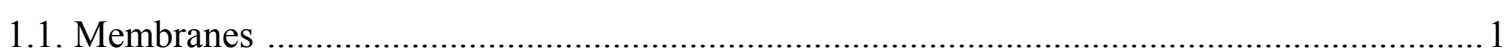

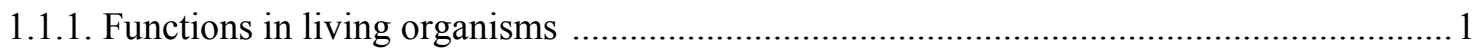

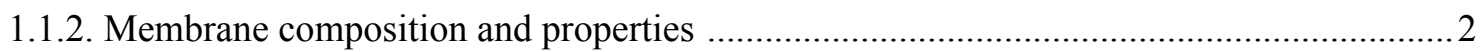

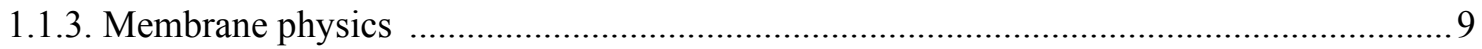

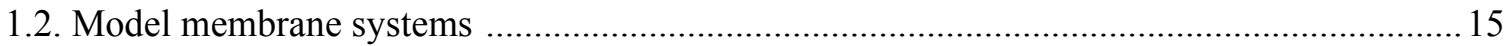

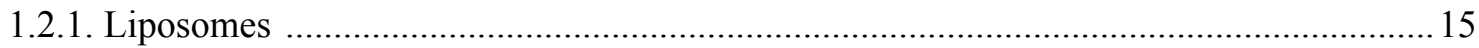

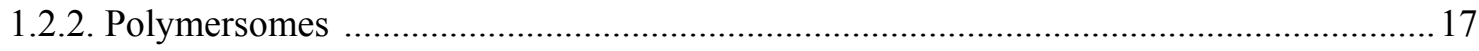

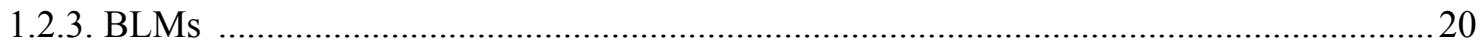

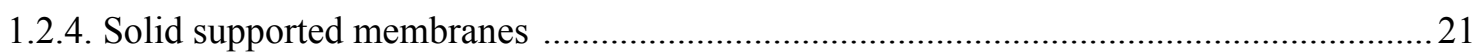

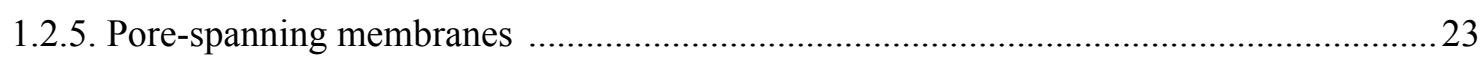

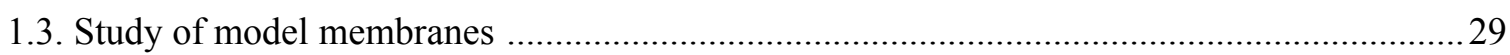

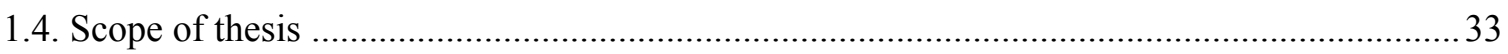

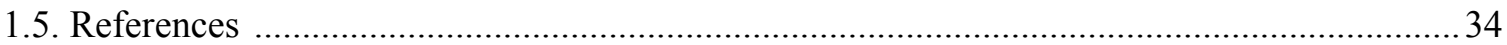

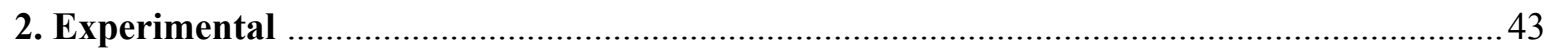

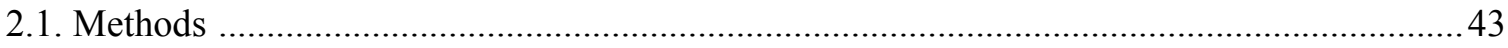

2.1.1. Optical microscopy: white light and fluorescence microscopy ......................................43

2.1.2. Scanning probe microscopy: atomic force microscopy (AFM) ...................................... 46

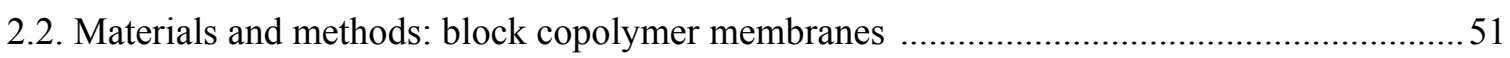

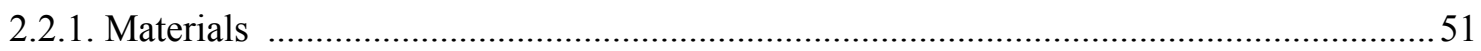

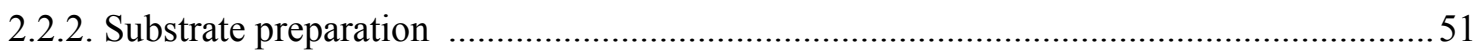

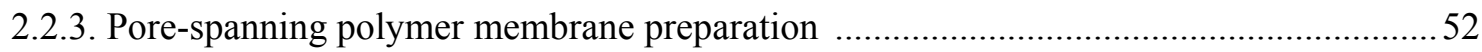

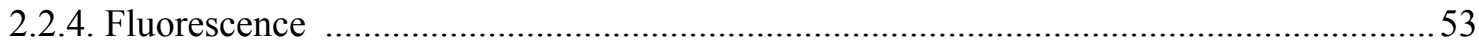

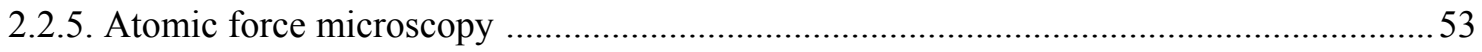

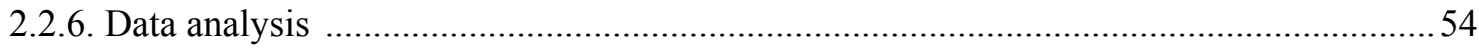

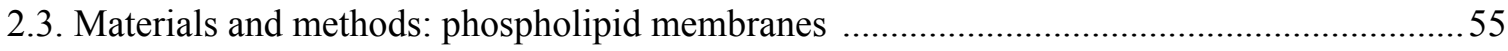

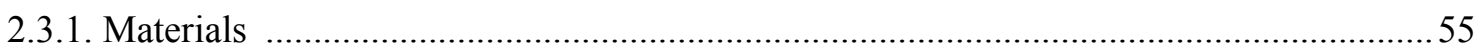

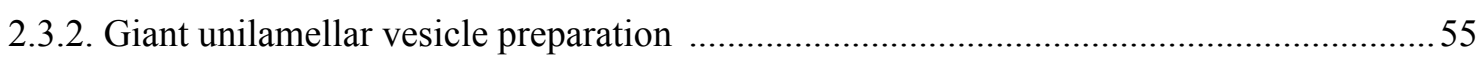




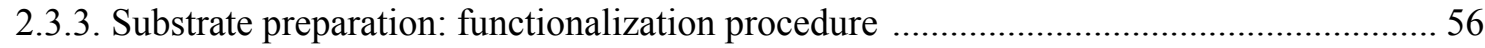

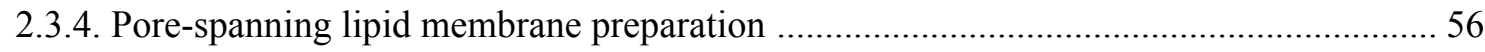

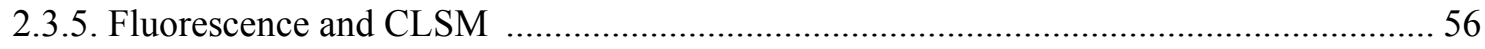

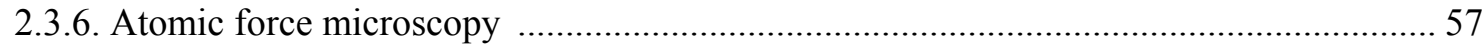

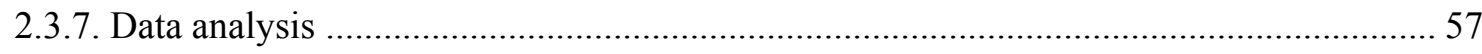

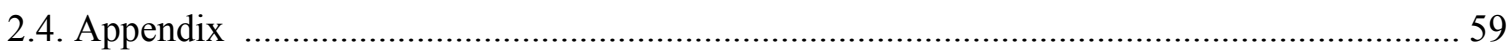

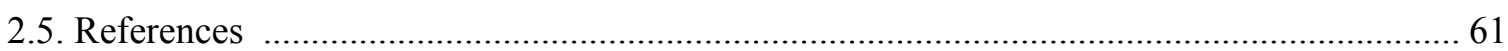

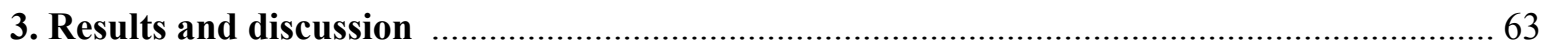

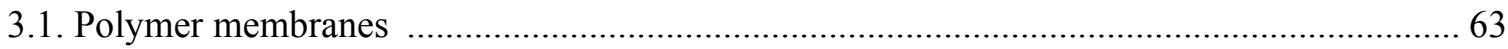

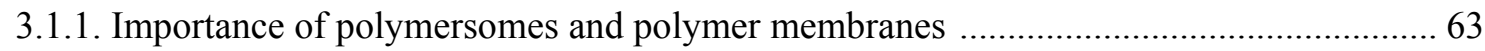

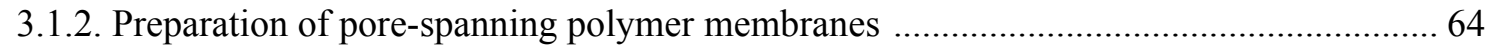

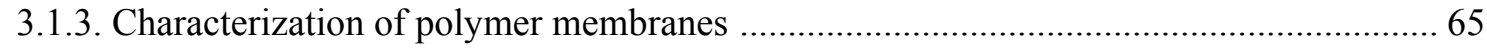

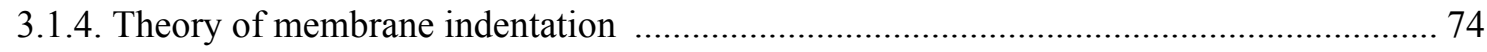

3.1.5. Force spectroscopy study of pore-spanning polymer membranes .................................. 78

3.1.6. Mechanical properties of polymer pore-spanning membranes ..................................... 87

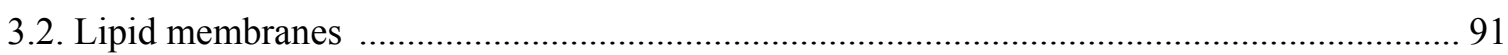

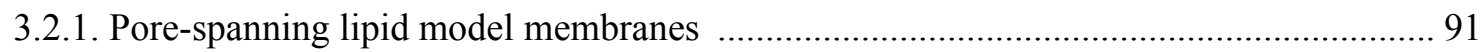

3.2.2. Preparation of pore-spanning lipid membranes ............................................................... 92

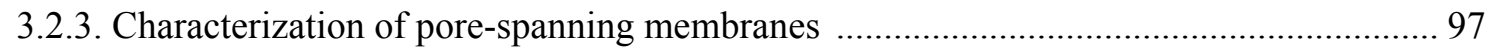

3.2.4. Force spectroscopy study of pore-spanning lipid membranes ....................................... 109

3.2.5. Force volume experiments on pore-spanning membranes .......................................... 114

3.2.6. Determination of bending modulus through tether analysis ......................................... 119

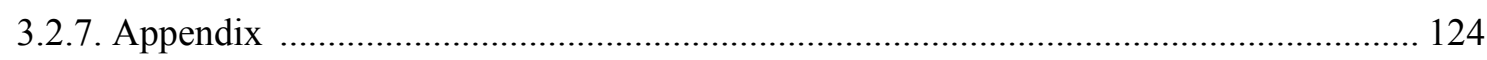

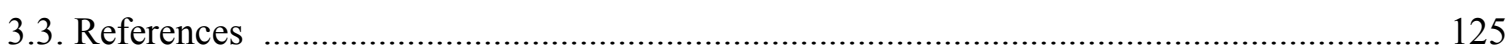

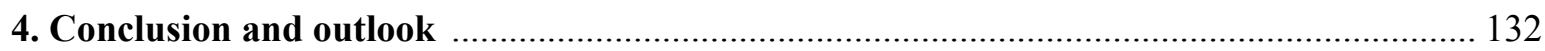

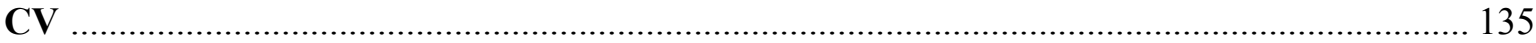




\section{ACKNOWLEDGEMENTS}

I gratefully acknowledge the funding of my work by the International Max Planck Research Scholarships (Mainz and Göttingen), as well as the travel grants and bridging fund from the Göttingen Graduate School for Neurosciences and Molecular Biosciences (GGNB).

I would like to thank my research supervisor, Prof. Andreas Janshoff, for giving me the opportunity to work in his research lab, for his guidance, his positive attitude and energetic approach to research. I must say that final twists on unfinished projects and the most excellent advice in times of confusion were truly appreciated.

I thank my thesis committee members, Prof. Ulf Diederichsen and Dr. Iwan Schaap for taking time to follow my research progress during the last two years, their support and scientific advice.

I also thank Prof. Claudia Steinem for her help, valuable research input and support.

I also thank: Dr. Waltraut Muller and Dr. Michael Maskos for providing polymersomes used in this work as well as for their research input. Holger Adam, for introducing me to the instruments and those great lengthy Igor procedures. Dr. Matthias Janke, for sharing many valuable points about research and most importantly his social commentaries on the inner workings of the Janshoff research group. Members of the Janshoff and Steinem research groups for numerous outings, activities, conference travels and exciting working environment.

Ich danke Frau Lappe für ihre Hilfe mit all the contracts, phone calls and letters in german.

Gen and Charlie, for fun vacations while exploring Germany, Czech Republic and Portugal!

I also wish to thank my family and friends who, from far away, continuously encouraged me to work hard and embrace diversity.

Finally, I want to thank Thomas without who I could not have accomplished this work. By valuing my strengths and accepting my weaknesses, by offering uplifting words, advice and love you helped me achieve my aspirations. During the last three years, your presence made rainy days seem short and sunny ones were enjoyed to their fullest. Thank you for always being there for me, for sharing your enthusiasm, curiosity, amazing travels and many laughs! 


\section{ABSTRACT}

Research interest in the mechanical properties of membranes arises from the multitude of morphological and physiological functions they govern. However, the complexity of native membranes requires the use of model systems which mimic the behavior of biological membranes. Here, pore-spanning membranes, which combine the free-standing character of a black lipid membrane together with the stability of a solid-supported membrane, have been prepared on porous substrates. Fluorescence microscopy, atomic force microscopy (AFM) imaging and force indentation experiments performed on these pore-spanning membranes provided information about their mechanical properties. Porespanning polymer membranes were prepared from poly(butadiene)- $b$-poly(ethylene oxide) polymersome rupture induced by osmotic stress. The pore-spanning polymer membranes were studied by force indentation experiments and were found to exhibit viscoelastic behavior. The energy dissipation was quantified through calculation of the extent of hysteresis observed between indentation and retraction force curves. UV light cross-linking of the hydrophobic poly(butadiene) core of the polymer membranes was performed to study the effect of chain entanglement on their viscoelastic properties. Furthermore, two-step functionalization procedure was developed to prepare solvent-free, laterally mobile, robust pore-spanning phospholipid membranes by giant phospholipid vesicle rupture. Force indentation experiments provided membrane tension values, while preliminary membrane bending rigidity values were calculated from force needed to pull tethers out of pore-spanning membranes upon AFM tip retraction. Force volume imaging of several pores in a single experiment demonstrated the robustness of the pore-spanning membranes and the efficiency of AFM force indentation experiments. Subtle changes in the mechanical properties of the pore-spanning membranes were observed after addition of isopropanol to the membrane environment which confirmed the sensitivity of the investigation approach proposed in this work. 


\section{ZUSAMMENFASSUNG}

Das wissenschaftliche Interesse an den mechanischen Eigenschaften von Membranen erwächst aus deren vielfältigen morphologischen und physiologischen Funktionen. Jedoch erfordert die Komplexität nativer Membranen die Verwendung von Modellsystemen, die das Verhalten biologischer Membranen nachahmen. In dieser Arbeit wurden porenüberspannende Membranen, welche die freitragenden Eigenschaften schwarzer Lipidmembranen (Black Lipid Membranes, BLM) sowie die Stabilität festkörperunterstützter Membranen kombinieren, auf porösen Substraten verwendet.Die mechanischen Eigenschaften dieser porenüberspannenden Membranen wurden mit Fluoreszenzmikroskopie und sowohl abbildener Rasterkraftmikroskopie (AFM) als auch Indentationsexperimenten untersucht.Porenüberspannende Polymermembranen wurden aus Poly(butadien)- $b$-poly(ethylenoxid)-Polymerosomen durch osmotisch induziertes Spreiten hergestellt. Die porenüberspannenden Polymermembranen wurden mit Kraftindentationsexperimenten untersucht, wobei sie ein viskoelastisches Verhalten zeigten. Die Energiedissipation wurde durch Berechnungen der Hysterese zwischen Indentations- und Abrisskraftkurve erhalten. UV-induzierte Quervernetzung des hydrophoben Poly(butadien)-Kerns der Polymermembran diente zur Untersuchung des Einflusses des Verzweigungsgrades der Ketten auf ihre viskoelastischen Eigenschaften. Darüber hinaus wurde ein zweistufiges Funktionalisierungsprotokoll entwickelt um lösungsmittelfreie, lateral bewegliche, stabile porenüberspannende Phsospholipidmembranen durch Spreiten großer Phospholipidvesikel zu erhalten.Kraftindentationsexperimente lieferten Membranspannungswerte, während Membrankrümmungssteifigkeiten aus der Zugkraft erhalten wurden, welche benötigt wird um beim Rückzug des AFM Cantilevers Tether aus porenüberspannenden Membranen zu ziehen.

Force Mapping von mehreren Poren während einem einzelnen Experiment demonstrierte die Stabilität der porenüberspannenden Membranen und die Effizienz der AFMKraftindentationsexperimente. Geringfügige Änderungen der viskolelastischen Eigenschaften der porenüberspannenden Membranen wurden nach der Zugabe von Isopropanol zur Membranumgebung beobachtet, was die Sensitivität der gewählten Untersuchungsmethode in dieser Arbeit bestätigt. 


\section{LIST OF ABBREVIATIONS}

A

$\mathrm{A}_{\mathrm{f}}$

AFM

B

BLM

$\mathrm{C}_{0}$

CLSM

D

$d$

$d_{\mathrm{c}}$

DOPC

DPhPC

E

$f$

$F_{\mathrm{c}}$

$F_{\mathrm{m}}$

$F_{\max }$

FRAP

$\gamma$

GUV

$\eta$

$\eta_{\text {eff }}$

$h$

I

ITO

$k$

$\kappa$

$K_{\mathrm{a}}$

$k_{\text {app }}$

$k_{\mathrm{B}}$

Area

Attenuation factor of the laser beam

Atomic Force Microscopy

Correction factor associated with optical lever method

Black Lipid Membrane

Initial fluorophore concentration

Confocal Laser Scanning Microscpy

Diffusion constant

Membrane thickness

Cantilever deflection

1,2-dioleoyl-sn-glycero-3-phosphocholine

1,2-diphytanoyl-sn-glycero-3-phosphocholine

Young's modulus

Hydrophilic volume fraction

Cantilever force

Membrane force

Maximal indentation force

Fluorescence Recovery After Photobleaching

Surface tension

Giant Unilamellar Vesicle

Surface shear viscosity

Effective surface viscosity

Indentation depth

Intensity

Indium Tin Oxide

Apparent spring constant

Bending modulus

Area compressibility

Apparent spring constant of membrane

Boltzmann constant 


\begin{tabular}{|c|c|}
\hline$k_{\mathrm{c}}$ & Cantilever spring constant \\
\hline$k_{\mathrm{m}}$ & Nonlinear spring \\
\hline M & Molar \\
\hline MFP & Molecular Force Probe \\
\hline $\mathrm{mL}$ & Mililiter \\
\hline$\mu$ & Shear modulus \\
\hline$\mu \mathrm{m}$ & Micrometer \\
\hline $\mathrm{N}$ & Newton \\
\hline $\mathrm{nm}$ & Nanometer \\
\hline $\mathrm{P}$ & Packing parameter \\
\hline $\mathrm{P}_{0}$ & Total laser power \\
\hline PB- $b$-PEO & Poly(butadiene)-block-Poly(ethylene oxide) \\
\hline PBS & Phosphate buffered saline \\
\hline $\mathrm{pN}$ & Piconewton \\
\hline POPC & 1-palmitoyl-2-oleoyl-sn-glycero-3-phosphocholine \\
\hline $\mathrm{R}, \mathrm{r}$ & Radius \\
\hline ROI & Region of interest \\
\hline $\mathrm{s}$ & Second \\
\hline SAM & Self-Assembled Monolayer \\
\hline SEM & Scanning Electron Microscopy \\
\hline$\sigma_{\text {pre }}$ & Pre-tension \\
\hline $\mathrm{T}$ & temperature \\
\hline $\mathrm{t}$ & time \\
\hline $\mathrm{v}$ & velocity \\
\hline $\mathrm{V}$ & Volume \\
\hline$v$ & Poisson ratio \\
\hline$\sigma$ & Lateral tension \\
\hline$\tau$ & Relaxation time \\
\hline
\end{tabular}




\section{LIST OF FIGURES}

Figure 1.1 Schematic representation of membrane functions. In (a), membrane acts as a boundary between the extracellular matrix and the cytoplasm. Organelles are compartmentalization examples. (b) Oxidative phosphorylation in the mitochondrion where the membrane acts as a scaffold for chemical activity. (c) Channels embedded in the membrane allow for membrane transport and action potential generation. (d) Intercellular recognition through glycolipids present in the cellular membrane. p. 2

Figure 1.2 (a) Chemical structure of a phospholipid with a schematic representation of the phospholipid hydrophilic head and hydrophobic tails. (b) Chemical structures of the most common alcohol head groups of phospholipids. p. 3

Figure 1.3 (a) Schematic representation of phospholipid assembly to form a bilayer structures with hydrophobic tails forming the membrane core and the heads interacting with the aqueous environment. (b) A profile obtained from X-ray and neurton diffraction data showing the distribution of structural groups in a DOPC bilayer as a function of distance from the hydrocarbon core (HC). Figure taken from White et al. ${ }^{4}$ p. 4

Figure 1.4 Packing parameter $P$ which is the ratio of the volume of the hydrophobic chain $\mathrm{V}$ to the area ao and the maximal length of the hydrophobic chain $l_{\mathrm{c}}$ and predicts the structure of the aggregates formed from amphiphiles placed in aqueous solution. p. 6

Figure 1.5 Chemical structures of (a) cholesterol and (b) galactocerebroside, the simplest glycolipid with a galactose sugar residue. p. 7

Figure 1.6 Schematic representation of (a) transmembrane protein and (b) peripheral membrane protein p. 8

Figure 1.7 Relationship between the energy and the mean interface area a of an amphiphile at the water air interface. At high density, the energy increase with increasing packing of phospholipids; at low density, the energy increases with exposure of hydrocarbon tails. $a_{0}$ is the optimal interface area of an amphiphile. p. 11

Figure 1.8 During bilayer bending, the amphiphiles in the upper leaflet are pulled apart while the amphiphiles in the bottom leaflet are compressed. p. 12

Figure 1.9 Formation of liposomes from flat phospholipid bilayer films. The films are hydrated and the liposomes are created by swelling and closing of bilayer on themselves (adapted from Walde et al.$^{43}$ ). p. 16

Figure 1.10 Schematic representation of (a) block copolymer chain, (b) two types of repeating monomer units composing the polymer, (c) chemical structure of poly(butadiene)-block-poly(ethylene oxide) block copolymer and (d) various types of copolymers composed of two different monomers A and B: (1) alternating copolymer, (2) statistical copolymer, (3) block copolymer and (4) graft copolymer. p. 17

Figure 1.11 Relationship between the hydrophilic fraction $f$ of block copolymers and the structures formed from the aggregation of given block copolymers. (a) when $f=25-45 \%$ block copolymer has a cylindrical shape and vesicles are formed, (b) when $f>45 \%$ the block copolymer has a truncated cone shape and cylindrical vesicles are the preferred geometry and (c) when $f>50 \%$ the block copolymer assembles into micelles. In (d), the relationship between the increasing molecular weight of the polymer and the membrane thickness of polymersome is shown. (Figure adapted from Discher et al. ${ }^{62}$ ) p. 18

Figure 1.12 Schematic representation of a black lipid membrane (BLM) setup. A phospholipid bilayer is formed over a small aperture in a Teflon wall separating two chambers filled with aqueous solution. $\quad$ p. 20 
Figure 1.13 Schematic representation of (a) solid supported membrane where the phospholipid bilayer rests on a hydration layer of water molecules ${ }^{93,101}$ on the hydrophilic substrate and in (b) a solid supported hybrid membrane where a self-assembled monolayer of hydrophobic thiol molecules replaces the lower leaflet of the phospholipid bilayer. p. 22

Figure 1.14 Schematic representation showing a transmembrane protein within: (a) solid supported membrane, (b) polymer-cushioned membrane, (c) polymer-tethered membrane (d) hybrid membrane prepared on hydrophobic self-assembled monolayer. p. 23

Figure 1.15 Schematic representation of a hybrid pore-spanning membrane. Octanethiol SAM forms on the interpore surface and on the edge of the pore. Phospholipids painted over the porous surface arrange during the thinning process to create a bilayer membrane over the pore and a hybrid membrane on the pore-rim. p. 25

Figure 1.16 Experimental techniques used for investigation of mechanical properties of surfaces. (a) surface force apparatus technique suitable for solid-supported membranes (b) micropipette aspiration techniques used to investigate vesicles and (c) atomic force microscopy which can be used to investigate pore-spanning membranes. p. 29

Figure 2.1 (a) Schematic setup of a confocal fluorescent microscope. (b) Chemical structures of dye molecules which can undergo excitation and emission to create fluorescence. p. 44

Figure 2.2 Schematic representation of a FRAP experiment showing fluorescence intensity as a function of time. The initial fluorescence intensity $\left(\mathrm{I}_{\mathrm{i}}\right)$ of a region of interest (ROI) is shown in the graph as $a$ and as $a$, in the drawing of the sample. As ROI is photobleached ( $b$ in the graph and $b^{\prime}$ in the drawing), the fluorescence intensity drops to zero $\left(\mathrm{I}_{0}\right)$. When diffusion is possible, fluorescence intensity recovery takes place ( $c, d$ in the graph and $c^{\prime}, d^{\prime}$ in the drawing) and ROI regains almost all of its intensity $\left(\mathrm{I}_{\mathrm{r}}\right) . \quad$ p. 45

Figure 2.3 (a) AFM probe, (b) cantilevers, (c) sharp AFM tip mounted at the end of a cantilever, (d) a close-up view of the AFM tip with approximately $50 \mathrm{~nm}$ tip radius $r$ (shown in the inset). p. 46

Figure 2.4 (a) Energy potential diagram and (b) AFM imaging modes: contact mode (top) and intermittent contact mode (bottom). p. 47

Figure 2.5 Schematic representation of a force curve performed on a hard surface. On the left, data is presented in its raw form: detector signal versus piezo position. After determining the sensitivity of the detector and calculating the spring constant of the cantilever used to obtain the force curve, a force versus distance curve is determined. p. 48

Figure 2.6 (a) A photograph of six, square, fluxxion substrates where the colored area corresponds to pores. (b) Schematic representation of 14 lines of 10 porous areas found in each substrate. (c) Light microscope image of the first porous area and of the FLUXX SIEVE 1.20 writing which serves as a Cartesian coordinate of $(0,0)$. (d) Close-up of the flat (brighter) and porous (darker) areas on the substrate. (e) Closeup of porous area shown in d, (f) SEM image of the silicon nitride porous film. p. 52

Figure 3.1 (a) Poly(butadiene)-b-poly(ethylene oxide) (PB-b-PEO) block copolymer chemical structure and its schematic representation. The subscripts $a$ and $b$ indicate the respective number of copolymer blocks: $a=130$ and $b=66$. (b) Schematic representation of a polymersome loaded with a lipophilic Nile Red Dye and the arrangement of the block copolymer forming the polymeric membrane, (c) cyroTEM of PB-b-PEO polymersomes, (d) CLSM image of a polymersome containing smaller polymersome inside of it and (e) schematic representation of a pore-spanning polymer membrane. p. 64

Figure 3.2 (a) Epifluorescence of pore-spanning polymer membranes prepared by PB- $b$-PEO polymersome swelling and rupture. In addition to locating membrane patches, fluorescence images show if the patches 
are uniform or if excess material is covering the patch (excess material is indicated by arrows). (b) Close-up of the porous substrate covered with a polymer membrane. p. 65

Figure 3.3 (a) CLSM image of a polymer membrane spanning a few pores with an overlay of fluorescence intensity profile obtained from the line drawn along the pores (b) z-direction profile of 12 pores where only pores 3,4,5 and 6 are covered with polymer membrane. Scale bar figure $a$ : $5 \mu \mathrm{m}$, figure $b$ (height of arrow): $2 \mu \mathrm{m} \quad$ p. 67

Figure 3.4 (a) An orthogonal section view of a polymer vesicle docked on a porous substrate with the 3D image below (scale bar: 5um). (b) An orthogonal section view of a deflated vesicle on a porous surface with a 3D image below (scale bar: $5 \mu \mathrm{m}$ ). p. 68

Figure 3.5 (a) Schematic representation of a polymersome with an enlargement showing the arrangement of PB- $b$-PEO copolymers forming the membrane. The thickness of the hydrophobic core is about $16 \mathrm{~nm}$ and the thickness of the hydrophilic edge is $4 \mathrm{~nm}$. (b) Contact mode AFM image of the polymer membrane spread on silicon nitride surface. (c) Height profile of the line in b) showing an overall membrane thickness of $\sim 25 \mathrm{~nm}$. p. 69

Figure 3.6 (a) Fluorescence image of polymer membrane patches at the boundary between flat and porous surfaces on a silicon nitride substrate. A line profile showing the fluorescence intensity is shown below the image (b) AFM contact mode image of the same area as in $a$. Below the image, a line profile obtained from the AFM image shows the height differences between the patches. p. 70

Figure 3.7 (a) CLSM images of polymer membrane spread on porous silicon surface. (b) Contact mode AFM image of the area shown in a where the porous silicon nitride substrate is partially covered with a polymer membrane. (c) The height profile of covered and uncovered pores. p. 70

Figure 3.8 (a) Contact mode AFM image of a pore-spanning polymer membrane where individual pores were ruptured during imaging (shown with arrows) (b) enlargement of the porous area where the rupture of the membrane is visible, the arrow show the instant when the pore was uncovered. (c) CLSM image of the area imaged by AFM taken after imaging confirms that the membrane was ruptured during imaging. p. 71

Figure 3.9 Representative force indentation curves. (a) A reference indentation curve performed on bare flat silicon nitride surface. (b) Typical force indentation curve of the polymer membrane suspended over a pore. Indentation (solid line) and retraction (dotted line) do not overlap as observed in the reference curve a. p. 73

Figure 3.10 Difference between a force curve performed on the flat part of the substrate (black), force curve performed on the flat part covered with a polymer bilayer (red) and a force curve performed on bilayer-covered pore (blue). As the polymer membrane is compressed on the hard, flat surface the polymer ruptures (Rupture in the red curve). p. 74

Figure 3.11 Modified Kelvin Voigt model in series with a nonlinear spring km that represents in-plane stretching of the bilayer upon indentation. $h_{1}$ and $h_{2}$ denote the fractional indentations as a response to a stretching force. p. 77

Figure 3.12 (a) Experimental force indentation curves performed with varying velocities (500-5000 $\left.\mathrm{nm} \mathrm{s}^{-1}\right)$. (b) Modeled force indentation curves. Experiments are performed on $600 \mathrm{~nm}$ radius silicon nitride porous substrates at constant maximal indentation force of $4 \mathrm{nN}$ with a silicon nitride cantilever. p. 78

Figure 3.13 Force indentation curve ( $\left(\right.$ markers) performed at $2000 \mathrm{~nm}^{-1}$ and $2 \mathrm{nN}$ maximal indentation force was fitted with a line. p. 79 
Figure 3.14 (a) Pre-tension of the polymer membrane determined at various indentation velocities and $2 \mathrm{nN}$ maximal indentation force. (b) Elastic moduli of a polymer membrane determined at various indentation velocities and $2 \mathrm{nN}$ maximal indentation force. p. 80

Figure 3.15 (a) Experimental force indentation curves performed with varying indentation forces (2.0-6.0 $\mathrm{nN}$ ). $\mathrm{F}_{\max }$ denotes the maximal force applied during an indentation-relaxation cycle. (b) Modeled force indentation curves. Experiments are performed on $600 \mathrm{~nm}$ radius silicon nitride porous substrates at a constant velocity of $2000 \mathrm{~nm} \mathrm{~s}^{-1}$ with a silicon nitride. p. 81

Figure 3.16 Contact mode AFM images and the corresponding height profiles of (a) untreated $\mathrm{PB}_{130}-b$ $\mathrm{PEO}_{66}$ pore-spanning membrane imaged at $0.8 \mathrm{nN}$ force and (b) cross-linked $\mathrm{PB}_{130}-b-\mathrm{PEO}_{66}$ imaged at 1.4 $\mathrm{nN}$ force. p. 82

Figure 3.17 Contact mode AFM images of (a) untreated PB130-b-PEO66 pore-spanning membrane, (c) cross-linked $\mathrm{PB}_{130}-\mathrm{b}-\mathrm{PEO}_{66}$ pore-spanning membrane and (b) their corresponding height profiles. Fluorescence images of (e) untreated membrane on flat surface and (f) UV cross-linked membrane on flat surface with membrane cracks marked with an arrow. p. 83

Figure 3.18 Overlay of two force indentation curves performed on (i) cross-linked and (ii) untreated $\mathrm{PB}_{130^{-}}$ b- $\mathrm{PEO}_{66}$ pore-spanning membranes. Force indentation curves were performed at $2000 \mathrm{~nm}^{-1}$ velocity. p. 84

Figure 3.19 Force indentation curve of the cross-linked $\mathrm{PB}_{130}-\mathrm{b}-\mathrm{PEO}_{66}$ membranes ( $\circ$ markers) with a fit (solid line) obtained with Eq. 3.9. p. 85

Figure 3.20 Force indentation curves on cross-linked polymer bilayers. (a) Experimental force indentation curves performed with varying maximal indentation force (1.5-4.0 nN). (b) Modeled force indentation curves. Experiments are performed on silicon nitride porous substrates at constant velocity of $2000 \mathrm{~nm} \mathrm{~s}-1$ with a silicon nitride cantilever. p. 86

Figure 3.21 Contact mode images of a UV-treated pore-spanning polymer membrane after (a) $10 \mathrm{~s}$ of indentation at $\sim 16 \mathrm{nN}$ of force (red line), (b) $15 \mathrm{~s}$ of indentation at $48 \mathrm{nN}$ of force (black). (c) Line profiles of obtained from a and b. p. 87

Figure 3.22 Histogram representing the extent of hysteresis observed in the experimental results (black columns) in comparison to the hysteresis predicted by the modified Kelvin-Voigt model (Figure 3.11) (gray columns). The area of the hysteresis represents the loss of energy and is calculated in Joules. p. 88

Figure 3.23 Cellular membranes (a) are supported by an intricate scaffolding of the cytoskeleton leading to a laterally tensed membrane, depicted in (b). (c) A pore-spanning membrane patch mimics the tension of a cytoskeleton supported bilayer. p. 92

Figure 3.24 Contact angle measurement. To confirm the formation of a self-assembled monolayer (SAM) on gold surface, contact angle of a buffer drop deposited on (a) mercaptoethanol SAM, (b) tertadecanethiol SAM and (c) gold surface immersed in pure ethanol was measured. p. 94

Figure 3.25 (a) Chemical functionalization steps leading to modified porous Si3N4 substrates. First, $2-3 \mathrm{~nm}$ of chromium are evaporated, followed by a 10-15 nm gold layer (1-2). (b) The gold-covered surface is then oxygen and argon plasma treated and placed in a $20 \mathrm{mM}$ self-assembly solution of mercaptoethanol (3). (c) Subsequently, the functionalized substrate is placed in PBS, GUVs are immediately added and the membrane patches are formed (4). Inset: enlargement of a functionalized pore-rim showing the lipid bilayer drawn to scale. p. 95

Figure 3.26 Fluorescence images of giant unilamellar POPC vesicles labeled with $0.1 \%$ TexasRed-DHPE used to prepare pore-spanning membranes. (a) 10-15 $\mu \mathrm{m}$ diameter vesicles, (b) a vesicle inside of which smaller vesicles are enclosed and (c) $\sim 50 \mu \mathrm{m}$ giant vesicle p. 96 
Figure 3.27 (a) Time resolved fluorescence images showing the spreading of GUVs on a mercaptoethanol functionalized porous surface. (b) Schematic representation of the vesicle spreading process: i) vesicle sedimentation on the surface, ii) adhesion, iii) flattening and iv) vesicle rupture and bilayer patch formation (Scale bars: $5 \mu \mathrm{m}) \quad$ p. 97

Figure 3.28 The image was captured while focusing at the porous substrate surface. (a) vesicle has made initial contact with the surface, (b) vesicle has partially flattened on the surface, (c) although the membrane patch seems to have formed on the surface, the high intensity fluorescence edges of the "patch", the deflating area (arrow) and the increased fluorescence intensity which is much higher than the intensity of the patch beside indicate that the vesicle has deflated however it has not ruptured. (d) large pore-spanning membrane patch. p. 98

Figure 3.29 (a) asymmetric pore-spanning lipid membrane patches and (b) symmetric pore-spanning membrane patches. When present, non-continuous halo around bilayer patches are marked with an arrow. p. 99

Figure 3.30 Fluorescence image of the filopodia giving rise to excess lipid material on the pore-rims which results in increased fluorescence intensity in comparison to the membrane-spanned pores. p. 100

Figure 3.31 In (a), a confocal fluorescence laser scanning microscopy z-stack was used to obtain a 3dimensional image of a mercaptoethanol functionalized substrate where three stages of GUV spreading were captured: a sessile vesicle, pore-rim with excess lipid material and pore-spanning membrane (indicated by crossing lines). The sessile vesicle (i) located to the left of a previously ruptured vesicle (ii) exhibits a dome shape confirmed by the orthogonal cut view (top and right side panels in a). The highly fluorescent pore-rims surrounding the vesicle results from surface enhanced fluorescence originating from filopodia consisting of double-bilayers which possess fluorophores sufficiently far away from the underlying gold film. A patch of pore-spanning membrane is visible on the right side of the vesicle. The fluorescence intensity profile obtained from the line marked with an arrow shows that the fluorescence intensity originating from the lower membrane of the sessile GUV (shaded area i) is identical to that of the pore-spanning membrane (shaded area ii), which confirms that only a single bilayer spans the pores. p. 101

Figure 3.32 Fluorescence recovery after photobleaching (FRAP) was performed on a pore-spanning POPC bilayer labeled with $0.1 \mathrm{~mol} \%$ Bodipy-DHPE. (a) The fluorescence recovery of a bleached area (i) was recorded simultaneously to the reference area (ii). (b) A fit, according to the theory of Axelrod ${ }^{38}$, to the normalized fluorescence recovery curve provides a diffusion constant of $8 \pm 4 \mu \mathrm{m}^{2} \mathrm{~s}^{-1}$. (Scale bar: $5 \mu \mathrm{m}$ ) p. 102

Figure 3.33 (a) Contact mode AFM image of a bilayer membrane patch at the boundary of flat and porous area of the substrate. The membrane is visible on the flat part of the substrate. (b) An imperfection in the bilayer membrane was used to measure its thickness. The height difference of 4-5 nm corresponds to a single bilayer thickness confirming the presence of a single bilayer membrane on the substrate. p. 104

Figure 3.34 (a) Correlation between a CLSM fluorescence image of a pore-spanning membrane and (b) AFM image of the same area. (c) Cross-sections from AFM image show (i) membrane-spanned pores where 3 out of 5 pores are covered and (ii) the presence of a single bilayer membrane $(\sim 4 \mathrm{~nm}$ height difference). p. 105

Figure 3.35 (a) Fluorescence image showing membrane-covered pores and excess lipid material on the pore rims. (b) Contact mode AFM image of the same areas as shown in (a). (c) Line profile of the fluorescence intensity (dotted line) coincides with the line profile obtained from the AFM image (solid line) indicating that the increased fluorescence originates from excess lipid material (height $\sim 100 \mathrm{~nm}$ ) present on the pore-rims. p. 106

Figure 3.36 Schematic illustration showing the difference between (a) pre-stressed and (b) low tension pore-spanning bilayer lipid bilayers. p. 107 
Figure 3.37 (a) Force volume AFM image of a porous surface where only one pore is not covered with a membrane. Force indentation curves performed on a pore-rim $(\mathrm{x})$ and a pore-spanning membrane $(\bullet)$ are shown beside the image. (b) Comparison between indentation curves performed on a pore-rim ( $\mathrm{x}$ ) and an empty pore $(\square)$. In contrast to the empty pore where the tip must travel $840 \mathrm{~nm}$ before reaching a predetermined setpoint, the contact point is identical in the case of flat surface and membrane-covered pore indicating that there is no membrane invagination into the pore. p. 108

Figure 3.38 Representative force indentation curve performed on a pore-spanning POPC membrane at 2000 $\mathrm{nm} \mathrm{s}^{-1}$ velocity and $250 \mathrm{pN}$ setpoint. p. 110

Figure 3.39 Effect of indentation position away from pore center on the response of the pore-spanning membrane. (a-b) AFM images showing the position of where indentation curves were acquired, (c) force indentation curves obtained from positions shown in (a), (d) graph showing the change in the slope $\left(k_{\text {app }}\right)$ with respect to the position of the indentation. p. 111

Figure 3.40 Histograms showing the $k_{\text {app }}$ of the pore-spanning membrane patches prepared from various lipid composition GUVs where $k_{\text {app }}$ DOPC: $1.42 \pm 0.02 \mathrm{mN} \mathrm{m}^{-1}, \mathrm{k}_{\text {app }}$ POPC: $2.60 \pm 0.12 \mathrm{mN} \mathrm{m}^{-1}$ and $k_{\text {app }}$ DOPC/cholesterol (7:3): $3.82 \pm 0.16 \mathrm{mN} \mathrm{m}^{-1}$. p. 112

Figure 3.41 Comparison between two types of pore-spanning lipid membranes: 1) low tension membranes on hydrophilic $\mathrm{OH}$ terminated self-assembly monolayers on gold or 2) pre-stressed hybrid membranes on hydrophobic alkyl SAMs. (a) Force indentation curves performed on the (i) pore-rim, on a (ii) $\mathrm{DPhPC} /$ tetradecanethiol hybrid membrane (nano-BLM) and on a (iii) POPC membrane prepared according to the newly introduced protocol. (b) Enlargement of the indentation curves together with the corresponding linear regression to determine the slope $k_{\text {app }}$ : (ii) $20.4 \pm 0.2 \mathrm{mN} \mathrm{m}^{-1}$ and (iii) $1.0 \pm 0.4 \mathrm{mN} \mathrm{m}^{-1}$. p. 113

Figure 3.42 (a) AFM image where force curves were performed by choosing the pore center and (b) force volume image of the same area as shown in (a). p. 115

Figure 3.43 Variation of $k_{\text {app }}$ depending on the pore probed. p. 115

Figure 3.44 Comparison of $k_{\text {app }}$ obtained from (a) individual force indentation curves where $k_{\text {app }}: 2.22 \pm$ $0.12 \mathrm{mN} \mathrm{m}^{-1}$ and (b) from force volume images where $k_{\text {app }}: 2.1 \pm 0.2 \mathrm{mN} \mathrm{m}^{-1}$. p. 116

Figure 3.45 Typical force indentation curves obtained on (i) a pure POPC bilayer, (ii) on the same POPC bilayer in the presence of isopropanol $(0.67 \mathrm{M})$ and (iii) in the presence of $1.28 \mathrm{M}$ isopropanol. Hysteresis between the indentation (full markers) and relaxation (open markers) curves becomes more apparent with addition of isopropanol (ii versus iii)). p. 117

Figure 3.46 Histogram of $k_{\text {app }}$ shows the gradual release of tension as isopropanol is added to the POPC bilayer where $k_{\text {app }}$ POPC: $2.2 \pm 0.4 \mathrm{mN} \mathrm{m}^{-1}, k_{\text {app }}$ of POPC in $0.67 \mathrm{M}$ isopropanol: $0.9 \pm 0.3 \mathrm{mN} \mathrm{m}^{-1}$ and $k_{\text {app }}$ of POPC in 1.28 M isopropanol: $0.3 \pm 0.1 \mathrm{mN} \mathrm{m}^{-1}$. p. 118

Figure 3.47 In (a), the interaction of isopropanol with the unperturbed pore-spanning lipid bilayer is shown with some of the isopropanol molecules (green) inserting into the lipid bilayer. In (b), the insertion of isopropanol into the bilayer is facilitated by stretching and moving the bilayer in response to indentation by the AFM tip as represented by lipids with hollow head groups moving into the center of the pore. Indentation is thus proposed to increase the spacing between lipid molecules, allowing for facilitated isopropanol insertion, a process which reverses on retraction of the AFM tip. p. 119

Figure 3.48 Typical force curve acquired during a force indentation experiment on a pore-spanning membrane showing an indentation of the membrane $\left(k_{\text {app }}\right.$ of $\left.0.001 \mathrm{~N} \mathrm{~m}^{-1}\right)$ and a plateau (approx. $50 \mathrm{pN}$ in height) p. 120 
Figure 3.49 Schematic representation of an indentation experiment where (a) AFM tip is far away from the pore-spanning membrane, (b) AFM tip is indenting the membrane, red circles indicate areas where membrane experiences slight degree bending (c) a tether is pulled out as of the membrane as the AFM tip is retracted, circle indicated area where membrane experiences high degree of bending.

p. 121 


\section{LIST OF TABLES}

Table 1.3 Structure and transition temperatures of common membrane phospholipids $\quad$ p.4

Table 1.4 Phospholipid, cholesterol and glycolipid composition of various plasma membranes. p.8

Table 3.1 Summary of viscoelastic properties of native polymer and UV cross-linked membranes. p.89 


\section{INTRODUCTION}

\subsection{MEMBRANES}

Defined as the single smallest unit of life, a cell is a complex assembly of organelles essential for the cellular replication, energy production and growth. The different cellular components bathe in a cellular matrix enclosed in an intricate scaffolding of cytoskeleton which is enveloped in cellular membrane. This membrane mainly composed of phospholipids and proteins delimits the cellular boundary and isolating the cell contents from the outer medium.

\subsubsection{FUNCTIONS IN LIVING ORGANISMS}

Despite their remarkably thin structure $(\sim 5 \mathrm{~nm})$, cellular membranes perform many vital cellular functions. Its primary role is to define the cell boundaries by giving it its shape and size. Protists, fungi, plants as well as animals are composed of eukaryotic cells whose size varies between $10 \mu \mathrm{m}$ and $30 \mu \mathrm{m}$ for most animal cells and between $10 \mu \mathrm{m}$ and 100 $\mu \mathrm{m}$ for plants. ${ }^{1}$ Beyond size definition, cellular membranes allow for compartmentalization (Figure 1.1a). Internal volume division in organelles allows for distinct unrelated processes to take place simultaneously within the same cell. Furthermore, cellular membranes act as an organization medium for biochemical activities by accommodating various proteins (integral or peripheral) which are part of cellular machinery. An example of a crucial process performed at the interface of the inner mitochondria membrane is the energy conversion process (oxidative phosphorylation) (Figure 1.1b). Cellular membranes also function as selectively permeable barriers allowing for uptake and excretion of compounds by means of channels and pumps imbedded in the membrane. It is the control of ion flow into and out of the cell which generates chemical and electrical signals responsible for biological communication such as action potentials in nerve cells (Figure 1.1c). Additionally, carbohydrates attached to lipids allow the cellular membrane to detect external signals and therefore allows for intercellular recognition (Figure $1.1 \mathrm{~d}$ ). ${ }^{2}$ 
a)

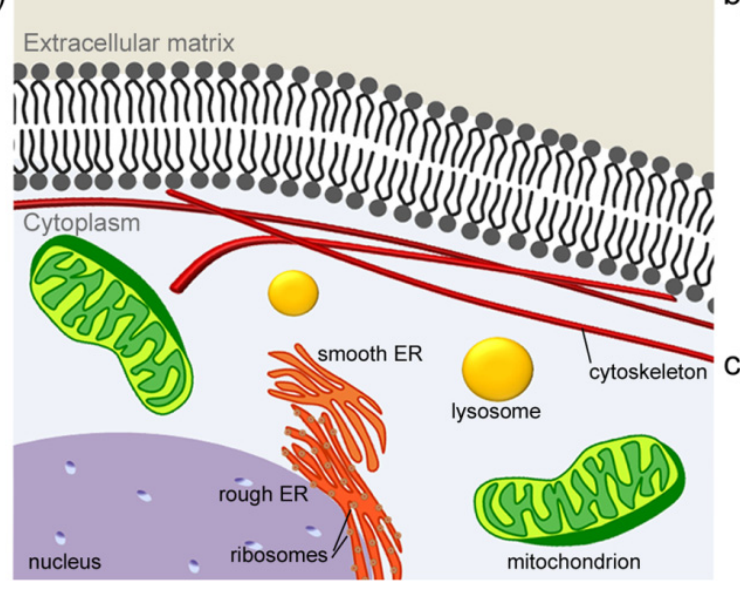

b) Mitochondrion intermembrane space

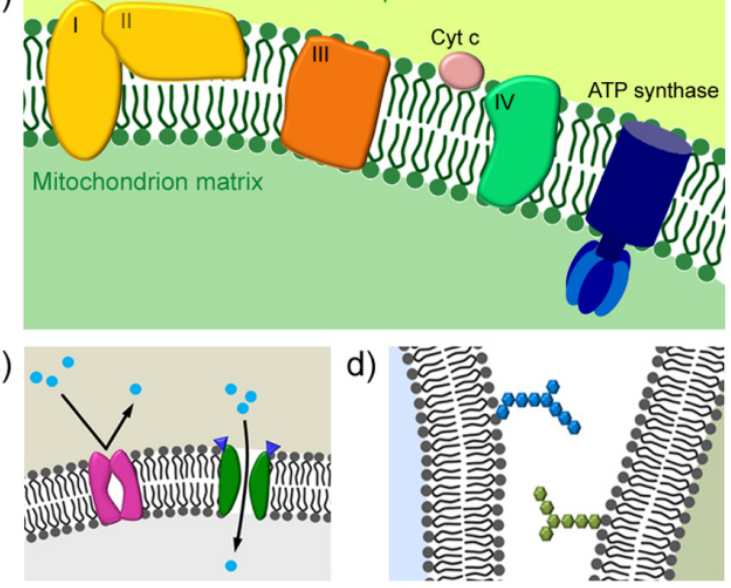

Figure 1.1 Schematic representation of membrane functions. In (a), membrane acts as a boundary between the extracellular matrix and the cytoplasm. Organelles are compartmentalization examples. (b) Oxidative phosphorylation in the mitochondrion where the membrane acts as a scaffold for chemical activity. (c) Channels embedded in the membrane allow for membrane transport and action potential generation. (d) Intercellular recognition through glycolipids present in the cellular membrane.

The cell membrane not only acts as a boundary between cell interior and its environment but it is also an active component of the cell's biological and biochemical activities. Better understanding of the properties of the cellular membrane might also elucidate the functioning of the many processes associated with it.

\subsubsection{MEMBRANE COMPOSITION AND PROPERTIES}

The different functions performed by the cellular membrane are related to the membrane lipid composition, which plays a pivotal role in defining its structural, chemical and mechanical properties. Defined as two-dimensional liquids, cellular membranes are composed mainly of lipids and proteins. It is the specific assembly of these components that confers the membrane its characteristics. The ratio of lipid to protein varies depending on the type of cellular membrane (i.e. plasma membrane versus inner mitochondrion membrane), the type of organism (i.e. plant versus animal) and the type of cell (i.e. muscle versus liver). ${ }^{1}$ Nevertheless, there are approx $5 \times 10^{6}$ lipid molecules in $1 \mu \mathrm{m} \times 1 \mu \mathrm{m}$ area of lipid bilayer or about $10^{9}$ lipid molecules in the plasma membrane of a small animal cell. ${ }^{2}$ 
a)

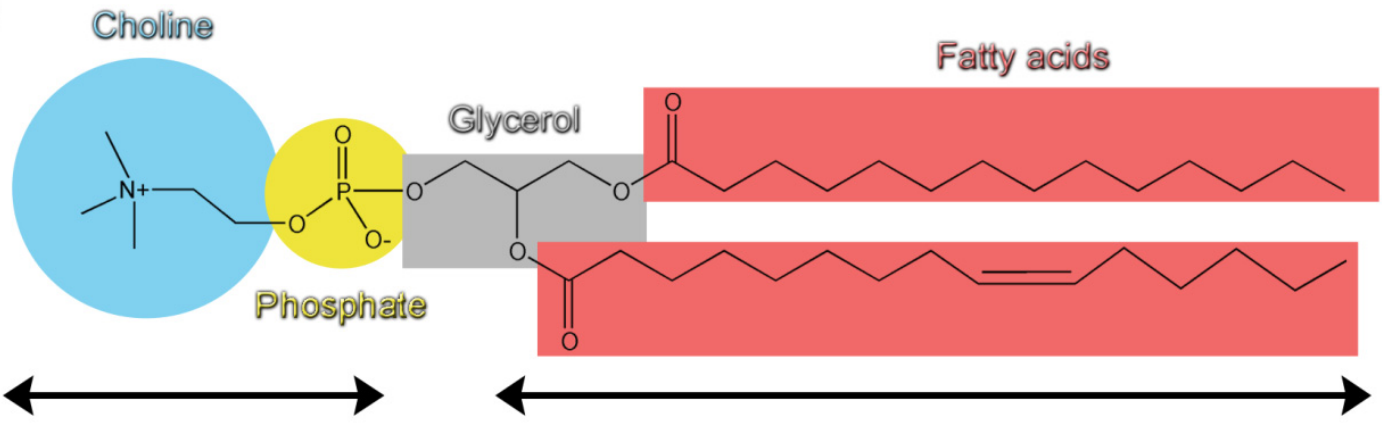

Hydrophilic head

Hydrophobic tails

b)

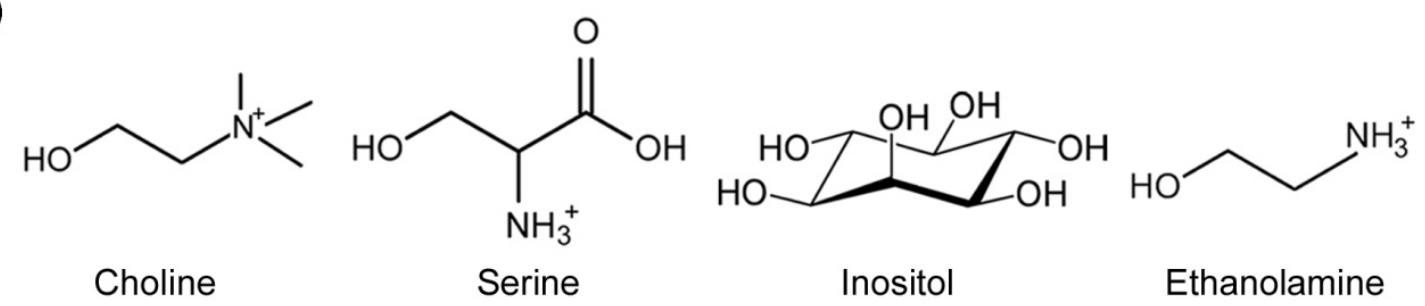

Figure 1.2 (a) Chemical structure of a phospholipid with a schematic representation of the phospholipid hydrophilic head and hydrophobic tails. (b) Chemical structures of the most common alcohol head groups of phospholipids.

Phospholipids, glycolipids and cholesterol are the three major kinds of lipids that are encountered in cellular membranes. Phospholipids (Figure 1.2a) derived from glycerol are called phosphoglycerides and consist of a glycerol backbone, two fatty acid chains and a phosphorylated alcohol. The most common alcohol moieties of phosphoglycerides are: serine, ethanolamine, choline, glycerol and inositol (Figure 1.2b). The size and charge of these alcohols have an impact on the packing of the phospholipids, which in turn affects the final membrane curvature. The length of the fatty acids range between 14 and 24 carbons with 16 and 18 carbons being the most common in animal membranes. ${ }^{3}$ Both the length and the degree of saturation of the fatty acid chains determine the phospholipids phase transition temperature, which is a temperature at which the lipids undergo transition from gel phase (solid) to liquid phase (Table 1.1). In the liquid phase, the lipids can diffuse freely in the $2 \mathrm{D}$ plane. 
Table 1.1 Structures and transition temperatures of common membrane phospholipids.

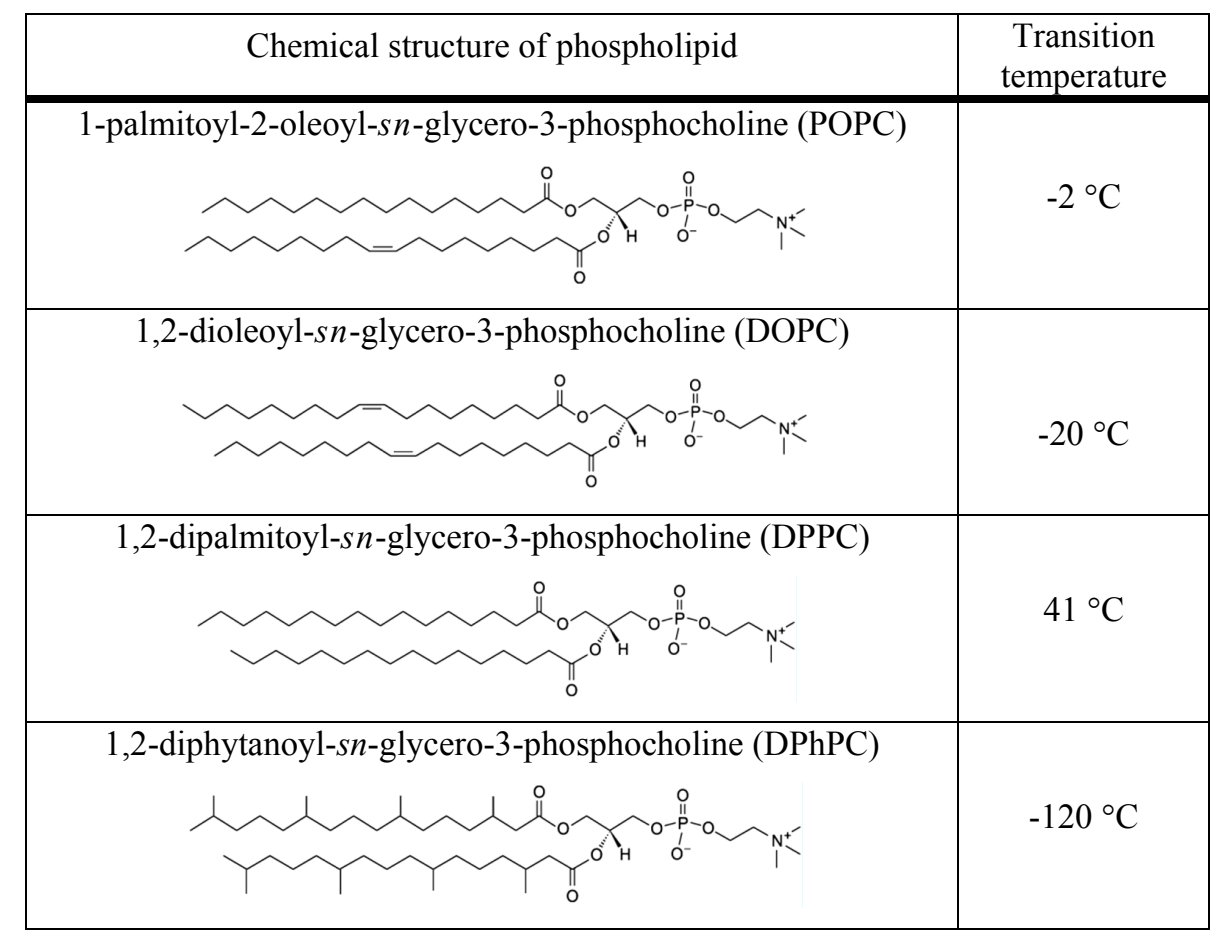

In phospholipids, the fatty acid chains are called "tails" and the phosphorylated alcohol group is called "head". It is the particular structure of phospholipids that confers them an amphiphilic character. Amphiphile is a chemical term given to compounds which posses both: hydrophobic and hydrophilic properties. The hydrophobic tail is a hydrocarbon moiety, which varies in length (number of carbons) and degree of saturation. The hydrophilic part is a charged group (carboxylate, sulfate, amine, phosphate or other) or a polar uncharged group.
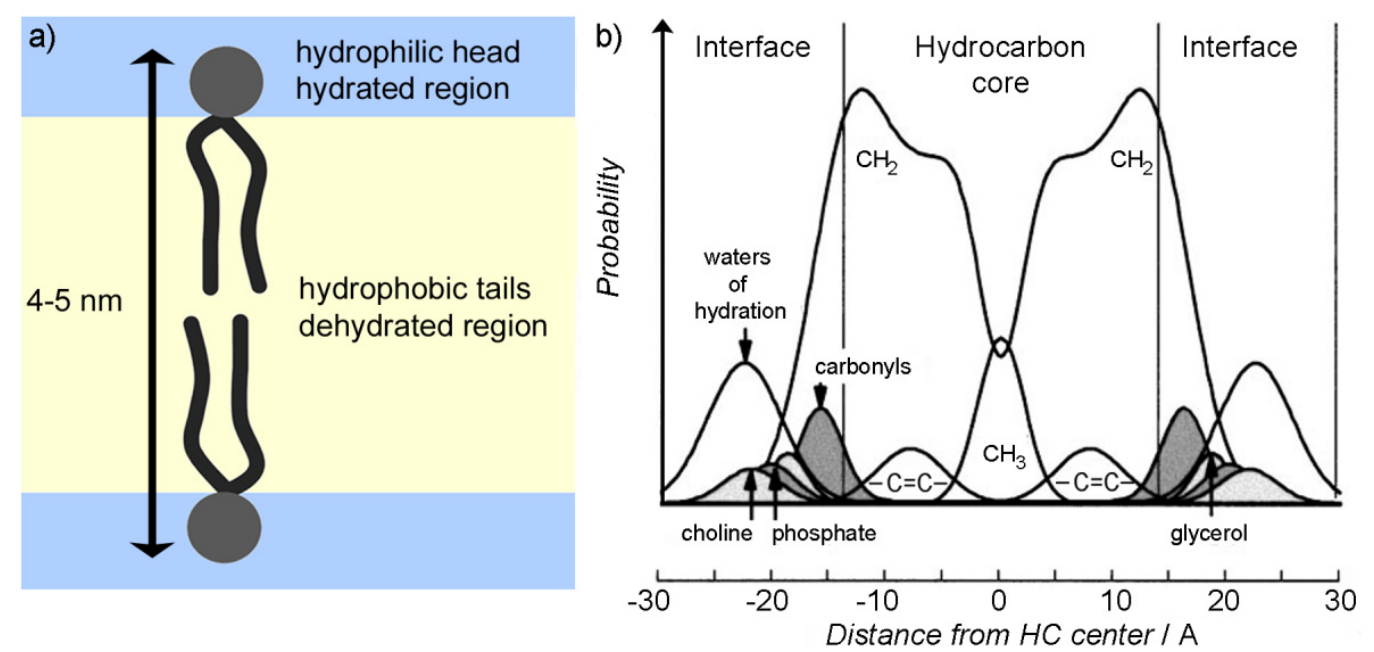

Figure 1.3 (a) Schematic representation of phospholipid assembly to form a bilayer structures with hydrophobic tails forming the membrane core and the heads interacting with the aqueous environment. (b) A profile obtained from X-ray and neurton diffraction data showing the distribution of structural groups in a DOPC bilayer as a function of distance from the hydrocarbon core (HC). Figure taken from White et al. ${ }^{4}$ 
The hydrophobic effect is a phenomenon observed when non-polar molecules are in contact with a polar environment such as water. In bulk, each water molecule takes part, on average, in 3-3.5 hydrogen bonds (H-bonds) with surrounding water molecules. ${ }^{5}$ When a non-polar molecule is introduced into a water environment, water molecules either lose H-bonds between each other or rearrange themselves around the non-polar molecules so that no H-bonds are lost. Even through rearrangement of water molecules preserves all of the initial H-bonds, the rearrangement itself results in a more ordered structure of water molecules which is entropically unfavorable in comparison to bulk water. In order to diminish the loss of entropy, non-polar molecules aggregate together so that the surface in contact with water is minimized (i.e. loss of H-bonds of water with itself is kept to a minimum). This clustering results in segregation of water molecules and non-polar molecules and is known as hydrophobic effect. It is due to the hydrophobic effect that when amphiphiles are placed in aqueous solution, various aggregates such as micelles, vesicles or bilayers, are spontaneously formed by self-assembly. By aggregating, the area that the water molecules must solvate is decreased which in turn maximizes the entropy of water (in contrast to decrease of entropy when ordered network of water dipoles are formed).

Shape of lipid molecules affects their packing upon clustering, which in turns determines the shape of the aggregates formed. Israelachvili proposed that packing properties of an amphiphile are related to the optimal area $a_{0}$, the volume $V$ of the hydrocarbon tails and the maximum effective length of the tails $l_{c}$. A dimensionless packing parameter $\mathrm{P}$ defined as $\frac{V}{a_{0} l_{\mathrm{c}}}$, is used to determine the type of the aggregate formed: spherical micelle $\left(\frac{V}{a_{0} l_{\mathrm{c}}}<1 / 3\right)$, non spherical micelles $\left(1 / 3<\frac{V}{a_{0} l_{\mathrm{c}}}<1 / 2\right)$, vesicles or bilayers $\left(1 / 2<\frac{V}{a_{0} l_{\mathrm{c}}}<1\right)$ or inverted structures $\left(\frac{V}{a_{0} l_{\mathrm{c}}}>1\right)$ (Figure 1.4). 


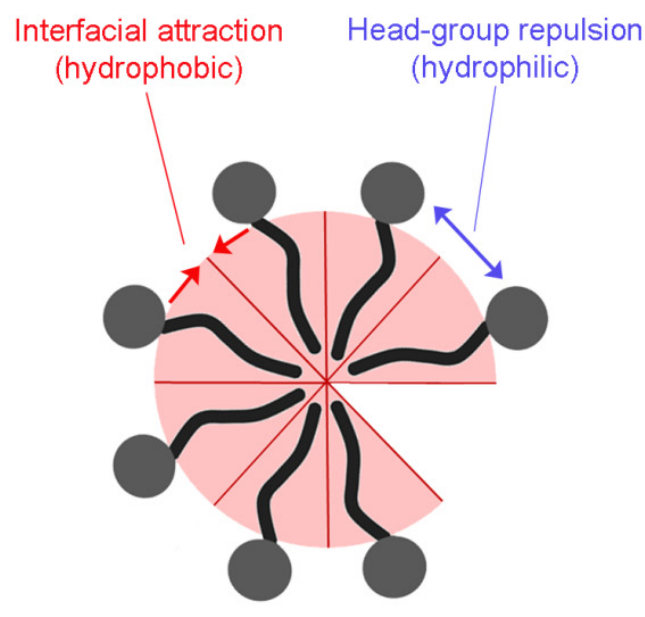

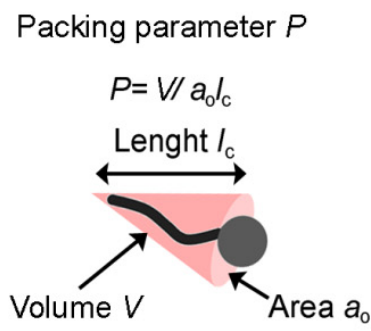

Cone and truncated cone (spherical, rodlike micelles)

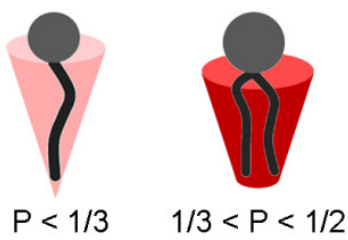

Truncated cone and cylinder (vesicles and bilayers)
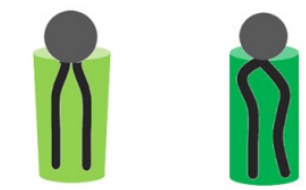

$1 / 2<\mathrm{P}<1 \quad \mathrm{P}=1$

Reverse truncated cone (reverse micelles)

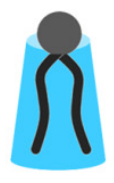

$\mathrm{P}>1$

Figure 1.4 Packing parameter $P$ which is the ratio of the volume of the hydrophobic chain $V$ to the area $a_{\mathrm{o}}$ and the maximal length of the hydrophobic chain $l_{c}$ and predicts the structure of the aggregates formed from amphiphiles placed in aqueous solution.

Spherical micelles are usually formed from surfactant molecules such as sodium palmitate which have a single hydrophobic chain attached to the head. Micelle size is usually limited to about $20 \mathrm{~nm}$. Micelles are not favored geometrical arrangements for phospholipids because of the bulkiness of two fatty acid chains. In contrast, phospholipids readily form bimolecular sheets (bilayers) which can be macroscopic in dimensions. The formation of bilayers in aqueous environment is driven by the hydrophobic effect. Hydrophobic fatty acid tails are shielded from water molecules inside the bilayer and the hydrophilic head groups interact with the water, hence maximizing the entropy of the system. The formation of lipid bilayers is a self-assembly process where van der Waals attractive forces and hydrophobic interactions between the phospholipid tails favor the close packing of the phospholipid molecules. Furthermore, electrostatic and hydrogen bonding interactions between the polar head groups of the phospholipids and the water molecules further stabilize the bilayer structure and the steric head group repulsion. In general, a phospholipid bilayer thickness is $4-5 \mathrm{~nm}$ and the cross sectional area of a single phospholipid chain is $0.20 \mathrm{~nm}^{2}$. The average surface area of the bilayer occupied by a single phospholipid lipid is $0.4-0.7 \mathrm{~nm}^{2}{ }^{6}$ Curvature and fluidity of the bilayer depend on the length of the phospholipid tails, the size of the phospholipid heads, the ratios of different lipids composing the bilayer and the amount of cholesterol present in the membrane. Since the forces acting between the phospholipid molecules are non covalent, the molecules can rotate in the bilayer and exchange positions with their neighbors $\left(\sim 10^{7}\right.$ 
times per second) giving rise to phospholipid lateral diffusion with diffusion coefficients of about $1 \cdot 10^{-8} \mathrm{~cm}^{2} \mathrm{sec}^{-1}$. Phospholipid flip-flops, where phospholipids in opposing leaflets swap positions, are observed but are rare (approx. once every $\sim 1 \cdot 10^{5} \mathrm{~s}$ ). ${ }^{2}$ Membrane fluidity is controlled by the length and degree of saturation of the phospholipid tails. Longer fatty acid chains with unsaturated acyl groups result in more disordered and fluid bilayers. In addition to the phospholipid chemical structure, the presence of cholesterol also affects the membrane fluidity. Cholesterol is a steroid present in animal plasma membranes where its amount varies between $20-40 \%$ by total lipid weight and influences membrane stiffness and tension. ${ }^{7}$ Cholesterol hydroxyl group interacts with head group region of the phospholipid while the rest of the molecule aligns within the fatty acid region of the phospholipid (Figure 1.5a). Cholesterol affects membrane fluidity by sterically blocking large motions of the fatty acid tails which decreases membrane fluidity.

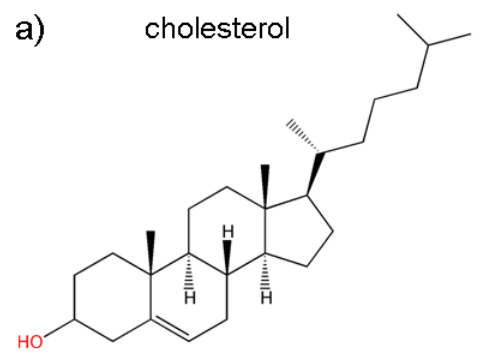

b)

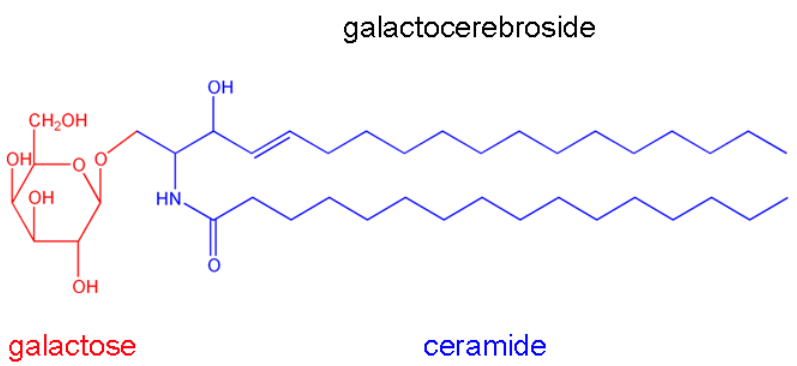

Figure 1.5 Chemical structures of (a) cholesterol and (b) galactocerebroside, the simplest glycolipid with a galactose sugar residue.

Glycolipids are carbohydrate containing lipids. Cerebroside is the simplest glycolipid and has only one sugar residue, either glucose or galactose (Figure 1.5b). The carbohydrate content of eukaryotic plasma membranes is approximately 2-10\% by weight in the form of glycolipids or glycoproteins. ${ }^{1}$ Glycolipids are oriented in the membrane so that the sugar residues are always on the extracellular side and are the basis of cellular recognition. Approximate amounts of phospholipids, cholesterol and glycolipids found in plasma membranes depend on the cell type and are shown in Table 1.2. 
Table 1.2 Phospholipid, cholesterol and glycolipid composition of various plasma membranes ${ }^{2}$

\begin{tabular}{|c|c|c|c|c|c|c|}
\hline Lipid & $\begin{array}{l}\text { Liver cell } \\
\text { plasma } \\
\text { membrane }\end{array}$ & $\begin{array}{l}\text { Red blood } \\
\text { cell plasma } \\
\text { membrane }\end{array}$ & Myelin & Mitochondrion & $\begin{array}{l}\text { Endoplasmic } \\
\text { reticulum }\end{array}$ & $\begin{array}{c}\text { E.coli } \\
\text { bacterium }\end{array}$ \\
\hline Cholesterol & 17 & 23 & 22 & 3 & 6 & 0 \\
\hline $\begin{array}{l}\text { Phosphatidyl- } \\
\text { ethanolamine }\end{array}$ & 7 & 18 & 15 & 25 & 17 & 70 \\
\hline $\begin{array}{l}\text { Phosphatidyl- } \\
\text { serine }\end{array}$ & 4 & 7 & 9 & 2 & 5 & trace \\
\hline $\begin{array}{l}\text { Phosphatidyl- } \\
\text { choline }\end{array}$ & 24 & 17 & 10 & 39 & 40 & 0 \\
\hline Sphingomylein & 19 & 18 & 8 & 0 & 5 & 0 \\
\hline Glycolipids & 7 & 3 & 28 & trace & trace & 0 \\
\hline Others & 22 & 13 & 8 & 21 & 27 & 30 \\
\hline
\end{tabular}

Phospholipids and glycolipids confer the membrane its structural and biochemical properties, but it is the role of proteins associated with the membrane to mediate the distinctive membrane functions such as transport, communication and energy transduction. Membranes proteins which span the entire thickness of the bilayer are called transmembrane proteins (Figure 1.6a), whereas those which attach either to another protein or interact with one leaflet of the bilayer are referred to as peripheral proteins (Figure 1.6b).

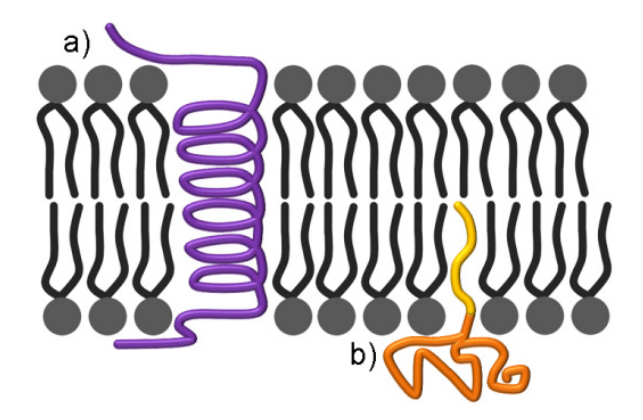

Figure 1.6 Schematic representation of (a) transmembrane protein and (b) peripheral membrane protein

The fluid mosaic model introduced in 1972 by Singer and Nicolson, was the first widely accepted model of the general organization of the plasma membrane. ${ }^{8}$ This model proposed a cellular membrane organization where globular proteins alternate in a phospholipid bilayer. Based on numerous experimental observations, this description of the cellular membrane was the only one which agreed with the experimental findings at that time. The most important features of the fluid mosaic model were: the hydrophobic and hydrophilic interactions between the molecules composing the membrane (i.e. 
phospholipids), the presence of different types of membrane components (peripheral and integral proteins) and the overall fluid nature of the membrane in the 2-dimensional plane. With the advance of membrane research and the inability of this model to explain the experimentally observed results, it became clear that the fluid mosaic model oversimplified the complexity of the plasma membrane. Refined models of the plasma membrane organization began surfacing.

Membrane skeleton fence model of the plasma membrane proposed by Kusumi, Sako and Yamamoto describes the fact that the experimentally observed long-time diffusion rates of proteins in the plasma membrane of live cells were slower than those predicted, and that the diffusion was limited to finite-sized domains. ${ }^{9}$ The fence model explained these findings by the interaction of the plasma membrane with the cytoskeleton, a meshwork composed of actin filaments and actin-binding proteins. According to this model, the diffusive properties of the proteins are influenced by the steric interactions of the cytoplasmic domains of transmembrane proteins with the cytoskeleton. ${ }^{10}{ }^{11}$ The model was named fence model because the actin strands in the cytoskeleton network act as fences to confine the transmembrane proteins to defined areas.

In the anchored-protein picket model, the cytoskeleton indirectly interacts with the phospholipids in the outer membrane leaflet. The pickets (i.e. cytoskeleton-anchored proteins) slow down phospholipid diffusion through increased packing around the proteins or through increased hydrodynamic friction. ${ }^{11-13}$

Simons and Ikonen have proposed a different model, based on the raft hypothesis. ${ }^{14}$ Rafts are defined as dynamic, nanoscale $(<100 \mathrm{~nm})$, sterol-sphingolipid-enriched, ordered assemblies of proteins and lipids. Rafts in plasma membranes are essentially microdomains that could selectively include and exclude proteins. ${ }^{15-17}$ However, much skepticism still exists with respect to the raft hypothesis due to the difficulty of obtaining direct experimental evidence of the existence of lipid rafts. ${ }^{18,19}$

\subsubsection{MEMBRANE PHYSICS}

Many membrane processes depend on the fact that the membrane can undergo elastic (i.e. reversible) deformations. The elastic behavior of the membrane, when undergoing 
mechanical stresses, is governed by its stretching elasticity, bending rigidity and lateral pre-tension. These mechanical properties of the membrane are closely associated with the chemical nature of the membrane constituents and the forces acting between them.

\subsubsection{BILAYER STRETCHING}

Bilayer membrane can be considered as a homogenous rigid thin square plate with a thickness $d_{\mathrm{p}}$. Stretching it can be defined as application of stress tensor $\sigma_{\mathrm{xx}}=\sigma_{\mathrm{yy}}=\mathrm{S}$ resulting in a strain tensor $u_{\mathrm{xx}}=u_{\mathrm{yy}}=\mathrm{S}\left(2 / 9 K_{\mathrm{v}}+1 / 6 \mu\right)$ where $K_{\mathrm{v}}$ is the volume compression modulus of the plate and $\mu$ is the shear modulus. Practically, the strain is translated into a relative area change $u_{\mathrm{xx}}+u_{\mathrm{yy}}$ according to $\tau=K_{\mathrm{A}}\left(u_{x x}+u_{y y}\right)$ where $\tau$ is the applied lateral stress (tension) and $\tau=\mathrm{S} d_{\mathrm{p}}$ and $K_{\mathrm{A}}$ is the area compression modulus. After substitution and rearrangement,

$$
K_{\mathrm{A}}=\frac{d_{p} K_{v}}{\left(\frac{4}{9}+\frac{K_{v}}{3 \mu}\right)}
$$

Since $K_{\mathrm{v}} \sim 3 \mu$ for many materials, $K_{\mathrm{A}}$ linearly depends on the plate thickness $d_{\mathrm{p}}$. However, experimental results do not support this relationship. Therefore, instead of depicting the membrane as a rigid plate, membrane stretching can be considered in terms of amphiphile interactions at the amphiphile-water interface in response to stress. When a monolayer forms at the water-air interface, the mean interface area $a$ occupied by the amphiphile is defined from the compromise between the steric repulsion between the amphiphiles and the surface tension.

When the amphiphiles are densely packed, the hydrocarbon chain are shielded from water, however the repulsive energy between the amphiphile heads rises according to $1 / a$. When the density of amphiphiles is low, the repulsion term is inexistant; however, the exposure of the hydrocarbon chains increases the energy according to $\gamma a$ where $\gamma$ is the surface tension. 


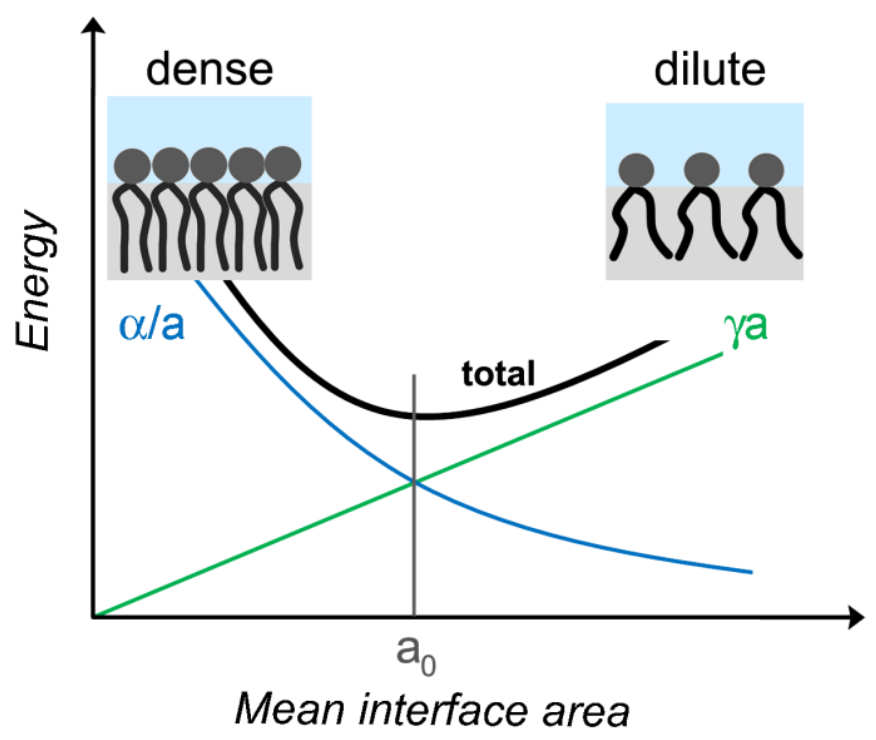

Figure 1.7 Relationship between the energy and the mean interface area $a$ of an amphiphile at the water air interface. At high density, the energy increase with increasing packing of phospholipids; at low density, the energy increases with exposure of hydrocarbon tails. $a_{0}$ is the optimal interface area of an amphiphile.

The energy $E_{\mathrm{A}}$ associated with the average area occupied by an amphiphile is given by:

$E_{\mathrm{A}}=\frac{\alpha}{a}+\gamma a$

where $\alpha$ is a constant. ${ }^{5}$

Considering that the energy can be minimized when $a=a_{0}$, and by setting $d E_{\mathrm{A}} / d a=0$, $a_{0}$ can be obtained from $a_{0}=\sqrt{\left(\frac{\alpha}{\gamma}\right)}$. Subsequently, eq 1.2 can be rewritten:

$$
E_{\mathrm{A}}=2 \gamma a_{0}+\left(\frac{\gamma}{a}\right)\left(a-a_{0}\right)^{2}
$$

When $a$ moves away from $a_{0}$, only the second part of the energy equation is affected. Near equilibrium, where $a=a_{0}$, the energy of an amphiphile changes by $\left(\frac{\gamma}{a}\right)\left(a-a_{0}\right)^{2}$ and when divided by area per amphiphile $a_{0}$ it becomes $\gamma\left[\left(a-a_{0}\right) / a_{0}\right]^{2}$. This part of the equation can be rewritten as $\left(\frac{K_{\mathrm{A}}}{2}\right)\left(u_{x x}+u_{y y}\right)^{2}$ where $u_{x x}+u_{y y}=$ relative change in 
area $=\left(a-a_{0}\right) / a_{0}$. Finally, a relationship between surface tension and area compression modulus $K_{\mathrm{A}}$ is:

$K_{\mathrm{A}}=2 \gamma$ (for a monolayer)

Eq. 1.4

$K_{\mathrm{A}}=4 \gamma$ (for a bilayer)

Eq. 1.5

Accordingly to this relationship, $K_{\mathrm{A}}$ of a lipid bilayer should be around $0.008-0.2 \mathrm{~J} / \mathrm{m}^{2}$ and be independent of bilayer thickness. Experimentally, stretching modulus of membranes can be measured by performing micropipette aspiration experiments. Rawicz et al. performed experiments on fluid phase diacyl phospatidylcholine bilayers with varying length (13 to 22 carbons) and determined $K_{\mathrm{A}}$ values between $230 \mathrm{mN} \mathrm{m}^{-1}$ and $250 \mathrm{mN} \mathrm{m}^{-1}\left(0.230\right.$ and $\left.0.250 \mathrm{~J} / \mathrm{m}^{2}\right){ }^{20}$

\subsubsection{BILAYER BENDING}

Bending of a bilayer membrane requires energy due to the resistance of amphiphiles to undergo rearrangement which brings them away from their equilibrium positions. During bending, phospholipid molecules in the upper bilayer leaflet are pulled apart while the phospholipids in the bottom leaflet are compressed (Figure 1.8). The resistance towards bending is characterized by bilayer bending rigidity $\kappa_{\mathrm{b}}$.

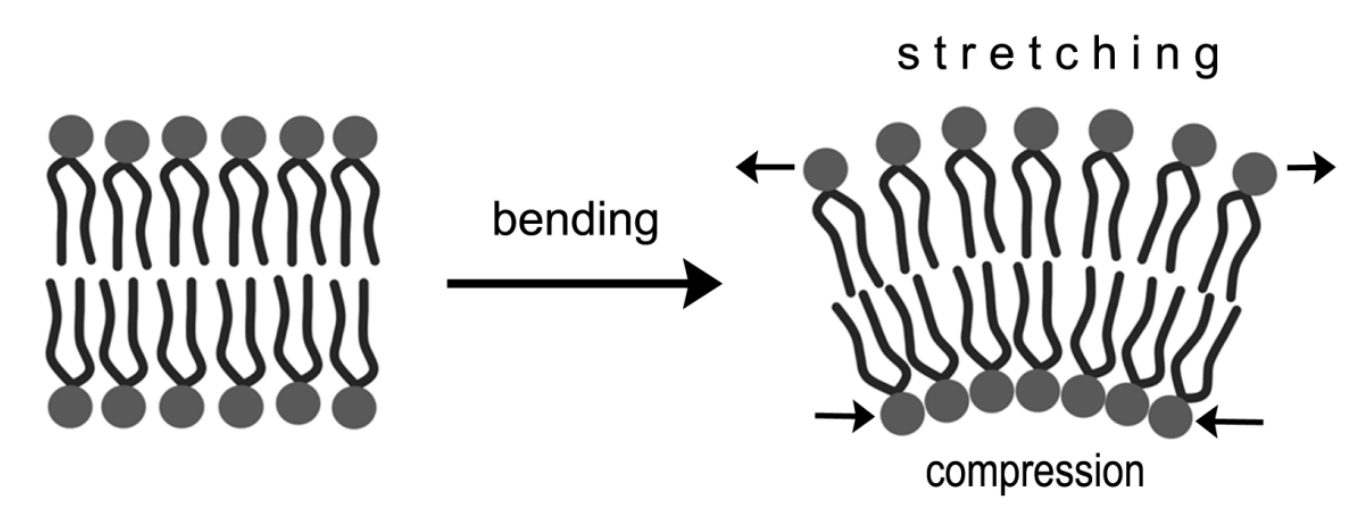

Figure 1.8 During bilayer bending, the amphiphiles in the upper leaflet are pulled apart while the amphiphiles in the bottom leaflet are compressed.

Bending deformation of the membrane alters the area per amphiphile based on the bending rigidity $\kappa_{\mathrm{b}}$ and Gaussian rigidity $\kappa_{\mathrm{G}}$ according to: 
$E=\left(\frac{\kappa_{\mathrm{b}}}{2}\right)\left(\frac{1}{R_{1}+R_{2}}\right)^{2}+\frac{\kappa_{\mathrm{G}}}{R_{1}+R_{2}}$

where $\mathrm{R}_{1}$ and $\mathrm{R}_{2}$ are two principal radii of curvature.

The magnitude of the strain associated with the stretching and compression of the bilayer leaflets during bending are governed by area compression modulus $K_{\mathrm{A}}$. Bending rigidity can therefore be calculated using $K_{\mathrm{A}}$ following:

$$
\kappa_{\mathrm{b}}=\frac{K_{\mathrm{A}} d_{\mathrm{bl}}^{2}}{\alpha}
$$

where $\alpha=12,24$ or 48 for uniform rigid plate, for polymer brush and for two leaflets free to slide past each other, respectively.

It has been experimentally observed that when not constrained, a bilayer membrane undulates at room temperature. By measuring the tension required to flatten out these thermal undulations and the subsequent increase in area of the bilayer, apparent area compression modulus can be obtained following:

$$
K_{\mathrm{A}, \text { app }}=\frac{K_{\mathrm{A}}}{\left[\frac{1+K_{\mathrm{A}} k_{\mathrm{B}} T}{\left(8 \pi \kappa_{B} \tau\right)}\right]}
$$

where $\tau$ is the applied tension. At low tensions, $K_{\mathrm{A} \text {, app }} \sim \frac{8 \pi \kappa_{B} \tau}{k_{\mathrm{B}} T}$ and it is only at high tensions that $K_{\mathrm{A} \text {, app }} \sim K_{\mathrm{A}}$. Bending rigidity $\kappa_{\mathrm{b}}$ can be extracted from $K_{\mathrm{A} \text {, app calculated }}$ from an experimentally obtained stress vs. strain curves at small stress. Typical values for $\kappa_{\mathrm{b}}$ found for lipid bilayer range between $0.2 \times 10^{-19}$ and $1.0 \times 10^{-19} \mathrm{~J}$ which corresponds to $5-25 \mathrm{k}_{\mathrm{B}} \mathrm{T}^{6}$

\subsubsection{MEMBRANE TENSION}

Membrane deformation processes, which include endocytosis, exocytosis, cell motility and spreading, as well as membrane trafficking and repair are processes regulated by 
cellular membrane tension. ${ }^{21-28}$ The main contribution to the overall tension in the plasma membrane originates from its adhesion to the underlying actin cytoskeleton, while the inherent tension of the lipid bilayer itself is at least an order of magnitude smaller and therefore negligible. On a mesoscopic scale, native lipid bilayers connected to the cytoskeleton have been shown to exhibit a moderate lateral tension $\left(\sigma=10^{-2}-10^{-1} \mathrm{mN} \mathrm{m}^{-}\right.$ $\left.{ }^{1}\right){ }^{26,29}$ 


\subsection{MOdEL MEMBRANE SYSTEMS}

Native cellular membranes are composed of thousands of interrelated components and the study of individual processes is therefore hindered by a large amount of uncontrollable variables. To circumvent the complexity of native systems, the use of model systems, where components can be incorporated and modified step-wise, is a rational basis to systematically investigate the factors affecting the function of biological systems. Model systems are designed with the objective to mimic the properties of native systems, while significantly decreasing their complexity. In the case of cellular membranes, model membrane systems should display the characteristics of a native cell membrane such as membrane fluidity, similar membrane tension and robustness. It is also desirable for model membranes to be relatively simple to prepare, reproducible and to have control over lipid membrane composition. Furthermore, it should be possible to study the model membrane by various analytical techniques in order to obtain reproducible results. Various types of model membranes have been developed to study intrinsic membrane properties (i.e. membrane mechanics, lipid diffusion constants) ${ }^{30-35}$ as well as the properties of membrane components (i.e. activity of membrane channels). ${ }^{36-39}$

\subsubsection{LIPOSOMES}

Liposomes are vesicles composed of phospholipids where the aqueous interior is separated from the outside environment by a bilayer of amphiphilic molecules. ${ }^{40}$ The exact mechanism of GUV formation is not known, however it has been observed that, in air, phospholipids self-assemble to form bilayer sheets. Upon addition of water, the hydrophilic head groups of phospholipids are hydrated. Then, as water penetrates between the bilayer sheets (swelling of bilayers), bilayers separate, bend and spontaneously curve to form vesicles. ${ }^{41-43}$

Vesicles of different sizes are named accordingly; multilamellar vesicles (MLV), small unilamellar vesicles (SUV), large unilamellar vesicles (LUV), and giant unilamellar vesicles (GUV). ${ }^{43,44}$ Although MLVs are often quite large in size (usually larger than 1 $\mu \mathrm{m})$, their name is associated with their onion-like structure, consisting of multiple layers instead of their size. The limited experimental control over their structure limits their applicability as a model system. 
There are several methods commonly used prepare liposomes in the laboratory. In general, the first step consists of phospholipid films preparation on flat surfaces. Films are obtained by depositing phospholipids, dissolved in chloroform, on a flat surface such as glass. The surface is heated so that the solvent evaporates and the phospholipids rearrange into ordered layers, this step can be done under vacuum to speed up the process.
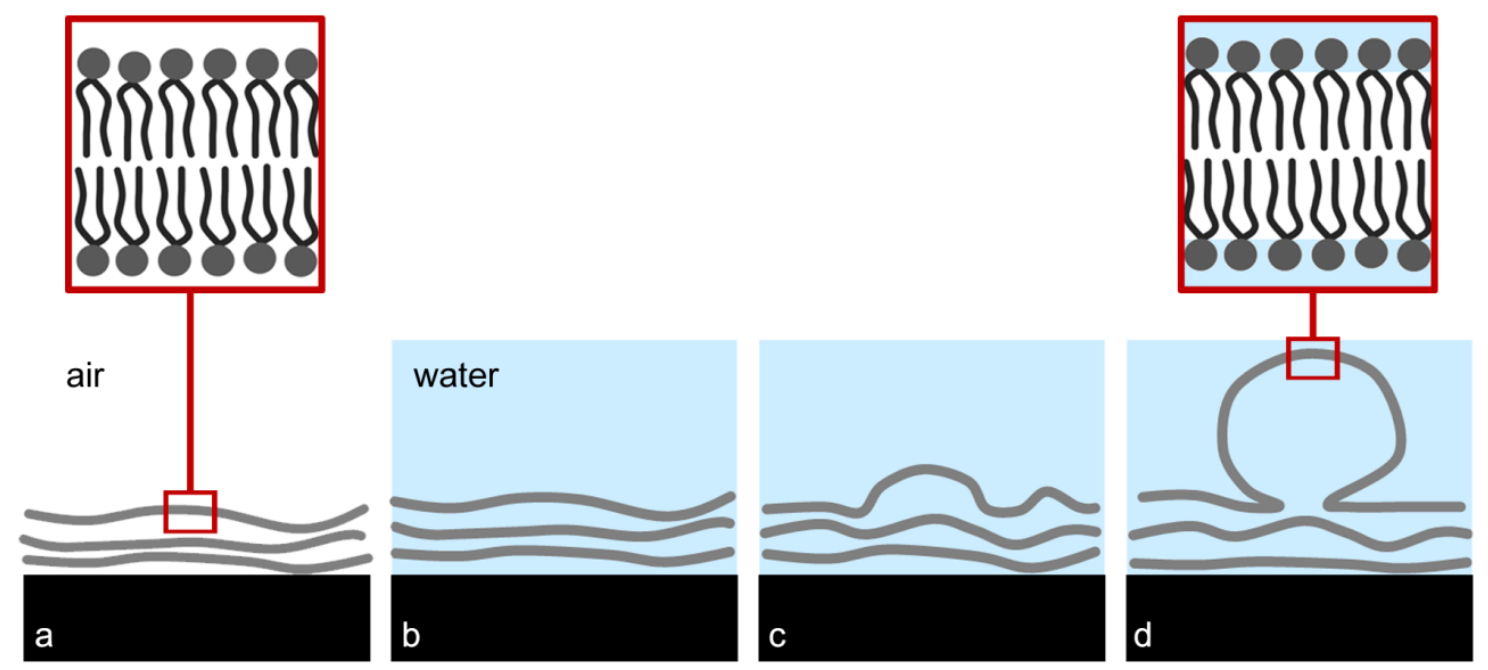

Figure 1.9 Formation of liposomes from flat phospholipid bilayer films. The films are hydrated and the liposomes are created by swelling and closing of bilayer on themselves (adapted from Walde et al. ${ }^{43}$ ).

Following film formation, aqueous solution is added and as the phospholipid films are hydrated. As the space between the films increases, the bilayer edges join, creating vesicles (Figure 1.9). Various approaches are used to produce different size vesicles. To form SUVs, which are smaller than $50 \mathrm{~nm}$ in diameter, sonication of lipid films in aqueous solution is performed. To produce LUVs, extrusion is used where the lipid film solution is repeatedly forced through a size-selective polycarbonate membrane filters with pore sizes ranging from 50 to $5000 \mathrm{~nm}$. LUVs, which range in size between 50 and $1 \mu \mathrm{m}$, can also be prepared by gentle rehydration, where the lipid film is placed in a low ionic strength aqueous environment and liposomes form spontaneously over an extended period of time (days). The resulting LUVs vary in size and "pregnant" liposomes, where smaller vesicles are entrapped inside larger ones, are common. GUVs, which vary in size between $1 \mu \mathrm{m}$ and $100 \mu \mathrm{m}$, can be prepared by LUV fusion, however electroformation is used more frequently. ${ }^{43}$ Electroformation is a method where an electric field is externally applied to hydrating lipid films deposited on conductive surface such as indium tin oxide (ITO) coated glass or platinum wires. ${ }^{41}$ Liposomes have been successfully used as model 
systems for the investigation of mechanical and dynamic properties of membranes ${ }^{34,45-47}$ as well as for studies of peptide-bilayer interactions. ${ }^{48-52}$ Furthermore, liposome interactions have been studied to better understand native vesicle fusion ${ }^{53-55}$ and their internal cavities have been used as microreactors. ${ }^{56-58}$

\subsubsection{POLYMERSOMES}

Polymersomes are vesicles prepared by the self-assembly of amphiphilic polymeric building blocks. Polymer is a large molecule (Figure 1.10a) composed of repeating structural units defined as monomers (Figure 1.10b). Homopolymer is a polymer composed uniquely out of one type of monomer, whereas a polymer composed from more than one type of monomer is a copolymer (Figure 1.10c) and depending on the arrangement of the various monomers in the copolymer, specific copolymers are obtained (Figure 1.10d): alternating copolymer (1), statistical copolymer (2), block copolymer (3) and graft copolymer (4).

a)

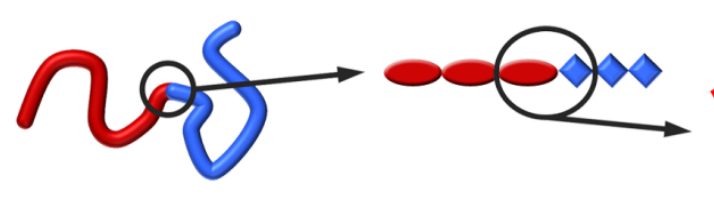

c)

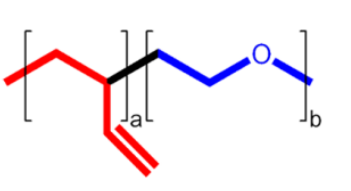

d) - A-B-A-B-A-B-A-B-A- (1)

$-\mathrm{A}-\mathrm{A}-\mathrm{A}-\mathrm{B}-\mathrm{B}-\mathrm{A}-\mathrm{B}-\mathrm{A}-\mathrm{A}-(2)$

$-A-A-A-A-A-B-B-B-B-(3)$

$-\mathrm{A}-\mathrm{A}-\mathrm{A}-\mathrm{A}-\mathrm{A}-\mathrm{A}-\mathrm{A}-\mathrm{A}-\mathrm{A}-(4)$

B-B-B-B B-B-B-B

Figure 1.10 Schematic representation of (a) block copolymer chain, (b) two types of repeating monomer units composing the polymer, (c) chemical structure of poly(butadiene)-block-poly(ethylene oxide) block copolymer and (d) various types of copolymers composed of two different monomers A and B: (1) alternating copolymer, (2) statistical copolymer, (3) block copolymer and (4) graft copolymer.

Similarly to liposomes, building blocks composing polymersomes have amphiphilic character where one block of the copolymer is hydrophilic and the other hydrophobic. When no solvent is present, block copolymers have been observed to adopt various ordered morphologies. Block copolymers self-assemble to form core-corona aggregates, where the corona-forming block is soluble while the core forming block is insoluble as it was first observed by Zhang and Eisenberg when investigating poly(styrene)- $b$ poly(acrylic acid) block copolymers. ${ }^{59}$ The self-assembly of block copolymers into defined aggregates, such as tubules, vesicles and deformed vesicles, was also observed with poly(styrene)-poly(ethylene oxide $)^{60}$ and poly(ethylene oxide)-poly(ethylethylene) ${ }^{61}$ copolymers. A scheme predicting the morphology of block copolymer aggregates, similar 
to the packing parameter defined by Israelachvili, ${ }^{5}$ was proposed by Discher et al. ${ }^{62}$ As with phospholipids where the head size and chain length determine the packing parameter, it is the relative mass (or volume fraction) of each block (hydrophilic and hydrophobic) of the copolymer that influences the morphology of the aggregate. It is the hydrophilic volume fraction $f$ of the block copolymer that is used to predict the aggregate's morphology (Figure 1.11). Micelles are formed from molecules with $f>45 \%$, copolymers with $f<25 \%$ result in inverted microstructures and when $f \approx 35 \% \pm 10 \%$ polymersome formation is expected. ${ }^{62}$

a) $f=25-45 \%$

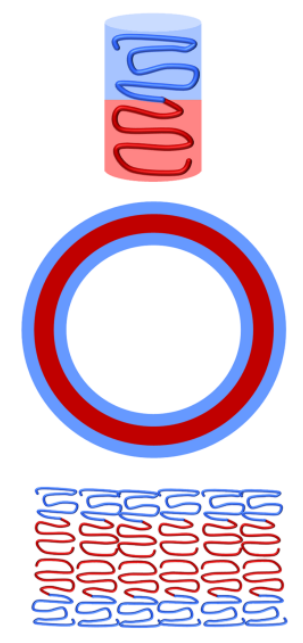

b)
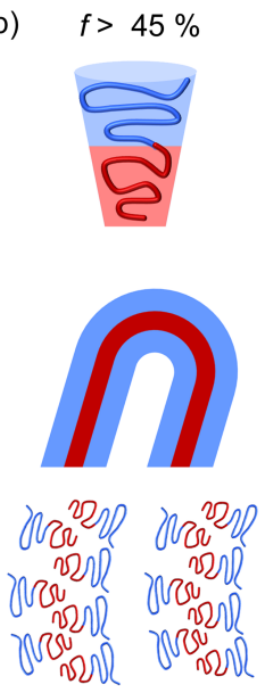

c)
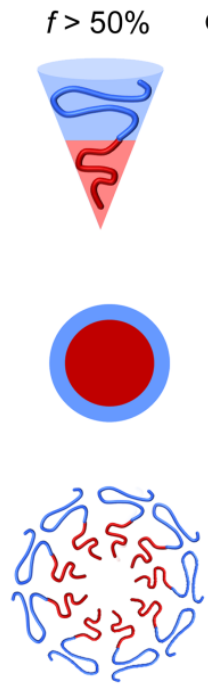

d)

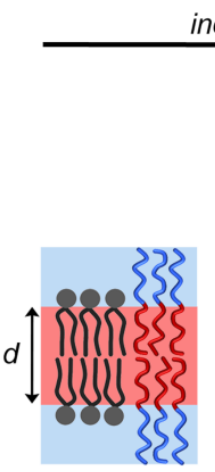

increasing $M_{W}$
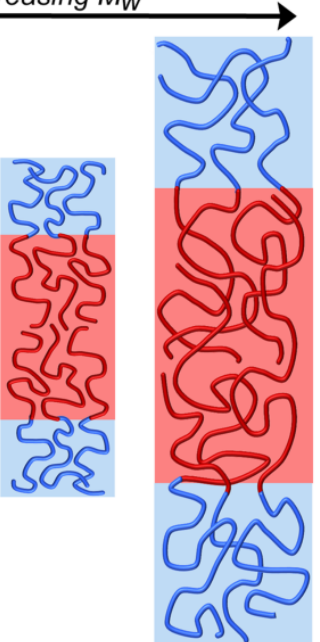

Figure 1.11 Relationship between the hydrophilic fraction $f$ of block copolymers and the structures formed from the aggregation of given block copolymers. (a) when $f=25-45 \%$ block copolymer has a cylindrical shape and vesicles are formed, (b) when $f>45 \%$ the block copolymer has a truncated cone shape and cylindrical vesicles are the preferred geometry and (c) when $f>50 \%$ the block copolymer assembles into micelles. In (d), the relationship between the increasing molecular weight of the polymer and the membrane thickness of polymersome is shown. (Figure adapted from Discher et al. ${ }^{62}$ )

In contrast to phospholipids, copolymer blocks composing the core of the polymersome membrane can interdigitate to form a dense core. ${ }^{63,64}$ Polymersome membrane thickness $d$ follows the copolymer molecular weight $M_{w}$ following the equation 1.9 determined experimentally and confirmed with coarse-grained molecular dynamic simulations. ${ }^{64,65}$

$$
d \sim M w^{b}(b \cong 0.55)
$$

with $b$ representing a scaling factor (for fully stretched polymer chains $b=1$ and for ideal random coils $b=1 / 2) .{ }^{64}$ Polymersomes are usually formed form diblock copolymers, but triblock copolymers have also been successfully used. Recently, it has been demonstrated 
that block copolymers which strongly hydrophobic block (ex. PB-PEO) form rigid impermeable membranes whereas triblock copolymers with mildly hydrophobic central blocks (ex. PEO-PPO-PEO) form more flexible and permeable membranes. ${ }^{60,66}$ The selfassembly into vesicles takes place when the block copolymer has the right volume fraction $(f=25-45 \%) .{ }^{44,67}$ Similarly to liposomes, polymersomes can be prepared by a wide range of techniques including polymer film rehydration, sonication and extrusion. ${ }^{68}$ Polymersomes are structurally similar to liposomes but exhibit increased mechanical stability and reduced permeability. ${ }^{44,69-71}$ It is the higher molecular weight of copolymers in comparison to phospholipids that confers liposomes their increased robustness, inertness, and lower permeability to water. As model membrane systems, polymersomes allow for a different approach to study membrane biophysics since the range of investigated properties can be considerably broadened as far as thermal, chemical, and mechanical properties are concerned. In terms of applications, the properties of polymersomes can be tailored by chemically adjusting the type, length and functionalization of the blocks of the copolymer. Biocompatibility is also an important issue and had been addressed when polymersomes were used as long-lived drug delivery vehicles. $^{72-75}$ In relation to drug delivery, controlled release of drugs has been accomplished with hydrolysable, oxidizable and switchable copolymers. ${ }^{71,76}$ PB- $b$-PEO block copolymer is a particularly interesting building block of polymersomes for potential drug delivery applications due to its biocompatibility and low toxicity. ${ }^{77-80}$ The hydrophilic interior of the vesicle can encapsulate hydrophilic molecules and, as it was recently shown by Mueller et al., hydrophobic ones can be incorporated into the hydrophobic part of the PB- $b$-PEO membrane. ${ }^{81}$ Generally, the suitability of polymersomes for drug delivery is strongly connected to both their mechanical stability and deformability. In polymer membranes, area dilatation of more than $40 \%$ can be easily reached before rupture occurs. ${ }^{64}$ However, a low bending modulus that allows the polymersome to be easily deformed once entered into the blood stream is as important as a high resistance against lateral dilatation.

The main interest of polymersome use is the potential use of such vesicles as carriers in programmable drug delivery systems. ${ }^{82}$ It was also shown that polymersomes can be used to study key biological membrane processes, for instance vesicle fusion and protein insertion. $^{63,83,84}$ 
Vesicles, liposomes and polymersomes, are good model membranes in terms of general membrane properties studies. However, the limitation of vesicles as model membranes is related to the whole vesicle experiments where the membrane mechanics measured are related to the total area of the vesicle surface instead of a chosen part of it.

\subsubsection{BLMs}

Black lipid membranes are the earliest model bilayer system, the term black referring to the disappearance of colorful interference bands after single bilayer membrane formation, when observed by optical microscopy. ${ }^{85,86}$ A black lipid membrane is prepared by spreading phospholipids dissolved in an organic solvent across an aperture, 50-100 $\mu \mathrm{m}$ in diameter, milled in a Teflon substrate which separates two chambers with aqueous solutions (Figure 1.12).

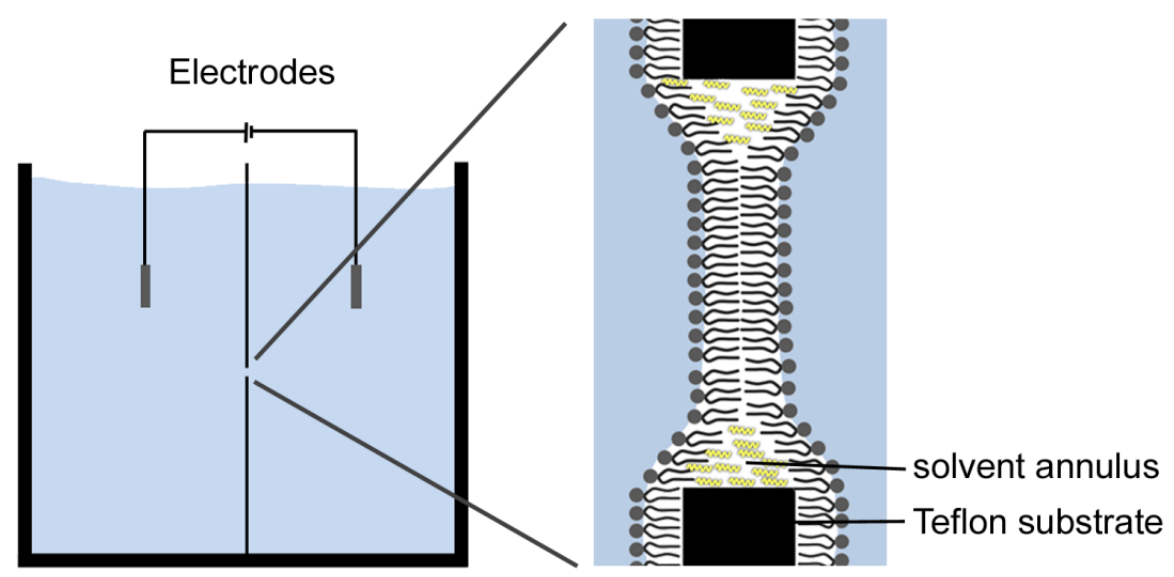

Figure 1.12 Schematic representation of a black lipid membrane (BLM) setup. A phospholipid bilayer is formed over a small aperture in a Teflon wall separating two chambers filled with aqueous solution.

During the thinning process, where the organic solvent is evaporating and the phospholipids are rearranging, a freestanding bilayer membrane, surrounded by aqueous environment is formed. Electrodes can be placed in the chambers separated by the BLM and allow for electrical characterization of the bilayer. When intact, a BLM has a G $\Omega$ resistance and $\mu \mathrm{F} / \mathrm{cm}^{2}$ capacitance. Ion channels can be formed in the BLMs by incorporation of peptides ${ }^{36,87}$, proteins $^{88,89}$ as well as antibiotics ${ }^{90}$ and the single channel events can be monitored. Recently, advances have been made in the preparation and study of BLMs, ${ }^{39,} 91$ however, the main drawbacks of BLMs include their limited long term 
stability, the presence of residual solvent which could affect protein function and the limited number of methods that can be used to detect their presence. ${ }^{92}$

\subsubsection{SOLID SUPPORTED MEMBRANES}

Solid supported membranes are prepared by spreading of liposomes on hydrophilic surfaces to produce bilayers, or on hydrophobic surfaces to produce mononolayers. ${ }^{93}, 94$ Both types of preparations have been extensively studied and resulted in better understanding of lipid membrane properties.

Flat surfaces used in the preparation of lipid monolayers and bilayers include mica, glass, oxidized silicon and other metals. ${ }^{92}$ The choice of solid substrate depends on the characterization technique that is used to study the model membrane. In general flatter surfaces are preferred such that nanometer height differences can be detected. In the case of lipid monolayers, Langmuir-Blodgett transfer is the technique which used most often. In a Langmuir-Blodgett trough, phospholipids are spread on water-air interface between two movable barriers which can be adjusted to create different surface pressures which are measured by a Wilhelmy plate. The monolayers created at the air-water interface can be transferred onto solid flat surfaces (i.e. mica or silicon) and further studied by ellipsometry, Brewster angle microscopy and AFM. Studies conducted on lipid monolayers have given insight into lipid arrangement depending on the type of lipids used, the ratios of different lipids and depending on surface pressures at which the monolayers were prepared. ${ }^{95}$

Vesicle spreading is another approach for preparation of solid supported membranes. A hydrophilic surface is exposed to a liposome solution and, by vesicle rupture and spreading, a bilayer membrane is formed on the surface. ${ }^{96}$ Surface characterization techniques mentioned above as well as others such as $\mathrm{NMR}^{97}, \mathrm{SPR}^{98}, \mathrm{FTIR}^{99}, \mathrm{QCM}^{100}$ and others can be used to determine membrane properties as long as the bilayer stays immersed in liquid. 
a)

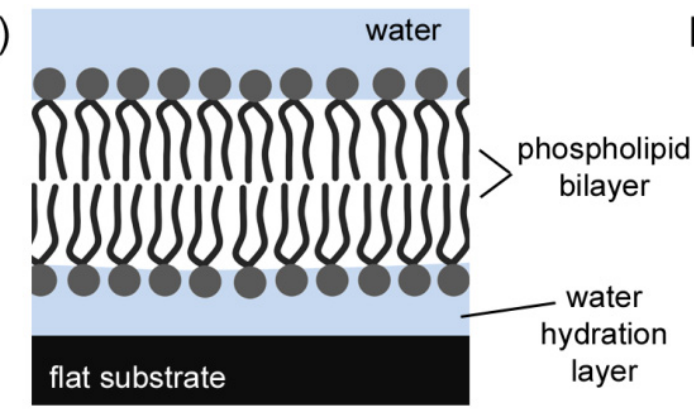

b)

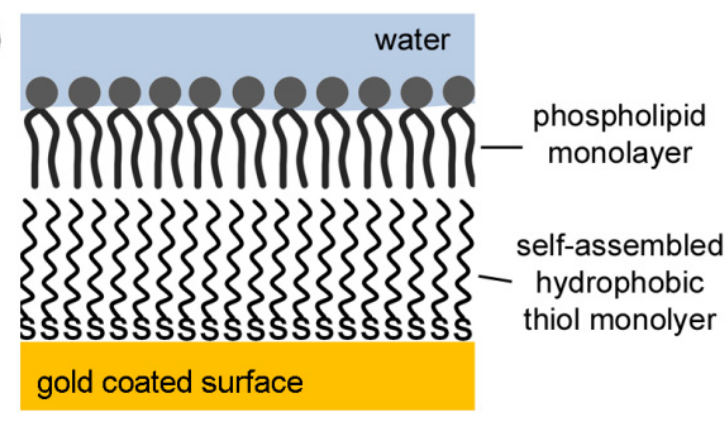

Figure 1.13 Schematic representation of (a) solid supported membrane where the phospholipid bilayer rests on a hydration layer of water molecules ${ }^{93,101}$ on the hydrophilic substrate and in (b) a solid supported hybrid membrane where a self-assembled monolayer of hydrophobic thiol molecules replaces the lower leaflet of the phospholipid bilayer.

Solid supported membranes have the advantage of being stable over $\mu \mathrm{m}-\mathrm{cm}$ distances, being fluid, and being suitable for characterization with various surface characterization techniques. Furthermore, their composition can be easily controlled. For more than 20 years, solid supported membranes have been the most commonly used membrane model for various membrane studdies. ${ }^{55}$ However, the proximity of the membrane to the solid substrate can be problematic with respect to transmembrane protein insertion and its proper functioning once it is spanning the membrane. The drawback associated with the membrane-substrate distance can be remedied by including a soft polymer layer between the substrate and the membrane. ${ }^{102}$ Hydrated polymer cushions which mimic extracellular matrix, can be used as space creating, lubricating layers between the membrane and the surface. Another strategy to control the distance between the membrane and the surface is to use lipids with polymer-modified head groups to create polymer-tethered membranes. By incorporating them into the lipid layer a larger distance between the membrane and the surface is formed. ${ }^{92,} 94$ Such tethers are based on poly(ethylene oxide) and oligopeptides with thiol groups. Since the length of the tethers can be adjusted synthetically and the lateral spacing of the tethers can be controlled, the membranesubstrate distance as well as the viscosity of the tethered layer can be controlled which in turn affects the lateral diffusion of and function of transmembrane proteins. ${ }^{103,104}$ 
a)

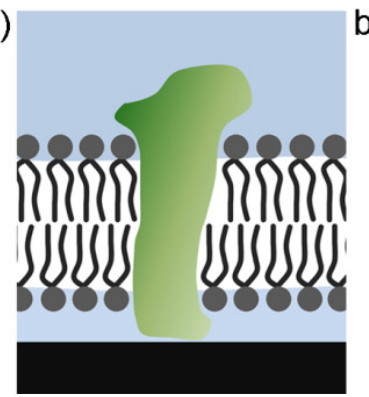

b)

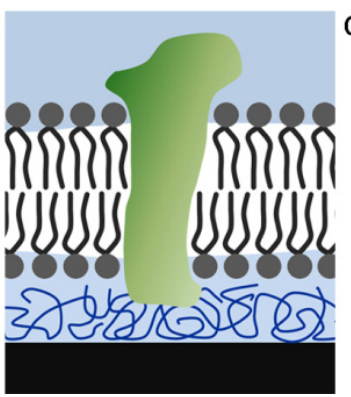

c)

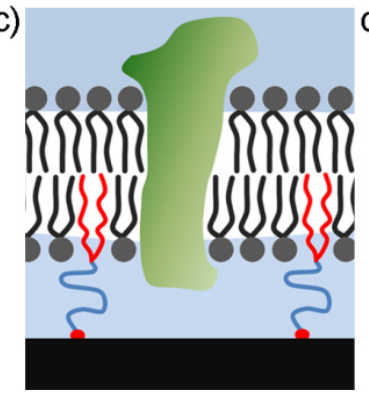

d)

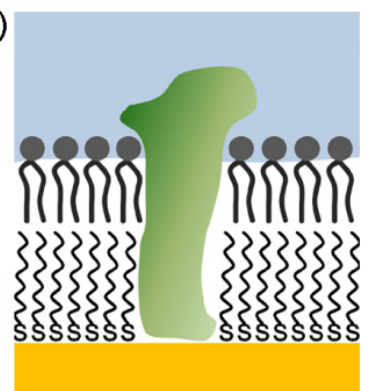

Figure 1.14 Schematic representation showing a transmembrane protein within: (a) solid supported membrane, (b) polymer-cushioned membrane, (c) polymer-tethered membrane (d) hybrid membrane prepared on hydrophobic self-assembled monolayer.

\subsubsection{PORE-SPANNING MEMBRANES}

To combine the free-standing nature of BLMs together with the mechanical stability of solid supported membranes, the use of porous substrates in the preparation of model membrane system became the focus of many research groups. ${ }^{105-108}$ Similarly to solid supported membranes, the membrane is supported by the substrate however, the substrate is porous. The combination of partial support from the interpore surface together with the horizontal free-standing character of the membrane results in a model membrane which keeps the qualities of the models currently available and eliminates some of their flaws. Micro and nanosized pores such as anodized porous alumina or micromachined silicon nitride substrates have been used as potential porous substrates. ${ }^{39,} 109$ The major difficulty with preparation of pore-spanning membranes lies in the actual spanning of the pores with the membrane. Naturally, a membrane prefers to form on a substrate instead of a void. Therefore, during the spreading, the membrane tends to coat the interpore surface and the interior of the pores. To overcome the tendency of the membrane to avoid spanning the pores, chemical functionalization strategies of the porous surface such as self-assembled monolayers $(\mathrm{SAM})^{35,} 109$ or silanization ${ }^{110}$ procedures have been employed. Self-assembly of functional thiolates on gold surfaces is a practical and commonly used approach in surface functionalization. ${ }^{11-113}$ In summary, when a clean gold surface is exposed to an ethanolic thiol solution, a SAM is formed on the gold surface through formation of thiolates according to equations $1.10 \mathrm{a}$ or $1.10 \mathrm{~b} .^{112,114}$ 
The chemical nature of the SAM is the same as the chemical nature of the molecules used to create the SAM, allowing for a broad range of possible surface functionalities. Besides SAMs, other membrane preparation techniques have been developed, all resulting in pore-spanning membranes with defined properties, advantages and drawbacks. ${ }^{35,108,109,115}$ One of the main advantages of pore-spanning membranes is the lack of solid support which is beneficial for transmembrane proteins and allows for the study of membrane mechanics by indentation.

\subsubsection{HYBRID NANO-BLMS}

One type of pore-spanning membranes developed with great success is the hybrid micro or nano-BLMs. These pore-spanning membranes are prepared by the same painting technique used for the preparation of original BLMs. ${ }^{116}$ Instead of one aperture separating two chambers, substrate with a pore array is used. To prepare pore-spanning membranes, the porous substrate is first gold-coated and then chemically functionalized with a hydrophobic self-assembled monolayer (SAM) of a hydrophobic thiol. The SAM creates a hydrophobic monolayer on the inter-pore surface (pore-rim) and the pore remains empty. The following step is to "paint" lipids dissolved in organic solvent over the porous surface and wait for the lipids to thin out and form lipid bilayers over the pore area. The resulting pore-spanning membrane is a phospholipid bilayer spanning the pores and a hybrid membrane consisting of a SAM leaflet and phospholipid leaflet covering the interpore surface, hence the hybrid designation. ${ }^{35,109,115}$ 


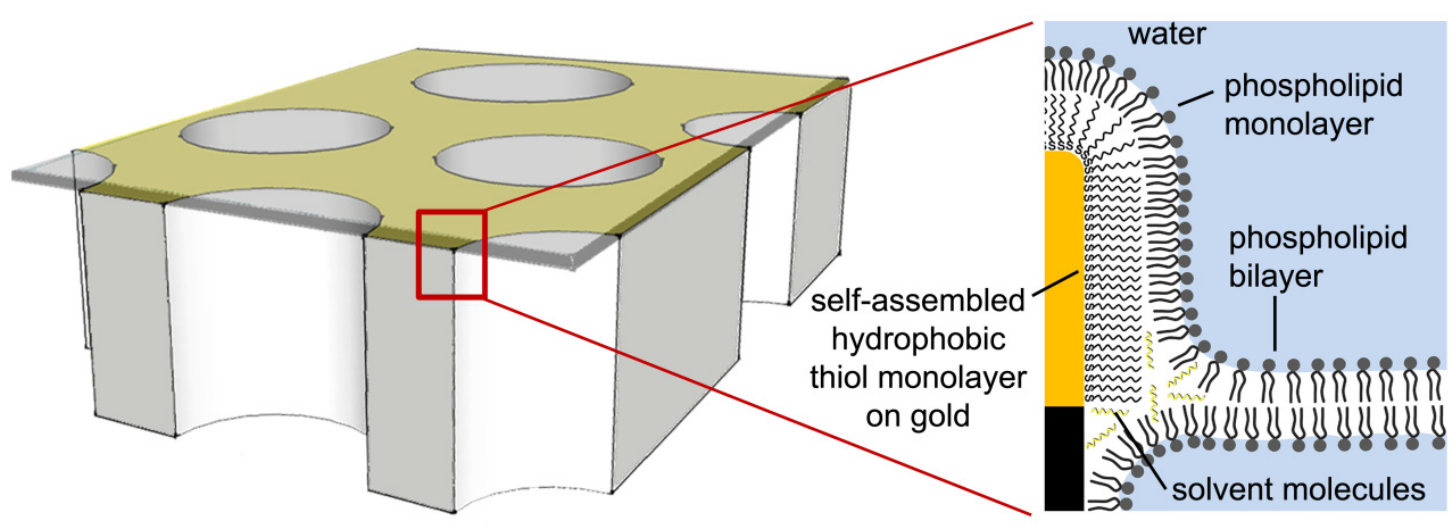

Figure 1.15 Schematic representation of a hybrid pore-spanning membrane. Octanethiol SAM forms on the interpore surface and on the edge of the pore. Phospholipids painted over the porous surface arrange during the thinning process to create a bilayer membrane over the pore and a hybrid membrane on the pore-rim.

Hybrid micro/nano-BLMs prepared by the painting technique have the disadvantage of containing trace amounts of organic solvent. To circumvent this problem, hybrid micro/nano-BLMs can be prepared by vesicle spreading. The surface is functionalized with a SAM as in the "painting" approach but, instead of painting the lipids over the pores, hybrid membranes are created by rupture of liposomes. When prepared by vesicle rupture, fewer pore-spanning membranes are obtained due to the stresses created during vesicle delamination, a step necessary to accommodate the hydrophobic pore-rims.

The advantages of hybrid micro/nano-BLMs lie in the planar nature of the membranes prepared as well as in increased mechanical stability of the pore-spanning membranes in contrast to original BLMs. The hybrid character of the membrane on the pore rims is an important disadvantage of these membranes. The attachment of the membrane to the pore rims through the interaction of the SAM with top phospholipid leaflet is responsible for the inherent tension of the pore-spanning membranes. ${ }^{35}$ Furthermore, the diffusion of lipid molecules is restricted to the top leaflet of the bilayer over the pore-rims, which reduces the overall diffusion of the lipids over the entire porous surface. Amid some flaws, hybrid nano-BLMs on nanoporous alumina have been successfully studied by electrical impedance spectroscopy ${ }^{117}$ and single channel recordings of gramacidin ${ }^{118}$ as well as of $\mathrm{OmpF}^{39}$ have been recorded. Furthermore, DPhPC hybrid micro-BLMs on silicon substrates have been proven suitable for study by impedance spectroscopy and to perform fluorescence recovery after photobleaching experiments. ${ }^{109}$ Experiments were also performed on hybrid mirco/nano-BLMs prepared by vesicle spreading. In one study, DPhPC:DOPC (60:40) vesicles were spread on alumina. Membrane spreading and pore 
sealing were confirmed by the inserting bacteriodophsin proton pump into the membrane and by testing its functionality. ${ }^{115}$ Finally, AFM experiments have been used to determine the highly tensed nature of the hybrid pore-spanning membranes. ${ }^{35}$

\subsubsection{FREE-FLOWING PORE SPANNING MEMBRANES}

The potential applicability of pore-spanning membranes in biophysical research has sparked interest in the elimination of flaws still existing with the preparation of these membranes. The two main concerns are: the presence of trace amounts of organic solvent arising from the painting preparation method and the inherent tension of the membranes stemming from their hybrid character. To solve these problems, preparation of porespanning membranes by vesicle rupture has been favored and the hydrophobic pretreatment of the pore-rims was replaced by different surface functionalities or pretreatment. Several research groups have focused on improving the pore-spanning membrane preparation methods and made substantial advances.

In 2009, the Naumann research group prepared silicon nitride porous substrates with 50-600 nm diameter pores by focused ion beam. DPhPC GUVs were then added and maneuvered with a tip of a micromanipulator in order to spread the liposome over the pores and create pore-spanning membranes. Measurement of ionic currents through gramicidin channels inserted into this membrane was accomplished showing the potential application of these model membranes as a platform for membrane protein research. ${ }^{38}$

Further advances in the preparation of free-standing membranes came from the Höök research group in 2010. Pore-spanning membranes were created in a microfluidic setup by shear forces arising from a bulk flow over solid supported lipid bilayers. The porous substrate used for pore-spanning membrane preparation was $\mathrm{SiO}_{2}$ and the pore diameter was of $80 \mathrm{~nm}$. Encapsulation of carboxyfluorescein in the pores was performed to confirm the pore-spanning nature of the membranes. ${ }^{119}$ One of the latest reports of freestanding pore-spanning membrane preparation was reported by the research group of Sang-Hyun Oh. ${ }^{108}$ In their work, eggPC vesicles were spread on nanopores formed in gold/silicon nitride films. To prepare the porous substrates with $200 \mathrm{~nm}$ diameter pores, $\mathrm{Si}_{3} \mathrm{~N}_{4}$ layer covered with $200 \mathrm{~nm}$ thick gold layer, deposited by electron beam evaporator, was milled by focused ion beam. Besides fluorescence microscopy, pore-spanning 
membrane presence was confirmed by alpha hemolisin addition and SPR monitoring of the changes caused to the membrane.

In summary, model membranes have been developed to mimic the native cell membranes in order to study membrane properties without the inconvenience of the native cell membrane complexity. All of the model membranes presented have advantages and disadvantages depending on the type of study that is carried out. Until now, no preparation method can be defined as perfect thus the continued research in that theme. 


\subsection{STUdy OF MODEL MEMBRANES}

The study of surface interactions is challenging because of the small distances at which they are taking effect as well as their small magnitude. Characterization techniques such as fluorescence microscopy, impedance spectroscopy, FTIR, x-ray and neutron scattering, SPR and others can be adapted to study the physical properties of model membranes introduced in the previous section. In terms of mechanical properties however, investigation techniques are limited and include surface force apparatus, micropipette aspiration and atomic force microscopy.

Surface force apparatus (SFA) (Figure 1.16a) is a technique developed by Tabor, Winterton and Israelachvili and is based on the interaction of two crossed silica cylinders whose separation distance is measured by multiple beam interferometry. ${ }^{120-122}$ Interaction forces are measured between two crossed, atomically smooth mica cylinders of roughly 1 $\mathrm{cm}$ radius. The separation between the two cylinders is measured by an optical technique using multiple beam interference fringes. Studies with SFA have elucidated the fundamental interactions occurring between surfaces including van der Waals forces, hydrophobic/hydrophilic forces and others. ${ }^{123}$ The limitation of this technique is based on the experimental setup where only solid (mica) supported membranes can be studied.

a)

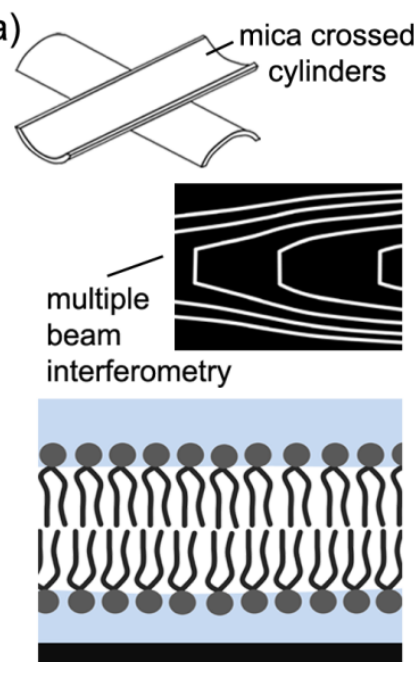

b)

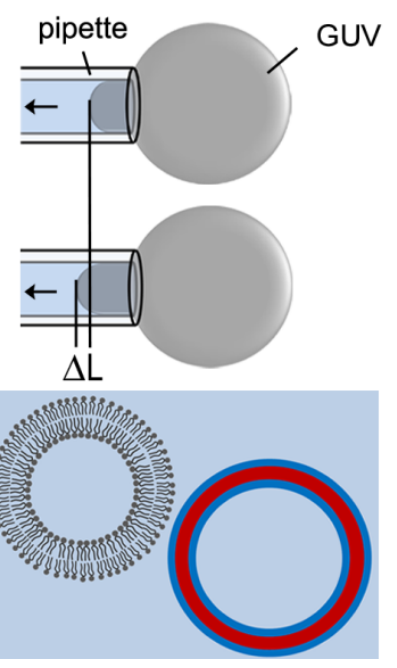

c) photodetector
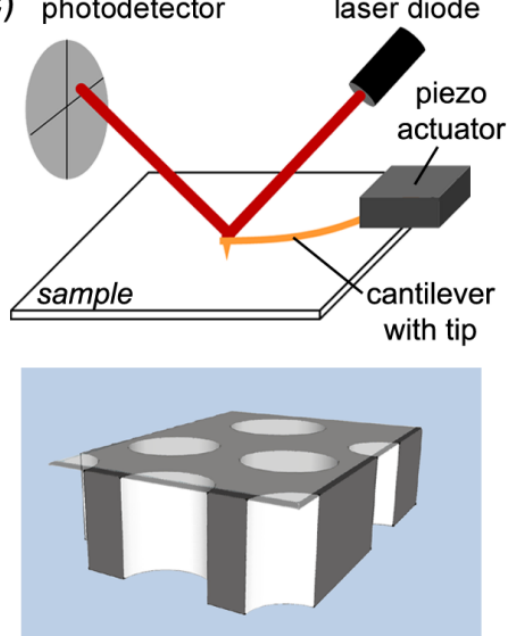

Figure 1.16 Experimental techniques used for investigation of mechanical properties of surfaces. (a) surface force apparatus technique suitable for solid-supported membranes (b) micropipette aspiration techniques used to investigate vesicles and (c) atomic force microscopy which can be used to investigate pore-spanning membranes. 
Micropipette aspiration technique is a relatively simple method used to study the mechanical properties of membranes (Figure 1.16b). A glass micropipette with a know inner diameter is brought into close contact with a vesicle and because of negative pressure which is applied through the pipette, the vesicle is sucked into the pipette. Videomicroscopy is used to monitor the extension of the vesicle into the pipette and the change in area of the vesicle projected into the pipette is calculated. ${ }^{20}$

By knowing the micropipette's diameter, the applied suction and by calculating the length of the vesicle projected into the pipette, one can calculate membrane tension according to formula:

$$
\tau=\frac{\Delta P R_{\mathrm{p}}}{2\left(1-\frac{R_{\mathrm{p}}}{R_{\mathrm{v}}}\right)}
$$

where $\tau$ represents tension of the membrane, $\Delta \mathrm{P}$ is the change in the pressure exerted on the vesicle and $R_{p}$ and $R_{v}$ are the radius of the pipette and radius of the vesicle, respectively. ${ }^{20}$ Furthermore, the change in the projection length $(\Delta \mathrm{L})$ is related to the apparent area strain $(\alpha)$ through the following relationship:

$$
\alpha=\frac{\Delta A}{A_{0}} \approx \frac{1}{2}\left\{\left(\frac{R_{\mathrm{p}}}{R_{\mathrm{v}}}\right)^{2}-\left(\frac{R_{\mathrm{p}}}{R_{\mathrm{v}}}\right)^{3}\right\} \frac{\Delta L}{R_{\mathrm{p}}}
$$

where the apparent surface area change $(\Delta \mathrm{A})$ is obtained from the surface area at initial membrane tension $\left(\mathrm{A}_{0}\right)$. When the experiments are performed in the low tension range, the bending modulus ( $\kappa)$ of the membrane is extracted from the slope of a line obtained by plotting the natural log of tension versus the apparent area strain and multiplying this slope by $\mathrm{k}_{\mathrm{b}} \mathrm{T} / 8 \pi$. Micropipette aspiration technique is used to determine the mechanical properties of membranes however, its applicability is limited to vesicles.

Optical microscopy and scanning probe microscopy are two characterization techniques which are well adapted to study pore-spanning membranes. Besides detecting fluorescently-labeled membranes on porous substrates, fluorescence microscopy is used to obtain diffusion constants of lipids by performing fluorescence recovery after photobleaching experiments. 
Atomic force microscopy (AFM) can be used to image pore-spanning membranes in biological environments and to perform site-specific studies of membrane mechanics (Figure 1.16c). Invented in 1986 by Binnig, Quate and Gerber, AFM is a characterization technique where a sharp tip (probe) is scanned over the surface and its interactions with the surface are recorded in the form of a topographic image. ${ }^{124}$

Force versus distance experiments can be performed to investigate the forces acting between the tip and the surface. The cantilever is moved in the z-axis and the cantilever deflection versus piezo displacement is registered. The deflection is converted into a force by multiplying it with the spring constant of the cantilever. AFM force versus distance curves allow for local mechanical investigation of surfaces because the interaction is limited to the area in contact with the AFM tip. 


\subsection{SCOPE OF THESIS}

The main objectives of this work were 1) to develop procedures for preparation of model pore-spanning membranes which would mimic native membranes and 2) to study the mechanical properties of these model pore-spanning membranes with the goal of establishing reference values for further experiments which will involve more complex models and native systems.

The first part of the experimental work was focused on the preparation and characterization of model pore-spanning polymer membranes prepared from block copolymer poly(butadiene)- $b$-poly(ethylene oxide) (PB- $b$-PEO) vesicles. Their porespanning character was investigated by fluorescence microscopy as well as atomic force microscopy imaging. After successful preparation of pore-spanning membranes on porous silicon nitride substrates, their mechanical properties were investigated by force indentation experiments. Furthermore, cross-linking of the hydrophobic core of the polymer membranes was performed to verify if the entanglement of the polymer chains has an effect on the mechanical response.

The second part of the experimental work was aimed at the preparation of model phospholipid pore-spanning membranes from giant unilamellar (GUVs) vesicles. Surface functionalization of porous silicon nitride substrates was performed to induce vesicles rupture and pore-spanning membrane formation. Fluorescence microscopy, confocal laser scanning microscopy (CLSM) and fluorescence recovery after photobleaching (FRAP) were used to confirm pore-spanning membrane formation, single bilayer structure of the membrane and the fluidity of the membranes on the porous substrates, respectively. Force indentation experiments were performed to determine the mechanical response of the lipid membranes to applied load. Furthermore, isopropanol was added to the environment of the membranes and subsequent change in membrane tension was observed. 


\subsection{REFERENCES}

1. Karp, G., Cell and molecular biology. $3^{\text {rd }}$ ed.; John Wiley \& Sons Inc.: New York, 2002.

2. Alberts, B.; Johnson, A.; Lewis, J.; Raff, m.; Roberts, K.; Walter, P., Molecular biology of the cell. 4th ed.; Garland Science: New York, 2002.

3. Stryer, L., Biochemistry. $4^{\text {th }}$ ed.; W. H. Freeman and Company: New York, 1995.

4. White, S. H.; Wimley, W. C., Membrane protein folding and stability: Physical principles. Annual Review of Biophysics and Biomolecular Structure 1999, 28, 319-365.

5. Israelachvili, J., Intermolecular and surface forces. Academic Press, Elsevier: Amsterdam, 1991.

6. Boal, D., Mechanics of the cell. Cambridge University Press: Cambridge, 2001.

7. Sprong, H.; van der Sluijs, P.; van Meer, G., How proteins move lipids and lipids move proteins. Nat Rev Mol Cell Biol 2001, 2, (7), 504-513.

8. Singer, S. J.; Nicolson, G. L., The fluid mosaic model of the structure of cell membranes. Science 1972, 175, (4023), 720-731.

9. Kusumi, A.; Sako, Y.; Yamamoto, M., Confined lateral diffusion of membrane receptors as studied by single particle tracking (Nanovid Microscopy). Effects of calcium-induced differentiation in cultured epithelial cells. Biophysical Journal 1993, 65, (5), 2021-2040.

10. Morone, N.; Fujiwara, T.; Murase, K.; Kasai, R. S.; Ike, H.; Yuasa, S.; Usukura, J.; Kusumi, A., Three-dimensional reconstruction of the membrane skeleton at the plasma membrane interface by electron tomography. Journal of Cell Biology 2006, 174, (6), 851-862.

11. Ritchie, K.; Iino, R.; Fujiwara, T.; Murase, K.; Kusumi, A., The fence and picket structure of the plasma membrane of live cells as revealed by single molecule techniques. Molecular Membrane Biology 2003, 20, (1), 13-18.

12. Fujiwara, T.; Ritchie, K.; Murakoshi, H.; Jacobson, K.; Kusumi, A., Phospholipids undergo hop diffusion in compartmentalized cell membrane. The Journal of Cell Biology 2002, 157, (6), 10711082.

13. Kusumi, A.; Nakada, C.; Ritchie, K.; Murase, K.; Suzuki, K.; Murakoshi, H.; Kasai, R. S.; Kondo, J.; Fujiwara, T., Paradigm shift of the plasma membrane concept from the two-dimensional continuum fluid to the partitioned fluid: High-speed single-molecule tracking of membrane molecules. Annual Review of Biophysics and Biomolecular Structure 2005, 34, 351-U54.

14. Simons, K.; Ikonen, E., Functional rafts in cell membranes. Nature 1997, 387, (6633), 569-572.

15. Simons, K.; Gerl, M. J., Revitalizing membrane rafts: New tools and insights. Nat Rev Mol Cell Biol 2010, 11, (10), 688-699.

16. Simons, K.; Toomre, D., Lipid rafts and signal transduction. Nature Reviews Molecular Cell Biology 2000, 1, (1), 31-39.

17. Dietrich, C.; Bagatolli, L. A.; Volovyk, Z. N.; Thompson, N. L.; Levi, M.; Jacobson, K.; Gratton, E., Lipid rafts reconstituted in model membranes. Biophysical Journal 2001, 80, (3), 1417-1428.

18. Shaw, A. S., Lipid rafts: Now you see them, now you don't. Nature Immunology 2006, 7, (11), 1139-1142.

19. Munro, S., Lipid rafts: Elusive or illusive? Cell 2003, 115, (4), 377-388. 
20. Rawicz, W.; Olbrich, K. C.; McIntosh, T.; Needham, D.; Evans, E., Effect of chain length and unsaturation on elasticity of lipid bilayers. Biophysical Journal 2000, 79, (1), 328-339.

21. Apodaca, G., Modulation of membrane traffic by mechanical stimuli. American Journal of Physiology-Renal Physiology 2002, 282, (2), F179-F190.

22. Dai, J. W.; TingBeall, H. P.; Sheetz, M. P., The secretion-coupled endocytosis correlates with membrane tension changes in rbl 2 h3 cells. Journal of General Physiology 1997, 110, (1), 1-10.

23. Mooseker, M. S., Organization, chemistry, and assembly of the cytoskeletal apparatus of the intestinal brush-border. Annual Review of Cell Biology 1985, 1, 209-241.

24. Nambiar, R.; McConnell, R. E.; Tyska, M. J., Control of cell membrane tension by myosin-i. Proceedings of the National Academy of Sciences of the United States of America 2009, 106, (29), 11972-11977.

25. Raucher, D.; Sheetz, M. P., Cell spreading and lamellipodial extension rate is regulated by membrane tension. Journal of Cell Biology 2000, 148, (1), 127-136.

26. Sheetz, M. P., Cell control by membrane-cytoskeleton adhesion. Nature Reviews Molecular Cell Biology 2001, 2, (5), 392-396.

27. Sheetz, M. P.; Dai, J. W., Modulation of membrane dynamics and cell motility by membrane tension. Trends in Cell Biology 1996, 6, (3), 85-89.

28. Togo, T.; Krasieva, T. B.; Steinhardt, R. A., A decrease in membrane tension precedes successful cell-membrane repair. Molecular Biology of the Cell 2000, 11, (12), 4339-4346.

29. Dai, J. W.; Sheetz, M. P., Membrane tether formation from blebbing cells. Biophysical Journal 1999, 77, (6), 3363-3370.

30. Dimova, R.; Aranda, S.; Bezlyepkina, N.; Nikolov, V.; Riske, K. A.; Lipowsky, R., A practical guide to giant vesicles. Probing the membrane nanoregime via optical microscopy. Journal of Physics-Condensed Matter 2006, 18, (28), S1151-S1176.

31. Evans, E.; Rawicz, W., Entropy-driven tension and bending elasticity in condensed-fluid membranes. Physical Review Letters 1990, 64, (17), 2094.

32. Evans, E.; Yeung, A., Hidden dynamics in rapid changes of bilayer shape. Chemistry and Physics of Lipids 1994, 73, (1-2), 39-56.

33. Evans, E. A.; Hochmuth, R. M., Membrane viscoelasticity. Biophysical Journal 1976, 16, (1), 111.

34. Garcia-Manyes, S.; Redondo-Morata, L.; Oncins, G.; Sanz, F., Nanomechanics of lipid bilayers: Heads or tails? Journal of the American Chemical Society 2010, 132, (37), 12874-12886.

35. Mey, I.; Stephan, M.; Schmitt, E. K.; Muller, M. M.; Ben Amar, M.; Steinem, C.; Janshoff, A., Local membrane mechanics of pore-spanning bilayers. Journal of the American Chemical Society 2009, 131, (20), 7031-7039.

36. Bamberg, E.; Alpes, H.; Apell, H. J.; Bradley, R.; Härter, B.; Quelle, M. J.; Urry, D. W., Formation of ionic channels in black lipid membranes by succinic derivatives of gramicidin a. Journal of Membrane Biology 1979, 50, (3), 257-270. 
37. Gonzalez-Perez, A.; Stibius, K. B.; Vissing, T.; Nielsen, C. H.; Mouritsen, O. G., Biomimetic triblock copolymer membrane arrays: A stable template for functional membrane proteins. Langmuir 2009, 25, (18), 10447-10450.

38. Kresak, S.; Hianik, T.; Naumann, R. L. C., Giga-seal solvent-free bilayer lipid membranes: From single nanopores to nanopore arrays. Soft Matter 2009, 5, (20), 4021-4032.

39. Schmitt, E. K.; Vrouenraets, M.; Steinem, C., Channel activity of OmpF monitored in nano-BLMs. Biophysical Journal 2006, 91, (6), 2163-2171.

40. Lasic, D. D., The mechanism of vesicle formation. Biochemical Journal 1988, 256, (1), 1-11.

41. Angelova, M. I.; Dimitrov, D. S., Liposome electroformation. Faraday Discussions 1986, 81, 303311.

42. Bagatolli, L. A.; Parasassi, T.; Gratton, E., Giant phospholipid vesicles: Comparison among the whole lipid sample characteristics using different preparation methods: A two photon fluorescence microscopy study. Chemistry and Physics of Lipids 2000, 105, (2), 135-147.

43. Walde, P.; Cosentino, K.; Engel, H.; Stano, P., Giant vesicles: Preparations and applications. ChemBioChem 2010, 11, (7), 848-865.

44. Antonietti, M.; Förster, S., Vesicles and liposomes: A self-assembly principle beyond lipids. Advanced Materials 2003, 15, (16), 1323-1333.

45. Ariola, F. S.; Li, Z.; Cornejo, C.; Bittman, R.; Heikal, A. A., Membrane fluidity and lipid order in ternary giant unilamellar vesicles using a new bodipy-cholesterol derivative. Biophysical Journal 2009, 96, (7), 2696-2708.

46. Demchenko, A. P.; Mély, Y.; Duportail, G.; Klymchenko, A. S., Monitoring biophysical properties of lipid membranes by environment-sensitive fluorescent probes. Biophysical Journal 2009, 96, (9), 3461-3470.

47. Vitkova, V.; et al., Deformation of vesicles flowing through capillaries. EPL (Europhysics Letters) 2004, 68, (3), 398.

48. Kriegsmann, J.; Gregor, I.; von der Hocht, I.; Klare, J.; Engelhard, M.; Enderlein, J.; Fitter, J., Translational diffusion and interaction of a photoreceptor and its cognate transducer observed in giant unilamellar vesicles by using dual-focus fcs. ChemBioChem 2009, 10, (11), 1823-1829.

49. Mally, M.; Majhenc, J.; Svetina, S.; Zeks, B., Mechanisms of equinatoxin ii-induced transport through the membrane of a giant phospholipid vesicle. Biophysical Journal 2002, 83, (2), 944-953.

50. Takeda, S.; Saitoh, A.; Furuta, M.; Satomi, N.; Ishino, A.; Nishida, G.; Sudo, H.; Hotani, H.; Takiguchi, K., Opening of holes in liposomal membranes is induced by proteins possessing the ferm domain. Journal of Molecular Biology 2006, 362, (3), 403-413.

51. Tamba, Y.; Yamazaki, M., Single giant unilamellar vesicle method reveals effect of antimicrobial peptide magainin 2 on membrane permeability. Biochemistry 2005, 44, (48), 15823-15833.

52. Yu, Y.; Vroman, J. A.; Bae, S. C.; Granick, S., Vesicle budding induced by a pore-forming peptide. Journal of the American Chemical Society 2010, 132, (1), 195-201.

53. Haluska, C. K.; Riske, K. A.; Marchi-Artzner, V. r.; Lehn, J.-M.; Lipowsky, R.; Dimova, R., Time scales of membrane fusion revealed by direct imaging of vesicle fusion with high temporal resolution. Proceedings of the National Academy of Sciences 2006, 103, (43), 15841-15846. 
54. Heuvingh, J.; Pincet, F.; Cribier, S., Hemifusion and fusion of giant vesicles induced by reduction of inter-membrane distance. The European Physical Journal E: Soft Matter and Biological Physics 2004, 14, (3), 269-276.

55. Tanaka, M.; Sackmann, E., Polymer-supported membranes as models of the cell surface. Nature 2005, 437, (7059), 656-663.

56. Chiu, D. T.; Wilson, C. F.; Ryttsen, F.; Stromberg, A.; Farre, C.; Karlsson, A.; Nordholm, S.; Gaggar, A.; Modi, B. P.; Moscho, A.; Garza-Lopez, R. A.; Orwar, O.; Zare, R. N., Chemical transformations in individual ultrasmall biomimetic containers. Science 1999, 283, (5409), 1892 1895.

57. Kulin, S.; Kishore, R.; Helmerson, K.; Locascio, L., Optical manipulation and fusion of liposomes as microreactors. Langmuir 2003, 19, (20), 8206-8210.

58. Yang, P.; Lipowsky, R.; Dimova, R., Nanoparticte formation in giant vesicles: Synthesis in biomimetic compartments. Small 2009, 5, (18), 2033-2037.

59. Zhang, L.; Eisenberg, A., Multiple morphologies of "Crew-cut" Aggregates of polystyrene-bpoly(acrylic acid) block copolymers. Science 1995, 268, (5218), 1728-1731.

60. Yu, K.; Eisenberg, A., Bilayer morphologies of self-assembled crew-cut aggregates of amphiphilic PS-b-PEO diblock copolymers in solution. Macromolecules 1998, 31, (11), 3509-3518.

61. Hajduk, D. A.; Kossuth, M. B.; Hillmyer, M. A.; Bates, F. S., Complex phase behavior in aqueous solutions of poly(ethylene oxide)-poly(ethylethylene) block copolymers. The Journal of Physical Chemistry B 1998, 102, (22), 4269-4276.

62. Discher, D. E.; Ahmed, F., Polymersomes. Annual Review of Biomedical Engineering 2006, 8, (1), 323-341.

63. Discher, B. M.; Won, Y.-Y.; Ege, D. S.; Lee, J. C. M.; Bates, F. S.; Discher, D. E.; Hammer, D. A., Polymersomes: Tough vesicles made from diblock copolymers. Science 1999, 284, (5417), 1143-1146.

64. Bermudez, H.; Brannan, A. K.; Hammer, D. A.; Bates, F. S.; Discher, D. E., Molecular weight dependence of polymersome membrane structure, elasticity, and stability. Macromolecules 2002, 35, (21), 8203-8208.

65. Srinivas, G.; Discher, D. E.; Klein, M. L., Self-assembly and properties of diblock copolymers by coarse-grain molecular dynamics. Nat Mater 2004, 3, (9), 638-644.

66. Rodriguez-Garcia, R.; Mell, M.; Lopez-Montero, I.; Netzel, J.; Hellweg, T.; Monroy, F., Polymersomes: Smart vesicles of tunable rigidity and permeability. Soft Matter 2011, 7, (4), 15321542.

67. Maskos, M., Influence of the solvent and the end groups on the morphology of cross-linked amphiphilic poly(1,2-butadiene)-b-poly(ethylene oxide) nanoparticles. Polymer 2006, 47, (4), 1172-1178.

68. Lee, J. C. M.; Bermudez, H.; Discher, B. M.; Sheehan, M. A.; Won, Y.-Y.; Bates, F. S.; Discher, D. E., Preparation, stability, and in vitro performance of vesicles made with diblock copolymers. Biotechnology and Bioengineering 2001, 73, (2), 135-145. 
69. Chécot, F.; Lecommandoux, S.; Klok, H. A.; Gnanou, Y., From supramolecular polymersomes to stimuli-responsive nano-capsules based on poly(diene-b-peptide) diblock copolymers. European Physical Journal E: Soft Matter 2003, 10, (1), 25-35.

70. Lecommandoux, S.; Sandre, O.; Chécot, F.; Rodriguez-Hernandez, J.; Perzynski, R., Magnetic nanocomposite micelles and vesicles. Advanced Materials 2005, 17, (6), 712-718.

71. Onaca, O.; Enea, R.; Hughes, D., W.; Meier, W., Stimuli-responsive polymersomes as nanocarriers for drug and gene delivery. Macromolecular Bioscience 2009, 9, (2), 129-139.

72. Choucair, A.; Soo, P. L.; Eisenberg, A., Active loading and tunable release of doxorubicin from block copolymer vesicles. Langmuir 2005, 21, (20), 9308-9313.

73. Discher, B. M.; Bermudez, H.; Hammer, D. A.; Discher, D. E.; Won, Y. Y.; Bates, F. S., Crosslinked polymersome membranes: Vesicles with broadly adjustable properties. Journal of Physical Chemistry B 2002, 106, (11), 2848-2854.

74. Hamley, I. W., Nanostructure fabrication using block copolymers. Nanotechnology 2003, 14, (10), R39-R54.

75. Kataoka, K.; Harada, A.; Nagasaki, Y., Block copolymer micelles for drug delivery: Design, characterization and biological significance. Advanced Drug Delivery Reviews 2001, 47, (1), 113131.

76. Napoli, A.; Valentini, M.; Tirelli, N.; Muller, M.; Hubbell, J. A., Oxidation-responsive polymeric vesicles. Nature Materials 2004, 3, (3), 183-189.

77. Caliceti, P.; Veronese, F. M., Pharmacokinetic and biodistribution properties of poly(ethylene glycol)-protein conjugates. Advanced Drug Delivery Reviews 2003, 55, (10), 1261-1277.

78. Golander, C. G.; Herron, J. N.; Lim, K.; Claesson, P.; Andrade, J. D., Polyethylene glycol chemistry, biotechnical and biomedical applications. Plenum Press: New York, 1992.

79. Li, S. L.; Byrne, B.; Welsh, J.; Palmer, A. F., Self-assembled poly(butadiene)-b-poly(ethylene oxide) polymersomes as paclitaxel carriers. Biotechnology Progress 2007, 23, 278-285.

80. Torchilin, V. P., Multifunctional nanocarriers. Advanced Drug Delivery Reviews 2006, 58, (14), $1532-1555$.

81. Mueller, W.; Koynov, K.; Fischer, K.; Hartmann, S.; Pierrat, S.; Basche, T.; Maskos, M., Hydrophobic shell loading of PB-b-PEO vesicles. Macromolecules 2009, 42, (1), 357-361.

82. Meng, F.; Engbers, G. H. M.; Feijen, J., Biodegradable polymersomes as a basis for artificial cells: Encapsulation, release and targeting. Journal of Controlled Release 2005, 101, (1-3), 187-198.

83. Cornelissen, J. J. L. M.; Fischer, M.; Sommerdijk, N. A. J. M.; Nolte, R. J. M., Helical superstructures from charged poly(styrene)-poly(isocyanodipeptide) block copolymers. Science 1998, 280, (5368), 1427-1430.

84. Meier, W.; Nardin, C.; Winterhalter, M., Reconstitution of channel proteins in (polymerized) ABA triblock copolymer membranes. Angewandte Chemie, International Edition 2000, 39, (24), 45994602.

85. Mueller, P.; Rudin, D. O.; Tien, H. T.; Wescott, W. C., Reconstitution of cell membrane structure in vitro and its transformation into an excitable system. Nature 1962, 194, (4832), 979-980. 
86. Mueller, P.; Rudin, D. O.; Tien, H. T.; Wescott, W. C., Methods for the formation of single bimolecular lipid membranes in aqueous solution. The Journal of Physical Chemistry 1963, 67, (2), 534-535.

87. Dani, J. A.; Levitt, D. G., Binding constants of $\mathrm{Li}+, \mathrm{K}+$, and $\mathrm{Tl}+$ in the gramicidin channel determined from water permeability measurements. Biophysical Journal 1981, 35, (2), 485-499.

88. Gómez-Lagunas, F.; Peña, A.; Liévano, A.; Darszon, A., Incorporation of ionic channels from yeast plasma membranes into black lipid membranes. Biophysical Journal 1989, 56, (1), 115-119.

89. Van Gelder, P.; Dumas, F.; Winterhalter, M., Understanding the function of bacterial outer membrane channels by reconstitution into black lipid membranes. Biophysical Chemistry 2000, 85, (2-3), 153-167.

90. Bezrukov, S. M.; Vodyanoy, I., Probing alamethicin channels with water-soluble polymers. Effect on conductance of channel states. Biophysical Journal 1993, 64, (1), 16-25.

91. Heitz, B. A.; Xu, J. H.; Jones, I. W.; Keogh, J. P.; Comi, T. J.; Hall, H. K.; Aspinwall, C. A.; Saavedra, S. S., Polymerized planar suspended lipid bilayers for single ion channel recordings: Comparison of several dienoyl lipids. Langmuir 2011, 27, (5), 1882-1890.

92. Castellana, E. T.; Cremer, P. S., Solid supported lipid bilayers: From biophysical studies to sensor design. Surface Science Reports 2006, 61, (10), 429-444.

93. Tamm, L. K.; McConnell, H. M., Supported phospholipid bilayers. Biophysical Journal 1985, 47 , (1), 105-113.

94. Sackmann, E., Supported membranes: Scientific and practical applications. Science 1996, 271, (5245), 43-48.

95. Ma, G.; Barlow, D. J.; Hollinshead, C. M.; Harvey, R. D.; Webster, J. R. P.; Lawrence, M. J., Effects of surface pressure on the structure of the monolayer formed at the air/water interface by a non-ionic surfactant. Journal of Colloid and Interface Science 2008, 317, (1), 314-325.

96. Brian, A. A.; McConnell, H. M., Allogeneic stimulation of cytotoxic t cells by supported planar membranes. Proceedings of the National Academy of Sciences 1984, 81, (19), 6159-6163.

97. Bayerl, T. M.; Bloom, M., Physical properties of single phospholipid bilayers adsorbed to micro glass beads. A new vesicular model system studied by ${ }^{2} \mathrm{H}$-nuclear magnetic resonance. Biophysical Journal 1990, 58, (2), 357-362.

98. Terrettaz, S.; Stora, T.; Duschl, C.; Vogel, H., Protein-binding to supported lipid-membranes investigation of the cholera-toxin ganglioside interaction by simultaneous impedance spectroscopy and surface-plasmon resonance. Langmuir 1993, 9, (5), 1361-1369.

99. Tatulian, S. A.; Hinterdorfer, P.; Baber, G.; Tamm, L. K., Influenza hemagglutinin assumes a tilted conformation during membrane-fusion as determined by attenuated total-reflection FTIR spectroscopy. EMBO Journal 1995, 14, (22), 5514-5523.

100. Keller, C. A.; Kasemo, B., Surface specific kinetics of lipid vesicle adsorption measured with a quartz crystal microbalance. Biophysical Journal 1998, 75, (3), 1397-1402.

101. Johnson, S. J.; Bayerl, T. M.; McDermott, D. C.; Adam, G. W.; Rennie, A. R.; Thomas, R. K.; Sackmann, E., Structure of an adsorbed dimyristoylphosphatidylcholine bilayer measured with specular reflection of neutrons. Biophysical Journal 1991, 59, (2), 289-294. 
102. Majewski, J.; Wong, J. Y.; Park, C. K.; Seitz, M.; Israelachvili, J. N.; Smith, G. S., Structural studies of polymer-cushioned lipid bilayers. Biophysical Journal 1998, 75, (5), 2363-2367.

103. Junghans, A.; Köper, I., Structural analysis of tethered bilayer lipid membranes. Langmuir 2010, 26, (13), 11035-11040.

104. Knoll, W.; Bender, K.; Forch, R.; Frank, C.; Gotz, H.; Heibel, C.; Jenkins, T.; Jonas, U.; Kibrom, A.; Kugler, R.; Naumann, C.; Naumann, R.; Reisinger, A.; Ruhe, J.; Schiller, S.; Sinner, E. K., Polymer-tethered bimolecular lipid membranes. In Polymer membranes/biomembranes, SpringerVerlag Berlin: Berlin, 2010; Vol. 224, pp 87-111.

105. Hennesthal, C.; Steinem, C., Pore-spanning lipid bilayers visualized by scanning force microscopy. Journal of the American Chemical Society 2000, 122, (33), 8085-8086.

106. Steltenkamp, S.; Muller, M. M.; Deserno, M.; Hennesthal, C.; Steinem, C.; Janshoff, A., Mechanical properties of pore-spanning lipid bilayers probed by atomic force microscopy. Biophysical Journal 2006, 91, (1), 217-226.

107. Nussio, M. R.; Oncins, G.; Ridelis, I.; Szili, E.; Shapter, J. G.; Sanz, F.; Voelcker, N. H., Nanomechanical characterization of phospholipid bilayer islands on flat and porous substrates: A force spectroscopy study. Journal of Physical Chemistry B 2009, 113, (30), 10339-10347.

108. Im, H.; Wittenberg, N. J.; Lesuffleur, A.; Lindquist, N. C.; Oh, S. H., Membrane protein biosensing with plasmonic nanopore arrays and pore-spanning lipid membranes. Chemical Science 2010, 1, (6), 688-696.

109. Weiskopf, D.; Schmitt, E. K.; Kluhr, M. H.; Dertinger, S. K.; Steinem, C., Micro-BLMs on highly ordered porous silicon substrates: Rupture process and lateral mobility. Langmuir 2007, 23, (18), 9134-9139.

110. Lazzara, T. D.; Kliesch, T. T.; Janshoff, A.; Steinem, C., Orthogonal functionalization of nanoporous substrates: Control of 3D surface functionality. ACS Appl. Mater. Interfaces 2011, In Press.

111. Dubois, L. H.; Nuzzo, R. G., Synthesis, structure, and properties of model organic-surfaces. Annual Review of Physical Chemistry 1992, 43, 437-463.

112. Laibinis, P. E.; Whitesides, G. M.; Allara, D. L.; Tao, Y. T.; Parikh, A. N.; Nuzzo, R. G., Comparison of the structures and wetting properties of self-assembled monolayers of normalalkanethiols on the coinage metal-surfaces, $\mathrm{Cu}, \mathrm{Ag}$, Au. Journal of the American Chemical Society 1991, 113, (19), 7152-7167.

113. Schwartz, D. K., Mechanisms and kinetics of self-assembled monolayer formation. Annual Review of Physical Chemistry 2001, 52, 107-137.

114. Love, J. C.; Estroff, L. A.; Kriebel, J. K.; Nuzzo, R. G.; Whitesides, G. M., Self-assembled monolayers of thiolates on metals as a form of nanotechnology. Chemical Reviews 2005, 105, (4), 1103-1169.

115. Schmitt, E. K.; Nurnabi, M.; Bushby, R. J.; Steinem, C., Electrically insulating pore-suspending membranes on highly ordered porous alumina obtained from vesicle spreading. Soft Matter 2008, 4, (2), 250-253. 
116. Romer, W.; Lam, Y. H.; Fischer, D.; Watts, A.; Fischer, W. B.; Goring, P.; Wehrspohn, R. B.; Gosele, U.; Steinem, C., Channel activity of a viral transmembrane peptide in micro-BLMs: Vpu(1-32) from HIV-1. Journal of the American Chemical Society 2004, 126, (49), 16267-16274.

117. Drexler, J.; Steinem, C., Pore-suspending lipid bilayers on porous alumina investigated by electrical impedance spectroscopy. The Journal of Physical Chemistry B 2003, 107, (40), 1124511254.

118. Römer, W.; Steinem, C., Impedance analysis and single-channel recordings on nano-black lipid membranes based on porous alumina. Biophysical Journal 2004, 86, (2), 955-965.

119. Jonsson, P.; Jonsson, M. P.; Hook, F., Sealing of submicrometer wells by a shear-driven lipid bilayer. Nano Letters 2010, 10, (5), 1900-1906.

120. Tabor, D.; Winterton, R. H. S., The direct measurement of normal and retarded van der Waals forces. Proceedings of the Royal Society of London. A. Mathematical and Physical Sciences 1969, 312, (1511), 435-450.

121. Israelachvili, J. N., The calculation of van der Waals dispersion forces between macroscopic bodies. Proceedings of the Royal Society of London. A. Mathematical and Physical Sciences 1972, $331,(1584), 39-55$.

122. Israelachvili, J., Solvation forces and liquid structure, as probed by direct force measurements. Accounts of Chemical Research 1987, 20, (11), 415-421.

123. Claesson, P. M.; Ederth, T.; Bergeron, V.; Rutland, M. W., Techniques for measuring surface forces. Advances in Colloid and Interface Science 1996, 67, 119-183.

124. Binnig, G.; Quate, C. F.; Gerber, C., Atomic force microscope. Physical Review Letters 1986, 56, (9), 930-933. 


\section{EXPERIMENTAL}

\subsection{Methods}

Microscopy is the study of specimens which cannot be observed by eye due to their size being smaller than the resolution of the human eye. The main branches of microscopy are optical, electron and scanning probe microscopies. In optical microscopy, electromagnetic radiation interacts with the sample, in electron microscopy an electron beam is used to study the sample and during scanning probe microscopy a sharp tip probes the surface. Optical and scanning probe microscopies were used extensively in this work and the details of these techniques are discussed in the following text.

\subsubsection{OPTICAL MICROSCOPY: WHITE LIGHT AND FLUORESCENCE MICROSCOPY}

In a traditional epi-fluorescence microscope, the sample is illuminated by excitation light (mercury arc discharge lamp) which is passed through an excitation filter so that only the light of a defined wavelength passes through and interacts with the fluorophores in the sample. After passing the filter, the light is directed towards the sample by a dichromatic mirror. Following the interaction with the sample, the emission light passes through a dichromatic mirror and is directed towards an emission filter and then, the eyepiece. 

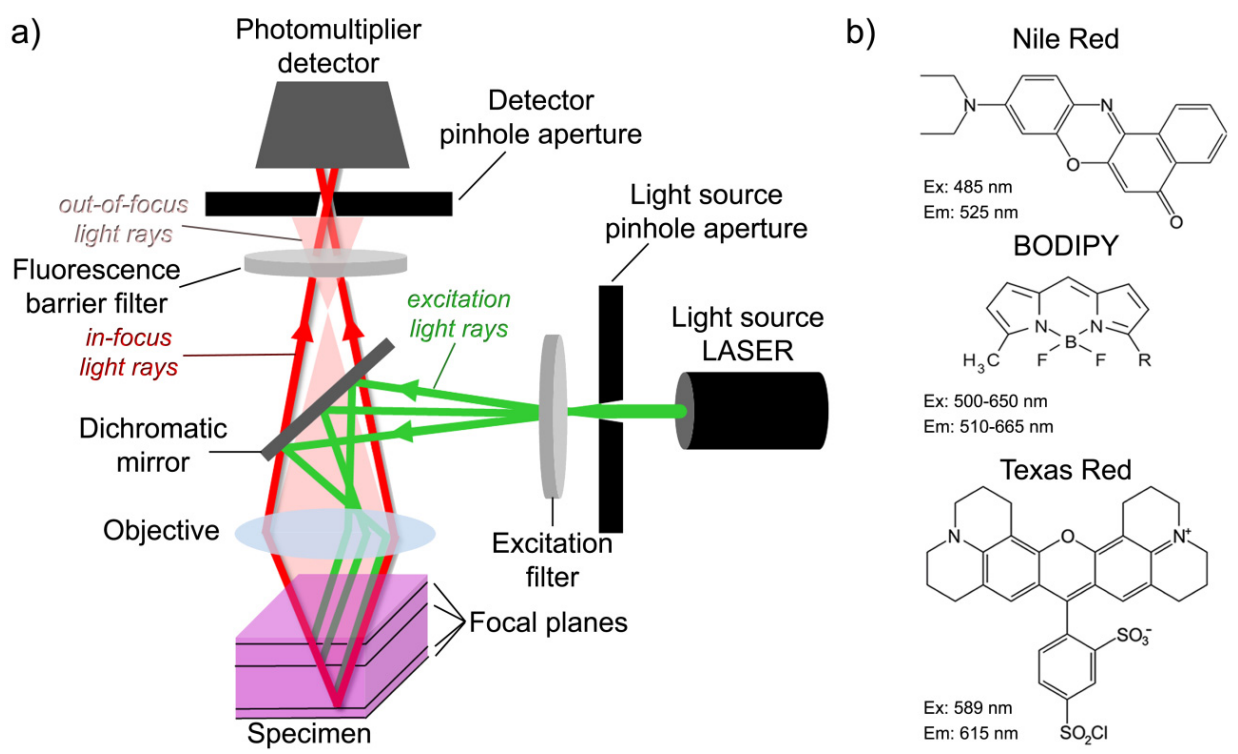

Figure 2.1 (a) Schematic setup of a confocal fluorescent microscope. (b) Chemical structures of dye molecules which can undergo excitation and emission to create fluorescence.

Confocal laser scanning microscopy (CLSM) is a variation of epi-fluorescence microscopy where a pinhole placed between the excitation source (laser) and the sample reduces the size of the light beam interacting with the sample (Figure 2.1a). ${ }^{1}$ Once the light has interacted with the fluorescent dyes (Figure 2.1b) within the sample, it is directed towards a detector in front of which another pinhole aperture is reducing the amount of light coming from out-of-focus planes. In confocal microscopy, resolution is improved only slightly, however, the exclusion of secondary fluorescence coming from areas outside of the focal plane significantly improves the image quality. Another difference between a traditional microscope and a confocal microscope is that the sample is raster scanned by a focused light beam. CLSM can also be used to obtain 3D images of the sample by acquiring consecutive images of the sample in the z-axis at different focal planes (z-stacks). These thin $(0.5-1 \mu \mathrm{m})$ optical sections are collected and 3D images can be reconstructed.

In addition to visualization of fluorescently labeled samples, fluorescent recovery after photobleaching (FRAP) experiments can be performed with CLSM in order to measure two dimensional diffusion of labeled phospholipids or proteins. FRAP experiment is carried out by photobleaching a defined, usually circular area of the sample by short (2-3 s) high intensity illumination pulses. The photochemical oxidation of dye molecules results in a dark spot on the sample. Fluorescence recovery of the photobleached area is 
recorded at the same time as the fluorescence intensity of a reference area. The recovery of fluorescence is only possible if fluorophores from non-bleached areas of the sample can diffuse into the bleached region. Fluorescence recovery curve is obtained by plotting normalized fluorescence intensity of the region of interest as a function of time (Figure 2.2).
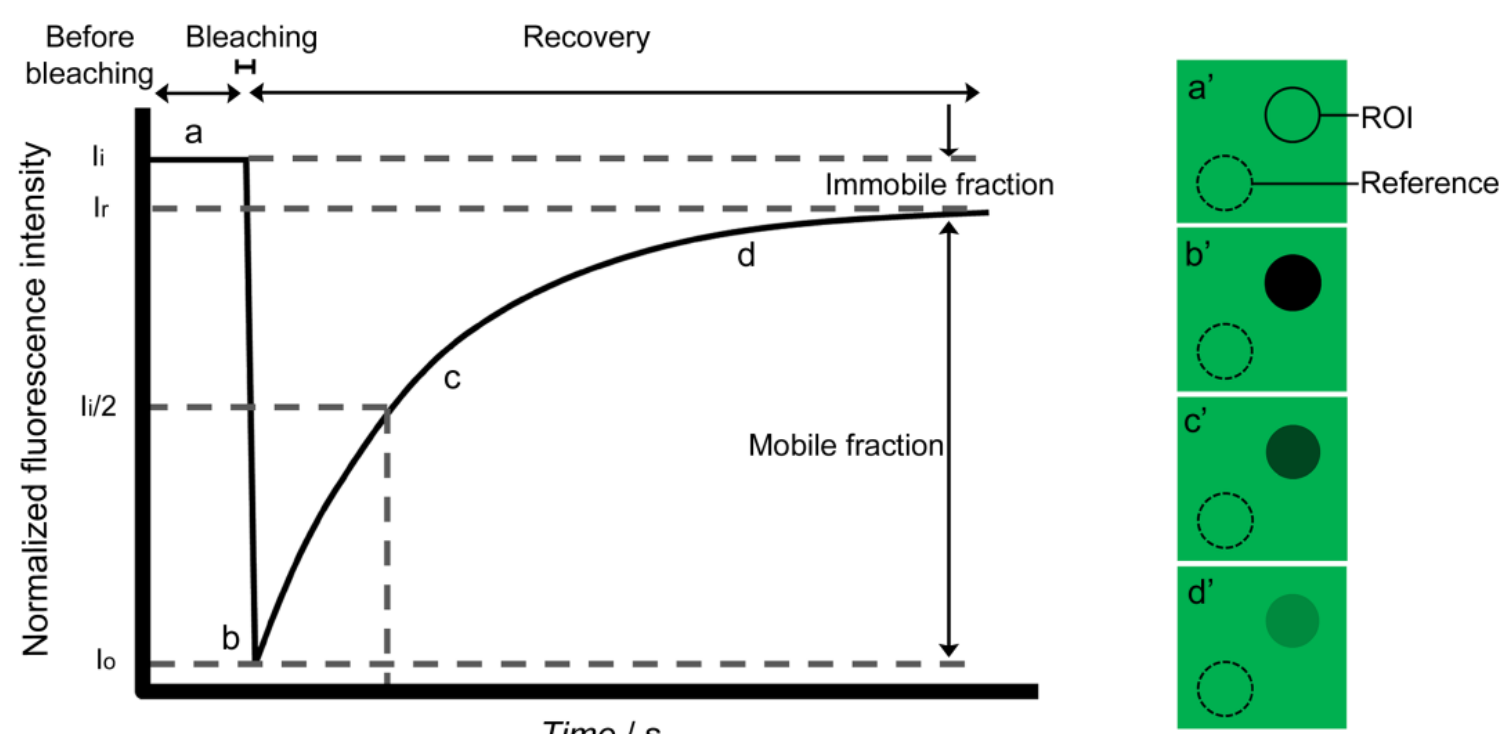

Figure 2.2. Schematic representation of a FRAP experiment showing fluorescence intensity as a function of time. The initial fluorescence intensity $\left(\mathrm{I}_{\mathrm{i}}\right)$ of a region of interest (ROI) is shown in the graph as $a$ and as $a$, in the drawing of the sample. As ROI is photobleached ( $b$ in the graph and $b$ ' in the drawing), the fluorescence intensity drops to zero $\left(\mathrm{I}_{0}\right)$. When diffusion is possible, fluorescence intensity recovery takes place (c, $d$ in the graph and c', $d$ ' in the drawing) and ROI regains almost all of its intensity $\left(\mathrm{I}_{\mathrm{r}}\right)$.

Assuming a Gaussian profile of the bleached area, diffusion coefficient $D$ can be calculated from the radius $r$ of the bleached spot and the time $t_{1 / 2}$ needed for the bleached area to recover half of its initial fluorescence intensity:

$$
D=\frac{r^{2}}{4 t_{1 / 2}}
$$

Due to the non-continuous fluorescence of the membrane patch on the porous surface (due to fluorescence quenching on the gold-covered pore rims), the Gauss radius of the bleached area on the porous substrate was determined by fitting an intensity profile using a MATLAB fitting procedure (see appendix, Figure A2.1).

Lipid diffusion coefficient was obtained using the theory of Axelrod et al. where equation 2.2 was fitted to the experimentally obtained recovery curves: 


$$
F_{\mathrm{K}}(t)=\left(Q P_{0} C_{0} / A_{\mathrm{f}}\right) \sum_{n=0}^{\infty}\left((-K)^{n} / n !\right)\left(\frac{1}{1+n\left(1+2 t / \tau_{\mathrm{D}}\right)}\right)
$$

The fluorescence at time $t\left(F_{\mathrm{K}}(t)\right)$ is valid for a Gaussian intensity profile with $\tau_{\mathrm{D}}=\omega^{2} / 4 D$. $D$ is the lateral diffusion constant of the lipid, $Q$ is the product of all quantum efficiencies of light absorption, emission and detection, $A_{\mathrm{f}}$ is the attenuation factor of the beam during observation of recovery, $P_{0}$ the total laser power, $\omega$ is the half-width at $\mathrm{e}^{-2}, C_{0}$ is the initial fluorophore concentration, and $K$ is the bleaching induced at time $T(K=\alpha \operatorname{TI}(0))$, $\alpha T I(0)$ is the rate of bleaching in the center. ${ }^{2}$

\subsubsection{SCANNING PROBE MICROSCOPY: ATOMIC FORCE MICROSCOPY (AFM)}

Scanning probe microscopy is based on the interaction of a sharp tip (probe) with the sample where the interactions are monitored by deflection of a soft cantilever on which the tip is mounted (Figure 2.3). ${ }^{3}$ Microfabicated from silicon or silicon nitride, AFM cantilevers are fixed on the side of a chip (Figure 2.3a). The sharp tip which interacts with the surface is mounted at the end of the cantilever (Figure 2.3b-c) and it is its size which limits the resolution during imaging. ${ }^{4}$ Commercially available AFM probes have a tip radii ranging between $5 \mathrm{~nm}$ and $50 \mathrm{~nm}$ (Figure 2.3d, inset).
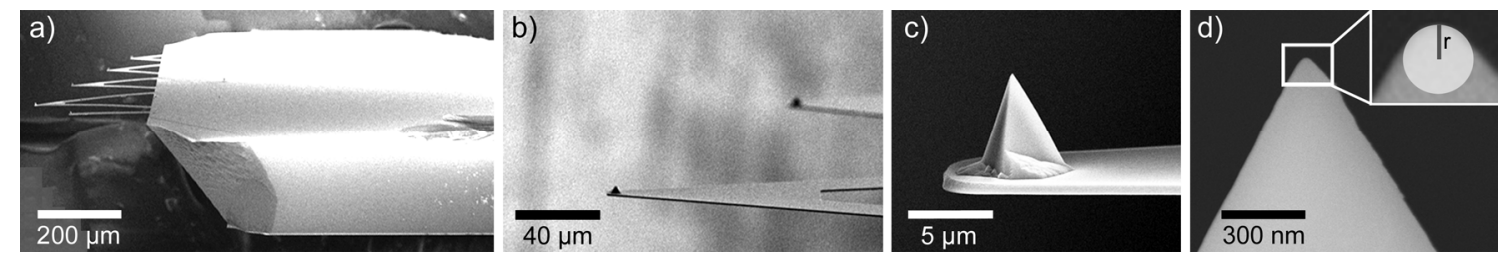

Figure 2.3 (a) AFM probe, (b) cantilevers, (c) sharp AFM tip mounted at the end of a cantilever, (d) a close-up view of the AFM tip with approximately $50 \mathrm{~nm}$ tip radius $r$ (shown in the inset).

During AFM imaging, topographic images of the surface are obtained by raster scanning the sample. Two imaging modes are available and differ in the way the tip is moved across the surface. When imaging in contact mode, the tip is continuously in contact with the surface with a predetermined force (Figure 2.4b, top). This is achieved 
by a feedback mechanism, where a given cantilever deflection setpoint is kept constant by moving the tip to and away from the surface by piezo actuators.

a)

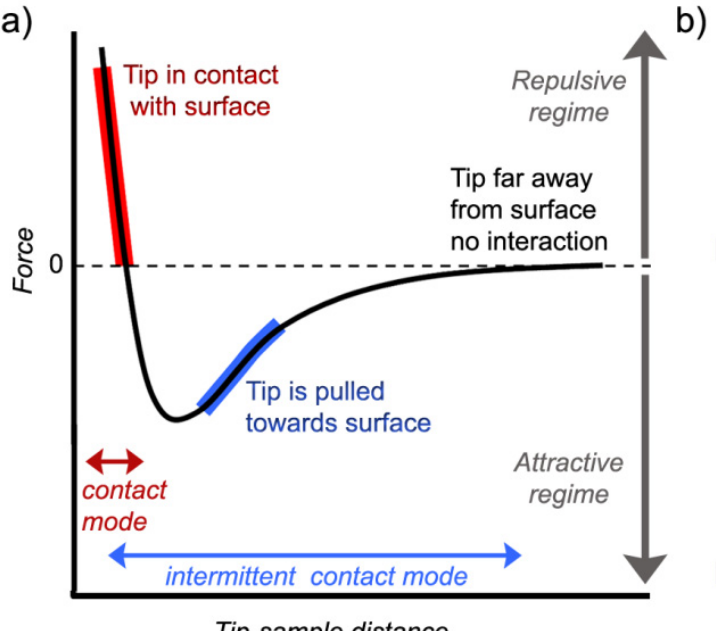

b)

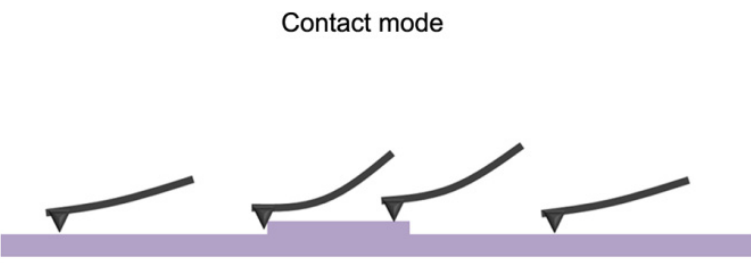

Intermittent contact mode

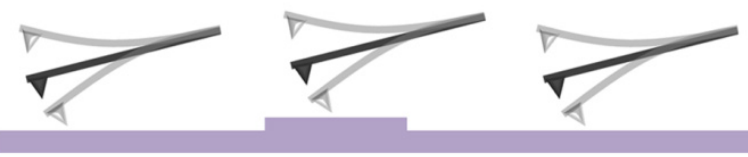

Figure 2.4 (a) Energy potential diagram and (b) AFM imaging modes: contact mode (top) and intermittent contact mode (bottom).

During intermittent contact mode (also called tapping mode), imaging is achieved by oscillating the cantilever close to its resonance frequency as it is raster scanning the surface (Figure 2.4b, bottom). The feedback system maintains constant oscillation amplitude of the cantilever by adjusting the distance between the tip and the sample. The advantage of contact mode imaging is the control of the maximal normal force of the tip on the sample, however lateral stresses during imaging can be damaging to soft samples. ${ }^{5}$ The limited contact time of the tip with the surface during intermittent contact mode imaging reduces the force exerted on the sample. AFM measurements can be performed in air, liquid or vacuum which makes the technique versatile.

Force versus distance experiments are another type of experiment which can be performed with the AFM and provide information about the forces acting between the tip and the surface. The sharp tip is moved in the z-axis until it reaches the surface, it can be pushed into the surface, paused or immediately retracted to its initial position far away from the surface. The forces acting between the surface and the AFM tip are monitored by the deflection of the cantilever, which is determined by the optical lever method that 
magnifies small displacement during imaging. ${ }^{6,7}$ Force-distance curves are obtained by plotting cantilever deflection signal versus piezo displacement.

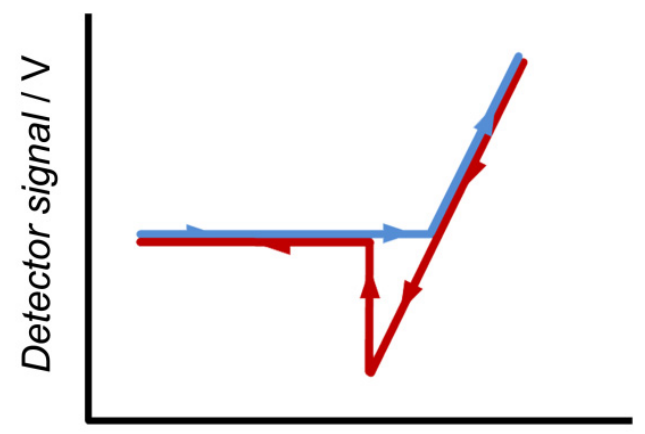

Piezo position / nm

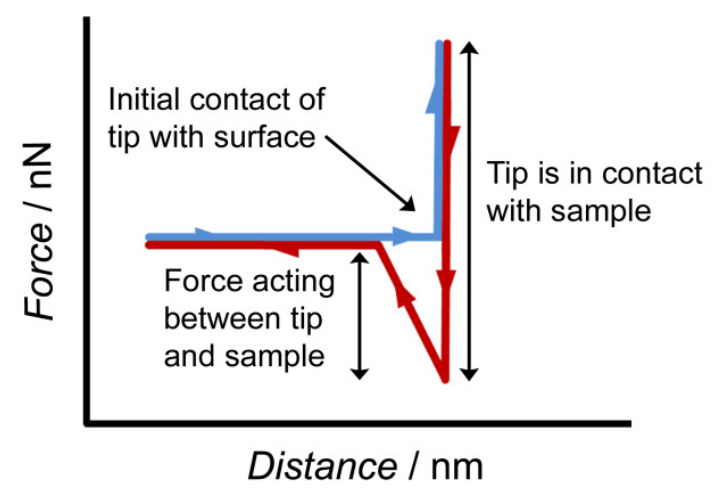

Figure 2.5 Schematic representation of a force curve performed on a hard surface. On the left, data is presented in its raw form: detector signal versus piezo position. After determining the sensitivity of the detector and calculating the spring constant of the cantilever used to obtain the force curve, a force versus distance curve is determined.

Cantilever deflection $d_{\mathrm{c}}$ is then converted into a force $F$ by multiplying it with the spring constant of the cantilever $k_{\mathrm{c}}$ :

$$
F=k_{\mathrm{C}} d_{\mathrm{c}}
$$

Manufacturers of AFM probes usually provide an approximate range of the cantilever spring constant based on its physical properties (size, length, composition, elastic modulus of material), however, spring constant calibration procedure is usually performed before each experiment. The method used throughout this work is the thermal noise method integrated in software of the instrument. This calibration method is based on the equipartition theorem which states that the mean thermal energy of any harmonic system at temperature $T$ is equal to $k_{\mathrm{B}} T / 2$ per degree of freedom. Developed by Hutter and Bechhofer ${ }^{8}$ refined by Butt and Jaschke ${ }^{9}$, it is suggested that when a cantilever is modeled as a harmonic oscillator, the mean square deflection $\left\langle z^{2}\right\rangle$ of the cantilever due to thermal fluctuations can be approximated by equation 2.3 : 


$$
\left\langle z^{2}\right\rangle=\frac{k_{\mathrm{B}} T}{k_{\mathrm{C}}}
$$

where $k_{\mathrm{c}}$ is the spring constant of the cantilever.

Experimentally, the sensitivity of the cantilever is obtained by performing a forcedistance curve on a hard surface (glass, silicon) and thermal fluctuations of the cantilever are acquired by recording the amplitude of cantilever deflection while it is in air or liquid while being far away from the surface. The so obtained spectrum shows a peak at the cantilever's resonance frequency, which corresponds to the first vibration mode. The peak is fitted with a Lorentzian curve and the mean square deflection is obtained by integration of that area. Correction factor $\mathrm{B}=0.764$ needs to be included to correct for the error associated with the optical lever method used to obtain the cantilever deflection. ${ }^{10,11}$ 


\subsection{MATERIALS AND METHODS: BLOCK COPOLYMER MEMBRANES}

\subsubsection{MATERIALS}

Polymersomes used in this work were generously donated by W. Müller and M. Maskos (University Mainz, Mainz, Germany). Vesicle solutions were produced in purified water and tetrahydrofuran (THF, uvasol grade, Riedel-de Haen, destabilized by distillation). Nile Red fluorescent dye (Figure 2.1b) was used without purification (99\%, Acros). Linear poly(butadiene) was purchased from Aldrich with $M_{\mathrm{n}}=1.53-2.07 \mathrm{~kg} / \mathrm{mol}$. The synthesis of poly(butadiene)- $b$-poly(ethylene oxide) was performed according to Maskos et $a l .{ }^{12}$ The copolymer $\mathrm{PB}_{130}-b-\mathrm{PEO}_{66}-\mathrm{COOH}$ was characterized by MALDI-TOF mass spectrometry, resulting in $M_{\mathrm{n}}=11 \mathrm{~kg} / \mathrm{mol}, M_{\mathrm{w}} / M_{\mathrm{n}}=1.05$, and $29 \% \mathrm{w} / \mathrm{w}$ PEO (the indices represent the number-average degree of polymerization of the individual blocks as determined by ${ }^{1} \mathrm{H}$ NMR). ${ }^{13}$ Water used during experiments was filtered by a Millipore system (MilliQ System from Millipore, Molsheim, France; resistance $>18 \mathrm{M} \Omega \mathrm{cm}^{-1}$ ). Porous silicon nitride substrates (Figure 2.6) used in this work were purchased from fluXXion B.V. (Eindhoven, NL).

\subsubsection{SUBSTRATE PREPARATION}

The porous substrates bought from fluXXion B.V. are manufactured by deposition of a silicon nitride $800 \mathrm{~nm}$ thick porous films on a silicon support. ${ }^{14}$ The porous areas on the 5 mm square substrates are arranged in a pattern of 14 lines with 10 rectangles per each line (Figure 2.6b). This particular arrangement together with the writing "FLUXX SIEVE 1.20 " serve as a Cartesian coordinate system for localization of membrane patches during experiments. The porous area closest to the letter $F$ is defined as position $(0,0)$. For example, the patch in figure $2.6 \mathrm{~b}$ is located in the $3^{\text {rd }}$ row, and in the $4^{\text {th }}$ column, therefore its location is $(3,4)$. 
a)

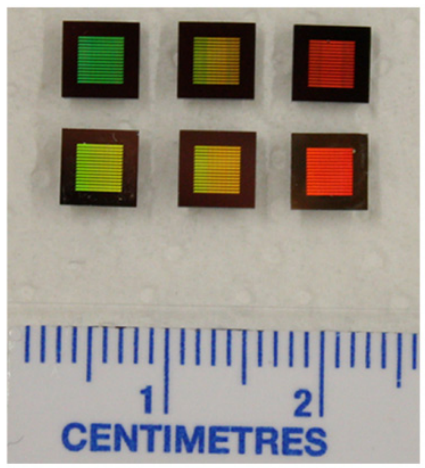

d)

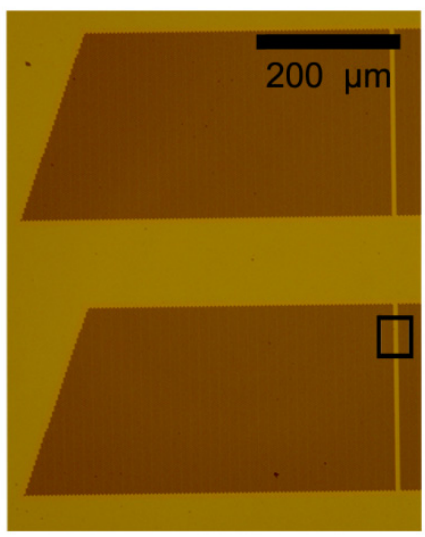

b)

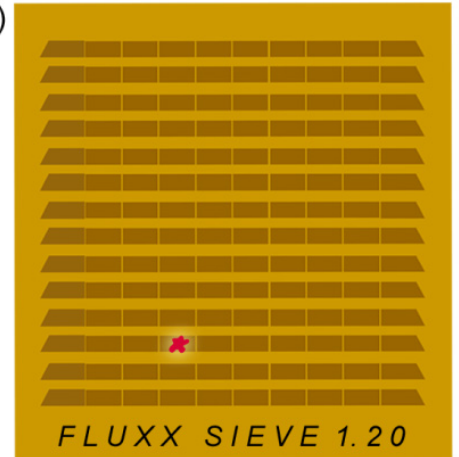

e)

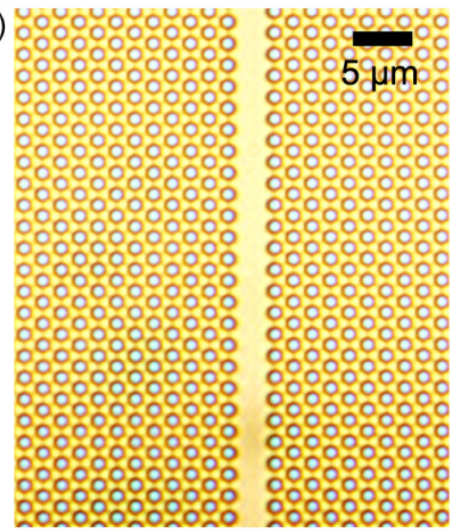

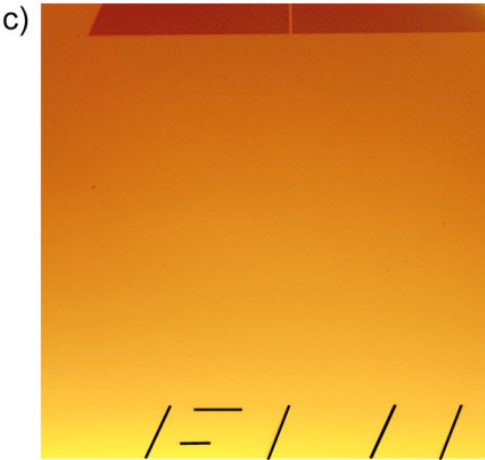

f)

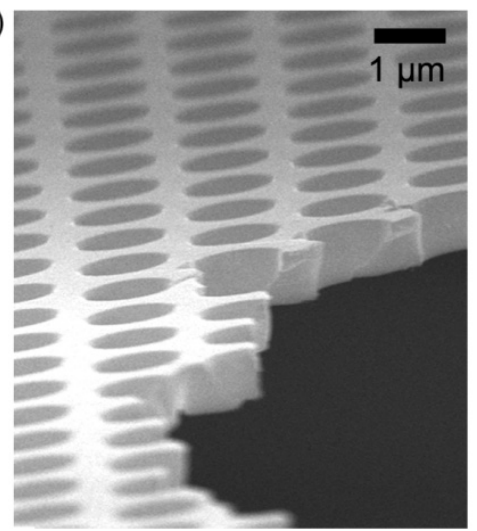

Figure 2.6 (a) A photograph of six, square, fluxxion substrates where the colored area corresponds to pores. (b) Schematic representation of 14 lines of 10 porous areas found in each substrate. (c) Light microscope image of the first porous area and of the FLUXX SIEVE 1.20 writing which serves as a Cartesian coordinate of $(0,0)$. (d) Close-up of the flat (brighter) and porous (darker) areas on the substrate. (e) Close-up of porous area shown in $d$, (f) SEM image of the silicon nitride porous film.

Prior to use, the substrates were treated with argon plasma for $30 \mathrm{~s}$ (Plasma Cleaner, Harrick Plasma, Ithaca, NY).

\subsubsection{PORE-SPANNING POLYMER MEMBRANE PREPARATION}

Poly(butadiene)- $b$-poly(ethylene-oxide) (PB-PEO) polymersomes were prepared by rehydration method. Starting from a Nile Red dye/copolymer solution in THF, a thin film was created and dried at $50^{\circ} \mathrm{C}$ under vacuum. $1 \mathrm{M} \mathrm{NaCl}$ solution was added and the film rehydration process was supported by ultrasound use, resulting in polymersomes containing $1 \mathrm{M} \mathrm{NaCl}$ solution in their interior.

To prepare polymer membranes on the porous surfaces, vesicle solution was added to a freshly cleaned porous substrate, which was immobilized in a home made substrate holder, and incubated for 5 min during which the vesicles settled down on the porous substrate. Purified water was then added and the chamber left undisturbed 
for 20 - 60 minutes to allow the vesicles to spread by osmotic pressure change. As the vesicles swelled and burst, the polymersome membranes covered some areas of the porous substrate.

\subsubsection{FLUORESCENCE}

An upright optical microscope (Olympus BX-51, Olympus Germany GmbH, Hamburg, Germany) equipped with a filter for Nile Red fluorescence (UMNG2, Olympus Germany GmbH, Hamburg, Germany) and water immersion objectives with $40 \times$ (Olympus, LUMPlanF1 40XW, N.A. $=0.8)$, and $100 \times($ Olympus, LUMPlanF1 $100 \mathrm{~W}$, N.A. $=1.00)$ magnifications and an upright confocal laser microscope (LSM 710 Axio Examiner, Carl Zeiss MicroImaging GmbH, Jena, Germany) equipped with 539-753 nm filter and water immersion objectives with 40× (Zeiss, wPlanAPOCHROMAT, N.A. $=1.0)$ and $63 \times($ Zeiss, wPlan-APOCHROMAT, N.A. $=1.0)$ magnifications were used for acquiring fluorescence images of the spread polymeric membranes.

The presence of the fluorescent Nile Red dye in the hydrophobic membrane core was used to identify and locate membrane patches formed form vesicle rupture. Once located by fluorescence imaging, white light upright optical microscope was used to determine the location of the membrane patches through the cartesian coordiante system of the porous areas on the substrate. Furtermore, confocal laser scanning microscopy (CLSM) was used to confirm that the vesicles did rupture and membranes were formed. By acquiring z-direction consecutive images of the membrane (z-stacks, $\sim 900 \mathrm{~nm}$ z-resolution), 3D images of the structures on the surface were distinguishable: spread membrane, flattended vesicles and vesicles containg smaller vesicles inside of them.

\subsubsection{ATOMIC FORCE MICROSCOPY}

Experiments were performed in a liquid environment with a MFP-3D (Asylum Research, Santa Barbara, CA) AFM equipped with top view optics. Imaging and force curves were carried out using silicon nitride AFM probes (MLCT-AU cantilever C, $310 \mu \mathrm{m}$ in length) purchased from Veeco Instruments (Mannheim, Germany) with 
spring constants between 0.01 and $0.04 \mathrm{~N} / \mathrm{m}$ with a tip radius between 10 and $30 \mathrm{~nm}$ were used for imaging and force curves acquisition. The exact spring constants of the cantilevers were determined by the thermal noise method ${ }^{9}$ integrated into the MFP3D IGOR software. Force curves were performed in the center of the membranespannig pores with varying velocities and varying maximal indentation forces.

\subsubsection{DATA ANALYSIS}

Force curves were corrected so that the initial contact of the tip with the surface, based on the deflection of the cantilever, was at zero distance and zero force. MathCad program was used to model force distance curves according to the modified Kelvin-Voight model (Figure 3.11). The values from model force distance curves best representing the actual force curves were used to calculate the mechanical constants of the membrane. Furthermore, the viscoelastic loss (energy dissipation) was obtained by calculating the area enclosed by the indentation and retraction curves. 


\subsection{MATERIALS AND METHODS: PHOSPHOLIPID MEMBRANES}

\subsubsection{MATERIALS}

1-palmitoyl-2-oleoyl-sn-glycero-3-phosphocholine (POPC), 1,2-dioleoyl-sn-glycero-3phosphocholine (DOPC), 1,2-diphytanoyl-sn-glycero-3-phosphocholine (DPhPC) were purchased from Avanti Polar Lipids (Alabaster, AL, USA). Cholesterol was purchased from Sigma-Aldrich (Steinheim, Germany). Lipids were dissolved in chloroform (Roth, Karlsruhe, Germany) and were labeled (0.1 mol \%) with either 2-(4,4-difluoro-5-methyl4-bora-3a,4a-diaza-s-indacene-3-dodecanoyl)-1-hexadecanoyl-sn-glycero-3-

phosphocholine ( $\beta$-BODIPY 500/510 C12-HPC, Invitrogen, Karlsruhe, Germany) or sulforhodamine-1,2-dihexanoyl-sn-glycero-3-phosphoethanolamine (TexasRed-DHPE, Sigma-Aldrich, Steinheim, Germany). Mercaptoethanol (Sigma-Aldrich, Steinheim, Germany), ethanol (Sigma-Aldrich, Steinheim, Germany), isopropanol (VWR, Fontenaysous-Bois, France), tetradecanethiol (Fluka, Buchs, Switzerland), sodium hydrogen phosphate dihydrate (AppliChem, Darmstadt, Germany) were all used without further purification. Water used in preparation of buffers was filtered by a Millipore system (MilliQ System from Millipore, Molsheim, France; resistance $>18 \mathrm{M} \Omega \mathrm{cm}^{-1}$ ).

\subsubsection{GIANT UNILAMELLAR VESICLE PREPARATION}

Giant unilamellar vesicles (GUVs) were prepared by electroformation. ${ }^{15,}{ }^{16}$ Briefly, 50 $\mu \mathrm{L}$ of $1 \mathrm{mg} \mathrm{mL} \mathrm{mL}^{-1}$ POPC, DOPC, DOPC/cholesterol or DPhPC lipid solution was deposited on indium tin oxide (ITO) slides and spread uniformly using a sterile needle. The ITO slides were placed under vacuum for at least 3 hours at $64^{\circ} \mathrm{C}$ to remove any residual solvent. Subsequently, two ITO slides covered with lipid films, conductive copper tape and a $1 \mathrm{~mm}$ thick square Teflon spacer between the slides, were used to create a sealed chamber (approx. $1.2 \mathrm{ml}$ volume), which was filled with $0.3 \mathrm{M}$ sucrose solution. The chamber was connected to a waveform generator and a cycle was carried out at $12 \mathrm{~Hz}$, where voltage increments were performed every $60 \mathrm{~s}$ : beginning at $0.05 \mathrm{~V}$, followed by $0.01 \mathrm{~V}$ voltage steps until $0.2 \mathrm{~V}$ was reached, and finally the voltage was increased by $0.1 \mathrm{~V}$ voltage steps until a constant $1.6 \mathrm{~V}$ was reached (total duration was 3 
hours). At the end, a square $5 \mathrm{~Hz}$ wave was applied for $5 \mathrm{~min}$. The GUVs were transferred to a plastic vial and stored at $4{ }^{\circ} \mathrm{C}$ for up to 2 weeks.

\subsubsection{SUBSTRATE PREPARATION: FUNCTIONALIZATION PROCEDURE}

Silicon nitride substrates with pore radii of $600 \mathrm{~nm}$ were purchased from fluxxion B.V. (Eindhoven, The Netherlands) (see Figure 2.6). The porous substrates were cleaned in $\mathrm{O}_{2}$ plasma to remove any contaminants and coated with a 2-3 $\mathrm{nm}$ thick layer of chromium followed by a 10-15 nm thick layer of gold (Bal-Tec MCS610 evaporator equipped with Bal-Tec QSG 100 quartz film thickness monitor). The gold-coated substrates were subsequently oxygen-plasma ( $1 \mathrm{~min})$ and argon-plasma $(1 \mathrm{~min})$ treated and placed in a 20 $\mathrm{mM}$ ethanolic mercaptoethanol (or $10 \mathrm{mM}$ ethanolic tetradecanthiol) self-assembly solution for 1 hour. The substrates were rinsed with ethanol prior to use by consecutive immersion in ethanol-filled beakers.

\subsubsection{PORE-SPANNING LIPID MEMBRANE PREPARATION}

Directly after rinsing the functionalized substrate with ethanol, it was placed in a homemade Teflon holder filled with ethanol. Ethanol was then replaced by phosphate buffered saline (PBS: $20 \mathrm{mM}$ sodium hydrogen phosphate dehydrate and $100 \mathrm{mM} \mathrm{NaCl}, \mathrm{pH} 7.4$ ) and $20 \mu 1$ of GUVs were added. GUVs migrated (due to the density difference between $0.3 \mathrm{M}$ sucrose inside the GUVs and the PBS buffer) to the porous surface and spread, creating lipid bilayer patches. The resulting patches were observed by fluorescence microscopy and their location determined in relation to the rows-and-columns pattern of the porous substrate (Figure 2.6). The DPhPC hybrid pore-spanning membranes were prepared by placing a gold-coated porous silicon substrate in $10 \mathrm{mM}$ ethanolic tetradecanethiol solution for 1 hour. The substrate was rinsed with ethanol, placed in a Teflon holder filled with ethanol, which was subsequently replaced with PBS buffer before DPhPC GUVs were added.

\subsubsection{FLUORESCENCE AND CLSM}

Fluorescence microscopy was used to locate bilayer patches on porous substrates. Due to the presence of a gold film atop the silicon nitride porous substrate which quenches 
fluorescence up to $20 \mathrm{~nm}$ away from the surface, bilayer membranes formed on flat areas of the surface are not visible. ${ }^{17}$ However, the lack of gold in the pores results in highly fluorescent membrane-covered pores. Furthermore, movies of vesicle spreading were acquired at the fluorescence microscope giving insight into the vesicles spreading mechanism. CLSM z-stack images ( $900 \mathrm{~nm}$ z-resolution) of both phospholipid vesicles and patches were collected and provided information about the single bilayer nature of the membranes covering the pores.

\subsubsection{ATOMIC FORCE MICROSCOPY}

See Section 2.2.5.

\subsubsection{DATA ANALYSIS}

Force indentation curves obtained on the pore-spanning membranes were analyzed by calculating the slope of the indentation curve $\left(k_{\text {app }}\right)$. The values were then compiled and histograms were computed. The histograms were fitted with Gaussian fits to obtain average values and those values were used to calculate membrane tension. 


\subsection{APPENDIX}

MATLAB procedure used to determine the fluorescence intensity profile of the sample and to perform a Gaussian fit to obtain the Gauss radius from FRAP experiments.
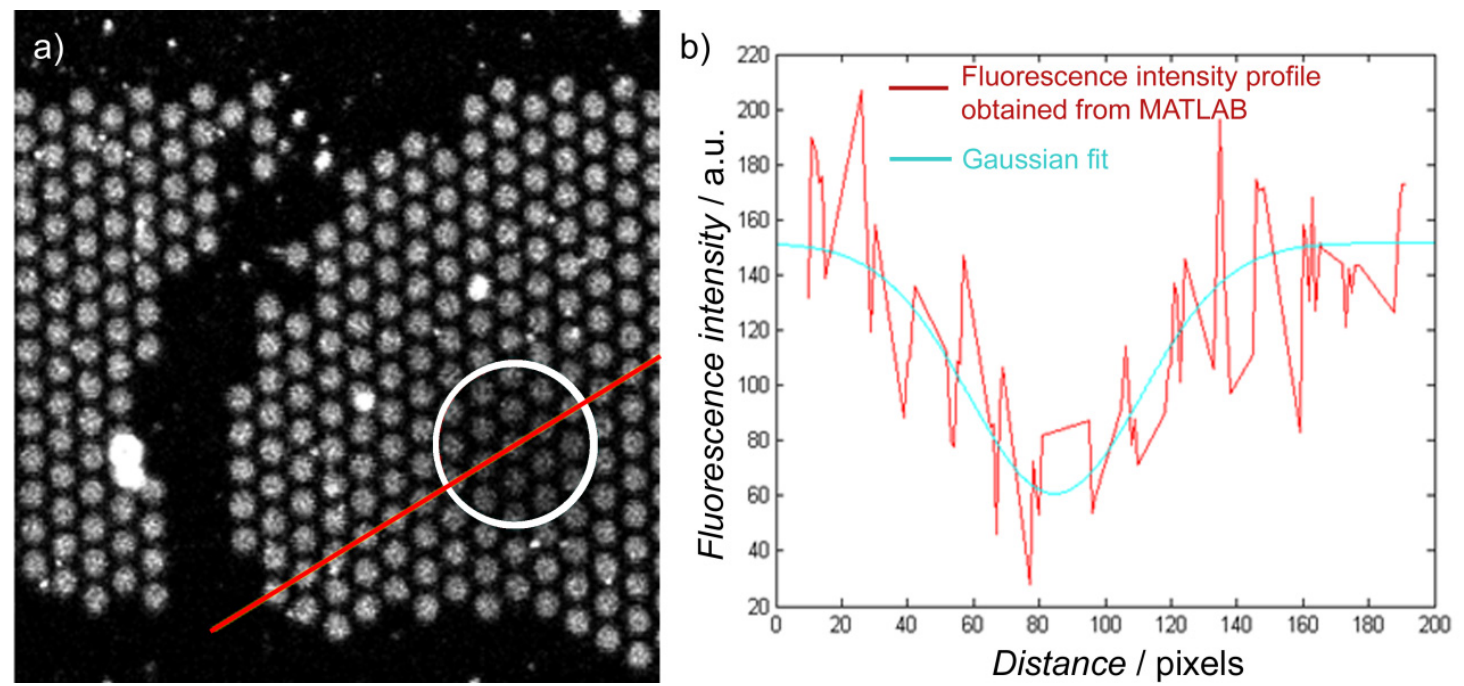

Figure A2.1 (a) Pore-spanning membrane patch where an area was photobleached (circle). (b) Fluorescence intensity profile (acquired from image shown in a) obtained from MATLAB with Gaussian fit in order to determine the Gauss radius.

MATLAB script:

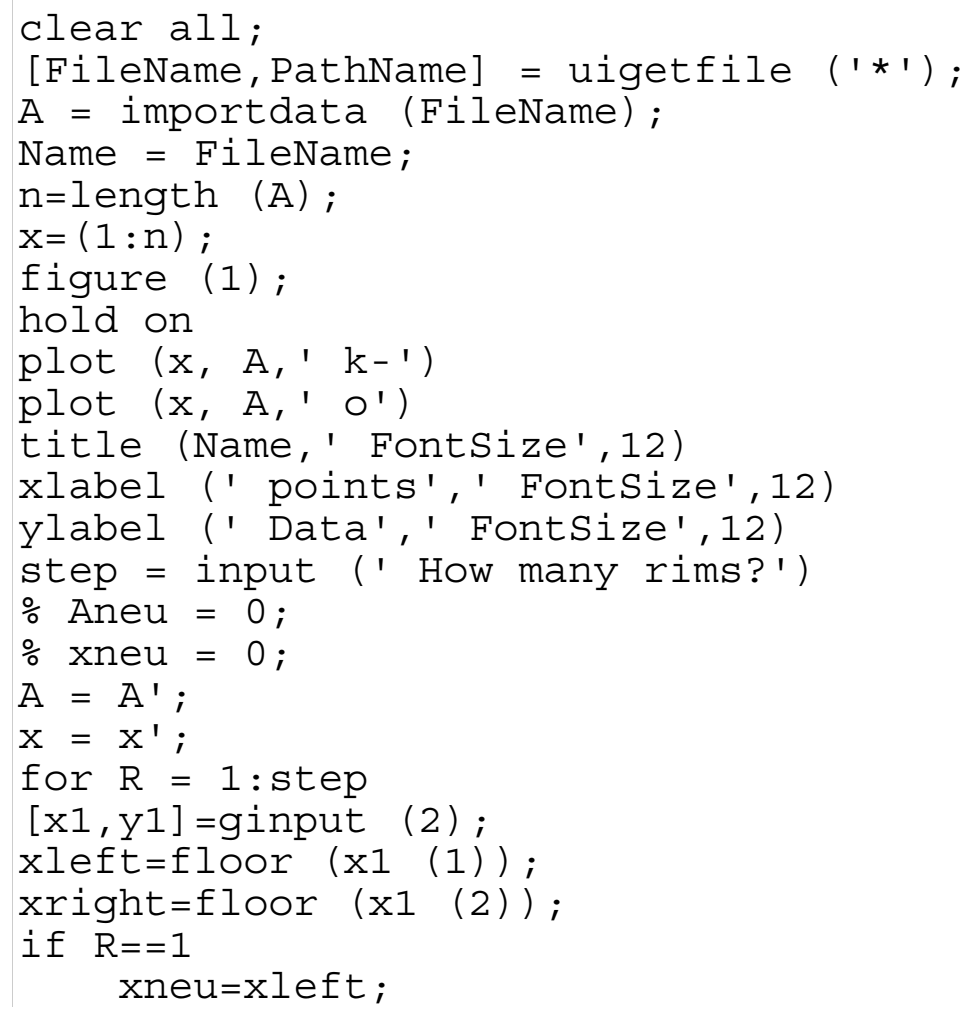




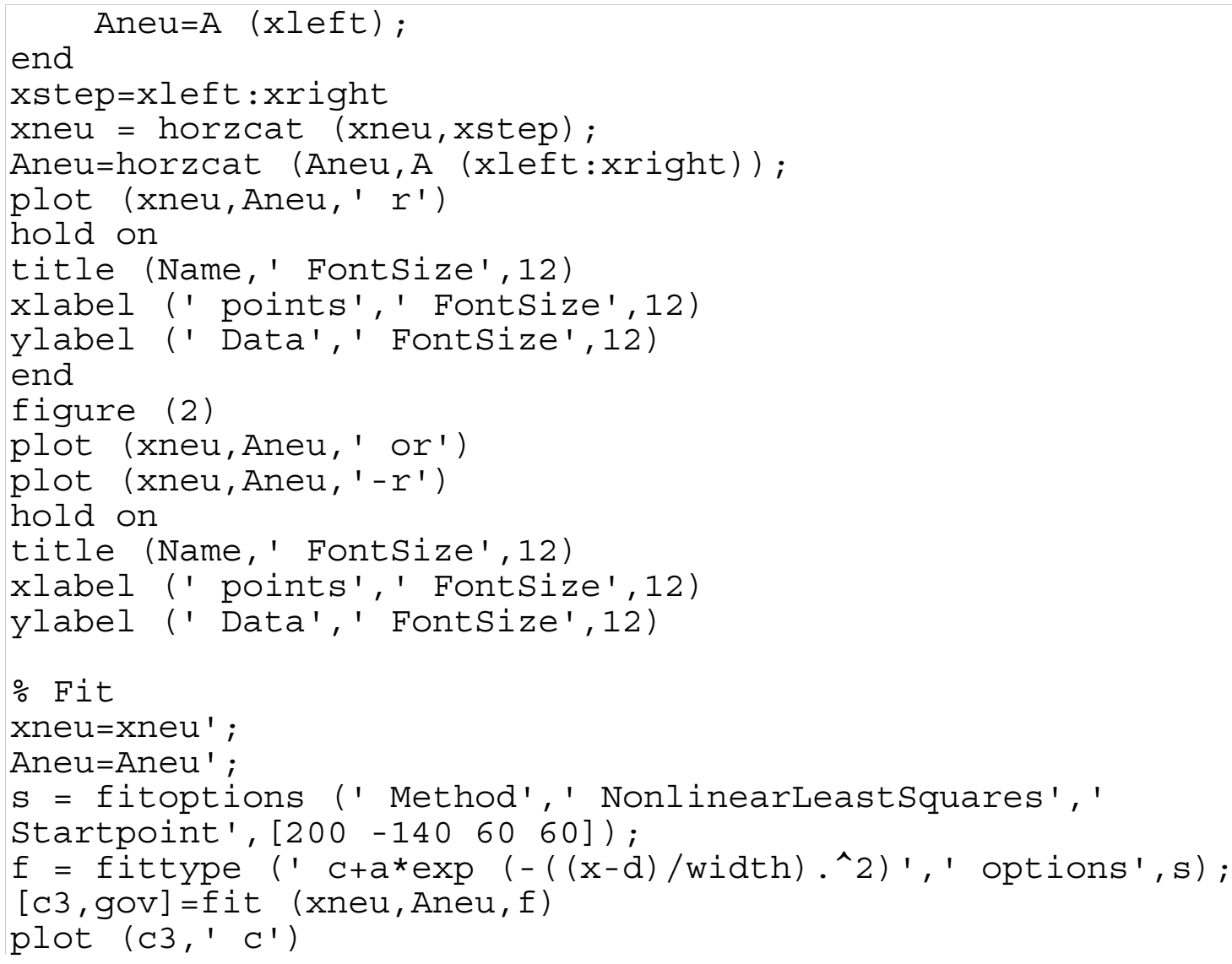




\subsection{REFERENCES}

1. Pawley, J., Handbook of biological confocal microscopy. $3^{\text {rd }}$ ed.; Springer: New York, 2006.

2. Axelrod, D.; Koppel, D. E.; Schlessinger, J.; Elson, E.; Webb, W. W., Mobility measurement by analysis of fluorescence photobleaching recovery kinetics. Biophysical Journal 1976, 16, (9), 1055-1069.

3. Radmacher, M.; Tillamnn, R. W.; Fritz, M.; Gaub, H. E., From molecules to cells: Imaging soft samples with the atomic force microscope. Science 1992, 257, (5078), 1900-1905.

4. Dai, H.; Hafner, J. H.; Rinzler, A. G.; Colbert, D. T.; Smalley, R. E., Nanotubes as nanoprobes in scanning probe microscopy. Nature 1996, 384, (6605), 147-150.

5. Frederix, P. L. T. M.; Bosshart, P. D.; Engel, A., Atomic force microscopy of biological membranes. Biophysical Journal 2009, 96, (2), 329-338.

6. Fujisawa, S.; Ohta, M.; Konishi, T.; Sugawara, Y.; Morita, S., Difference between the forces measured by an optical-lever deflection and by optical interferometer in an atomic-force microscope. Review of Scientific Instruments 1994, 65, (3), 644-647.

7. Hsiao, C. C.; Peng, C. Y.; Liu, T. S., An optical lever approach to photodetector measurements of the pickup-head flying height in an optical disk drive. Measurement Science and Technology 2006, $17,2335-2342$.

8. Hutter, J. L.; Bechhoefer, J., Calibration of atomic-force microscope tips. Review of Scientific Instruments 1993, 64, (7), 1868-1873.

9. Butt, H. J.; Jaschke, M., Calculation of thermal noise in atomic-force microscopy. Nanotechnology 1995, 6, (1), 1-7.

10. Butt, H. J.; Cappella, B.; Kappl, M., Force measurements with the atomic force microscope: Technique, interpretation and applications. Surface Science Reports 2005, 59, (1-6), 1-152.

11. Butt, H.-J.; Kappl, M., Surface and interfacial forces. WILEY-VCH Verlag GmbH \& Co.: Weinheim, 2010.

12. Maskos, M., Influence of the solvent and the end groups on the morphology of cross-linked amphiphilic poly(1,2-butadiene)-b-poly(ethylene oxide) nanoparticles. Polymer 2006, 47, (4), 1172-1178.

13. Mueller, W.; Koynov, K.; Fischer, K.; Hartmann, S.; Pierrat, S.; Basche, T.; Maskos, M., Hydrophobic shell loading of PB-b-PEO vesicles. Macromolecules 2009, 42, (1), 357-361.

14. http://fluxxion.com/

15. Bagatolli, L. A.; Parasassi, T.; Gratton, E., Giant phospholipid vesicles: Comparison among the whole lipid sample characteristics using different preparation methods: A two photon fluorescence microscopy study. Chemistry and Physics of Lipids 2000, 105, (2), 135-147.

16. Dimitrov, D. S.; Angelova, M. I., Lipid swelling and liposome formation on solid surfaces in external electric fields. Progress in Colloid \& Polymer Science 1987, 73, 48-56.

17. Liebermann, T.; Knoll, W., Surface-plasmon field-enhanced fluorescence spectroscopy. Colloids and Surfaces a-Physicochemical and Engineering Aspects 2000, 171, (1-3), 115-130. 


\section{RESUlTS AND DISCUSSION}

\subsection{POLYMER MEMBRANES}

\subsubsection{IMPORTANCE OF POLYMERSOMES AND POLYMER MEMBRANES}

Synthetic amphiphiles such as poly(butadiene)- $b$-poly(ethylene oxide) (PB- $b$-PEO) block copolymers exhibit lipid-like properties and are known to be versatile building blocks of self-assembled polymer vesicles (polymersomes) (Figure 3.1, a-d). Various polymersomes have been used in encapsulation and delivery of various agents, as chemical reaction vessels and, more related to the research presented here, as model system to study the fundamental properties of membranes. ${ }^{1-5}$ The study of mechanical properties of polymersome membranes have been investigated mainly by micropipette aspiration experiments ${ }^{2,6-8}$ In contrast to previous studies of whole polymersomes, the interest of the present study is the investigation mechanical properties free-standing polymer membranes. The study was performed on pore-spanning $\mathrm{PB}_{130}-b-\mathrm{PEO}_{66}$ polymer membranes by means of AFM imaging and velocity dependent force indentation curves. Porous, hollow, bottomless, silicon nitride substrates were used to prepare pore-spanning polymer membranes that display a considerable robustness paired with local addressability (Figure 3.1e). Pronounced viscoelastic behavior, a strong resistance against area dilatation and tremendous stiffening upon cross-linking of the free-standing bilayer were observed. 
a)

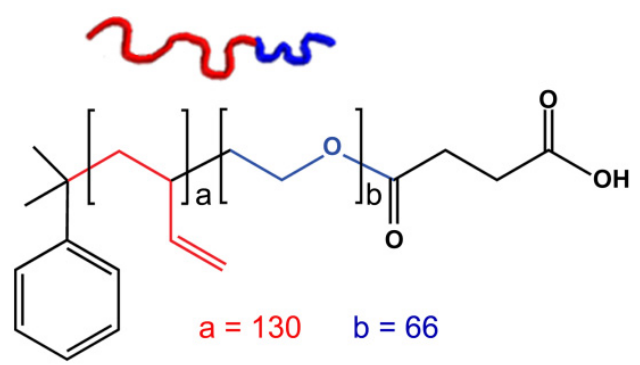

c)

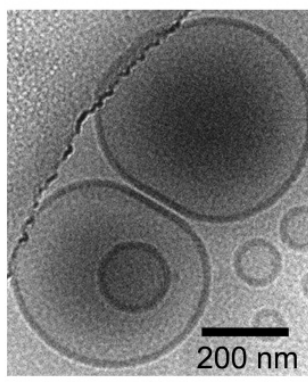

d) b)
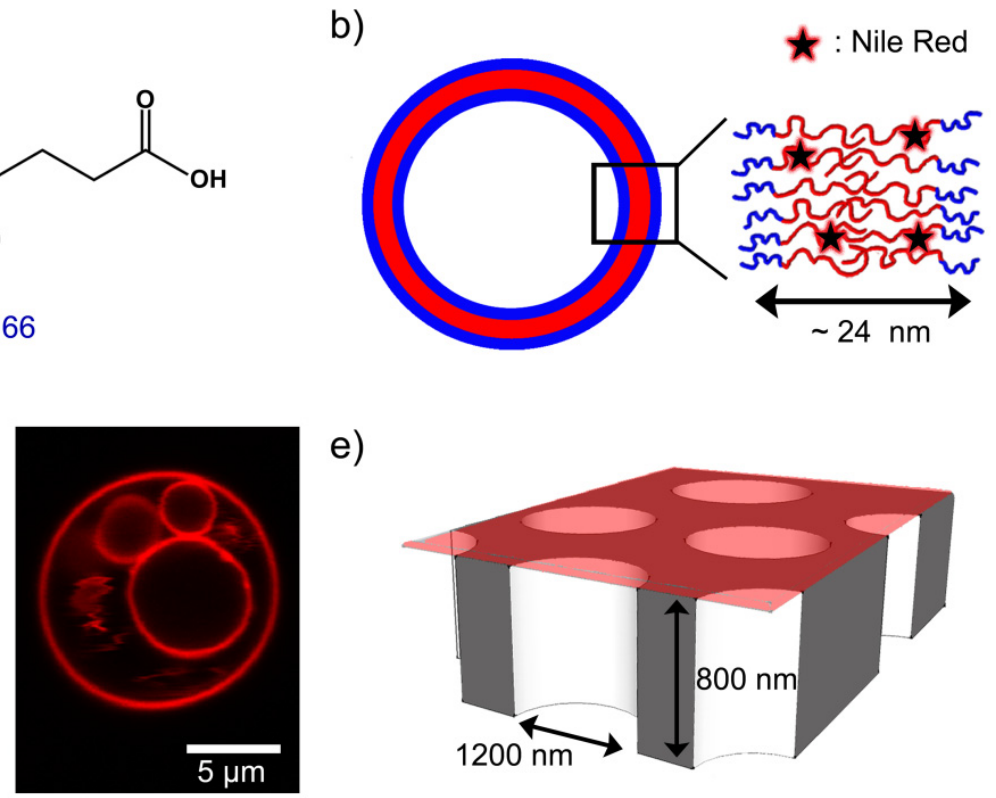

e)

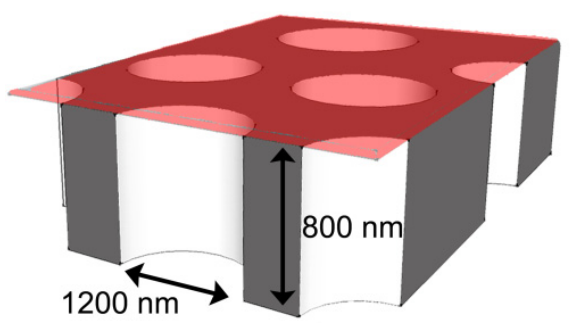

Figure 3.1 (a) Poly(butadiene)- $b$-poly(ethylene oxide) (PB-b-PEO) block copolymer chemical structure and its schematic representation. The subscripts $a$ and $b$ indicate the respective number of copolymer blocks: $a=130$ and $b=66$. (b) Schematic representation of a polymersome loaded with a lipophilic Nile Red Dye and the arrangement of the block copolymer forming the polymeric membrane, (c) cyroTEM of PB- $b$-PEO polymersomes, (d) CLSM image of a polymersome containing smaller polymersome inside of it and (e) schematic representation of a pore-spanning polymer membrane.

\subsubsection{PREPARATION OF PORE-SPANNING POLYMER MEMBRANES}

The polymer pore-spanning membranes were obtained from rupture of copolymer poly(butadiene)- $b$-poly(ethylene oxide) $\left(\mathrm{PB}_{130}-b-\mathrm{PEO}_{66}\right)$ polymersomes loaded with Nile Red dye $(0.25 \mathrm{wt} \%)$. The self-assembled polymer vesicles were prepared by gentle hydration of $\mathrm{PB}_{130}-b-\mathrm{PEO}_{66}$ films and characterized by cryogenic transmission electron microscopy (cryoTEM), light scattering as well as confocal laser scanning microscopy (CLSM) prior to their use in pore-spanning membrane preparation. A hydrophobic membrane thickness of 15-16 nm was estimated from CryoTEM micrographs. ${ }^{9}$ Porespanning polymer membranes were formed by vesicle adhesion to the surface, swelling and subsequent rupture on the porous support. The rupture process is facilitated by osmosis which takes place between the polymersomes, which contain $1 \mathrm{M} \mathrm{NaCl}$ solution, and deionized water environment of the measuring chamber. After addition of the polymersomes into the measuring chamber and their sedimentation onto the porous substrate, deionized water was added and the chamber left undisturbed for approximately 
1 hour. After pore-spanning membrane patches formed, the surface was gently rinsed with water to remove excess polymer material before further characterization.

\subsubsection{CHARACTERIZATION OF POLYMER MEMBRANES}

\subsubsection{FLUORESCENCE MICROSCOPY OF POLYMER MEMBRANES}

Once the pore-spanning polymer membranes were formed, the substrate was kept in aqueous environment. Fluorescence microscopy was used to determine if the polymer membranes have formed on the porous surface. It is the presence of Nile Red dye in the poly(butadiene) core of the membranes which gives the membrane patches their bright fluorescence intensity (Figure 3.2). When the polymersomes did not spread on the surface, no patches were observed after rinsing and the porous surface remained black under fluorescent light illumination. When polymer membrane patches did form, their exact location was assigned based on Cartesian coordinate system of the porous substrates (explained in the experimental section 2.2.2).
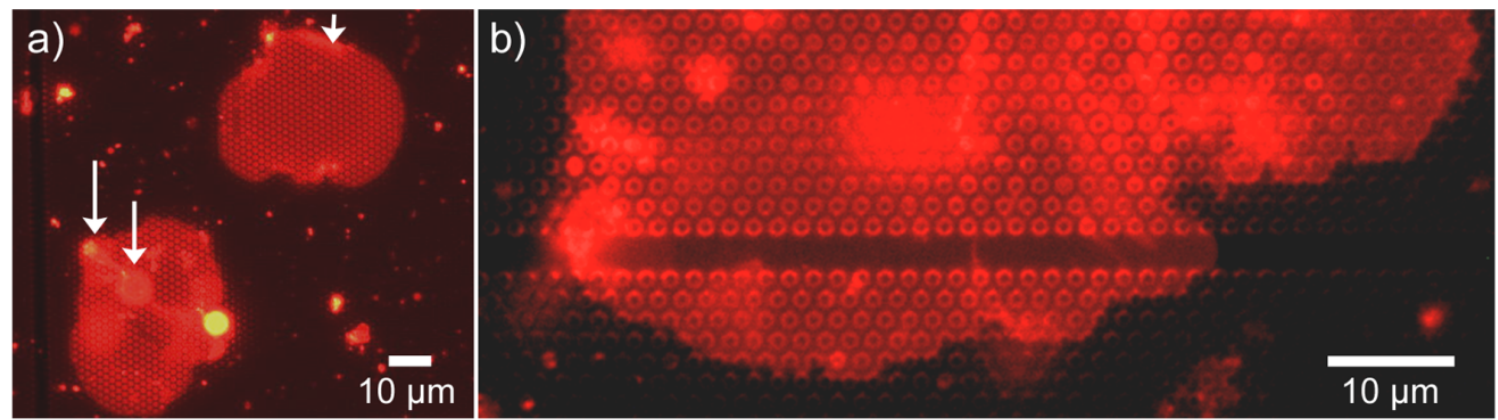

Figure 3.2 (a) Epifluorescence of pore-spanning polymer membranes prepared by PB- $b$-PEO polymersome swelling and rupture. In addition to locating membrane patches, fluorescence images show if the patches are uniform or if excess material is covering the patch (excess material is indicated by arrows). (b) Close-up of the porous substrate covered with a polymer membrane.

Besides locating the membrane patches, fluorescence images of the patches provide information about the amount of material deposited on the porous substrate. Single polymer membranes have uniform fluorescence intensity in contrast to membranes where excess polymer material deposits on the initial patches. The origin of the excess polymer material on the surface may be due to smaller vesicles, sometimes observed inside large polymersomes, or polymer material generated during polymersome rupture. Any excess polymer material is detected as increased fluorescence intensity (Figure 3.2a, arrows). 
Epifluorescence can be used to localize individual membrane patches, however, the membrane itself cannot be resolved. The resolution limit of the instrument and light scattering due to the pore geometry prevent direct membrane observation. It is not possible to determine if the membrane is spanning the pores or lining their interiors. Confocal laser scanning microscope (CLSM) was used in an attempt to determine if the membranes were pore-spanning. The advantage of CLSM imaging is the pinhole apertures which limit the amount of excitation light which interacts with the sample and prevent out of focus rays to reach the detector. Furthermore, single lines and threedimensional (3D) images of fluorescently labeled structures can be obtained. In order to verify if the polymer membrane was spanning the pores, a single line of fluorescence intensity was obtained from an image where some pores appeared to be covered with a membrane. The image with the fluorescence intensity overlay is shown in figure 3.3a. Although the fluorescence intensity difference between a non-covered pore (pore 12 in Figure 3.3a) and a pore which seems to be covered by a membrane (pore 3, Figure 3.3a) is clearly visible, the difference between a covered pore and a pore which seems to be lined by the membrane is not as apparent (pores 2 and 3, Figure 3.3a).

Another approach which was envisioned to confirm the pore-spanning character of the polymer membrane was to collect fluorescence intensity of a single line at various depths. The collected data was used to create a $z$ direction profile (z-stack) of fluorescence intensity (Figure 3.3b). Although the membrane cannot be seen due to the resolution limit of the instrument ( $800 \mathrm{~nm}$ in the $\mathrm{z}$ direction), the $\mathrm{z}$ direction fluorescence profile of the substrate provides some information about the sample. In comparison to non-covered pores (pores 1, 2, 7, 8, 9, 12, Figure 3.3b) the pores which appear covered in the $x-y$ image appear "filled" in the $\mathrm{z}$ direction profile (pores 3, 4, 5, 6, Figure 3.3b). Consequently, $\mathrm{z}$ direction profile of fluorescence intensity provides some visual indication about the pore-spanning nature of the membrane, however, additional characterization is needed to confirm that the pore is covered. 

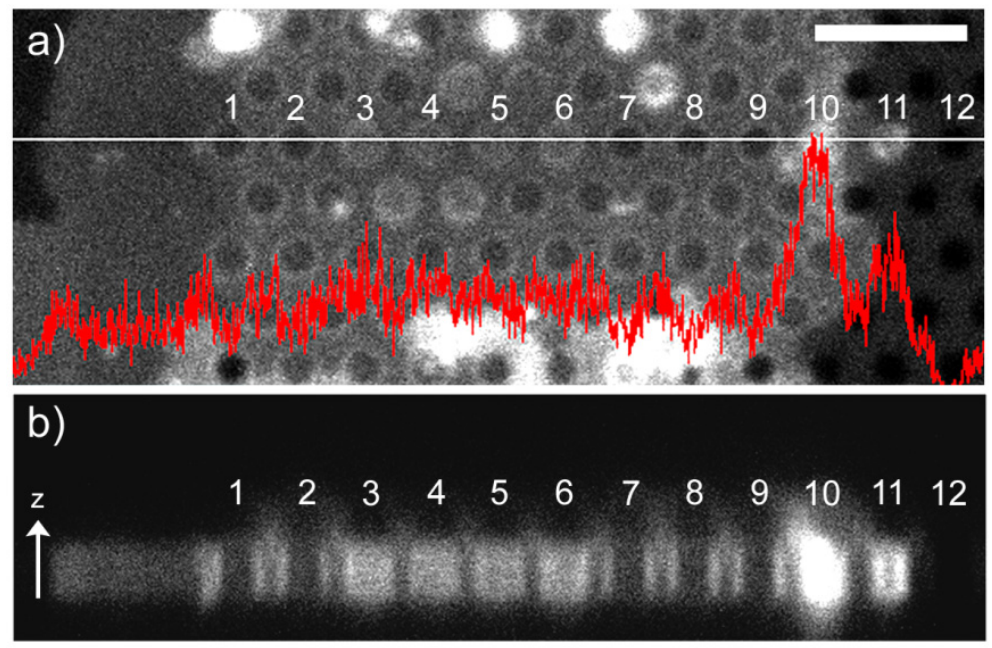

Figure 3.3 (a) CLSM image of a polymer membrane spanning a few pores with an overlay of fluorescence intensity profile obtained from the line drawn along the pores (b) z-direction profile of 12 pores where only pores 3,4,5 and 6 are covered with polymer membrane. Scale bar figure a: $5 \mu \mathrm{m}$, figure $b$ (height of arrow): $2 \mu \mathrm{m}$

CLSM z-stack results can also be presented in the form of a 3-dimentioanl (3D) image of the material on the surface. To construct a $3 \mathrm{D}$ image, consecutive focal plane images at varying $z$ positions are acquired and processed by the software to form a $3 \mathrm{D}$ image. These $3 \mathrm{D}$ images provide information which is not available from ordinary $2 \mathrm{D}$ images. For example, when looking at the $2 \mathrm{D} x-y$ image of figure $3.4 \mathrm{a}$, it is difficult to conclude that it is actually a polymersome with smaller polymersomes inside of it which adheres to the porous substrate and not a membrane patch with some excess material on top of it. In figure $3.4 \mathrm{~b}$, the $2 \mathrm{D} x-y$ image resembles a circular polymer patch where in fact it is a flattened out polymersome which has not yet ruptured. In both cases, the $3 \mathrm{D}$ images offer much more information about the true appearance of the structures on the surface. 
a)
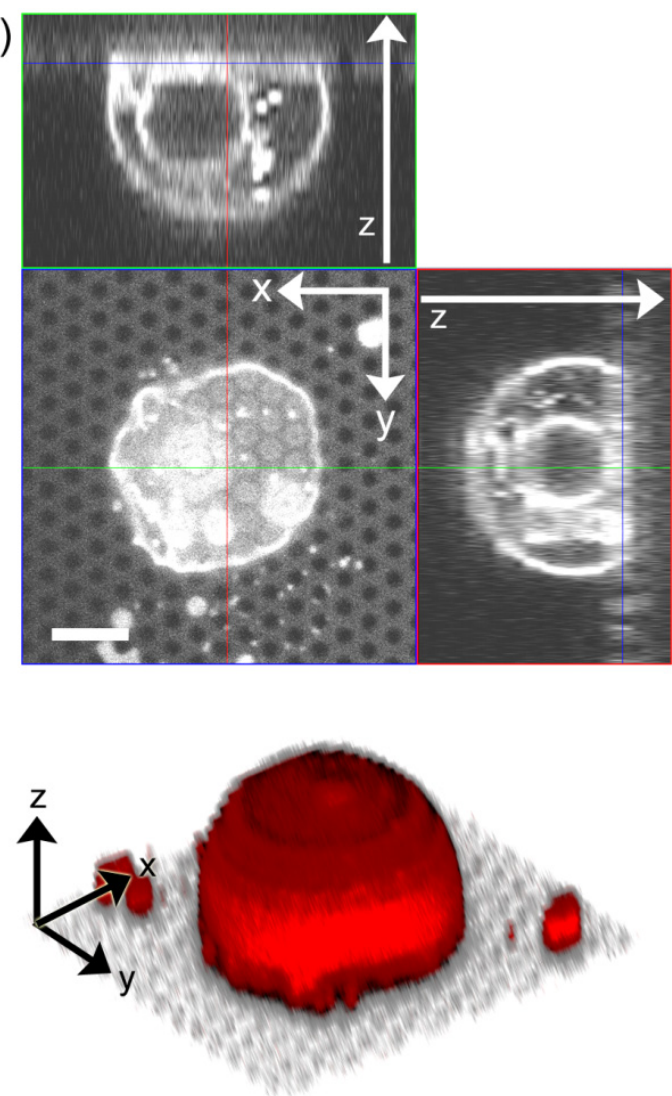

b)
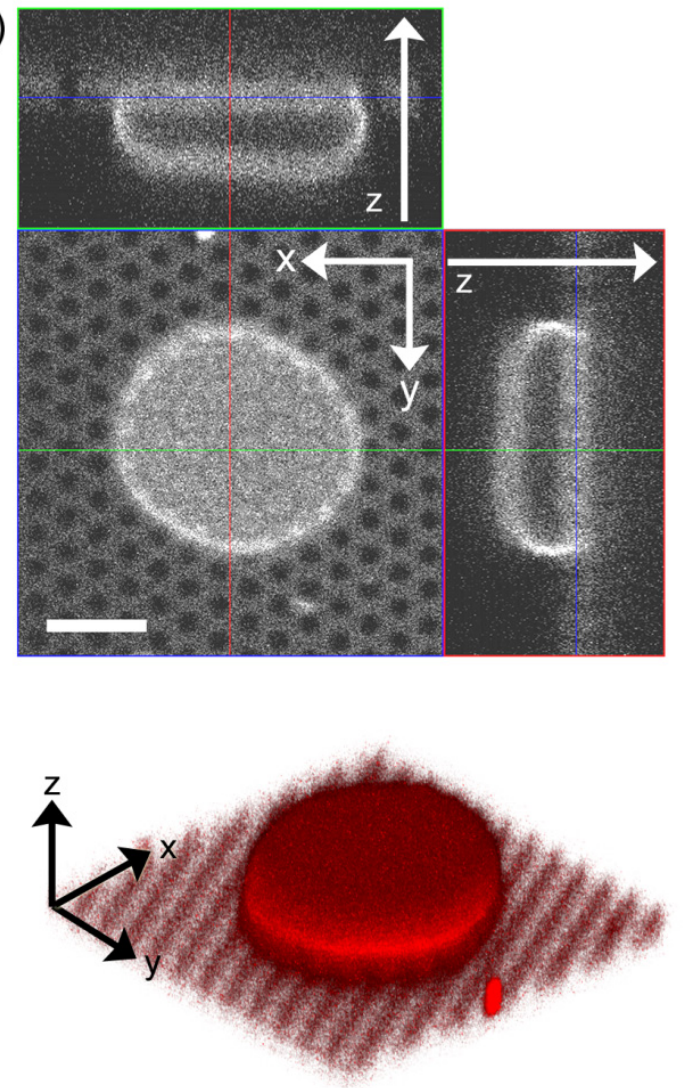

Figure 3.4 (a) An orthogonal section view of a polymer vesicle docked on a porous substrate with the 3D image below (scale bar: $5 \mathrm{um}$ ). (b) An orthogonal section view of a deflated vesicle on a porous surface with a 3D image below (scale bar: $5 \mu \mathrm{m}$ ).

In summary, fluorescence imaging provides direct information about membrane patch location on the substrate and the fluorescence intensity profiles can provide limited information about the membrane coverage of the pores. The $3 \mathrm{D}$ fluorescence images of the surface can be used to exclude areas where membrane patches have not formed yet (i.e. vesicles are still intact), however, additional characterization techniques must be used in combination with fluorescence microscopy to confirm the presence of a single polymer membrane on the substrate.

\subsubsection{AFM IMAGING OF POLYMER MEMBRANES}

Localization of the polymer membrane patches on the porous substrate by fluorescence microscopy was followed by atomic force microscopy (AFM) imaging. The porous silicon nitride substrates used in these experiments had ordered pore array and continuous flat silicon nitride areas (as shown in the experimental section). The coexistence of bare silicon nitride surface, membrane-covered flat silicon nitride 
surface and membrane-covered pores in a finite area allows for direct comparison of mechanical response to indentation between the membrane covered and non-covered surfaces. Contact mode images of the polymer membrane covering the flat areas of the substrate provided information about the thickness of the membrane. The height differences, ranging between 18 and $25 \mathrm{~nm}$, were in good agreement with the estimated membrane thicknesses obtained from CryoTEM images (provided by W. Mueller).
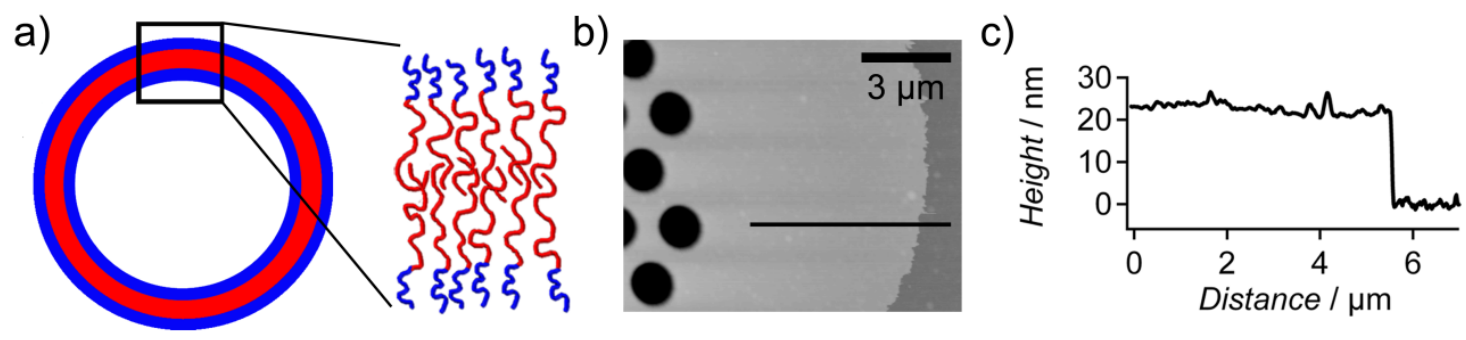

Figure 3.5 (a) Schematic representation of a polymersome with an enlargement showing the arrangement of PB-b-PEO copolymers forming the membrane. The thickness of the hydrophobic core is about $16 \mathrm{~nm}$ and the thickness of the hydrophilic edge is $4 \mathrm{~nm}$. (b) Contact mode AFM image of the polymer membrane spread on silicon nitride surface. (c) Height profile of the line in b) showing an overall membrane thickness of $c a .25 \mathrm{~nm}$.

In addition to verifying membrane thickness, AFM imaging was used to correlate fluorescence intensity of the material on the surface and its thickness. Fluorescent images where membrane patches exhibited two levels of fluorescence intensity were imaged by AFM. The height differences in the AFM images correlated well with the two fluorescence intensities such that $\sim 40 \mathrm{~nm}$ feature in AFM images matched the higher intensity fluorescence. An area where several polymersomes have ruptured was located by fluorescence microscopy and subsequently imaged by AFM. A line profile of the features visible in the AFM image (Figure 3.6) shows the differences in height that were measured. These correspond to multiples of approximately $20 \mathrm{~nm}$, which is approximately the thickness of a single polymer membrane. The brighter fluorescence areas corresponded to a patch measuring about $40 \mathrm{~nm}$ in thickness, while the less fluorescent areas corresponded to about $20 \mathrm{~nm}$ thickness. Based on the appearance of the patches and the expected thickness of a single polymer membrane (20-25 nm), the $40 \mathrm{~nm}$ thick feature is most probably due to a collapse of a polymersome on itself, resulting in the double membrane thickness. 
a)
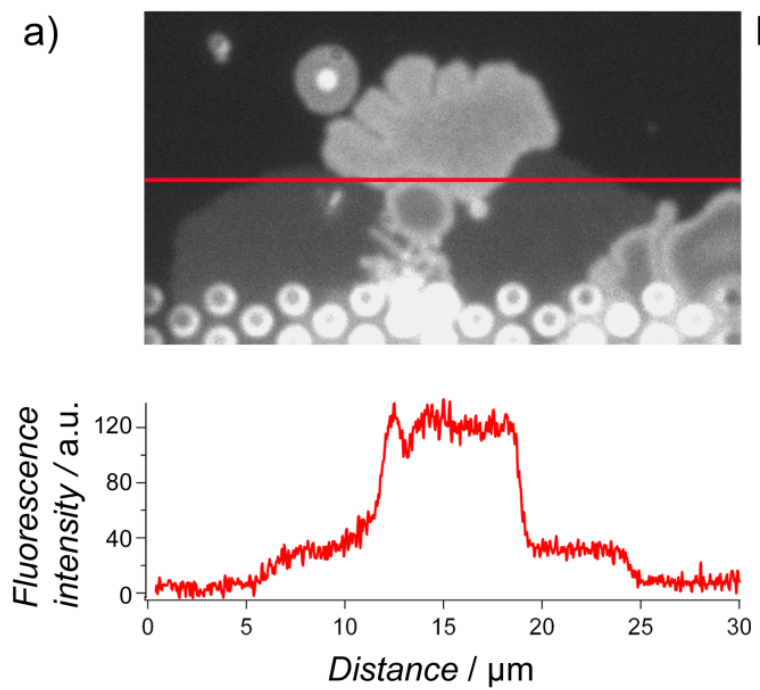

b)
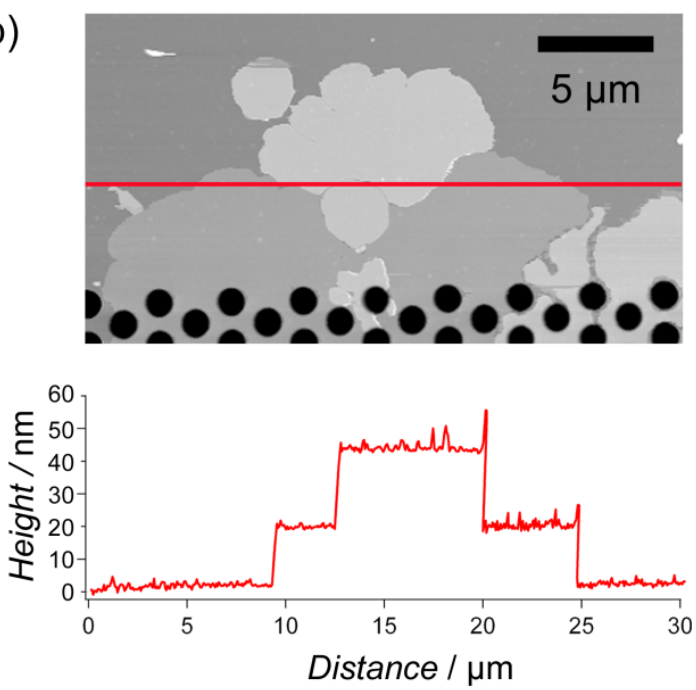

Figure 3.6 (a) Fluorescence image of polymer membrane patches at the boundary between flat and porous surfaces on a silicon nitride substrate. A line profile showing the fluorescence intensity is shown below the image (b) AFM contact mode image of the same area as in $a$. Below the image, a line profile obtained from the AFM image shows the height differences between the patches.

Similarly to the flat silicon nitride surface, the porous area of the substrate could also be imaged by AFM. However, the difference between membrane-covered pores and noncovered pores is not as apparent as the change in height observed at the boundary of membrane-covered and non covered flat surface. One of the reasons is large height difference between membrane thickness $\sim 25 \mathrm{~nm}$ and the depth of pores $\sim 800 \mu \mathrm{m}$. As the AFM tip is scanned over the porous substrate, it can penetrate much deeper into the pores that are not covered $(\mathrm{ca} .500 \mathrm{~nm})$, in comparison to the pores that are covered with a membrane (ca. $250 \mathrm{~nm})$. Therefore, the membrane-covered and bare pores can be distinguished when examining the height profile obtained from the AFM image (Figure $3.7 \mathrm{c})$.
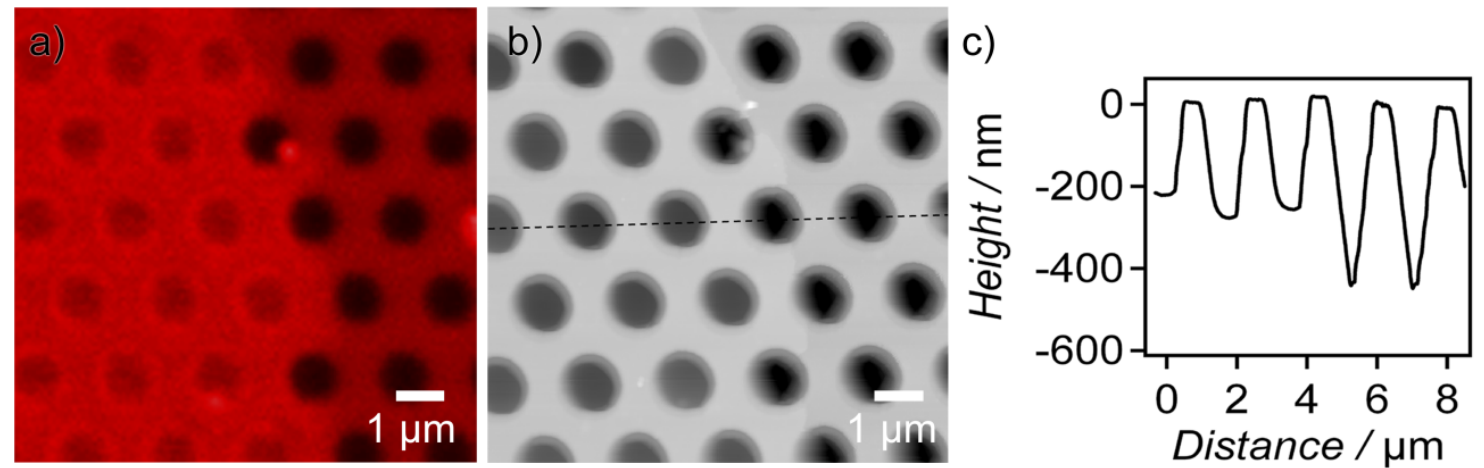

Figure 3.7 (a) CLSM images of polymer membrane spread on porous silicon surface. (b) Contact mode AFM image of the area shown in a where the porous silicon nitride substrate is partially covered with a polymer membrane. (c) The height profile of covered and uncovered pores. 
Care must be taken while imaging pore-spanning polymer membranes, so that the amount of force applied is kept low (below $1 \mathrm{nN}$ ) in order to avoid rupturing the membrane. Based on the results of indentation experiments discussed in the next section where the pore-spanning polymer membrane was indented well beyond $1 \mathrm{nN}$, it seems that it is not solely the imaging force which affects the integrity of the pore-spanning membrane but rather the combination of the applied force together with the raster scanning of the AFM tip over the surface. Membrane rupture during imaging has been observed and captured in an AFM image (Figure 3.8) which was obtained by scanning the surface starting from top of the image. The pore-spanning membrane was ruptured over several pores while the scanning was ongoing. Hence, the corresponding pores appeared to be covered only partially because the membrane rupture occurred only after the scan reached half of the pore; the upper part appeared covered because it was still was covered because the membrane was still intact, up until the line was scanned.

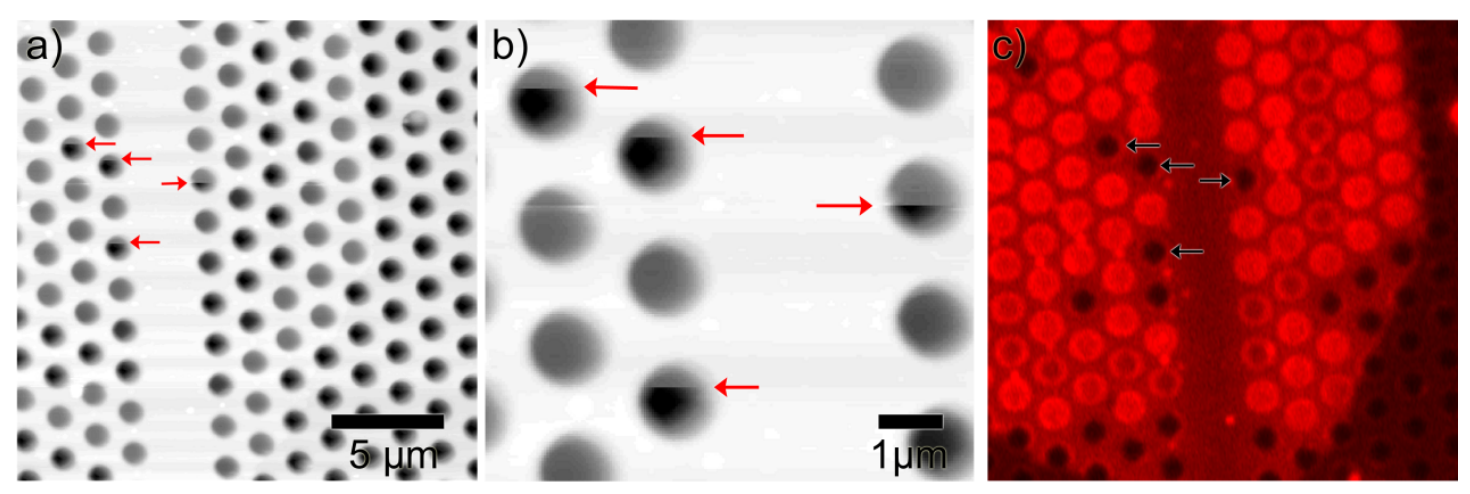

Figure 3.8 (a) Contact mode AFM image of a pore-spanning polymer membrane where individual pores were ruptured during imaging (shown with arrows) (b) enlargement of the porous area where the rupture of the membrane is visible, the arrow show the instant when the pore was uncovered. (c) CLSM image of the area imaged by AFM taken after imaging confirms that the membrane was ruptured during imaging.

Hole formation in a phospholipid bilayer membrane is an energetically unfavorable process because of the exposure of hydrophobic phospholipid tails to the aqueous environment. However, at the same time, the release of tension which accompanies hole formation is a driving force of this process. Membrane rupture starts with a hole formation and can be described by the model for formation of a circular hole in an incompressible sheet. ${ }^{10}$ The model states that the enthalpy of the system needs to be minimized: 
where $E$ is energy, $\tau$ is tension ( $\tau>0$ : tension, $\tau<0$ : compression), and $A$ is the area.

The energy associated with a circular hole in a sheet is defined by:

$E=2 \pi R \lambda$

where $R$ is the hole radius and $\lambda$ is the edge energy. The area difference between a membrane with a hole and an intact membrane is $\pi R^{2}$. According to the equation 3.1 , the enthalpy difference between an intact membrane and a membrane with a circular hole is defined by:

$$
\Delta H=2 \pi R \lambda-\tau \pi R^{2}
$$

The maximum value for $\Delta H$ occurs at $R^{*}$ where $R^{*}=\tau / \lambda$. Experimentally, when the hole formed in the membrane has a radius $\mathrm{R}<\mathrm{R}^{*}$, the hole will shrink and close, however, when $\mathrm{R}>\mathrm{R}^{*}$, the hole will expand and the membrane will rupture.

It is possible that during imaging of the pore-spanning membranes, the AFM tip pinned the membrane to the pore wall which initiated hole formation. Based on this model, it can be concluded that holes with radii $\mathrm{R}>\mathrm{R} *$ were formed and resulted in pore-spanning membrane rupture.

Ultimately, the combination of AFM imaging and fluorescence microscopy allowed for confirmation of the presence of the pore-spanning membranes.

\subsubsection{AFM FORCE SPECTROSCOPY OF POLYMER MEMBRANES}

Once the pore-spanning membranes were located by fluorescence microscopy and their pore-spanning character was confirmed by AFM imaging, force indentation experiments were performed at defined positions on the membrane patch. Force indentation experiments are performed by recording the deflection of the cantilever as it is brought into contact with the middle of the membrane-covered pore and then retracted to its initial position. The result is registered as cantilever deflection versus piezo displacement and is converted to force versus indentation curves once the spring constant of the cantilever is calculated. Prior to each set of force indentation curves, reference curves are acquired on a hard, non covered part of the silicon nitride surface. These reference curves are used for 
determination of 1) contact point of the AFM tip with the surface and 2) sensitivity of the photodiode. The output of the photodiode is a voltage, which has to be converted into a deflection signal (nanometers). When in contact with a hard surface, the deflection of the cantilever equals the piezo travel and the sensitivity is readily obtained by linear regression. Force curves recorded on the flat, a non-porous area of the substrate (Figure 3.9a) and on the center of a polymer membrane-covered pore (Figure 3.9b) are shown in figure 3.9.

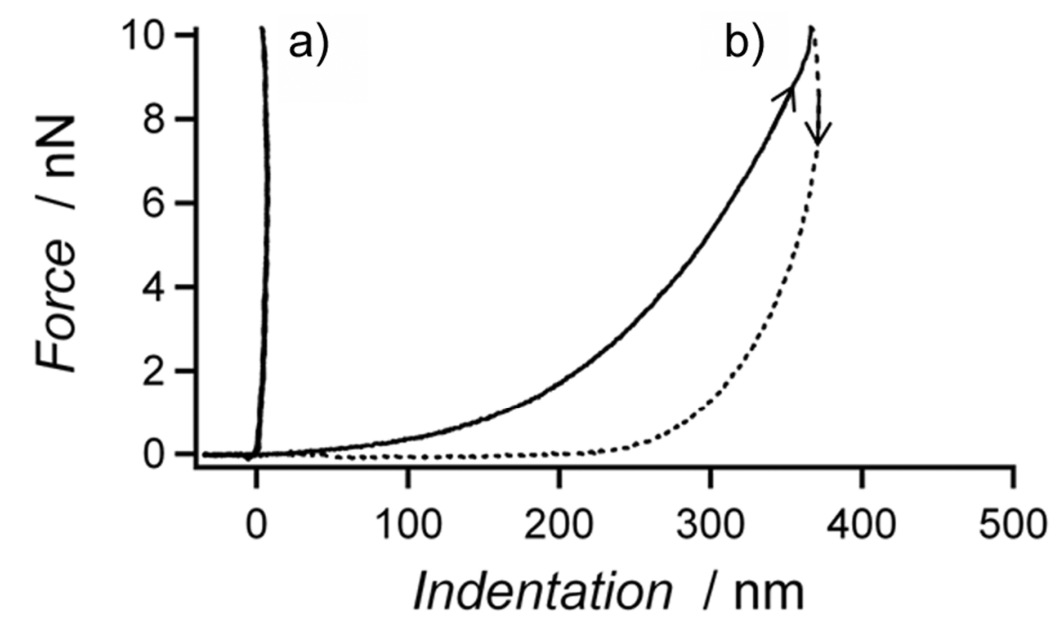

Figure 3.9 Representative force indentation curves. (a) A reference indentation curve performed on bare flat silicon nitride surface. (b) Typical force indentation curve of the polymer membrane suspended over a pore. Indentation (solid line) and retraction (dotted line) do not overlap as observed in the reference curve $a$.

The force curve performed on the flat silicon nitride surface (Figure 3.9a) shows essentially no indentation depth. In contrast, when the pore-spanning membrane is indented (Figure 3.9b), a nonlinear force indentation curve indicative of a very soft material is obtained. The deep indentation reaching several hundred nanometers without displaying rupture events confirms the high yield force of the polymer membranes to imposed stress accompanied by a rather soft response. Furthermore, a hysteresis between the indentation (solid line) and retraction (dotted line) is observed and is an indication of energy loss due to viscoelastic behavior of the material. Force indentation curves performed on the polymer-covered flat part of the silicon substrate exhibit similar characteristics to the curves performed on hard surfaces. As the AFM tip is moved 
towards the polymer covered flat surface, the polymer membrane is compressed until it ruptures (Figure 3.10) resulting in a noticeable kink in the indentation curve.

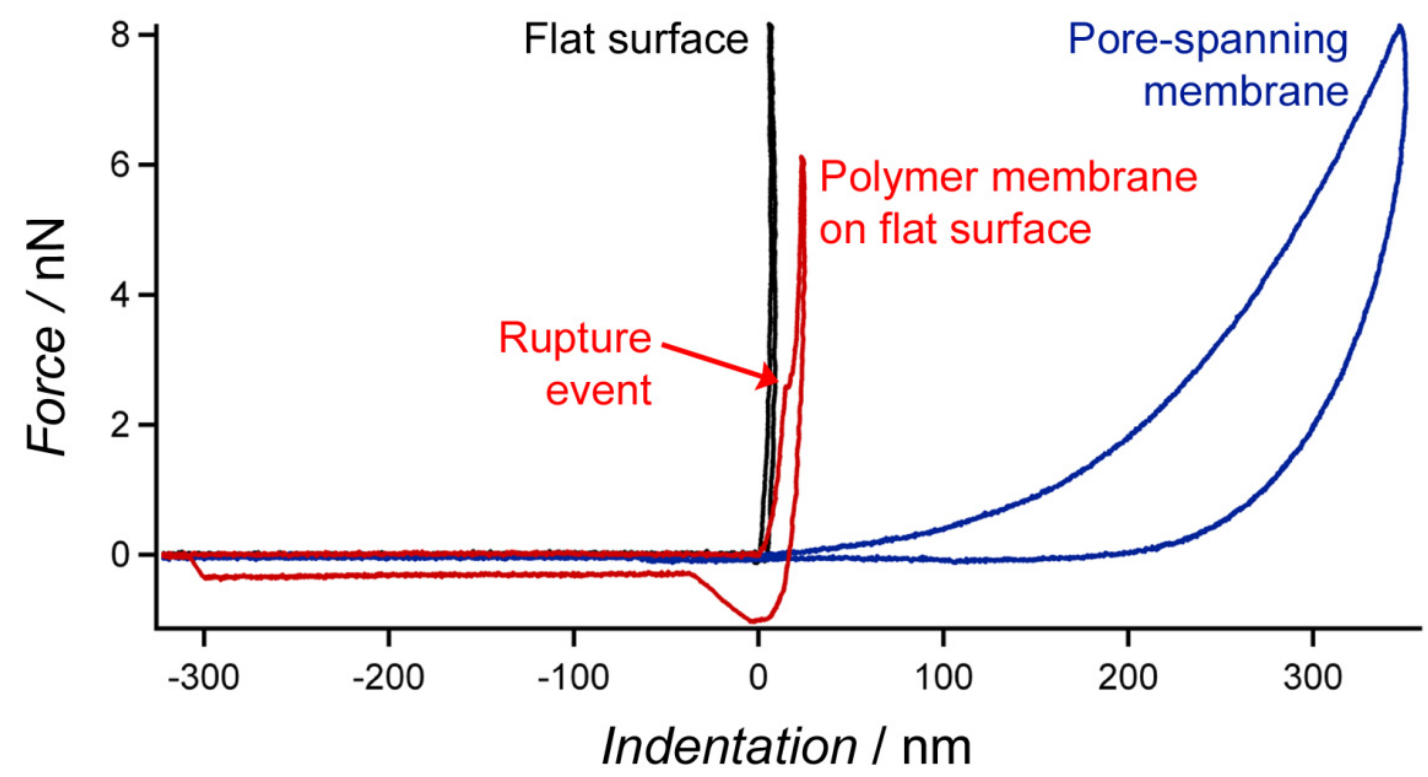

Figure 3.10 Difference between a force curve performed on the flat part of the substrate (black), force curve performed on the flat part covered with a polymer bilayer (red) and a force curve performed on bilayer-covered pore (blue). As the polymer membrane is compressed on the hard, flat surface the polymer ruptures (Rupture in the red curve).

Characterization results obtained from fluorescence imaging as well as from AFM imaging and from force spectroscopy experiments lead to the conclusion that the porespanning membrane preparation method has been successful. In-depth study of local membrane mechanics is preceded by a section on the theory of membrane mechanics.

\subsubsection{THEORY OF MEMBRANE INDENTATION}

The elastic response of a membrane to indentation comprises contributions from bending, stretching and lateral tension. While bending of the membrane has usually only a small influence on the force indentation curves as detailed below, stretching and lateral tension govern the elastic response of thin membranes to a large extent.

\subsubsection{BENDING}

The restoring force $F(h)$ of a clamped thin circular plate deflected by a flat cylindrical load with radius $R_{\text {load }}$ can be calculated from minimizing the bending 
energy $H=\pi \int_{0}^{R_{\text {pore }}} \mathrm{d} \rho \rho \kappa(\Delta h)^{2}:{ }^{11}$

$$
F=\frac{16 \pi \kappa}{R_{\text {pore }}^{2}}\left(1+\left(R_{\text {load }} R_{\text {pore }}^{-1}\right)^{2} \ln \left(R_{\text {load }} R_{\text {pore }}^{-1}\right)-3 / 4\left(R_{\text {load }} R_{\text {pore }}^{-1}\right)^{2}\right)
$$

where $\kappa$ denotes the bending modulus $\kappa=E d^{3} / 12\left(1-v^{2}\right)$, with $E$ the elastic modulus (Young's modulus), $v$ the Poisson ratio, $R_{\text {pore }}$ the pore radius, $d$ the membrane thickness, and $h$ the indentation depth. The two limiting cases, point load (Eq. 3.4a) and a homogeneous load applied to the center of the circular plate (Eq. 3.4b) can be readily regained: ${ }^{12}$

$\lim _{R_{\text {load }} \rightarrow 0} F=\frac{16 \pi \kappa}{R_{\text {pore }}^{2}} h$

$\lim _{R_{\text {load }} \rightarrow R_{\text {pore }}} F=\frac{64 \pi \kappa}{R_{\text {pore }}^{2}} h$

Assuming reasonable values for the parameters $\left(\kappa=10^{-19} \mathrm{~J}, \mathrm{R}_{\text {pore }}=600 \mathrm{~nm}\right){ }^{13}$ above, the apparent spring constant of the membrane is as low as $5.5 \cdot 10^{-5} \mathrm{Nm}^{-1}$. Considering that cantilever spring constants are of the order of $10^{-2}-10^{-3} \mathrm{Nm}^{-1}$, bending plays only a minor role in the mechanical response of polymer bilayers to indentation as it will become apparent from the experimental indentation curves.

\subsubsection{LATERAL TENSION AND PRE-STRESS}

Lateral tension arises due to the fact that membrane reservoir experiences friction on the non porous part of the silicon nitride substrate. By assuming a homogeneous lateral tension that also comprises a pre-stressed membrane and by neglecting bending of the membrane as argued above, an analytical expression as introduced by Bhatia and Nachbar ${ }^{14}$ as well as Deserno and coworkers ${ }^{15}$ can be found:

$$
h=\frac{F}{4 \pi \sigma}\left[1-\ln \left(\frac{F R_{\text {load }}}{2 \pi \sigma R_{\text {pore }}^{2}}\right)\right]
$$

where $\sigma$ is the lateral tension. 


\subsubsection{STRETCHING}

Stretching is described by membrane theory and is a major contribution to the elastic response at larger indentation forces. Assuming that the area dilatation $\delta A / A$ of membranes in two dimensions follows a 2-D Hookean law $\sigma=K_{\mathrm{a}}(\delta A / A)$ with tension $\sigma$ and the area compressibility $K_{\mathrm{a}} \cong E d,{ }^{16,17}$ obtained from $H=\int \sigma d A$ is the following cubic dependence of force on the penetration depth: ${ }^{18}$

$$
F(h)=\frac{\pi E d R_{\text {pore }}}{3}\left(\frac{h}{R_{\text {pore }}}\right)^{3}
$$

$d$ denotes membrane thickness. Begley et al. provide a force indentation relationship for a defined spherical indenter obtaining eq. (3.7) also with a cubic dependency: ${ }^{19}$

$$
F=\frac{9 \pi E d}{16 R_{\text {tip }}^{2}\left(R_{\text {pore }} / R_{\text {tip }}\right)^{\frac{9}{4}}} h^{3}
$$

Since most of the experimental force indentation curves show a cubic dependence on the indentation depth, it is assumed that stretching contributes significantly to the elastic response of the polymer bilayer.

\subsubsection{INDENTATION OF VISCOELASTIC MEMBRANES}

The flow behavior of polymer bilayer membranes is characterized by a surface viscosity for in-plane shear deformations, and an intermonolayer friction coefficient for the slip between the two leaflets of the bilayer. In the absence of covalent bonds between the polymers, the bilayer behaves as a two-dimensional liquid whose resistance against shear deformations is characterized by the surface shear viscosity $\eta$. Any relative motion between the two leaflets of a bilayer is opposed by a friction force. Besides the intermolecular friction, membrane flow into the pore upon indentation produces additional friction between the silicon nitride substrate and the bilayer. This gives rise to a lateral tension in the membrane. Hence, effective surface 
viscosity $\eta_{\mathrm{eff}}$ is defined to comprises all sources of lateral friction and energy dissipation. While pure lipid bilayers exhibit surface viscosities in the region of $10^{-4}$ $10^{-6} \mathrm{~Pa} \mathrm{~m} \mathrm{~s},{ }^{8,} 20$ entanglement and increase in chain length substantially increase surface viscosities by many orders of magnitude. As a rule of thumb, surface viscosity increases linearly with bilayer thickness if no entanglement occurs. ${ }^{13,21}$

A model representing the results shown in this work needs to account for two important experimental observations: the occurrence of a hysteresis between indentation and relaxation, which is indicative of energy loss due to friction upon indentation into the pore, and a decrease of energy dissipation with increasing indentation velocity. As a first approximation to account for the viscous loss upon indentation, a semi-empirical model comprising a Kelvin-Voigt model in series to a nonlinear spring $k_{\mathrm{m}}$ (Figure 3.11) representing the stretching of the bilayer $k_{\mathrm{m}}=9 \pi E d\left(16 R_{\mathrm{tip}}^{22 / 9} R_{\mathrm{pore}}^{9 / 4}\right)^{-1}$ was used. The Kelvin-Voigt model comprises the lateral tension and friction on the pore rim as a restoring force. Lateral tension as expressed by Eq. 3.5 can be safely approximated by a linear relationship between force and indentation depth at low forces giving rise to the apparent spring constant $k$.

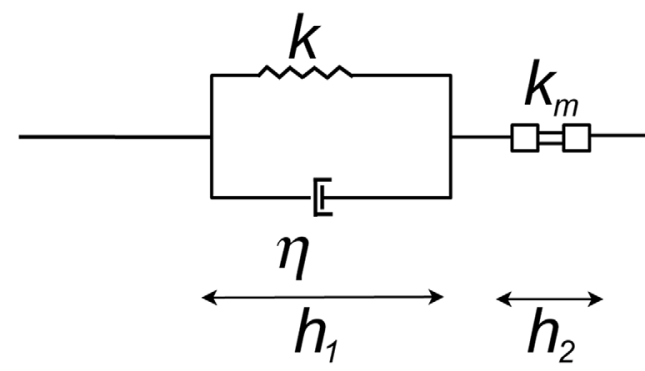

Figure 3.11 Modified Kelvin Voigt model in series with a nonlinear spring $k_{m}$ that represents in-plane stretching of the bilayer upon indentation. $h_{1}$ and $h_{2}$ denote the fractional indentations as a response to a stretching force.

The force exerted by the cantilever $F_{\mathrm{c}}=k_{\mathrm{c}}(v t-h)=k_{\mathrm{c}}\left(v t-h_{1}-h_{2}\right)$, with $k_{\mathrm{c}}$ the cantilever spring constant, $v$ the indentation velocity and $t$ the time, equals that produced by the membrane $F_{\mathrm{c}}=F_{\mathrm{m}}$ and the following is obtained:

$$
\begin{aligned}
& k_{\mathrm{c}}\left(v t-h_{1}-h_{2}\right)=k_{\mathrm{m}} h_{1}^{3} \\
& k_{\mathrm{c}}\left(v t-h_{1}-h_{2}\right)=k h_{2}+\eta \frac{\partial h_{2}}{\partial t}
\end{aligned}
$$


The set of equations is solved numerically by an Adams- Bashforth algorithm with the respective initial values for indentation $(h(0)=0)$ and relaxation $\left(h(0)=h_{\max }\right)$ resulting in the indentation force $F_{\mathrm{c}}=k_{\mathrm{c}}(v t-h(t))$ and relaxation force $F_{\mathrm{c}}^{\text {relax }}=k_{\mathrm{c}}\left(v\left(t_{\text {total }}-t\right)-h(t)\right)$. $t_{\text {total }}$ refers to the elapsed time during indentation reaching a maximal indentation depth of $h_{\max }$.

\subsubsection{FORCE SPECTROSCOPY STUDY OF PORE-SPANNING POLYMER MEMBRANES}

\subsubsection{UNTREATED PORE-SPANNING MEMBRANES}

Polymer membrane mechanics study was conducted by preparing pore-spanning membranes following the procedure described earlier in the text. Force indentation curves were performed at chosen areas of the polymer pore-spanning membrane patches and the experimental variables (i.e. indentation velocity, maximal indentation depth) were varied. Deep indentation depths, reaching several hundred nanometers, without displaying rupture events confirmed the high yield force of the polymer membranes to imposed stress. Hysteresis between indetation and relaxation curves was observed in all of the experiments. To investigate the nature of the hysteresis attributable to viscoelasticity of the polymer membrane, experiment where the membranes were indented at with various velocities were carried out.

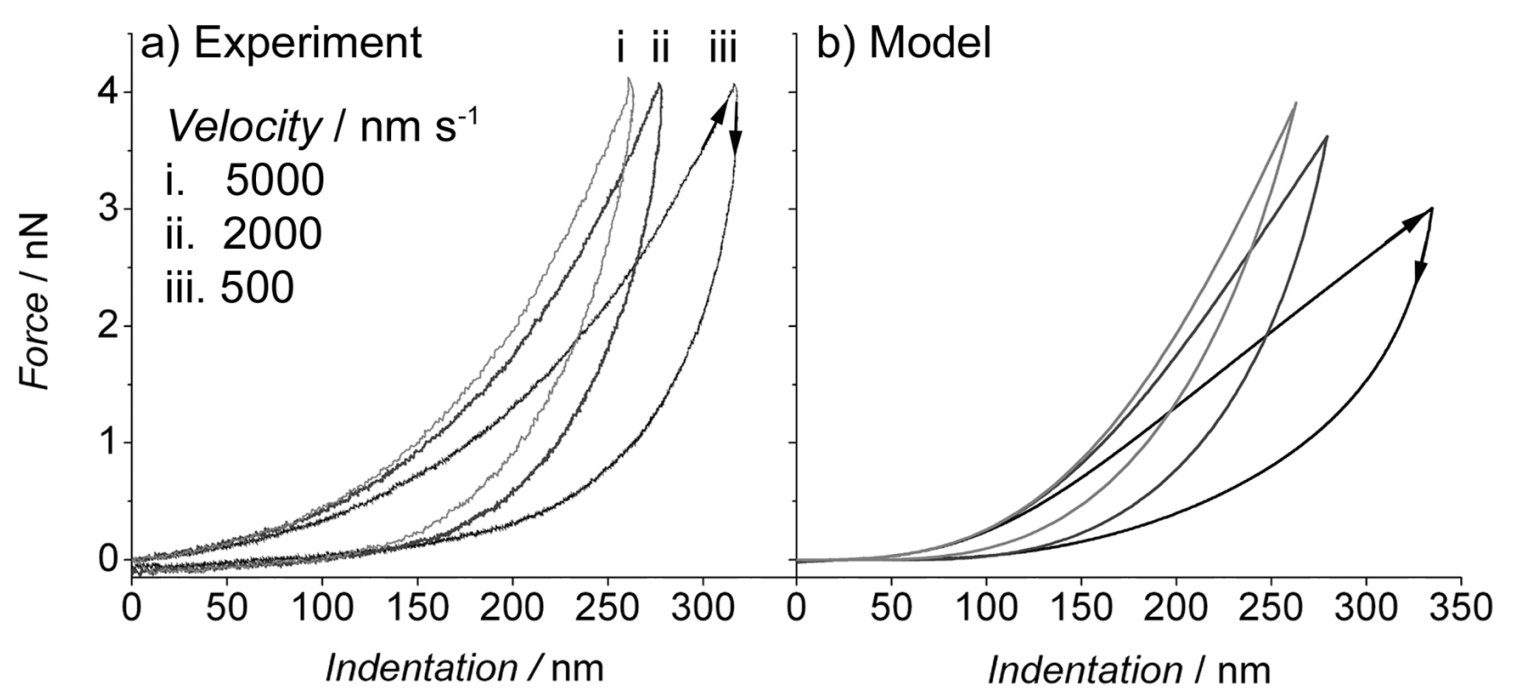

Figure 3.12 (a) Experimental force indentation curves performed with varying velocities (500-5000 $\left.\mathrm{nm} \mathrm{s}^{-1}\right)$. (b) Modeled force indentation curves. Experiments are performed on $600 \mathrm{~nm}$ radius silicon nitride porous substrates at constant maximal indentation force of $4 \mathrm{nN}$ with a silicon nitride cantilever $\left(k_{m}=2.63 \cdot 10^{-7} \mathrm{Nm}^{-}\right.$ ${ }^{1}, k=0.015 \mathrm{Nm}^{-1}, \eta=0.005 \mathrm{~Pa} \mathrm{~m} \mathrm{~s}, \tau=0.33 \mathrm{~s}$ where $\left.\tau=\eta / k\right)$. 
Assuming that the response of the polymer membrane upon indentation is governed mainly by stretching and pre-tension, as recently proposed for pore-spanning graphene layers and detailed in the theory section 3.1.4, Eq. 3.9 was used to fit the indentation of the curve (Figure 3.13).

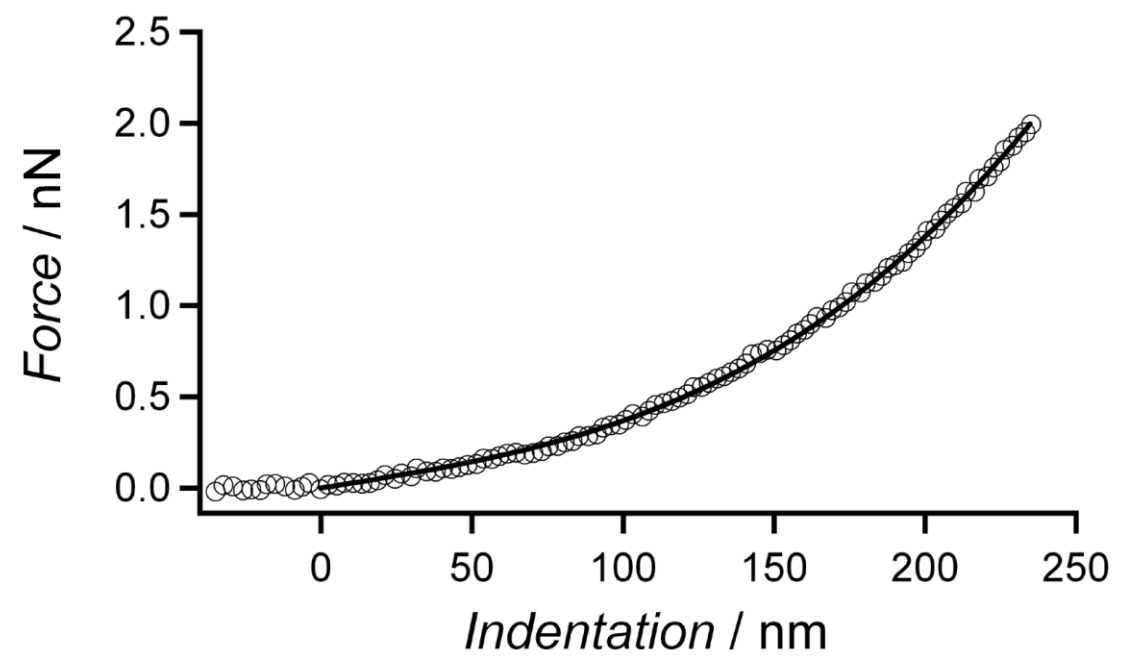

Figure 3.13 Force indentation curve ( $\circ$ markers) performed at $2000 \mathrm{~nm}^{-1}$ and $2 \mathrm{nN}$ maximal indentation force was fitted with a line obtained from equation 3.9.

Excellent agreement between experimental data (circles) and the model (Eq. 3.9) (solid line) was observed. Pre-tension $\sigma_{\text {pre }}$ was included to account for the free energy difference between the membrane adsorbed to the rim and the free-standing one. Note that the first term of Eq. 3.9 is a linear approximation of Eq. 3.5.

$$
F(h)=\sigma_{\text {pre }}\left(2 \pi R_{\text {pore }}\right)\left(\frac{h}{2 R_{\text {pore }}}\right)+\frac{9 \pi E d}{16 R_{\text {tip }}^{2}\left(R_{\text {pore }} / R_{\text {tip }}\right)^{\frac{9}{4}}} h^{3}
$$

The membrane prefers to be adherent on the rim, which essentially produces a lateral tension in the free-standing bilayer. After analysis of indentation curves performed at varying velocities, by fitting the indentation curve in the same manner as shown in figure 3.13, a mean pre-tension of $8.56 \cdot 10^{-4} \mathrm{Nm}^{-1}$ was found (Figure 3.14a) and an elastic modulus $E$ ranging from 1.68 to $8.65 \mathrm{MPa}$ depending on the indentation velocity (Figure $3.14 b)$. 
a)
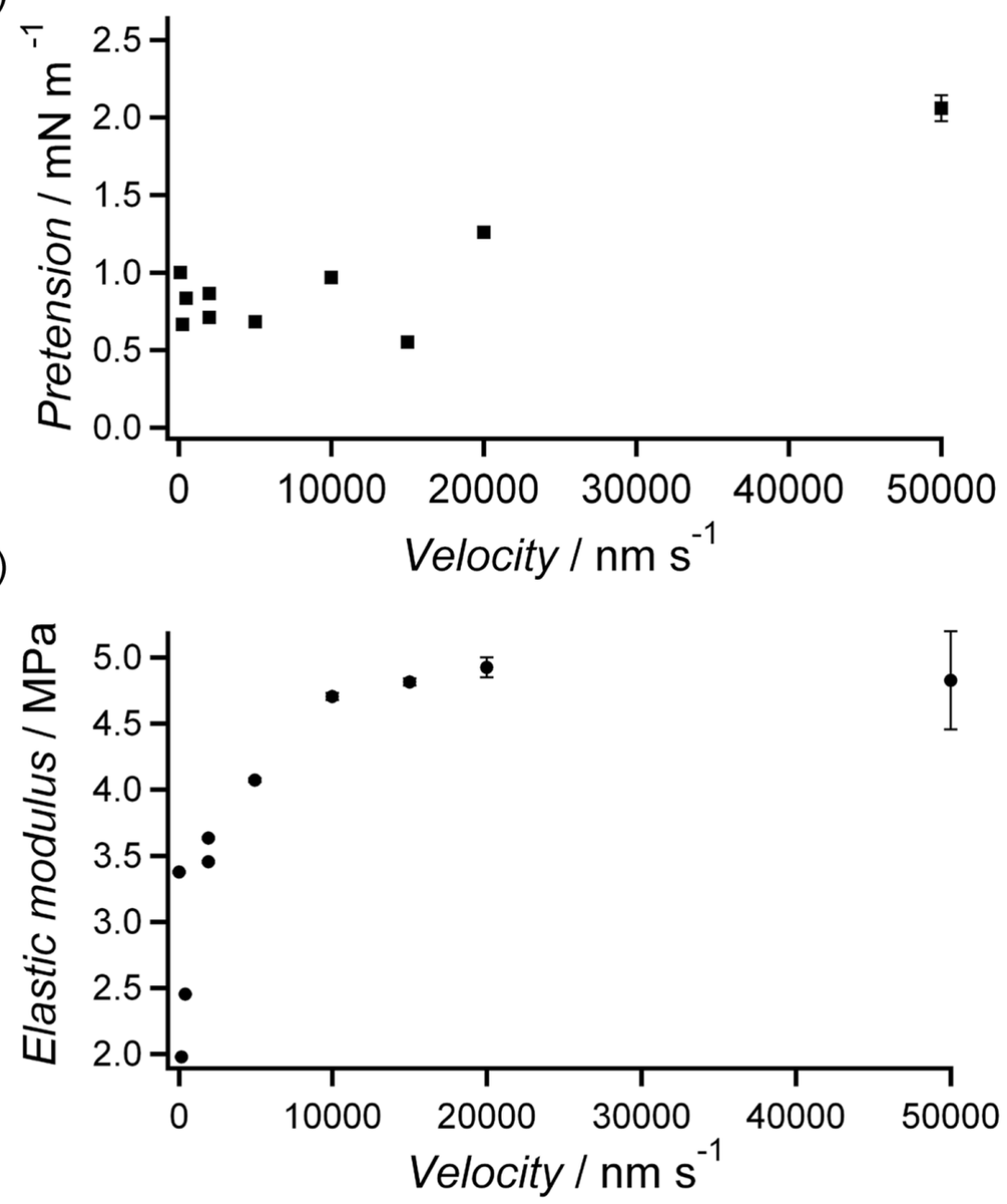

Figure 3.14 (a) Pre-tension of the polymer membrane determined at various indentation velocities and $2 \mathrm{nN}$ maximal indentation force. (b) Elastic moduli of a polymer membrane determined at various indentation velocities and $2 \mathrm{nN}$ maximal indentation force.

Figure $3.14 \mathrm{~b}$ shows the velocity dependence of $E$ leveling off at higher indentation velocities. This velocity dependence of $E$ is a first indication that the response of the polymer membrane is not entirely elastic but displays viscous contributions. In fact, a considerable hysteresis between indentation and retraction (relaxation) curves was observed, as shown in figure $3.9 \mathrm{~b}$. The hysteresis indicates that energy dissipation takes place and is dominated by frictional interactions between the polymer chains inside the membrane or attachment of the membrane to the substrate.

It was determined that the hysteresis between the indentation and retraction curves is decreasing with increasing indentation velocity (Figure 3.12a). In agreement with our finding that the area compressibility increases, decrease in energy dissipation with 
rising velocity was observed. The simplest way to model this behavior is to add a viscoelastic component to Eq. 3.9. The addition of a Kelvin-Voigt element in series to a nonlinear spring (Figure 3.11) that shows a cubic dependency of force on indentation depth, captures most of the experimentally displayed features. The force curves obtained from the theoretical model (Figure 3.12b) depict the hysteresis observed in the experiment and also correctly predict a decrease of the hysteresis with increasing indentation and retraction velocity. Relaxation time of $0.33 \mathrm{~s}$ and an area compressibility in the range of 0.030 to $0.156 \mathrm{Nm}^{-1}$ describes the viscoelastic properties of free-standing polymer bilayers best. The area compressibility is in good accordance to that of similar polymer membranes measured by micropipette suction experiments $\left(0.109 \mathrm{Nm}^{-1}\right)^{13}$ and $\left(0.088 \mathrm{Nm}^{-1}\right)^{8}$

Further investigation of the pore-spanning polymer membranes consisted in varying the maximal indentation force $F_{\max }$ (Figure 3.15).

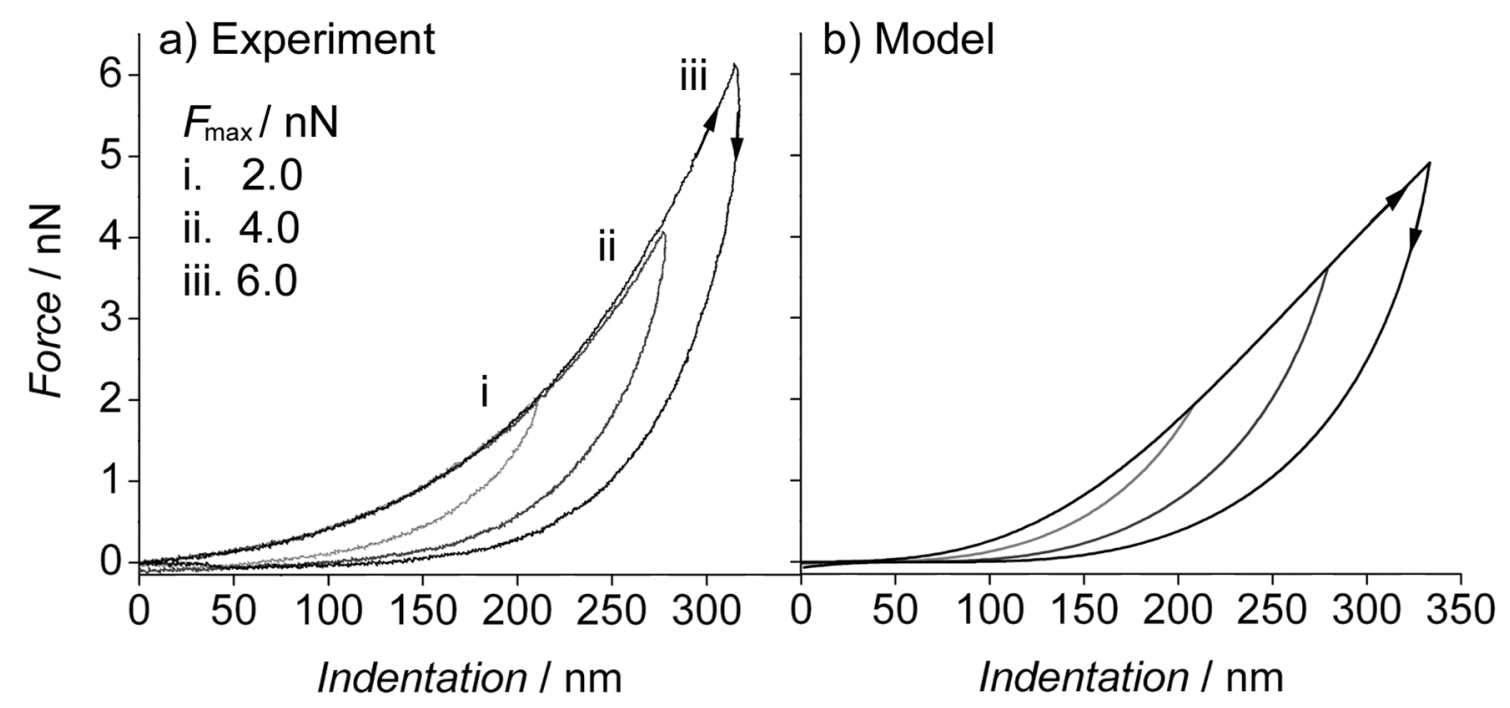

Figure 3.15 (a) Experimental force indentation curves performed with varying indentation forces (2.0-6.0 $\mathrm{nN}) . F_{\max }$ denotes the maximal force applied during an indentation-relaxation cycle. (b) Modeled force indentation curves. Experiments are performed on $600 \mathrm{~nm}$ radius silicon nitride porous substrates at a constant velocity of $2000 \mathrm{~nm} \mathrm{~s}^{-1}$ with a silicon nitride cantilever $\left(k_{m}=2.63 \cdot 10^{-7} \mathrm{Nm}^{-1}, k=0.015 \mathrm{Nm}^{-1}, \eta=\right.$ $0.005 \mathrm{~Pa} \mathrm{~m} \mathrm{~s}, \tau=0.33 \mathrm{~s}$ where $\tau=\eta / k$ ).

An increase of hysteresis with increasing indentation force was noted. Naturally, a larger indentation depth results in a more pronounced hysteresis as it is predictable from the varying velocity experiment. More precisely, under both conditions - low velocity and high indentation depth - the AFM tip stays in contact with the membrane for a longer period of time resulting in an extensive rearrangement of the polymer 
chains. The curves obtained from modified Kelvin-Voigt model also captured this behavior (Figure 3.15b). Although the modeled force indentation curves were not identical to the experimental results, the principal characteristics (i.e. increasing hysteresis with increasing contact time of the tip with the membrane) were maintained. Importantly, reproducible force indentation curves on the same pore confirm that the system regains its initial state regardless of velocity of indentation or the indentation depth. This finding is an important indication of the polymer membrane does not undergo fatigue.

\subsubsection{CROSS-LINKED PORE-SPANNING MEMBRANES}

An important feature of the polymer membrane is the possibility to chemically stiffen the bilayer by forming interchain cross-links. Polymerization offers a way to control the viscoelastic response of the material. ${ }^{7}$ It also presents the opportunity to investigate how the entanglement of the polymer chains in the hydrophobic core of the membrane affects the hysteresis observed in the force indentation curves. Crosslinking of the poly(butadiene) blocks of the polymer chains was achieved by exposing the polymer membrane spanned over the porous substrate to UV light after addition of a benzophenone photoinitiator. The results of this permanent modification were immediately visible in the AFM images where the indentation of the cross-linked membrane during imaging was considerably shallower than that of the native unmodified polymer membrane (Figure 3.16)
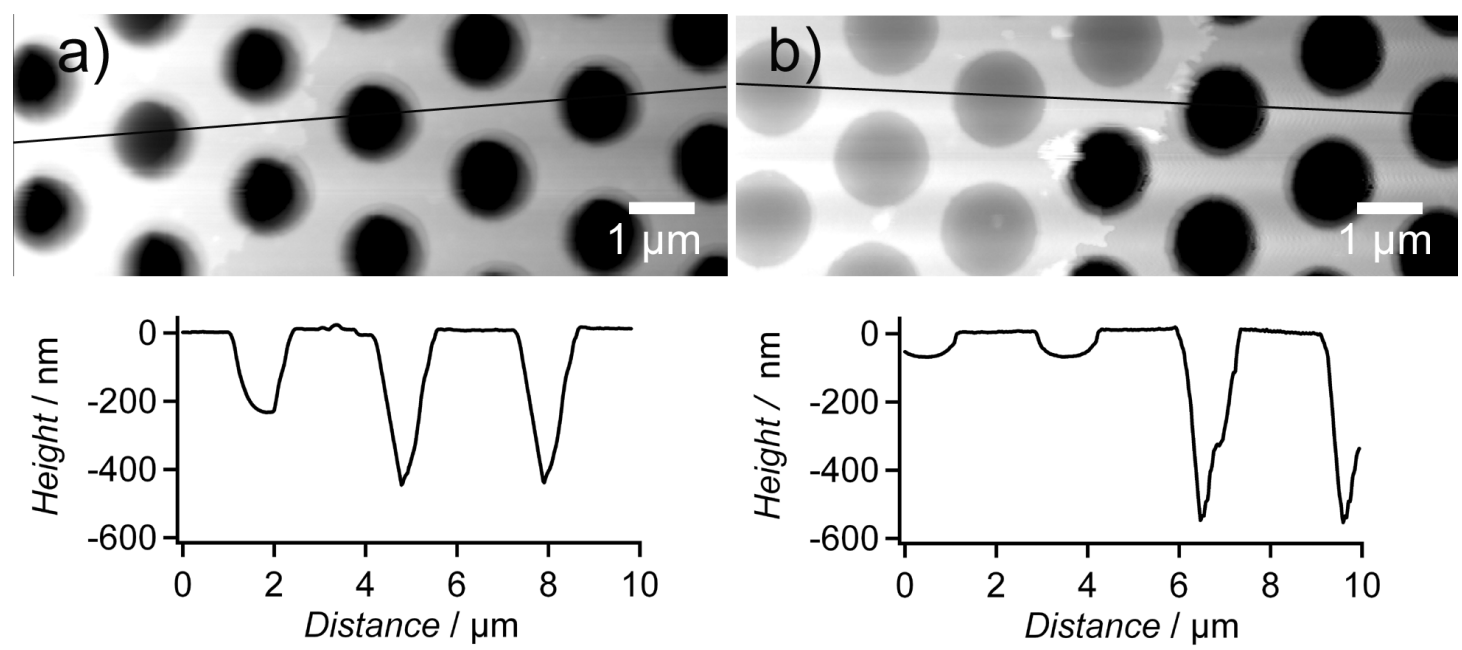

Figure 3.16 Contact mode AFM images and the corresponding height profiles of (a) untreated $\mathrm{PB}_{130}-b$ $\mathrm{PEO}_{66}$ pore-spanning membrane imaged at $0.8 \mathrm{nN}$ force and (b) cross-linked $\mathrm{PB}_{130}-b-\mathrm{PEO}_{66}$ imaged at 1.4 $\mathrm{nN}$ force. 
The AFM contact mode images show a dramatic change in the response of the polymer membrane to the AFM tip during the scan. Even through the membrane in figure $3.16 \mathrm{~b}$ is imaged at a force of $1.4 \mathrm{nN}$, which is almost the double of the imaging force of the non UV-treated membrane $(\sim 0.8 \mathrm{nN})$, the cross-linked membrane is indented less than $100 \mathrm{~nm}$ into the pores. In addition to the change in indentation depth during imaging, the membrane has decreased in thickness by $\sim 15 \mathrm{~nm}$, which is visible in the line profiles of figure $3.17 \mathrm{~d}$. The membrane shrinkage was also observed in the fluorescence images before and after the UV treatment (Figure 3.17e-f). Cracks in the polymer membrane resulting from polymer membrane contraction after UV treatment are marked with arrows.

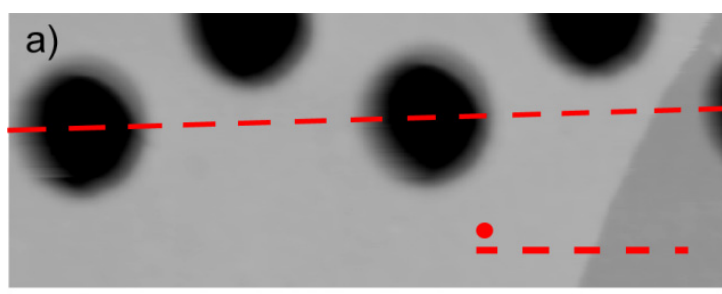

b)
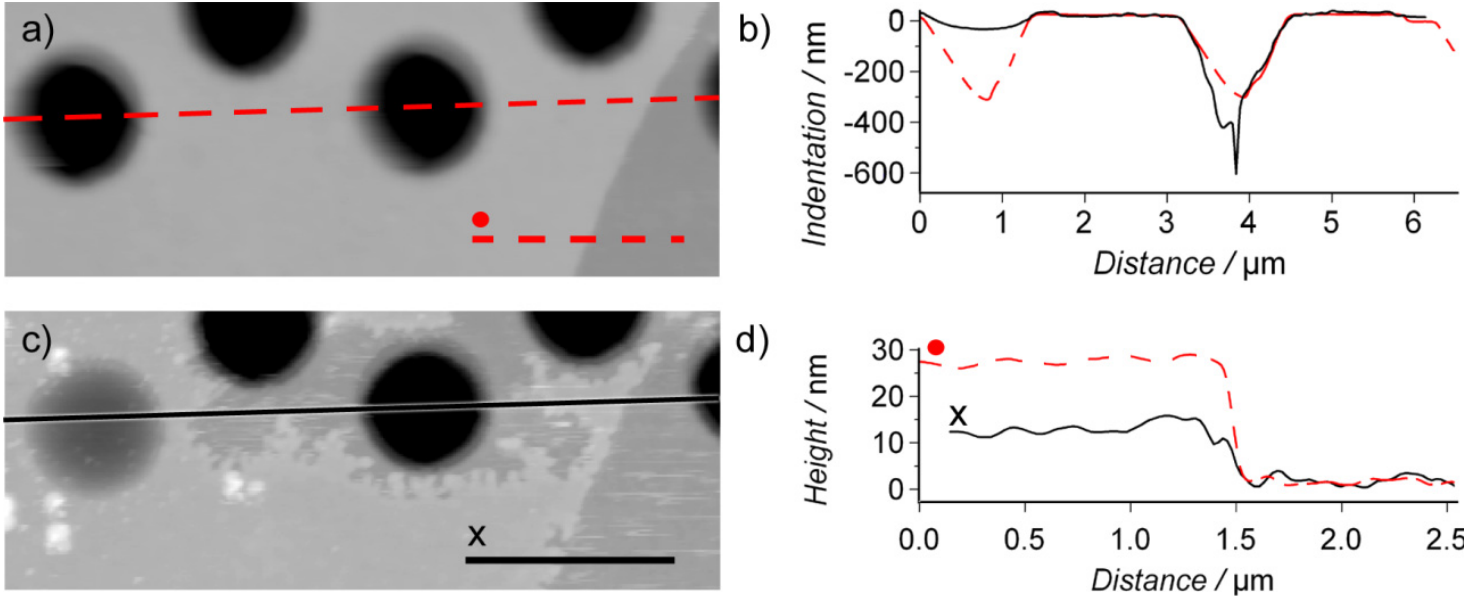

d)
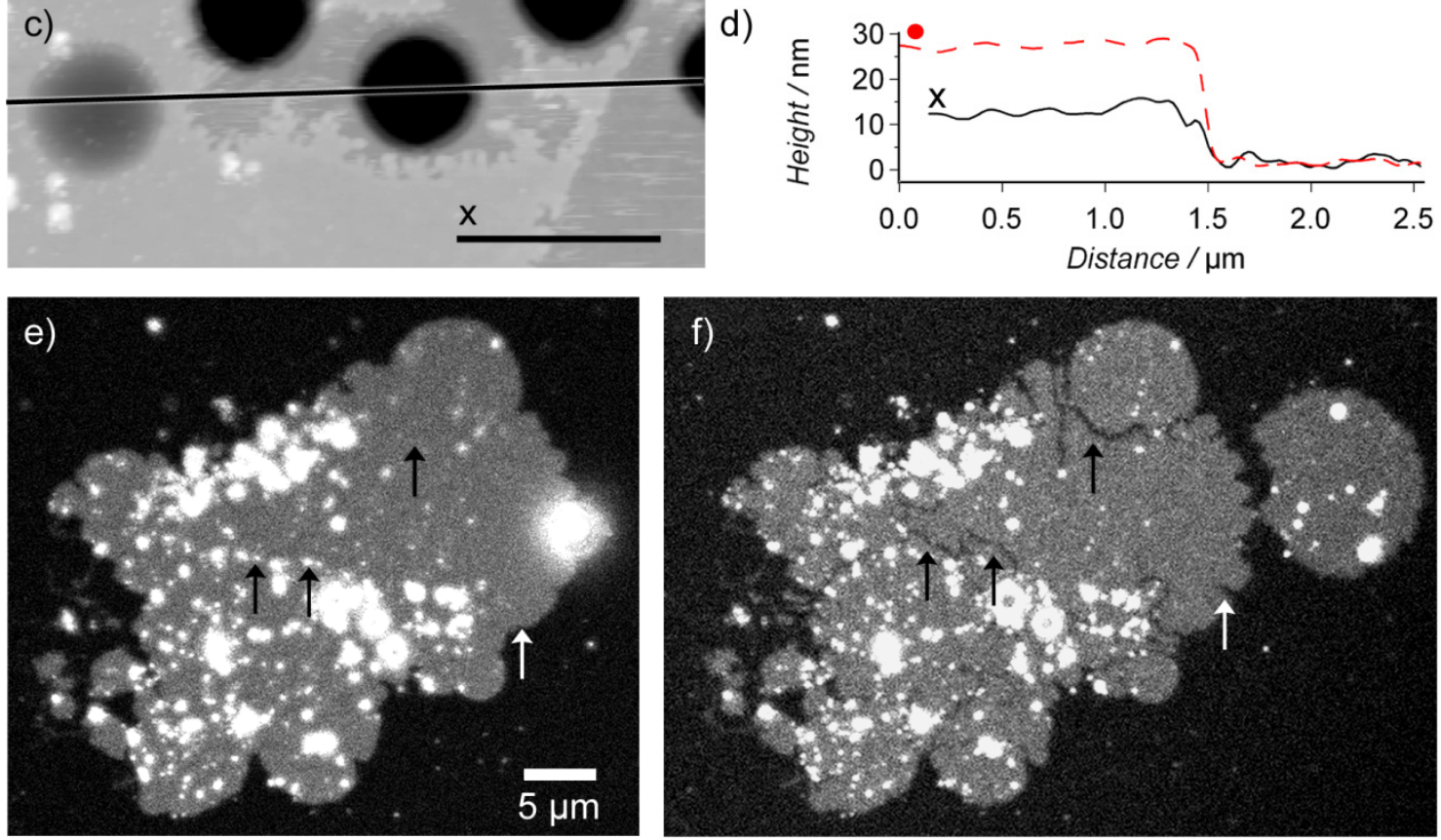

Figure 3.17 Contact mode AFM images of (a) untreated $\mathrm{PB}_{130}-b-\mathrm{PEO}_{66}$ pore-spanning membrane, (c) cross-linked $\mathrm{PB}_{130}-b-\mathrm{PEO}_{66}$ pore-spanning membrane and (b) their corresponding height profiles. Fluorescence images of (e) untreated membrane on flat surface and (f) UV cross-linked membrane on flat surface with membrane cracks marked with an arrow. 
After locating the UV cross-linked pore-spanning membranes patches on the porous surface, force indentation experiments were performed. A direct comparison of the response of a non-treated and cross-linked membranes to the indentation is shown in figure 3.18 .

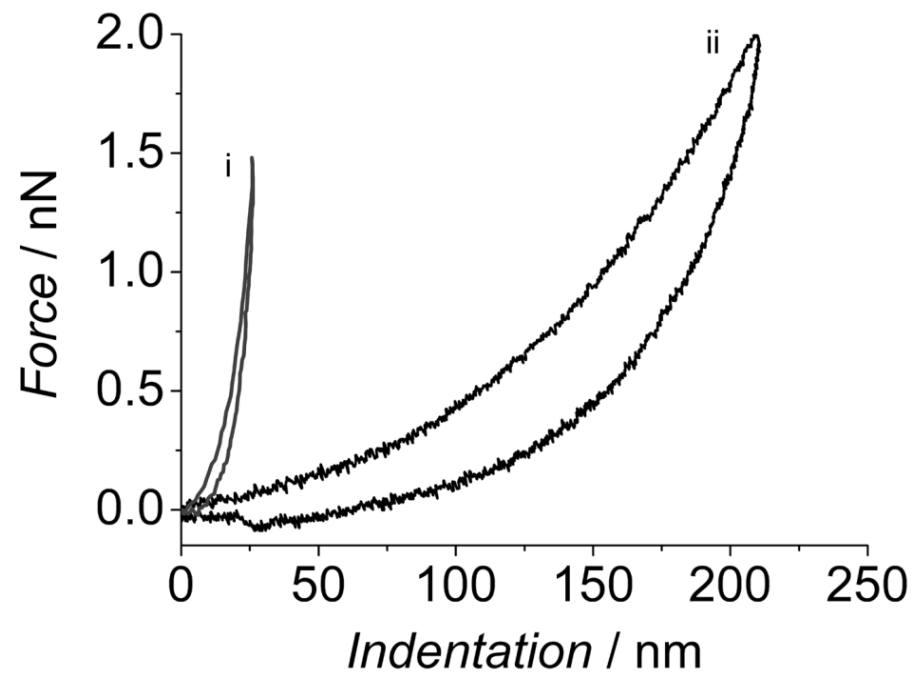

Figure 3.18 Overlay of two force indentation curves performed on (i) cross-linked and (ii) untreated $\mathrm{PB}_{130^{-}}$ $b-\mathrm{PEO}_{66}$ pore-spanning membranes. Force indentation curves were performed at $2000 \mathrm{~nm}^{-1}$ velocity.

Both, untreated and cross-linked membranes were indented with the same velocity and with similar maximal indentation force. The untreated membrane (Figure 3.18, curve ii) showed an elastic response and a pronounced hysteresis. In contrast, the cross-linked membrane (Figure 3.18, curve i) showed much stiffer mechanics and reduced hysteresis.

The analysis of the force indentation curves performed on the cross-linked membranes showed a tremendous increase of area compressibility values where the untreated membranes displayed $K_{\mathrm{a}}$ of $0.03 \mathrm{Nm}^{-1}$ and a $K_{\mathrm{a}}$ value of $64 \mathrm{Nm}^{-1}$ was determined after cross-linking. In fact, the shrinking of the material gives rise to smaller membrane extensibility particularly in the $\mathrm{x}-\mathrm{y}$ plane. Furthermore, energy dissipation of cross-linked membranes upon indentation is substantially smaller (tens of attojoules), than in noncovalently assembled bilayers (hundreds of attojoules) (see summary in Figure 3.22).

Similarly to the force indentation curves performed on untreated polymer membranes, fit from Eq 3.9 was used to fit the force indentation curves aquired on UV-treated 
membranes (Figure 3.19). As it was the case with the untreated polymer membranes, the fit is in excellent agreement with the experimental data.

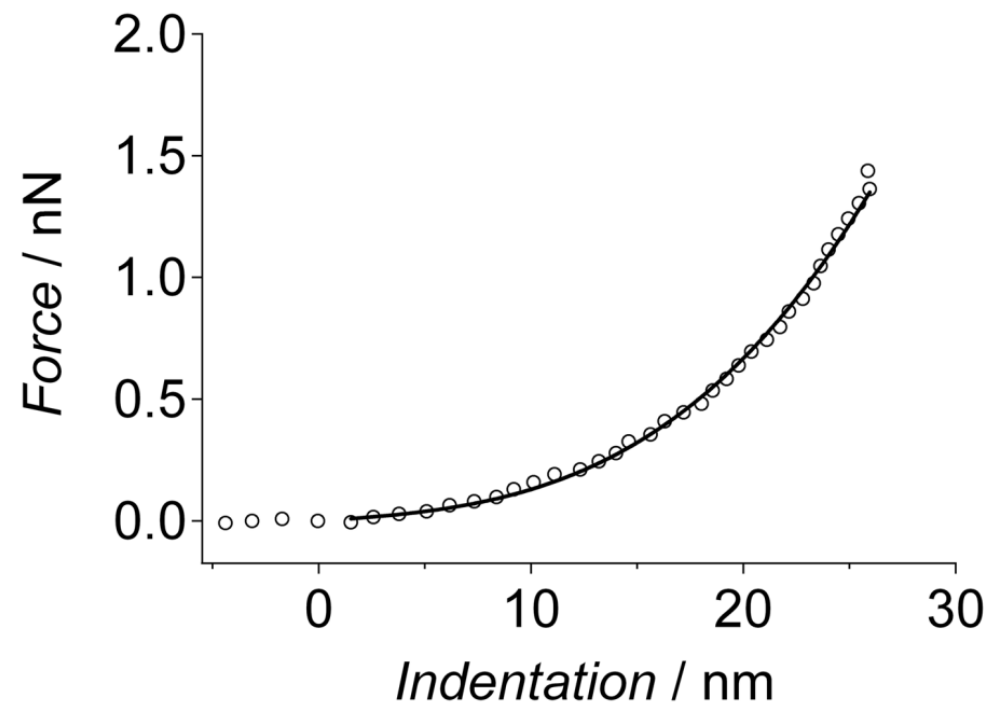

Figure 3.19 Force indentation curve of the cross-linked $\mathrm{PB}_{130}-b-\mathrm{PEO}_{66}$ membranes ( $\circ$ markers) with a fit (solid line) obtained with Eq. 3.9.

Young moduli values of the UV-treated membranes provided by the Eq. 3.9 fit are in the giga pascal range, which are almost three orders of magnitude higher than the $E$ values obtained from fits of non-treated polymer membranes (mega pascal values). $E$ value ranges for UV-treated and untreated polymer membranes are compiled in Table 3.1. UV-cross linking of the poly(butadiene) membrane core confirms that the polymer chain entanglement affects the fundamental mechanical properties of the polymer membrane. Both the hysteresis and the Young modulus of the polymer have been found to change upon cross-linking.

Additional force indentation experiments with varying maximal indentation force $F_{\max }$ were performed (Figure 3.21) to investigate the hysteresis between indentation and relaxation curves. Even though the hysteresis of the cross-linked membranes was less pronounced than in untreated polymer membranes, it was still detectable and followed the trend where deeper indentation resulted in higher hysteresis (Figure $3.21)$. 


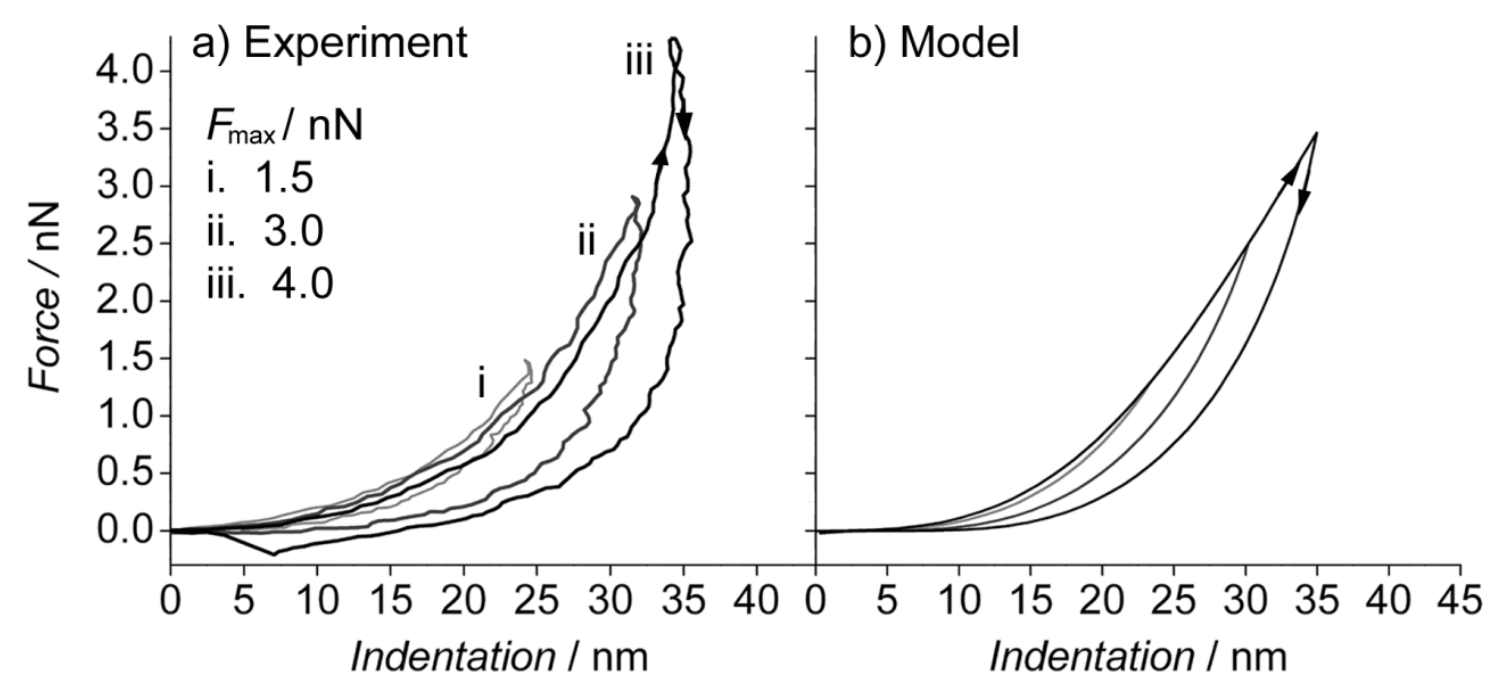

Figure 3.20 Force indentation curves on cross-linked polymer bilayers. (a) Experimental force indentation curves performed with varying maximal indentation force $(1.5-4.0 \mathrm{nN})$. (b) Modeled force indentation curves. Experiments are performed on silicon nitride porous substrates at constant velocity of $2000 \mathrm{~nm} \mathrm{~s}^{-1}$ with a silicon nitride cantilever $\left(k_{m}=1.09 \cdot 10^{-4} \mathrm{Nm}^{-1}, k=0.01 \mathrm{Nm}^{-1}, \eta=0.02 \mathrm{~Pa} \mathrm{~m} \mathrm{~s}, \tau=2 \mathrm{~s}\right.$ where $\left.\tau=\eta / k\right)$.

As it was done for untreated polymer membranes, the force curves performed on the UV-treated polymer membranes were compared with model curves. The theoretical force curves obtained from the modified Kelvin-Voigt model (Figure 3.21b) predict the hysteresis in the cross-linked membranes, however, not to the extent that was observed experimentally. The modeled curves do not reflect the observed hysteresis partly based on the fact that the non-linearity of the indentation curves at high indentation depths is not well represented by the model. In terms of the origin of the hysteresis, is possible that cross-linking of the membrane poly(butadiene) core is incomplete and the interchain interactions due to entanglement are still significant.

To further verify test the cross-linked polymer membrane's resistance, force indentation curves with much higher indentation force $(\sim 16 \mathrm{nN}$ and $\sim 48 \mathrm{nN})$ were performed. In addition to the high indentation force, the AFM tip was left in contact with the membrane for an extended period of time (10 s and $15 \mathrm{~s}$ ) instead of retracting the tip immediately after reaching the preset maximal indentation force. After the prolonged indentation at $16 \mathrm{nN}$ (Figure 3.22a) and at $\sim 48 \mathrm{nN}$ (Figure 3.22b), the membrane was imaged and the line profiles in Figure 3.21c show permanent membrane deformation. 

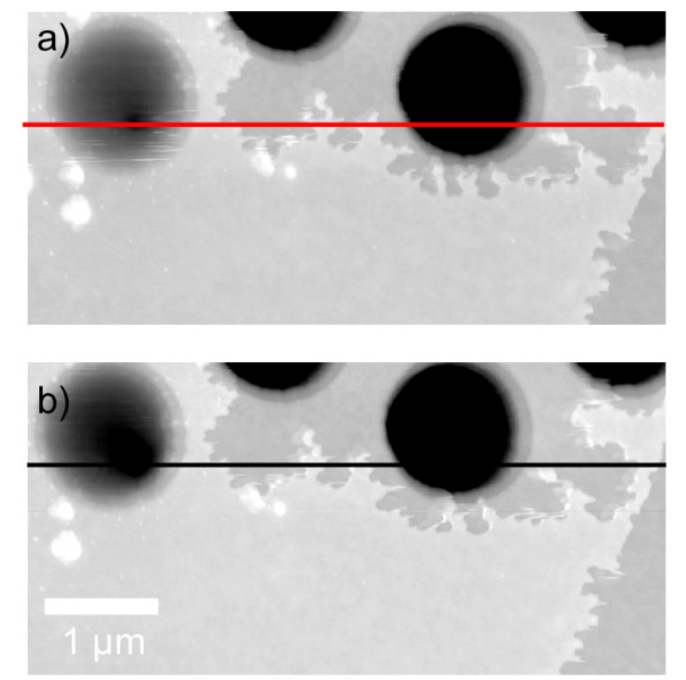

c)

- line profile after $10 \mathrm{~s}$ of $16 \mathrm{nN}$ indentation

- line profile after $15 \mathrm{~s}$ of $48 \mathrm{nN}$ indentation

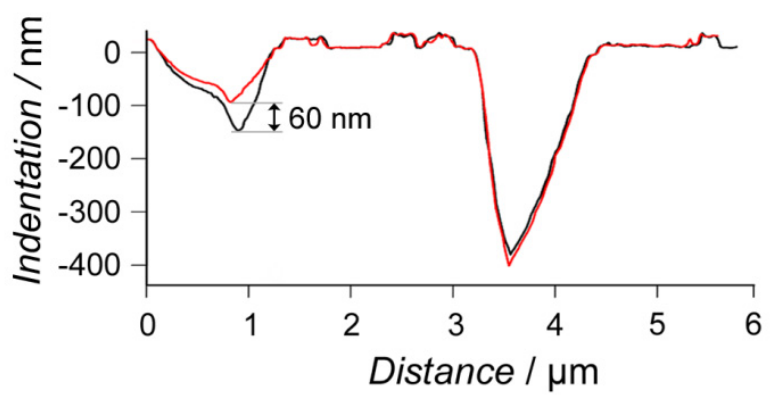

Figure 3.21 Contact mode images of a UV-treated pore-spanning polymer membrane after (a) $10 \mathrm{~s}$ of indentation at $\sim 16 \mathrm{nN}$ of force (red line), (b) $15 \mathrm{~s}$ of indentation at $48 \mathrm{nN}$ of force (black). (c) Line profiles of obtained from a and $b$.

The first conclusion of this high force indentation experiment is that the polymer membrane became significantly more resistant to the applied stress after the UV crosslinking of its poly(butadiene) core. The second observation is that the imprint of the AFM tip was still visible during imaging which indicates that the membrane did not regain its initial topography. In contrast to non UV-treated PB- $b$-PEO membranes, the indentationinduced deformation of cross-linked membranes was either irreversible or the time needed for the membrane to regain its initial topography was beyond the time length of experiment.

\subsubsection{MECHANICAL PROPERTIES OF POLYMER PORE-SPANNING MEMBRANES}

To compare the extent of the hysteresis in all of the experiments, the area enclosed by the indentation and retraction curves was calculated. This area corresponds to the energy dissipation and is quantified in Joules. Comparison between the experimental values of energy dissipation and the values obtained from modeled force curves are shown in Figure 3.23. Notably, a small hysteresis doesn't mean that in turn the viscosity is negligible. In fact, a higher $\eta_{\text {eff }}$ was obtained for cross-linked membranes (Table 3.1). This smaller hysteresis is a result of the immense stiffening of the bilayer. 


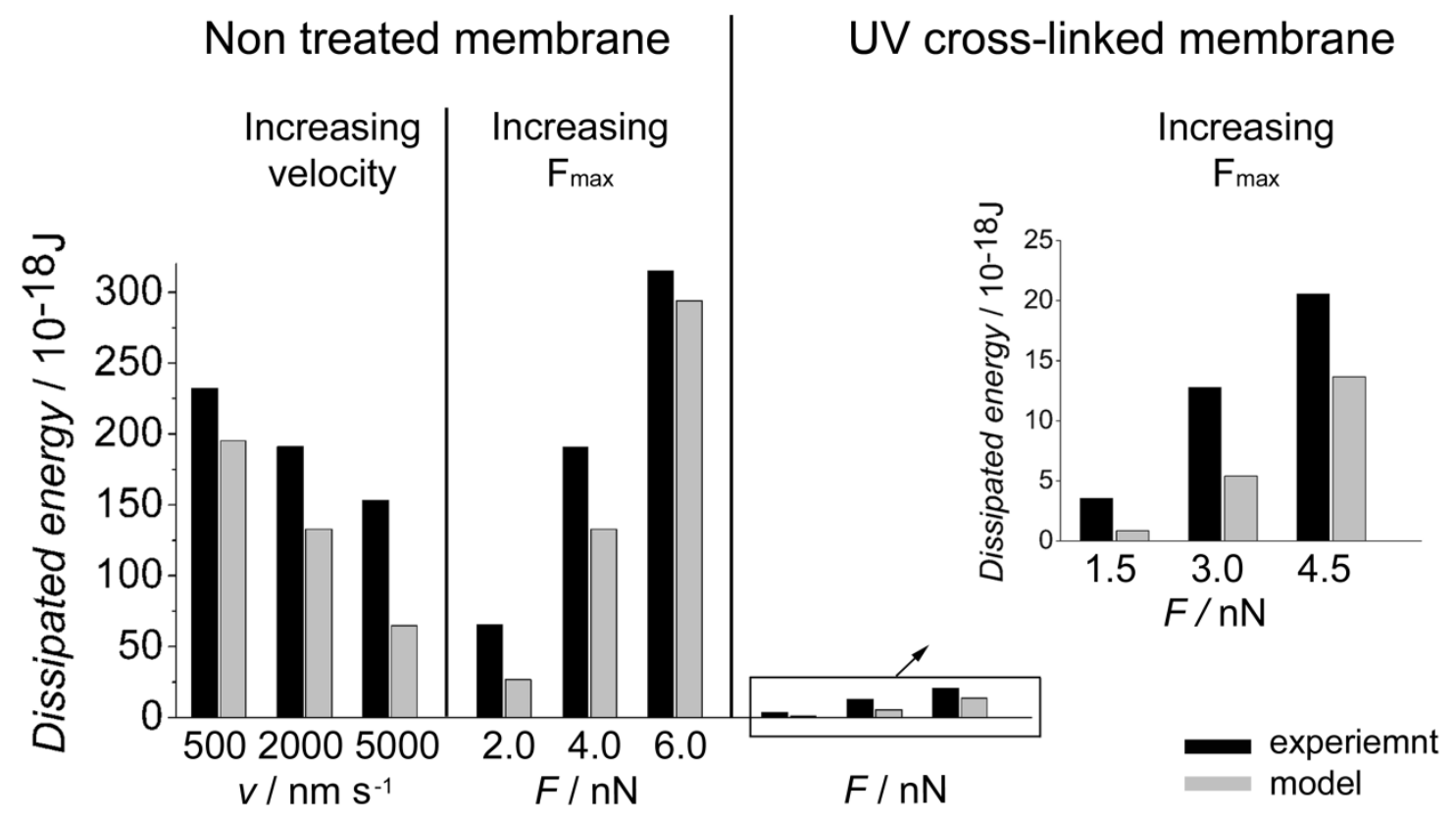

Figure 3.22 Histogram representing the extent of hysteresis observed in the experimental results (black columns) in comparison to the hysteresis predicted by the modified Kelvin-Voigt model (Figure 3.11) (gray columns). The area of the hysteresis represents the loss of energy and is calculated in Joules.

The viscoelastic response of polymer membrane is distinct from that of a phospholipid bilayer. Strongly nonlinear (stretching dominated) force indentation curves were recorded and cannot be explained neither by plate bending nor by pretension alone. Assuming reasonable values for $E$ and $v$, the energy contribution of bending is negligibly small. Pre-tension, however has been shown to produce large apparent spring constants of the bilayer $\left(0.007-0.026 \mathrm{Nm}^{-1}\right)^{11}$ but gives rise to predominantly linear force indentation curves. However, at large forces, stretching dominates over pre-tension. This is in accordance with Lee et al. who found that indentation of graphene layers is mainly governed by a linear combination of stretching and pre-tension. ${ }^{22}$ This linear combination can be interpreted as a parallel combination of a linear spring representing pre-tension and a non-linear cubic contribution from stretching. Fitting of force indentation curve (Figure 3.13) with Eq. 3.9 leads to excellent agreement and reasonable values for the area compressibility in comparison with micropipette suction experiments. The energy loss during indentation becomes apparent in the hysteresis between indentation and retraction and was interpreted in terms of a viscoelastic solid, explaining the observed velocity dependence of the hysteresis and the area compressibility as a function of indentation 
velocity. In contrast to phospholipid bilayers, which display an intrinsic elastic behavior, the entanglement of the long polymer chains and the attachment of the membrane to the substrate give rise to a finite shear viscosity. Evans and Hochmuth ${ }^{20}$ proposed a viscosity of a biological membrane to be in the order of $10^{-6} \mathrm{~Pa} \mathrm{~m}$ s, i.e. more than 3 order of magnitude less than was found for polymer membranes. Dimova et al. found that GUVs derived from PB- $b$-PEO block copolymers also exhibit hyperviscosity paired with low bending rigidity. ${ }^{23}$ In comparison to phospholipid membranes, polymer membranes are more robust and less susceptible to area dilatation - no ruptures events were found in our study. Their low diffusion constant $(<0.0024 \mu \mathrm{m} / \mathrm{s})^{24}$ and higher overall viscosity produces a time-dependant elasticity (viscoelasticity) in which the material is stiff on small time scales and soft on long time scales. This is opposite to deformation experiments on cells where with increasing indentation velocity the hysteresis increases due to the different relaxation times. ${ }^{25}$ In the case of polymer membranes, viscous deformation does not develop on small time scales $t<\tau$ and thus the material behaves elastically. ${ }^{19}$

Table 3.1. Summary of viscoelastic properties of native polymer membranes and UV cross-linked membranes. ${ }^{a}$ A range is given for $E$ and $K_{\mathrm{a}}$ due to their dependence on indentation velocity.

\begin{tabular}{lcccccc}
\hline & $\boldsymbol{k} / \mathbf{N m}^{-1}$ & $\eta_{\text {eff }} / \mathbf{P a ~} \mathbf{~ s}$ & $\tau / \mathbf{s}$ & $\boldsymbol{k}_{\mathrm{m}} / \mathbf{N m}^{-1}$ & $\boldsymbol{E} / \mathbf{P a}$ & $\boldsymbol{K}_{\mathrm{a}} / \mathbf{N m}^{-1}$ \\
\hline Native & 0.015 & 0.005 & 0.333 & $2.63 \cdot 10^{-7}$ & $(1.68-8.65) \cdot 10^{6}$ & $0.030-0.156$ \\
Cross-linked & 0.010 & 0.020 & 2.000 & $1.09 \cdot 10^{-4}$ & $(1.39-8.24) \cdot 10^{9}$ & $25.02-63.78$ \\
\hline
\end{tabular}

As shown in Table 3.1, the Young's modulus increases almost three orders of magnitude from a range of 1.68 to $8.65 \mathrm{MPa}$ to a range of 1.39 to $8.24 \mathrm{GPa}$ upon cross-linking. Cross-linking has a significant impact on the viscosity leading to a nearly 4 fold increase. As a consequence, the relaxation time of the cross-linked polymer membranes rises.

In summary, to perform in-depth force spectroscopy study of pore-spanning membranes, $\mathrm{PB}_{130}-b-\mathrm{PEO}_{66}$ block copolymer vesicles were spread on microstructured porous bottomless silicon nitride substrates and were locally probed by atomic force microscopy revealing their viscoelastic nature. AFM images in conjunction with fluorescence micrographs provided information about the spreading of the vesicles, while the site-specific indentation curves revealed that the free standing bilayers 
withstand lateral dilatation to a large extent. Viscoelastic parameters of polymer membranes were determined on a locally defined area of only a few nanometers using porous substrates. 


\subsection{LIPID MEMBRANES}

\subsubsection{PORE-SPANNING LIPID MODEL MEMBRANES}

The particular architecture of the eukaryotic plasma membrane composed of a fluid lipid bilayer connected to the actin cortex, has been a challenge to model using artificial membrane systems. The focus of the work presented in the following section was to prepare pore-spanning membranes which mimic the characteristics of lipid membranes found in native systems. Plasma membrane is elastically "decoupled" by the associated cytoskeleton, in particular, the actin cortex with a mesh size ranging between 30 and 300 $\mathrm{nm}$, which confines the mechanical properties to these areas. ${ }^{26}$ The actin mesh is the one that allows the plasma membrane to act as a locally heterogeneous elastic shell, which performs various functions pertaining to cell migration, blebbing as well as exo- and endocytosis. $^{27,28}$

Structured silicon nitride $\left(\mathrm{Si}_{3} \mathrm{~N}_{4}\right)$ supports used in this work is reminiscent of the actin mesh of the cell cortex (Figure 3.23), and its use allows formation of both, elastically decoupled free-standing bilayers, and membranes with low lateral tension by modifying the adhesion strength of the bilayer in contact with the pore-rims. In contrast to global (i.e. average) values, spatially restricted local information can be obtained from elastically decoupled free-standing bilayers where the individual pore-spanning bilayers are not influencing each other since they are decoupled by adjacent pore-rims, offering the possibility to map elastic properties with a mesh size given by the interpore distance and pore diameter of the substrate. 

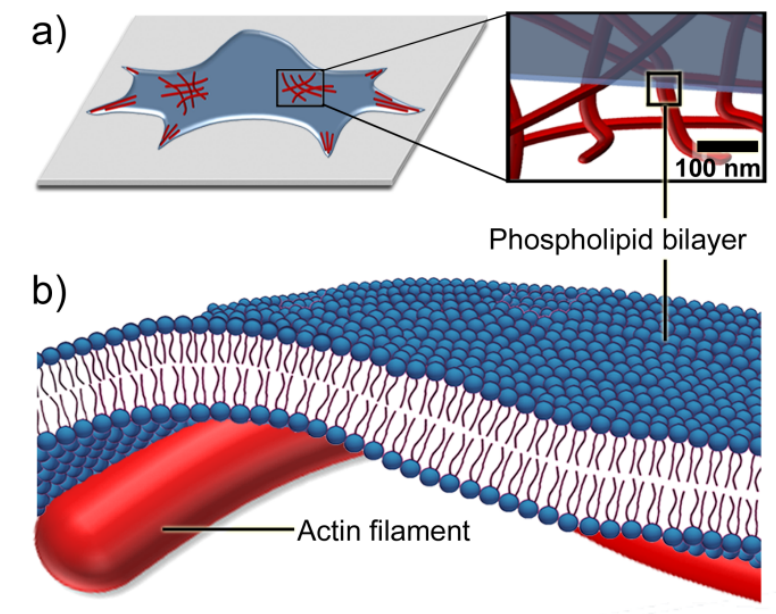

Figure 3.23 Cellular membranes (a) are supported by an intricate scaffolding of the cytoskeleton leading to a laterally tensed membrane, depicted in (b). (c) A pore-spanning membrane patch mimics the tension of a cytoskeleton supported bilayer.

In this present work, non-hybrid, pore-spanning bilayers consisting of POPC, DOPC as well as DOPC/cholesterol mixtures were successfully formed and studied. The porespanning bilayers prepared exhibit low tension $\left(\sigma \leq 3.5 \mathrm{mN} \mathrm{m}^{-1}\right)$ and high lateral mobility $\left(D \cong 8 \mu \mathrm{m}^{2} \mathrm{~s}^{-1}\right)$. These membranes are particularly interesting for biological applications where plasma membrane mimics are necessary for functional reconstitution of proteins that "sense" lateral tension such as mechanosensitive channels.

\subsubsection{PREPARATION OF PORE-SPANNING LIPID MEMBRANES}

\subsubsection{POROUS SUBSTRATE SURFACE FUNCTIONALIZATION}

Preparation of solvent-free, pore-spanning membranes on substrates which have the same surface chemistry on the pore rims as in the pore interior is only possible in certain cases where the interactions between membrane constituents are strong enough to withstand the stress of spanning empty space. Such cases include polymer membranes (as was shown in previous chapter) where the polymers forming the membrane can interdigitate and entangle which confer the membrane the resistance needed to span empty space. In general, however, preparation of native phospholipid pore-spanning membranes on porous surface which has homogenous surface chemistry on the pore rims and inside the pores is difficult. 
Surface modification strategies are routinely employed to deposit lipid monolayers and bilayers on planar as well as porous surfaces. Hydrophobic surface functionalization is used on surfaces to either tether liposomes or to create hybrid pore-spanning lipid membranes by replacing the lower bilayer lipid leaflet of a spreading vesicle by, for instance, a self-assembled alkyl monolayer. ${ }^{29}$ Hydrophilic surface functionalization is used to prepare membranes without replacing the lower leaflet of the bilayer, instead, the membrane rests on a hydration layer present on the hydrophilic surface without delamination. The latter membranes are fluid in two dimensions and closely mimic biological systems.

The system discussed in this work was designed such that the pore-rims of the porous substrate are covered with a hydrophilic surface functionalization which will support a fluid bilayer throughout the sample. This approach allows for the preparation of porespanning membranes lacking much of the unwanted lateral tension created by hydrophobic functionalization. ${ }^{30}$

As a support for the preparation of pore-spanning membranes, silicon nitride $\left(\mathrm{Si}_{3} \mathrm{~N}_{4}\right)$ porous substrates displaying a hexagonal pore arrangement with pore diameters of $1.2 \mu \mathrm{m}$ were used. First, a thin layer of gold was evaporated on the pore-rims and by evaporating a thin $(10-15 \mathrm{~nm})$ gold layer, the gold coverage is restricted predominantly to the porerims. The gold coating procedure was carried out in a thermal evaporation unit and the gold layer thickness was monitored with a quartz film thickness monitor. The gold-coated substrates were exposed to oxygen plasma, followed by argon plasma in order to clean the gold and the $\mathrm{Si}_{3} \mathrm{~N}_{4}$ surfaces. ${ }^{31}$

After plasma treatment of the surfaces, a self-assembled monolayer (SAM) of mercaptoethanol was formed on the gold-coated porous substrate producing moderately hydrophilic pore-rims. Formation of thiolate SAM on a clean gold surface, with $80-90 \%$ coverage, is assumed to happen almost instantaneously after immersion in thiolate solution (concentration of tens of $\mathrm{mM}$ ). Any additional immersion time of the gold surface in the SAM solution allows for thiol rearrangement which results in increased and more uniform SAM coverage of the gold surface. ${ }^{32}$ To verify if the mercaptoethanol SAM formed on the gold surface, contact angle of a buffer drop deposited on a gold-coated glass after incubating it in mercaptoethanol SAM solution was measured. Comparison of 
the contact angle values obtained on mercaptoethanol SAM, gold-coated glass slides which were immersed either in tetradecanethiol solution or pure ethanol is shown in Figure 3.24.

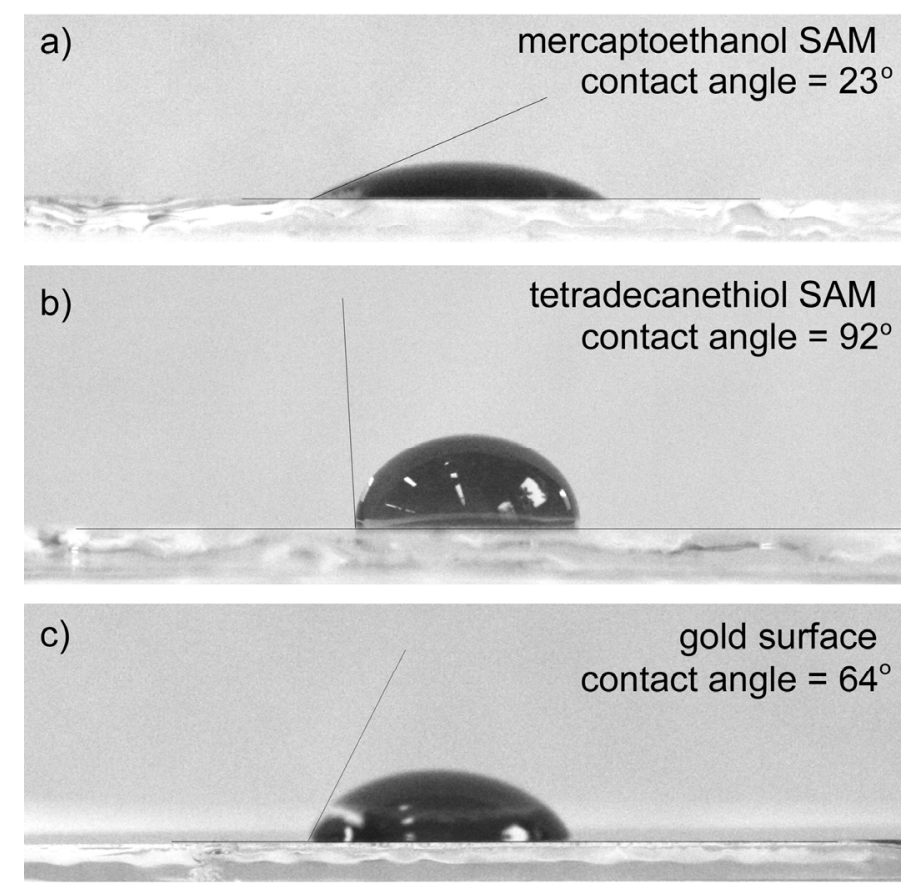

Figure 3.24 Contact angle measurement. To confirm the formation of a self-assembled monolayer (SAM) on gold surface, contact angle of a buffer drop deposited on (a) mercaptoethanol SAM, (b) tertadecanethiol SAM and (c) gold surface immersed in pure ethanol was measured.

The contact angle of $23^{\circ}$ obtained on mercaptoethanol is much lower than that determined on a gold surface which was immersed in pure ethanol $\left(64^{\circ}\right)$, which confirms that the mercaptoethanol confers hydrophilic character to the gold-coated surface. As expected, tetradecanethiol SAM renders surface highly hydrophobic $\left(92^{\circ}\right)$.

The mercaptoethanol hydroxyl-terminated SAM formed on the gold surface is a prerequisite for deformation-induced vesicle rupture of the giant unilamellar vesicles (GUVs) which are subsequently added to the measuring chamber. It has been observed that when GUVs are added to a clean (non modified) $\mathrm{Si}_{3} \mathrm{~N}_{4}$ porous substrate, the vesicles spread, but all the pore-spanning membranes rupture within a few seconds to a few minutes, covering the pore-rim, as well the as the surface of the pore-interior due to the high adhesion energy that disfavors pore-spanning membranes. The hydrophilic $-\mathrm{OH}$ terminated SAM on Au appears to sufficiently decrease the degree to which lipids come into contact with the inner $\mathrm{Si}_{3} \mathrm{~N}_{4}$ surface, so that the phospholipid bilayer interact preferentially with the pore-rims, spreads and avoids contact with the pore interior. Once 
added onto the SAM-functionalized Au-covered porous substrates, the GUVs adsorb onto the surface, flatten and deform until instability-induced rupture occurs and a bilayer patch is formed. Since the $\mathrm{Si}_{3} \mathrm{~N}_{4}$ surface of the inner pore walls is not gold covered and therefore does not support the hydrophilic SAM present on the pore-rims, the bilayer created by GUV rupture does not coat the inside of the pores, therefore leading to a porespanning architecture (Figure 3.25).

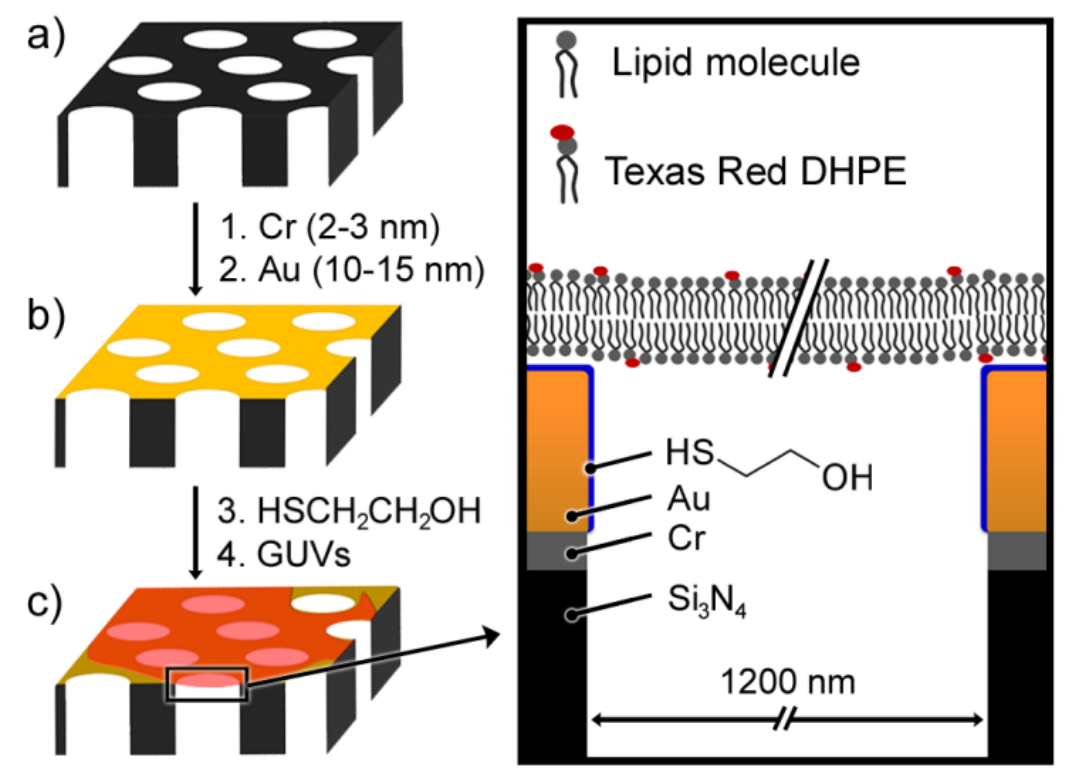

Figure 3.25 (a) Chemical functionalization steps leading to modified porous $\mathrm{Si}_{3} \mathrm{~N}_{4}$ substrates. First, $2-3 \mathrm{~nm}$ of chromium are evaporated, followed by a 10-15 nm gold layer (1-2). (b) The gold-covered surface is then oxygen and argon plasma treated and placed in a $20 \mathrm{mM}$ self-assembly solution of mercaptoethanol (3). (c) Subsequently, the functionalized substrate is placed in PBS, GUVs are immediately added and the membrane patches are formed (4). Inset: enlargement of a functionalized pore-rim showing the lipid bilayer drawn to scale.

Functionalization of the porous substrate is followed by addition of GUVs from which the membrane patches are formed.

\subsubsection{FORMATION OF PORE-SPANNING MEMBRANES}

Pore-spanning membranes are formed from vesicles which rupture and spread on the functionalized porous surface. GUVs used in this study are formed by electroformation technique described in the experimental section. The sizes of the GUVs vary and some of the vesicles enclose smaller ones inside (Figure 3.26). 

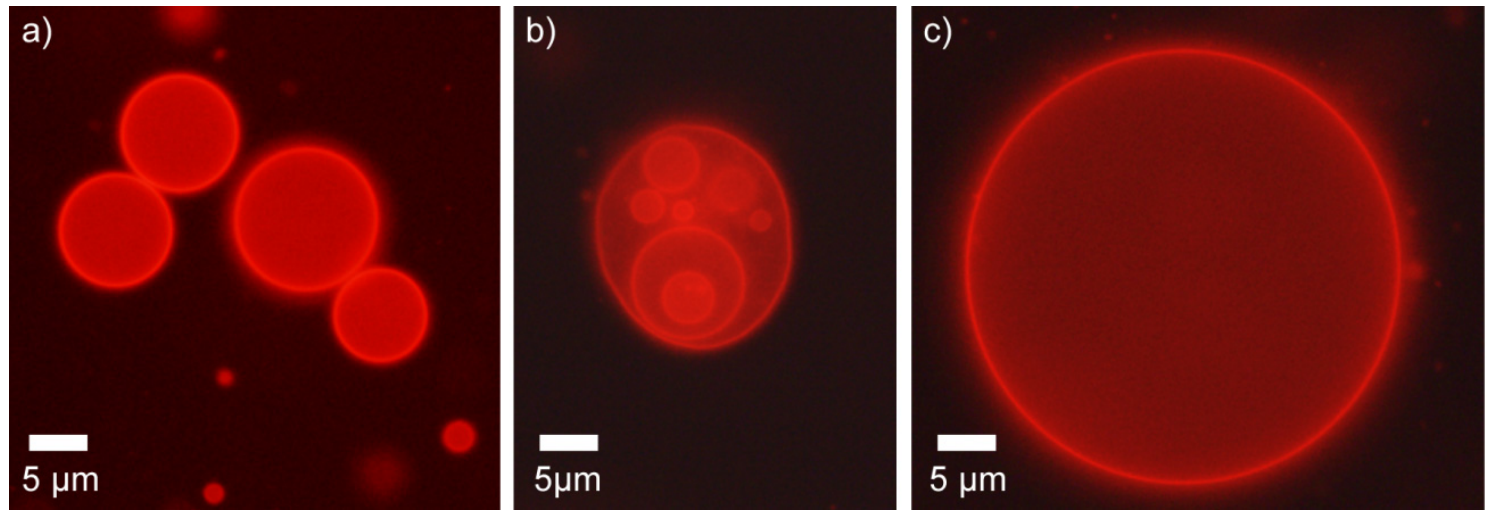

Figure 3.26 Fluorescence images of giant unilamellar POPC vesicles labeled with $0.1 \%$ TexasRed-DHPE used to prepare pore-spanning membranes. (a) 10-15 $\mu \mathrm{m}$ diameter vesicles, (b) a vesicle inside of which smaller vesicles are enclosed and (c) $\sim 50 \mu \mathrm{m}$ giant vesicle

The final size of the membrane patch formed on the porous surface depends, in part, on the size of the vesicle from which the patch is formed. In general, larger GUVs result in larger membrane patches, however, due to the rupture process, not all of the membrane forming the GUV is transferred as a membrane patch onto the porous surface. The process of pore-spanning membrane formation was observed through videmicroscopy (Figure 3.27). Based on the images, the bilayer formation process is envisioned as follows: the higher interior sucrose density of the vesicles in comparison to the surrounding PBS results in vesicle sedimentation to the porous surface. Similarly to the spreading mechanism on $\mathrm{SiO}_{2}$ planar substrates proposed by Hamai et al., the vesicle initially tumbles along the surface until it is close enough to overcome the activation barrier and attaches firmly to the surface. ${ }^{33}$ The increase in adhesion area flattens the sessile liposome until adhesion energy matches the lysis tension leading to vesicle rupture and planar bilayer formation. 
a)

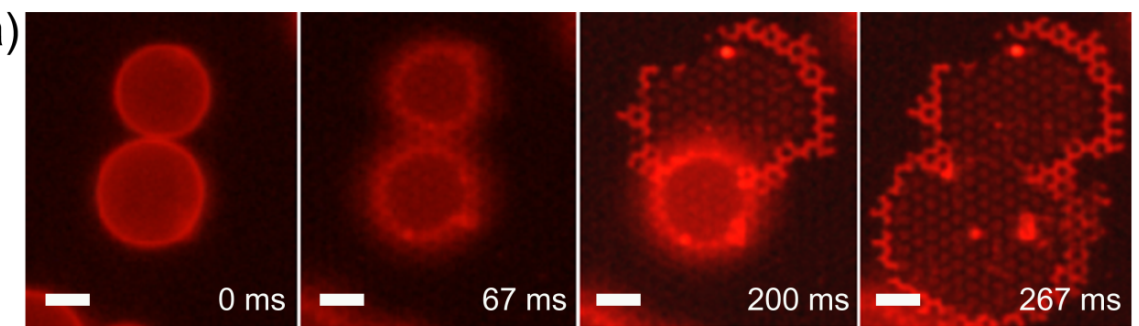

b)

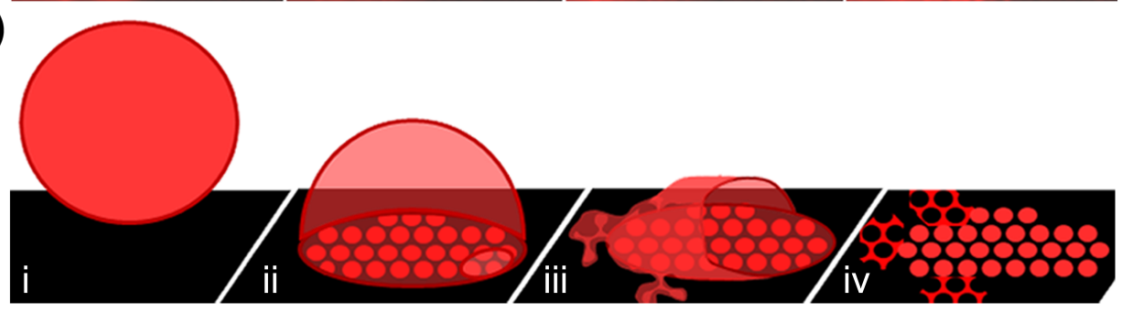

Figure 3.27 (a) Time resolved fluorescence images showing the spreading of GUVs on a mercaptoethanol functionalized porous surface. (b) Schematic representation of the vesicle spreading process: i) vesicle sedimentation on the surface, ii) adhesion, iii) flattening and iv) vesicle rupture and bilayer patch formation (Scale bars: $5 \mu \mathrm{m}$ )

In detail, for strong adhesion, the energetic competition, which determines the conformation of the sessile vesicle, involves the balance between adhesion energy and elastic stretching $\left(K_{\mathrm{a}}\right.$ : area compressibility modulus, approx. $\left.10^{2} \mathrm{~mJ} \mathrm{~m}^{-2}\right)$ of the membrane. If the elastic tension $\left(\sigma=K_{\mathrm{a}} \Delta A / A_{0}\right)$ equals the lysis tension, reached at a relative area change $\Delta A / A_{0}=5 \%$, the vesicle ruptures. ${ }^{34}$ Typically, the size of the final patch (Figure 3.27a, $200 \mathrm{~ms}$ ) is larger than the initial adhesion area of the GUV (Figure $3.27 \mathrm{a}, 67 \mathrm{~ms}$ ) however, the surface area of the pore-spanning bilayer patches are smaller than the total vesicle area. It is conceivable that due to the violence of the rupture process, most of the lipid membrane that is not in proximity to the initial contact area does not deposit as pore-spanning bilayer. This loss of bilayer material is attributed to the violent nature of the spreading mechanism, which also results in a transformation of excess membrane into smaller vesicles upon rupture.

\subsubsection{CHARACTERIZATION OF PORE-SPANNING MEMBRANES}

\subsubsection{PORE-SPANNING LIPID BILAYERS INVESTIGATED BY FLUORESCENCE MICROSCOPY}

Initial characterization of the pore-spanning membranes relies on fluorescence microscopy, however, fluorescence images of lipid membranes differ from fluorescence images of polymer membranes. In contrast to pore-spanning polymer membranes where 
the fluorescence of the entire membrane patch is visible, the fluorescence of porespanning lipid membranes on functionalized substrates is discontinuous. The observed change in fluorescence is related to the porous substrate and not the type of membrane investigated. In the case of pore-spanning polymer membranes, silicon nitride porous substrates are used without any modification. In the case of lipid pore-spanning membranes, surface functionalization is based on the formation of a SAM on gold-coated substrates. It is the gold layer evaporated onto the pore-rims which quenches the fluorescence in its vicinity (up to $10-15 \mathrm{~nm}$ ) ${ }^{35-37}$, which explains the lack of fluorescence on flat membrane covered areas of the functionalized substrate and non-continuous fluorescence on porous area of the membrane-covered substrate. The advantage of the fluorescence quenching is the all-or-none appearance of pore-spanning membranes. Fluorescence image in Figure 3.28 captured various steps of pore-spanning membrane formation.

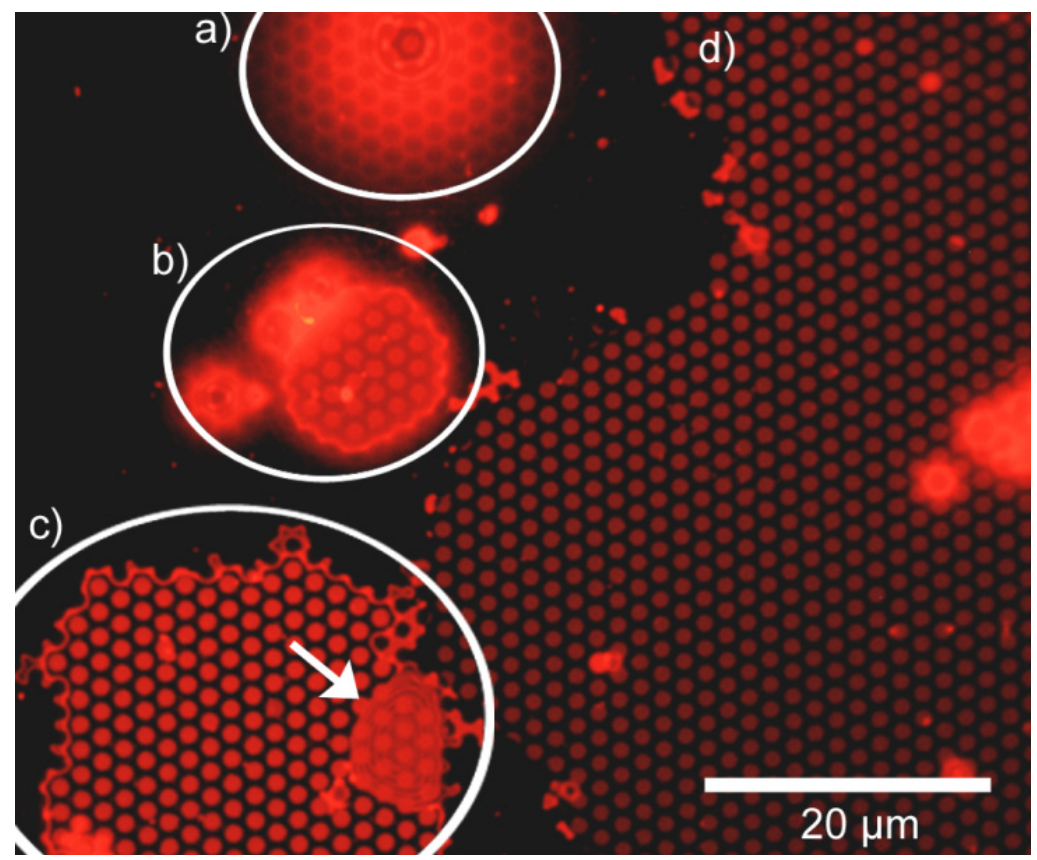

Figure 3.28 The image was captured while focusing at the porous substrate surface. (a) vesicle has made initial contact with the surface, (b) vesicle has partially flattened on the surface, (c) although the membrane patch seems to have formed on the surface, the high intensity fluorescence edges of the "patch", the deflating area (arrow) and the increased fluorescence intensity which is much higher than the intensity of the patch beside indicate that the vesicle has deflated however it has not ruptured. (d) large pore-spanning membrane patch.

Similarly to the work of Hamai et al., two rupture pathways for isolated GUVs were observed. Asymmetric patches (Figure 3.29a) and symmetric (Figure 3.29b) and were 
formed when the rupture initiation site is closer to the side of a vesicle or at the top of the adsorbed vesicle, respectively. ${ }^{33}$

a)

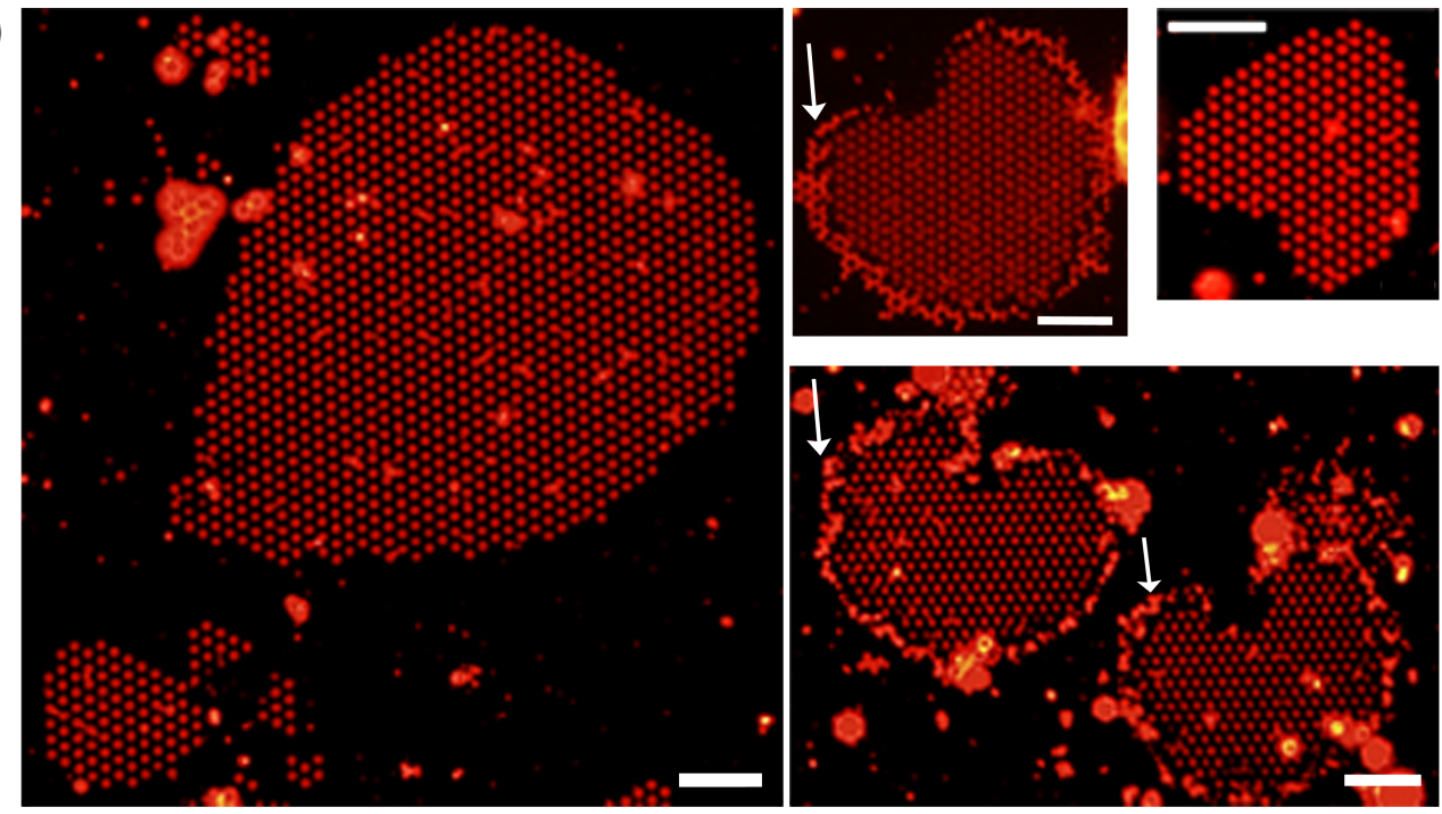

b)
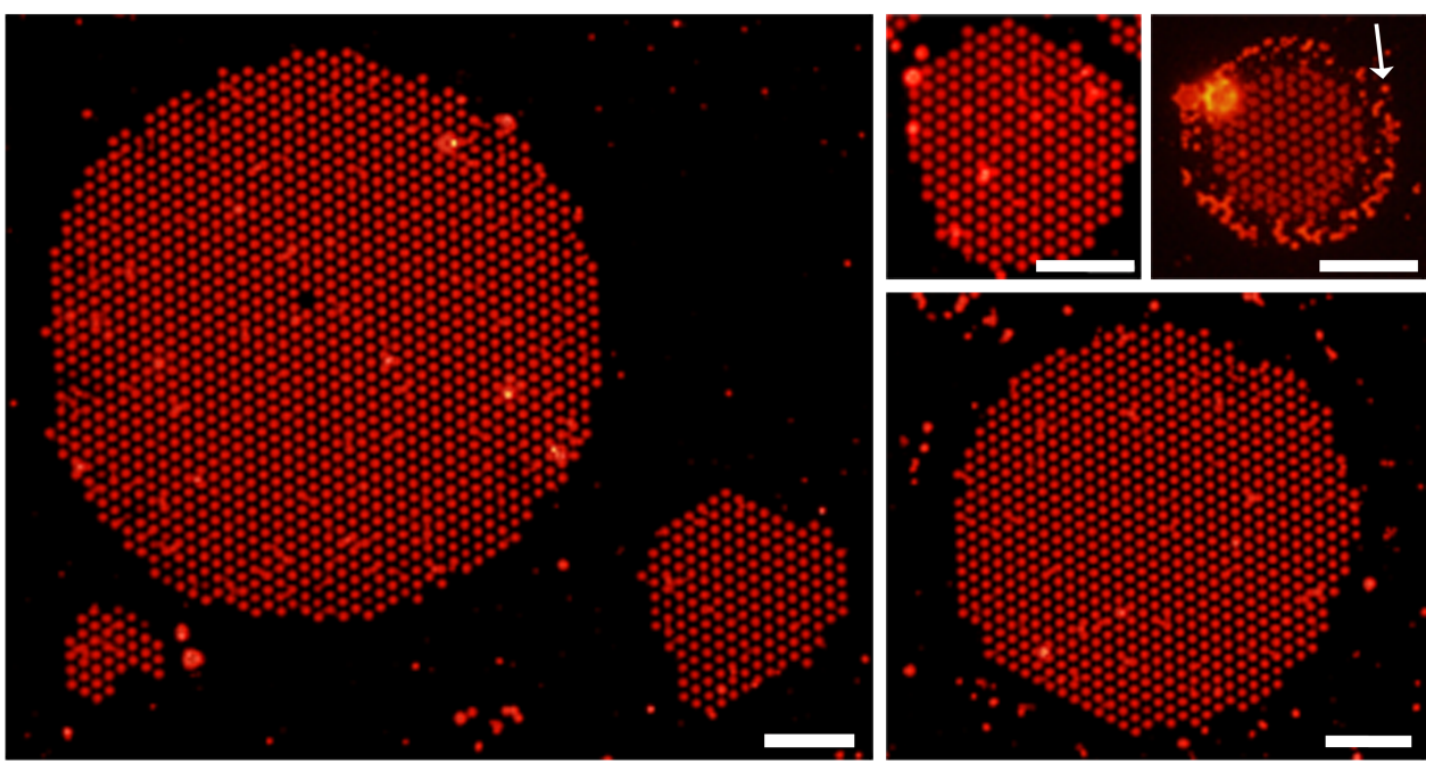

Figure 3.29 (a) asymmetric pore-spanning lipid membrane patches and (b) symmetric pore-spanning membrane patches. When present, non-continuous halo around bilayer patches are marked with an arrow.

The presence of a brighter non-continuous halo around bilayer patches was sometimes observed (see Figure 3.29, arrows). Since the overall appearance on the functionalized porous substrate is governed by the thickness-limited quenching of the fluorescence by the underlying gold film, the higher intensity fluorescence of the material must be related to its thickness in comparison to the single bilayer membrane. 


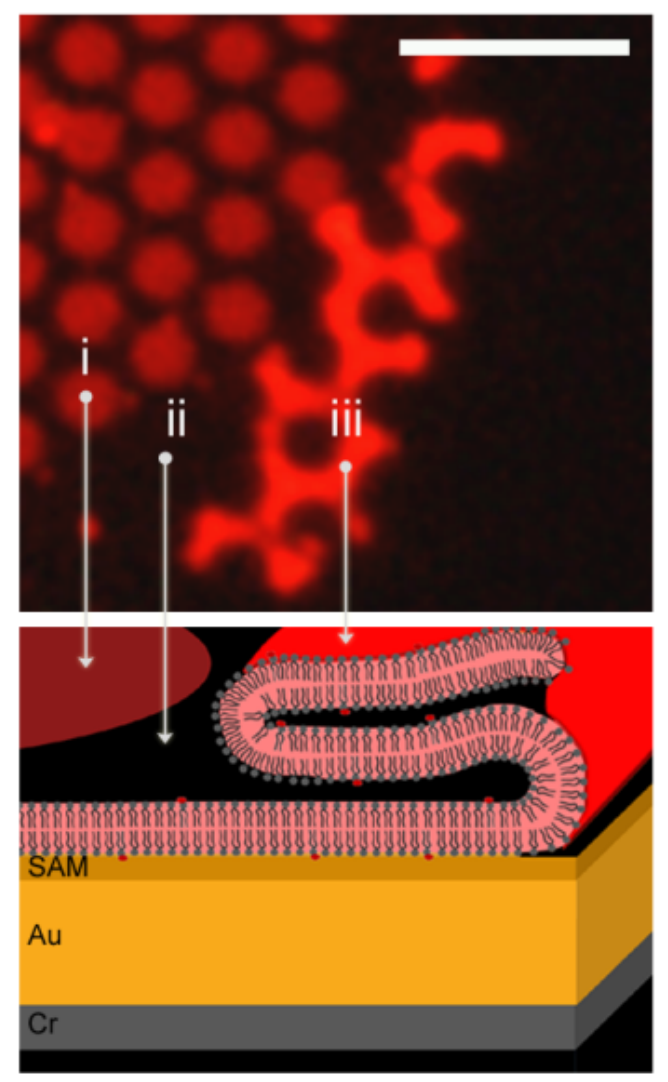

Figure 3.30 Fluorescence image of the filopodia giving rise to excess lipid material on the pore-rims which results in increased fluorescence intensity in comparison to the membrane-spanned pores. (Scale bars: 5 $\mu \mathrm{m})$

The excess lipid material at the outer boundary of the membrane patch that shows up as brightly fluorescent pore-rims, and is attributed to surface enhanced fluorescence originating from fluorophores that are sufficiently far away from the gold-coated porerims (Figure 3.30, area iii). Furthermore, in some cases, the adhesion of the GUV on the pore rims immediately drives the extension of filopodia along the pore rims without suspending the pores. As the GUV deflates but does not rupture, the sessile vesicle becomes surrounded by a network of filopodia as shown in figure 3.31a. When a vesicle comes into contact with the surface and ruptures almost immediately, large filopodia networks are not observed. Instead excess lipid material, which did not span the pores, settles randomly around the membrane patch. In general however, when the surface has been functionalized shortly before GUVs were added, membrane large patches are formed $(>20 \mu \mathrm{m})$ and filopodia formation is limited. 
a)

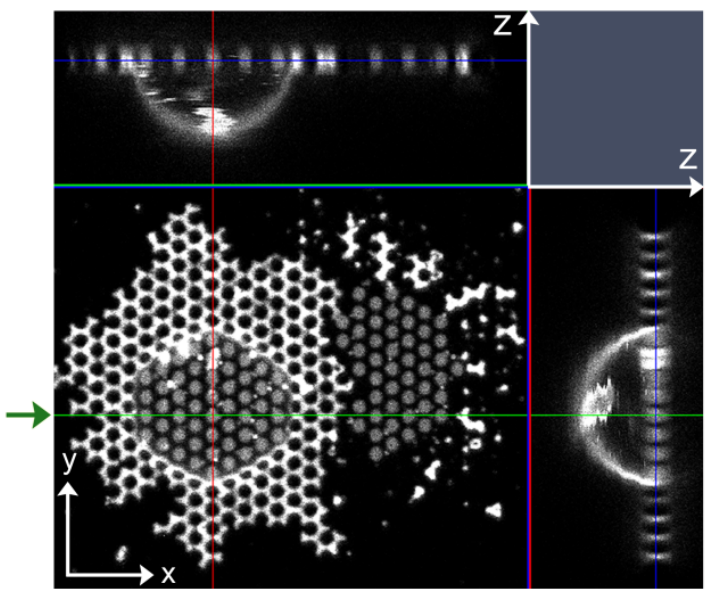

b)

Z

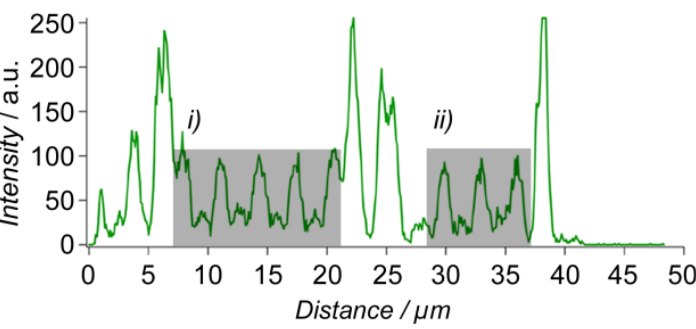

Figure 3.31 In (a), a confocal fluorescence laser scanning microscopy z-stack was used to obtain a 3dimensional image of a mercaptoethanol functionalized substrate where three stages of GUV spreading were captured: a sessile vesicle, pore-rim with excess lipid material and pore-spanning membrane (indicated by crossing lines). The sessile vesicle (i) located to the left of a previously ruptured vesicle (ii) exhibits a dome shape confirmed by the orthogonal cut view (top and right side panels in a). The highly fluorescent pore-rims surrounding the vesicle results from surface enhanced fluorescence originating from filopodia consisting of double-bilayers which possess fluorophores sufficiently far away from the underlying gold film. A patch of pore-spanning membrane is visible on the right side of the vesicle. The fluorescence intensity profile obtained from the line marked with an arrow shows that the fluorescence intensity originating from the lower membrane of the sessile GUV (shaded area i) is identical to that of the pore-spanning membrane (shaded area ii), which confirms that only a single bilayer spans the pores.

Close examination of the single plane CLSM fluorescence image (Figure 3.31a) and the corresponding fluorescence intensity profiles (Figure 3.31b, areas i and ii) provide further information about the thickness of the lipid membrane spanning the pores. The image shows a sessile vesicle (Figure 3.31a, middle) attached and partially flattened on the porous substrate, an already formed bilayer patch beside it formed from another adjacent vesicle and pore-rim areas that have the highest fluorescence intensities of the image. Since the vesicle that is in contact with the porous surface is still intact, the vesicle area spanning the pores must be that of a bilayer membrane. The fluorescence intensity from that sessile GUV (Figure 3.31b, shaded area i) is identical to the fluorescence intensity originating from the pore-spanning patch located on its right side (Figure 3.31b, shaded area ii). This observation leads to the conclusion that, indeed, single lipid bilayers were obtained by the preparation procedure proposed here.

Fluorescence microscopy is an essential tool for localization of pore-spanning lipid membrane patches. However, quantitative information about the bilayer membrane formed is limited. Atomic force microscopy imaging was performed on the porous substrates to further characterize the pore-spanning membranes formed. 


\subsubsection{LATERAL MOBILITY OF PORE-SPANNING MEMBRANES INVESTIGATED BY FRAP}

An important characteristic of native membranes is the lateral mobility of the phospholipids in the 2-dimensional plane of the bilayer. In hybrid membrane systems, where the bottom leaflet of the bilayer is replaced by a hydrophobic monolayer covalently attached to the pore-rims, the membrane is anchored and the diffusion of the lipids is limited to the top lipid leaflet of the bilayer. This anchoring at the pore-rims significantly decreases the overall lipid diffusion constant and alters the appearance of the fluorescence recovery curve of fluorescence recovery after photobleaching (FRAP) experiments.

In the pore-spanning preparation method discussed here, the pore-rims are hydrophilic and the bilayer lipids should diffuse with diffusion coefficients characteristic of bilayers on planar hydrophilic surface. FRAP experiments (Figure 3.32) revealed an average diffusion coefficient of $8 \pm 4 \mu \mathrm{m}^{2} \mathrm{~s}^{-1}$, confirming that the observed pore-spanning bilayers are fluid.

a)

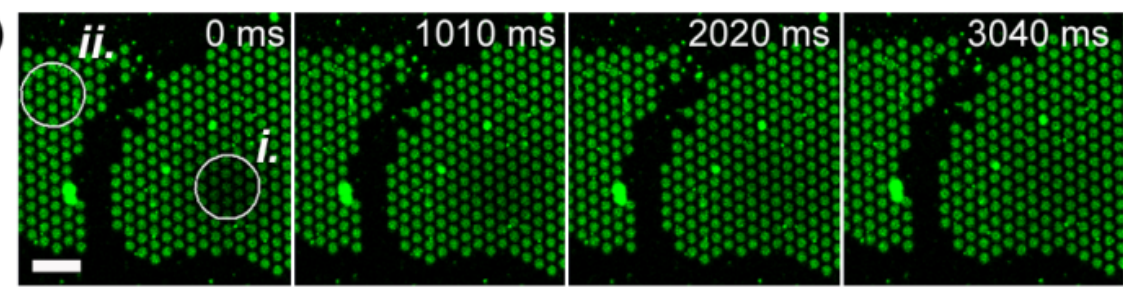

b)

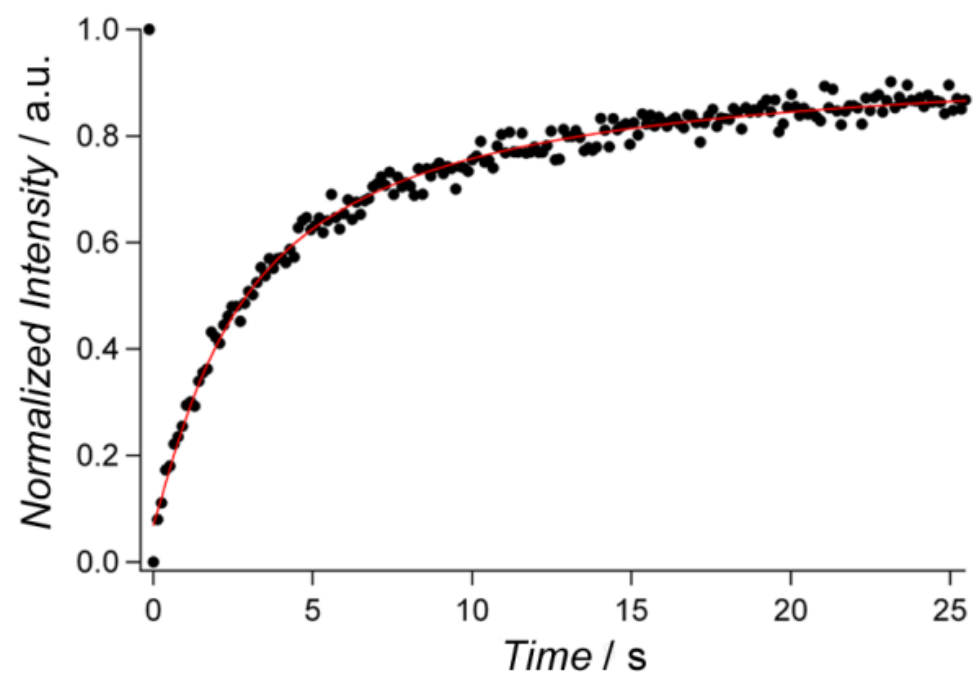

Figure 3.32 Fluorescence recovery after photobleaching (FRAP) was performed on a pore-spanning POPC bilayer labeled with $0.1 \mathrm{~mol} \%$ Bodipy-DHPE. (a) The fluorescence recovery of a bleached area (i) was recorded simultaneously to the reference area (ii). (b) A fit, according to the theory of Axelrod ${ }^{38}$, to the normalized fluorescence recovery curve provides a diffusion constant of $8 \pm 4 \mu^{2} \mathrm{~s}^{-1}$. (Scale bar: $5 \mu \mathrm{m}$ ) 
The immobile fraction of the lipids is $<5 \%$, which indicates that the membrane is not strongly coupled to the substrate. It is argued that a thin hydration layer between the membrane and the hydroxylated, hydrophilic pore-rim surface allows the lipids in both, top and bottom leaflets, to diffuse freely. Based on the average diffusion constant found in this system, which is close to what can be found for unconstrained lipid bilayer, it can be concluded that the coupling of the bilayers to the pore-rims does not significantly reduce the mobility of the lipids. ${ }^{39-41}$ Diffusion constants $D$ of various model membranes have been measured and reported in the literature with GUVs $D=1.1-6.3 \mu \mathrm{m}^{2} \mathrm{~s}^{-1}{ }^{42}$ BLMs exhibiting very high lateral mobility due to the presence of solvent $D=20.6 \pm 0.9$ $\mu \mathrm{m}^{2} \mathrm{~s}^{-1},{ }^{43}$ solid-supported membranes (on glass) $D=1-4 \mu \mathrm{m}^{2} \mathrm{~s}^{-1}{ }^{44}$, polymer-cushioned membranes are less mobile $D=0.88-1.13 \mu \mathrm{m}^{2} \mathrm{~s}^{-1}$, polymer tethered membranes are even less fluid $D=0.5-0.89 \mu \mathrm{m}^{2} \mathrm{~s}^{-1} .{ }^{45}$ Solvent containing hybrid membranes on porous substrates $\left(7 \mu \mathrm{m}\right.$ diameter) are highly mobile such as BLMs $D=14 \mu \mathrm{m}^{2} \mathrm{~s}^{-1}{ }^{46}$, while solvent-free, hybrid membranes on AAO (60 nm diameter) close to the values found for GUVs $D=7 \pm 3 \mu \mathrm{m}^{2} \mathrm{~s}^{-1} 47$. Based on the determined diffusion constant, which resembles that of highly mobile lipids in giant liposomes ${ }^{48,49}$, the lipids of the pore-spanning membranes prepared here seem to be unconstrained by the pore-rims. In addition, this finding is also supported in the shape of the recovery curve which perfectly follows the theory of Axelrod. Derivations from the ideal behavior are expected for a heterogeneous system, where two distinctly different diffusion constants would be observed. ${ }^{46}$

\subsubsection{PORE-SPANNING MEMBRANES STUDIED BY AFM IMAGING}

The presence and location of pore-spanning lipid membrane patches were determined by fluorescence microscopy and the corresponding areas were imaged by AFM. Determination of membrane thickness is straightforward when the membrane is imaged on a flat surface. However, due to the gold film fluorescence quenching up to $10-15 \mathrm{~nm}$ in thickness, single bilayer membranes are not detected (by fluorescence) on flat surfaces. To circumvent this limitation, flat areas around pore-spanning membrane patches were imaged in order to obtain membrane covered flat surface (Figure 3.33). 
a)

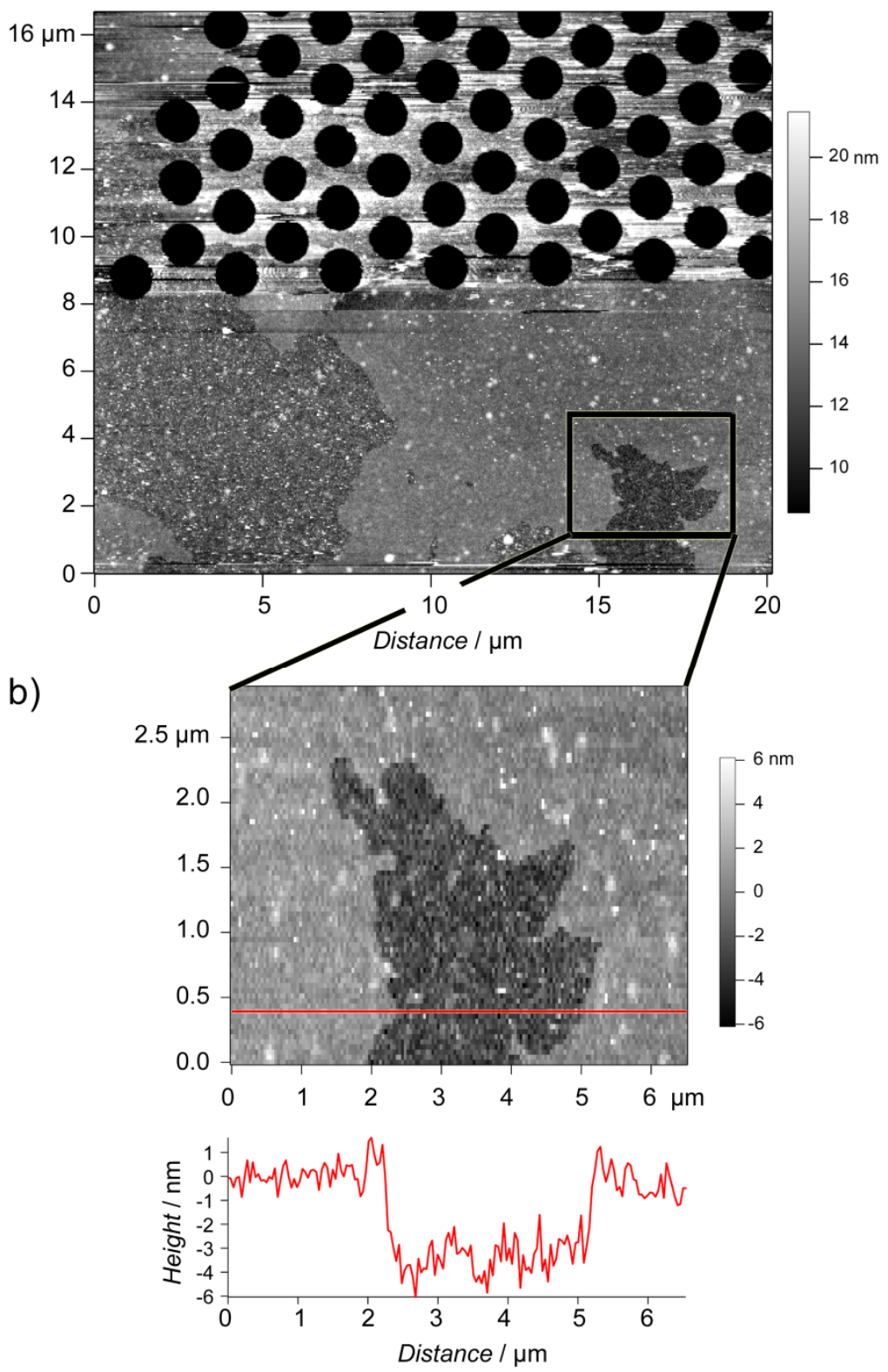

Figure 3.33 (a) Contact mode AFM image of a bilayer membrane patch at the boundary of flat and porous area of the substrate. The membrane is visible on the flat part of the substrate. (b) An imperfection in the bilayer membrane was used to measure its thickness. The height difference of 4-5 nm corresponds to a single bilayer thickness confirming the presence of a single bilayer membrane on the substrate.

Line profile obtained from the AFM contact mode image of the membrane covered flat area of the substrate show membrane thickness of $4-5 \mathrm{~nm}$ which is expected for a single bilayer. In addition to verifying single bilayer thickness of the membrane patches crated on the functionalized porous surfaces, correlation between fluorescence microscopy and AFM images provides confirmation about the pore-spanning nature of the membranes (Figure 3.34). 
a)

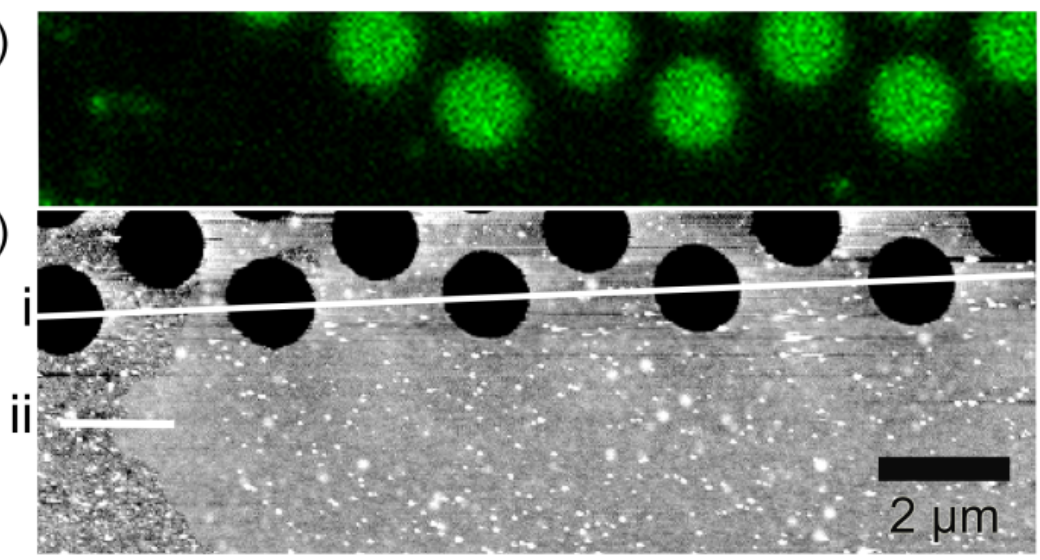

c)
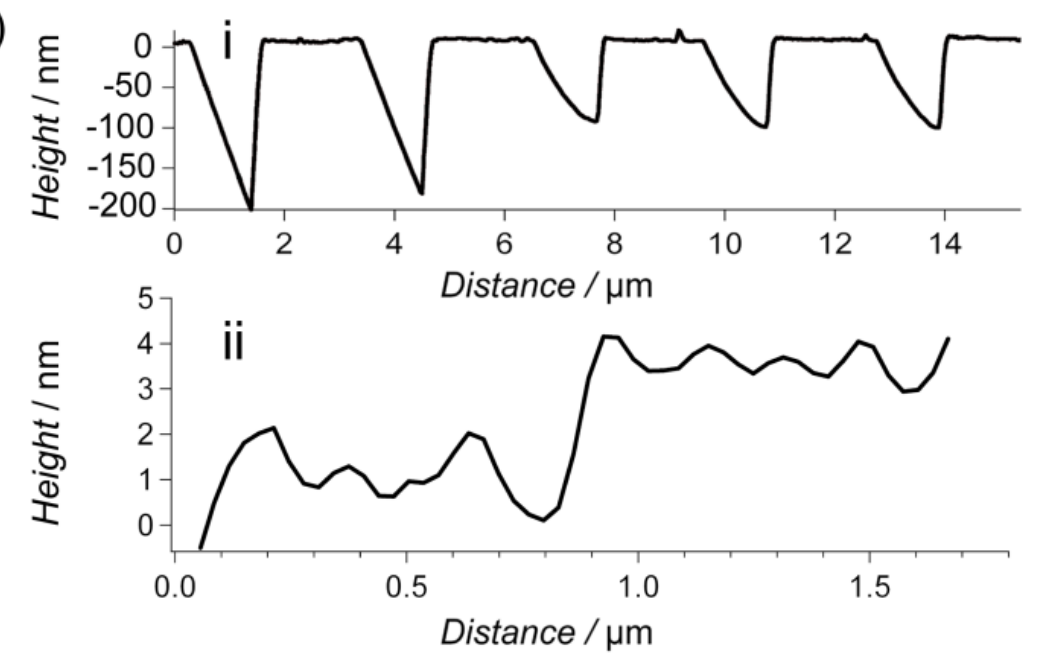

Figure 3.34 (a) Correlation between a CLSM fluorescence image of a pore-spanning membrane and (b) AFM image of the same area. (c) Cross-sections from AFM image show (i) membrane-spanned pores where 3 out of 5 pores are covered and (ii) the presence of a single bilayer membrane $(\sim 4 \mathrm{~nm}$ height difference).

Only pores that show fluorescence (Figure 3.34a) display a reduced penetration depth during AFM imaging (Figure 3.34b and 3.34c, line profile i) since they are covered with a membrane. Membrane thickness is obtained from the line profile acquired on the flat part of the sample (Figures 3.34b and 3.34c, line profile ii) and a thickness of $4-5 \mathrm{~nm}$ is found which is indicative of a single bilayer.

AFM imaging was also used to investigate the high intensity fluorescence excess lipid material found at the edges of some pore-spanning membranes (Figure 3.35). 


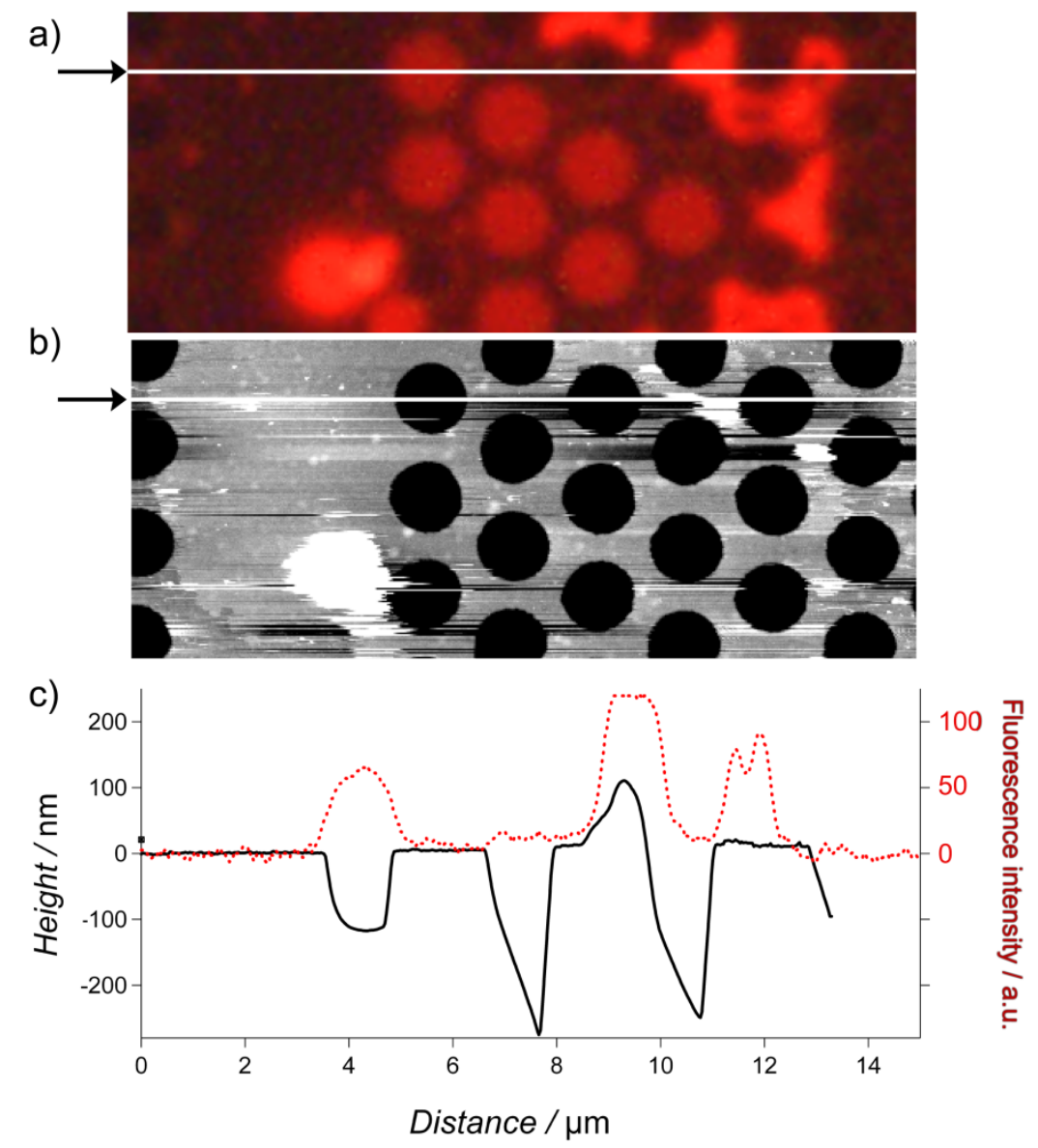

Figure 3.35 (a) Fluorescence image showing membrane-covered pores and excess lipid material on the pore rims. (b) Contact mode AFM image of the same areas as shown in (a). (c) Line profile of the fluorescence intensity (dotted line) coincides with the line profile obtained from the AFM image (solid line) indicating that the increased fluorescence originates from excess lipid material (height $\sim 100 \mathrm{~nm}$ ) present on the pore-rims.

A pore-spanning membrane patch surrounded by areas of increased fluorescence intensity was located by fluorescence microscopy (Figure 3.35a) and imaged by AFM (Figure 3.35b). Line profiles taken from both the fluorescence image and the AFM (Figure 3.35c), show that the increased fluorescence intensity corresponds to excess lipid material found on the surface. Although most of the excess lipid material was displaced during AFM imaging, some remained and its thickness was measured to be $\sim 100 \mathrm{~nm}$. The line profile obtained from that AFM image is compared with the fluorescence intensity profile (Figure 3.35c) and the increased fluorescence intensity corresponds to the high features imaged by AFM. The high thickness value is well above the 4-5 $\mathrm{nm}$ value found for single bilayer membranes and is also beyond the 15-20 $\mathrm{nm}$ material thickness under which the fluorescence is quenched by the underlying gold. It is therefore 
appropriate to define the highly fluorescent areas around the lipid patches as excess lipid material that is originating from the violent GUV rupture.

\subsubsection{AFM FORCE SPECTROSCOPY OF PORE-SPANNING MEMBRANES}

Characterization of the pore-spanning lipid membranes was completed by performing force indentation experiments. The goal of this type of characterization was to determine the position of the pore-spanning membrane with respect to the top of the porous substrate. In the case of hybrid membranes, where the lower leaflet of the bilayer is replaced by a hydrophobic SAM, the membrane lines the entire gold surface where the SAM is present which includes some of the pore interior. This results in invagination of the membrane which tends to maximize the interactions between the SAM and hydrophobic fatty acid chains of the phospholipids (Figure 3.36a). In the case of hydrophilic surface functionalization presented in this work, the vesicles rupture without delaminating resulting in membrane patches where the bilayer lays atop the pore surface, supported by a thin layer of water (Figure 3.36b).

a)

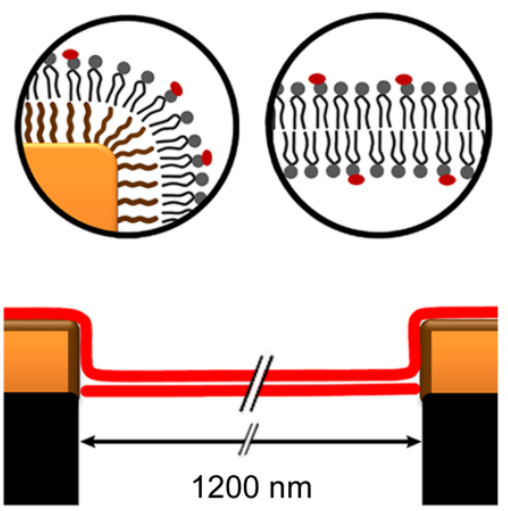

b)
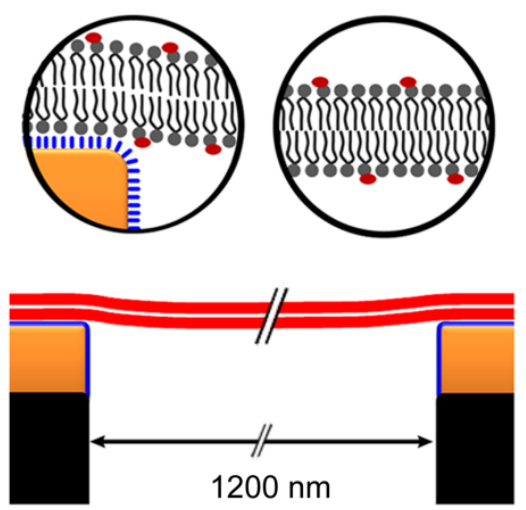

Figure 3.36 Schematic illustration showing the difference between (a) pre-stressed and (b) low tension pore-spanning bilayer lipid bilayers.

In order to verify the topography of the pore-spanning membranes, force volume imaging was performed on the pore-spanning bilayers. A force volume image is obtained by producing maps of force-indentation curves on the surface (Figure 3.37). Each pixel of the image represents the distance travelled by the AFM tip before reaching its preset force. In order to reach a given force, the AFM tip travels less when the pore is covered by a membrane in comparison to an uncovered pore. Therefore, covered pores are represented by lighter areas, while darker ones correspond to uncovered pores. 
a)

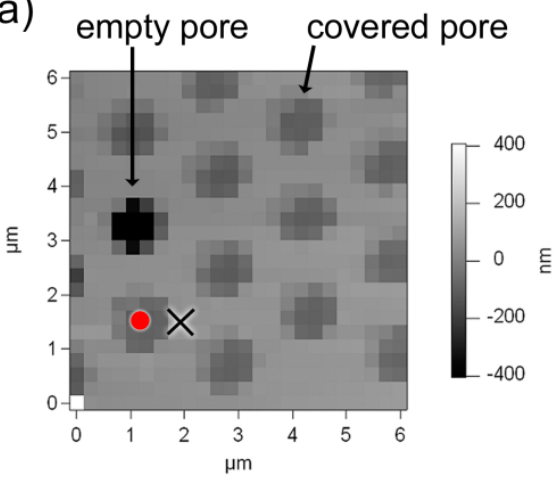

b)

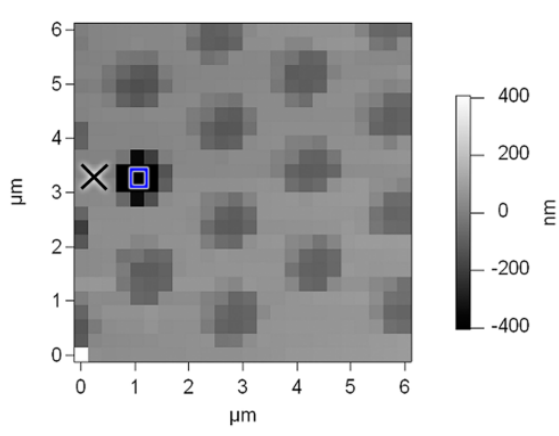

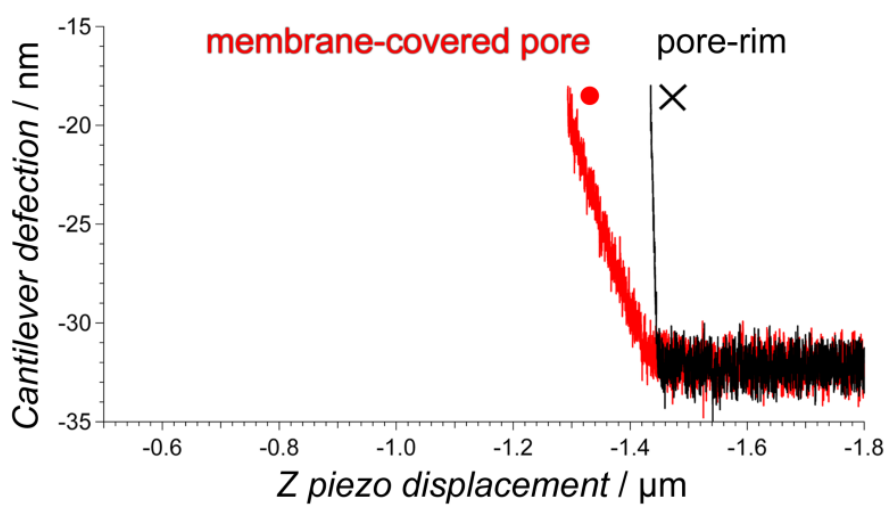

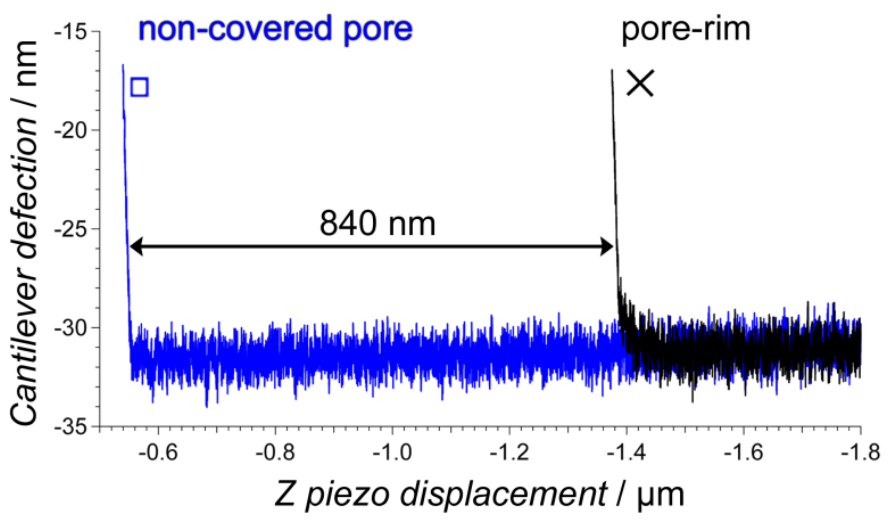

Figure 3.37 (a) Force volume AFM image of a porous surface where only one pore is not covered with a membrane. Force indentation curves performed on a pore-rim $(x)$ and a pore-spanning membrane $(\bullet)$ are shown beside the image. (b) Comparison between indentation curves performed on a pore-rim ( $\mathrm{x}$ ) and an empty pore $(\square)$. In contrast to the empty pore where the tip must travel $840 \mathrm{~nm}$ before reaching a predetermined setpoint, the contact point is identical in the case of flat surface and membrane-covered pore indicating that there is no membrane invagination into the pore.

After acquiring force volume images, force indentation curves performed on either a membrane-covered pore or a non-covered pore were compared with a reference indentation curve performed on the pore-rim. In the case of a membrane-spanned pore, the contact point of the AFM tip with the membrane was identical to the position of the adjacent pore-rim. In contrast, the AFM tip had to travel $840 \mathrm{~nm}$ in comparison to the reference pore-rim before it reached the same setpoint. According to this finding, the bilayer membrane seems to span the pores without lining their insides or protruding. (Figure 3.37a, curves with symbols $\mathrm{x}$ and $\bullet$ ). The behavior of the pore-spanning membranes prepared in this work contrasts that of hybrid nano-BLMs, deposited on hydrophobic self-assembled monolayer, which line the gold coating inside the pores, thus covering the entire gold surface to gain the maximal adhesion energy regardless of the imposed curvature stress. ${ }^{30}$ It is an indication that the adhesion of bilayers spread on mercaptoethanol functionalized porous substrates is significantly weaker than on hydrophobic SAMs. In conclusion, the vesicle spreading process of GUVs on hydrophilic 
$\mathrm{OH}$-terminated mercaptoethanol functionalized porous substrates is very similar to what has been observed previously on flat $\mathrm{SiO}_{2}$ surfaces. ${ }^{50}$

In summary, characterization of the pore-spanning bilayer membranes prepared according to the new protocol presented in this work was carried out by fluorescence imaging and atomic force microscopy. Fluorescence microscopy provided initial confirmation about the pore-spanning character of the membranes as well as the location of the pore-spanning membrane patches. FRAP experiments were performed to quantify lateral mobility of the membranes spanned over the pores and CLSM fluorescence microscopy provided insight into the single bilayer nature of the membrane. AFM imaging confirmed that the membranes are pore-spanning and, by providing material thickness values, validated that single bilayer membrane are formed during GUV rupture on the functionalized porous substrates. Furthermore, AFM force indentation curves were used to establish that the pore-spanning membranes are covering the pores without being invaginated inside the pores (as it is the case in hybrid nano-BLMs) nor do they bulge out (which is sometimes observed in the case of solvent-containing membranes). In light of the results obtained during characterization, the pore-spanning membrane preparation procedure presented in this work yields single bilayer pore-spanning membranes patches which are robust and stable enough to undergo AFM force spectroscopy study.

\subsubsection{FORCE SPECTROSCOPY STUDY OF PORE-SPANNING LIPID MEMBRANES}

\subsubsection{COMPOSITION DEPENDANT LATERAL TENSION}

To investigate the mechanical stability as well as the tension of the pore-spanning membranes in detail, site-specific force indentation experiments were performed. The membrane patches were first identified and located by fluorescence microscopy and subsequently probed by force spectroscopy. A typical force indentation curve performed in the middle of a membrane-covered pore is shown in figure 3.38. 


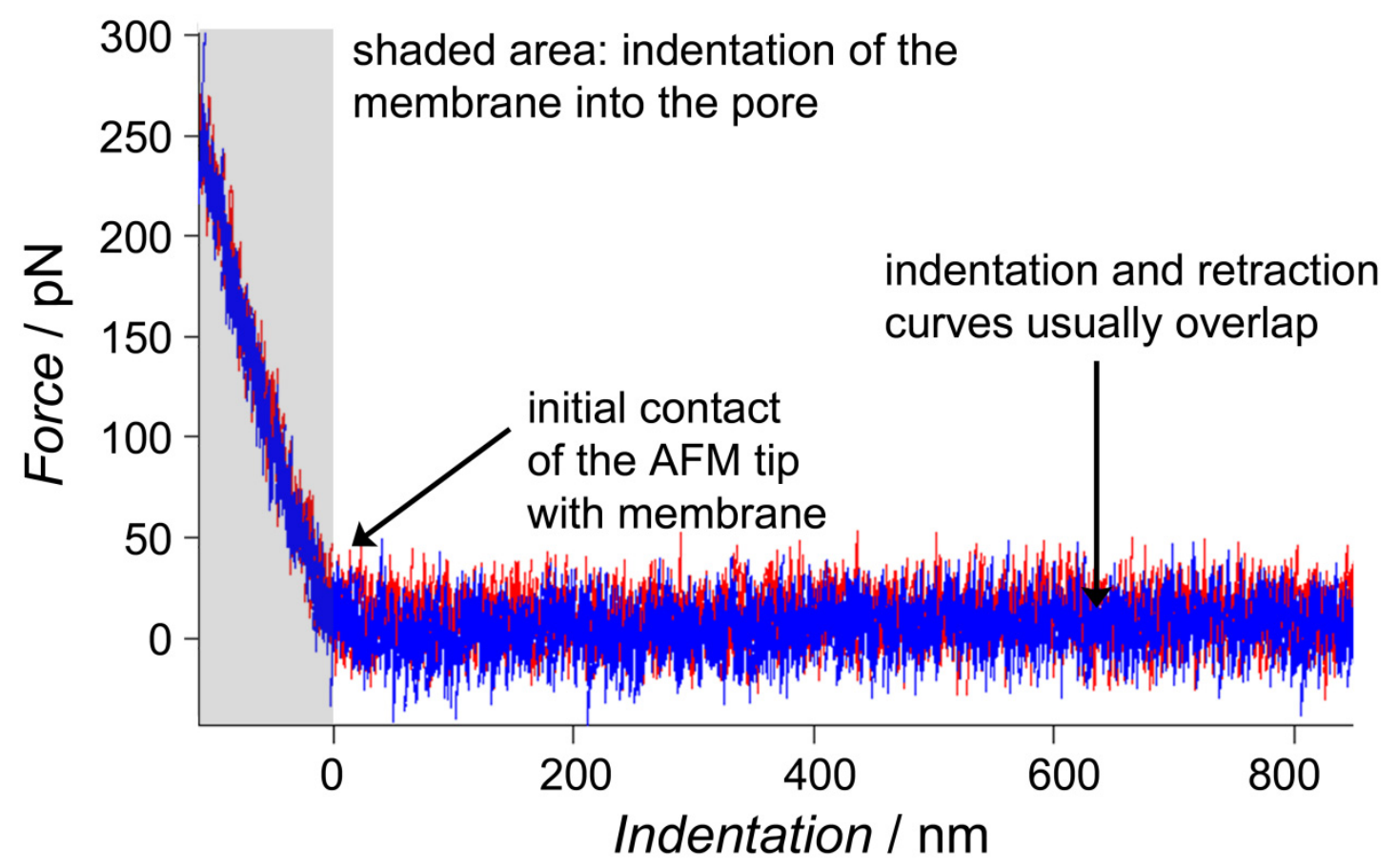

Figure 3.38 Representative force indentation curve performed on a pore-spanning POPC membrane at 2000 $\mathrm{nm} \mathrm{s}^{-1}$ velocity and $250 \mathrm{pN}$ setpoint.

Indentation force curves were performed in the middle of the membrane-spanned pores to reduce the possibility of the AFM tip contact with the interior of the pore and, most importantly, because the membrane indentation theory is valid only when the membrane is indented in the middle. In fact, a control indentation experiment was performed where a membrane-spanned pore was probed along its diameter to determine at which distance, from the pore center, the membrane response changes. AFM images of the pore-spanning membranes, the force indenation curves, $k_{\text {app }}$ obtained from the curves and the dependence of $k_{\text {app }}$ on the indentation position are shown in figure 3.39. 
a)

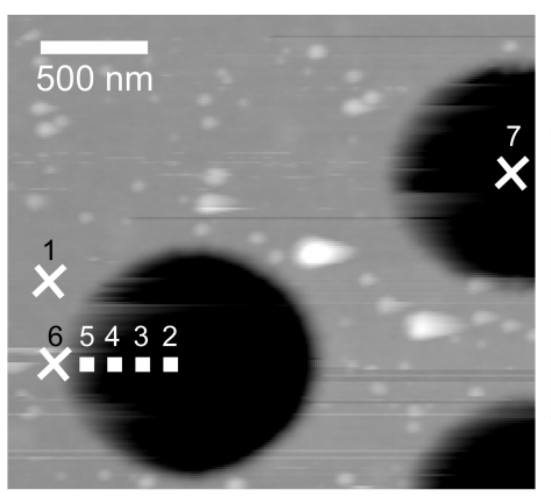

\section{c)}

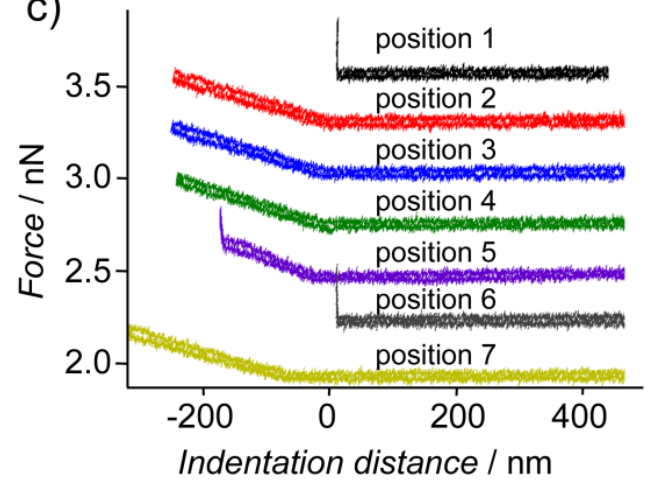

b)

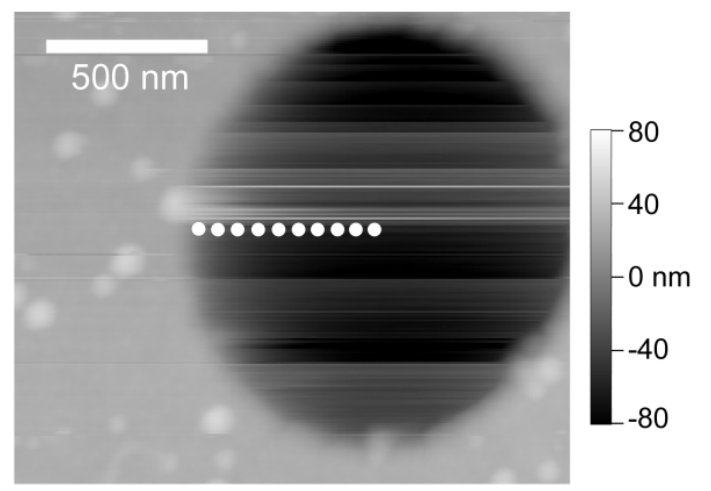

d)

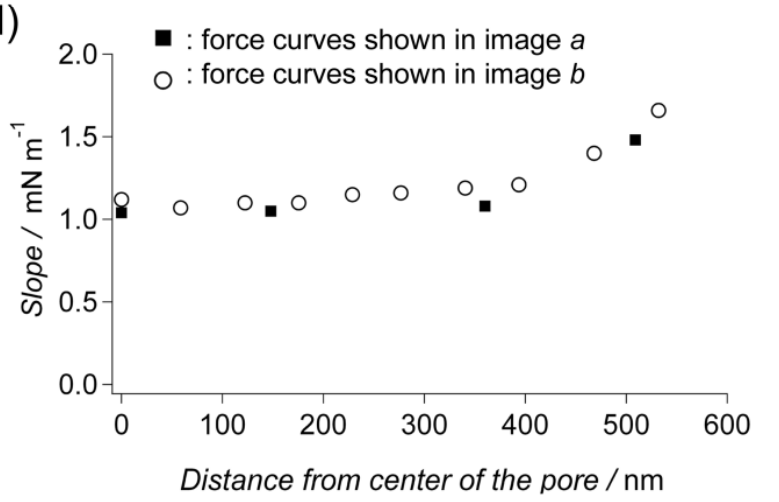

Figure 3.39 Effect of indentation position away from pore center on the response of the pore-spanning membrane. (a-b) AFM images showing the position of where indentation curves were acquired, (c) force indentation curves obtained from positions shown in (a), (d) graph showing the change in the slope $\left(k_{\text {app }}\right)$ with respect to the position of the indentation.

According to the result, the slope is not affected by the position of the indentation up to $300 \mathrm{~nm}$ away from the pore center. When the force curve was performed very close to the side of the pore ( $>300 \mathrm{~nm}$ from pore center), $k_{\text {app }}$ increased and the range of the indentation depth was decreased, due to the hindrance of the pore side walls. This kind of experimental result can be attributed to membrane pre-stress or tension-controlled mechanical response. To prevent the any artifacts due to tip contact with the pore walls, AFM images of the pore-spanning membrane were acquired so that the exact position of the pore was known and the middle of the pore could be indented.

Altogether, three different lipid compositions (POPC, DOPC, DOPC/Chol (7:3)) were investigated by force indentation curves. For all these types of membranes, the force indentation curves are linear and do not show a hysteresis upon relaxation, which indicates the absence of any viscous losses during mechanical load. ${ }^{51}$ Rupture events are not observed. The slopes of the force indentation curves $\left(k_{\text {app }}\right)$ were determined and compiled into histograms (Figure 3.40). 


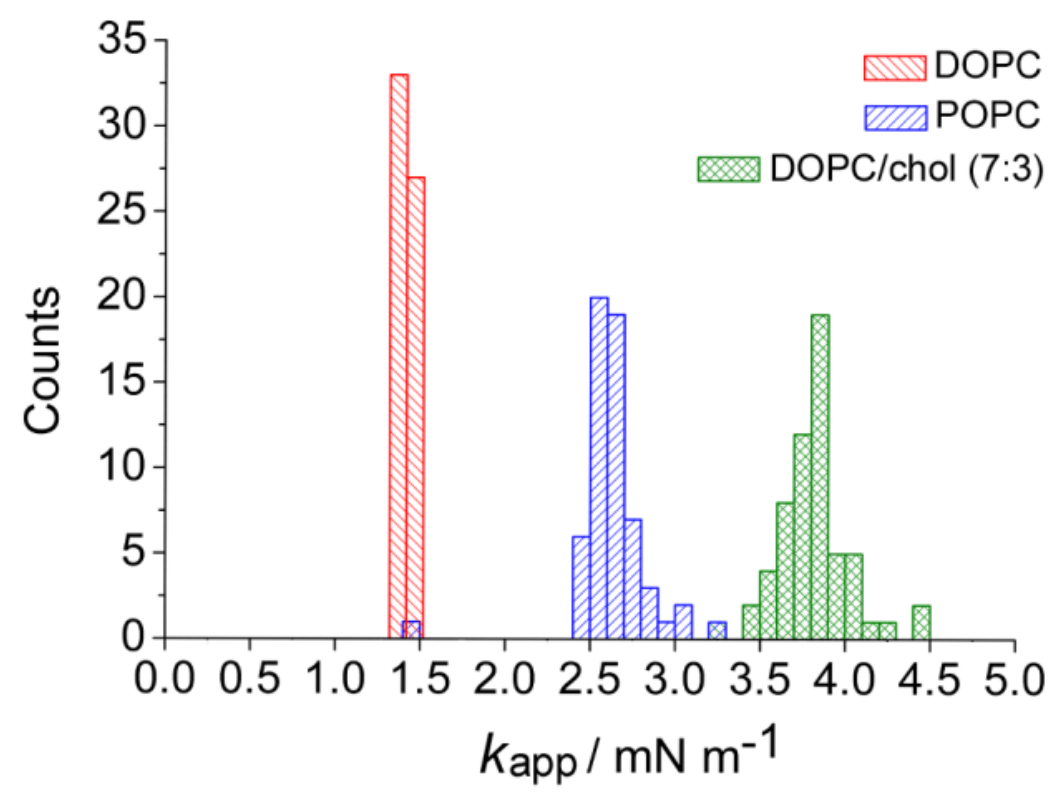

Figure 3.40 Histograms showing the $k_{\text {app }}$ of the pore-spanning membrane patches prepared from various lipid composition GUVs where $k_{\text {app }}$ DOPC: $1.42 \pm 0.02 \mathrm{mN} \mathrm{m}^{-1}, k_{\text {app }}$ POPC: $2.60 \pm 0.12 \mathrm{mN} \mathrm{m}^{-1}$ and $k_{\text {app }}$ DOPC/cholesterol (7:3): $3.82 \pm 0.16 \mathrm{mN} \mathrm{m}^{-1}$.

The membrane tension was obtained by adapting the parameters from the previously developed theoretical framework to the experimental force indentation curves. ${ }^{15,30,52}$ In brief, a bending modulus ${ }^{53}$ of $0.5 \times 10^{-19} \mathrm{~J}$ was assumed for pure DOPC and POPC and $1 \times 10^{-19} \mathrm{~J}$ for the DOPC/Chol (7:3) mixture, which resulted in lateral tensions of $1.0 \mathrm{mN}$ $\mathrm{m}^{-1}\left(1.018 \pm 0.014 \mathrm{mN} \mathrm{m}^{-1}\right)$ for pure DOPC, $2.0 \mathrm{mN} \mathrm{m}^{-1}\left(2.00 \pm 0.09 \mathrm{mN} \mathrm{m}^{-1}\right)$ for POPC, and $3.5 \mathrm{mN} \mathrm{m}^{-1}\left(3.50 \pm 0.15 \mathrm{mN} \mathrm{m}^{-1}\right)$ for DOPC/Chol $(7: 3)$. Based on the theoretical framework recently established by Steltenkamp et al. and Norouzi et al. employing the small gradient approximation of the Hamiltonian, a simple approach was used to convert the experimental force curves into tension values. ${ }^{15,52}$ To calculate lateral tension $\sigma$ from $k_{\text {app }}$ obtained experimentally, force indentation curve (plotted: indentation versus force) was fit with equation 3.10, assuming the absence of binding resistance.

$$
F(x)=\frac{x}{4 \pi \sigma}\left[1-\ln \left(\frac{x R_{\text {tip }}}{2 \pi \sigma R_{\text {pore }}^{2}}\right)\right]
$$

where $F(x)$ represents the indentation depth, $x$ the force, $R_{\text {tip }}$ and $R_{\text {pore }}$ the radii of the AFM tip and the pore, respectively.

It was determined that the mechanical response of the pore-spanning bilayer to indentation of an AFM tip is mainly governed by lateral tension and, to a lesser extent, influenced by bending or stretching of the membrane. In fact, the contribution from 
bending to the mechanical response of the indented bilayer is negligible due to the large pore size, therefore reducing the theoretical effort tremendously. ${ }^{15}$ The resistance to bending is inversely proportional to the square of the pore radius and is 2 orders of magnitude smaller than the measured forces as detailed in Mey et al. ${ }^{30}$ The bilayer basically "flows" into the pore and the resistance to this flow produces a lateral tension. This is supported by the fact that rupture upon indentation is never observed.

Moreover, in order to compare the pore-spanning membranes studied in this work with hybrid nano-BLMs, preparation of hybrid POPC nano-BLMs was attempted, unsuccessfully. It is probable that due to the delamination of the vesicles once they came into contact with the hydrophobic SAM of the interpore surface and the high tension lead to immediate rupture of the pore-spanning bilayers. DPhPC membranes turned out to be more stable, probably due to the interdigitation of methyl groups displayed by the acyl chain. Hybrid DPhPC nano-BLMs were successfully prepared and investigated by indentation experiments. Comparison between the force indentation curves of the two types of membranes is shown in figure 3.41.
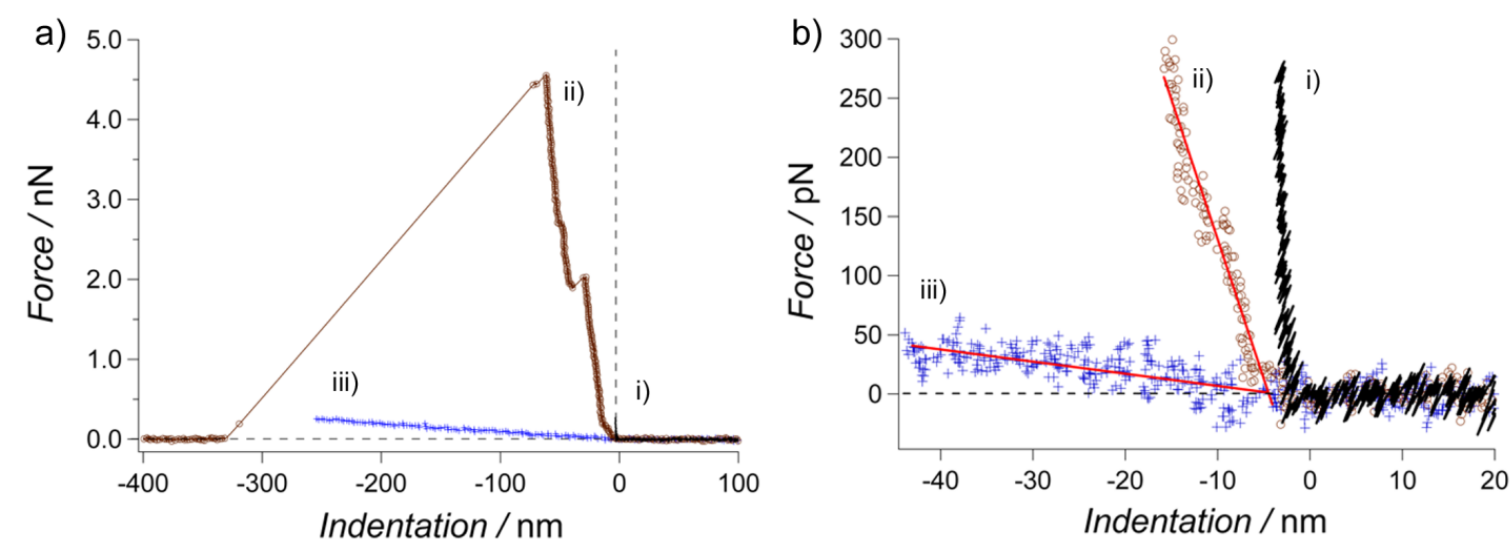

Figure 3.41 Comparison between two types of pore-spanning lipid membranes: 1) low tension membranes on hydrophilic $\mathrm{OH}$ terminated self-assembly monolayers on gold or 2) pre-stressed hybrid membranes on hydrophobic alkyl SAMs. (a) Force indentation curves performed on the (i) pore-rim, on a (ii) $\mathrm{DPhPC} /$ tetradecanethiol hybrid membrane (nano-BLM) and on a (iii) POPC membrane prepared according to the newly introduced protocol. (b) Enlargement of the indentation curves together with the corresponding linear regression to determine the slope $k_{\text {app }}$ : (ii) $20.4 \pm 0.2 \mathrm{mN} \mathrm{m}^{-1}$ and (iii) $1.0 \pm 0.4 \mathrm{mN} \mathrm{m}^{-1}$.

For nano-BLMs composed of DPhPC immobilized on tetradecanethiol, $k_{\text {app }}$ of $20.4 \mathrm{mN}$ $\mathrm{m}^{-1}$ was found (Figure $3.41 \mathrm{~b}$, ii). In contrast, the slope obtained from force indentation curves performed on POPC membrane patches, formed from GUV spreading on mercaptoethanol is about 20 times smaller, $1.0 \pm 0.4 \mathrm{mN} \mathrm{m}^{-1}$, indicative of lower membrane tension (Figure $3.41 \mathrm{~b}$, iii). The physical origin of pre-tension and the lateral 
tension, which is the resistance against area increase upon indentation, is in both cases the preferred adhesion of the bilayer onto the pore rims. Stretching of the bilayer is only possible up to $5 \%$ area increase and gives rise to a cubic dependence of the force on the indentation depth which was not observed. In summary, the mechanical response originates mainly from adhesion of the bilayer to the pore rim, which allows for the determination of the lateral tension from mechanical indentation experiments.

The findings for the different lipid compositions imply that cholesterol not only increases membrane stiffness, i.e. the bending modulus, but also affects adhesion of the bilayers to the hydroxyl-displaying pore-rims. The force indentation method is so sensitive that even tension differences between pure POPC and DOPC are distinguishable with high accuracy. The decreased lateral tension of the presented pore-spanning membranes, in comparison to previously reported ones, is attributed to the reduced adhesion of the bilayer to the pore-rims. This reduced adhesion is achieved by a moderately hydrophilic surface functionalization, which provides a means to mimic the aspect of lateral tension in cellular membranes as it is produced by the cytoskeleton.

\subsubsection{FORCE VOLUME EXPERIMENTS ON PORE-SPANNING MEMBRANES}

Prior to further investigating of the mechanical properties of pore-spanning membranes, control experiments were performed to determine if force volume imaging could be performed on the membranes. The advantage of force volume experiments over isolated force indentation curves is the generation of an image representative of the mechanical response. Furthermore, efficiency of the experiment is increased as a defined area, instead of individual points, is probed. Control experiments consisted in acquiring force volume images and analyzing the force indentation curves in order to determine if the average $k_{\text {app }}$ obtained is comparable with $k_{\text {app }}$ extracted from individual indentation curves. After imaging a pore-spanning membrane, force indentation curves were performed by choosing the middle of each probed pore. Afterwards, a force volume image of the same area was created (Figure 3.42). 
a)

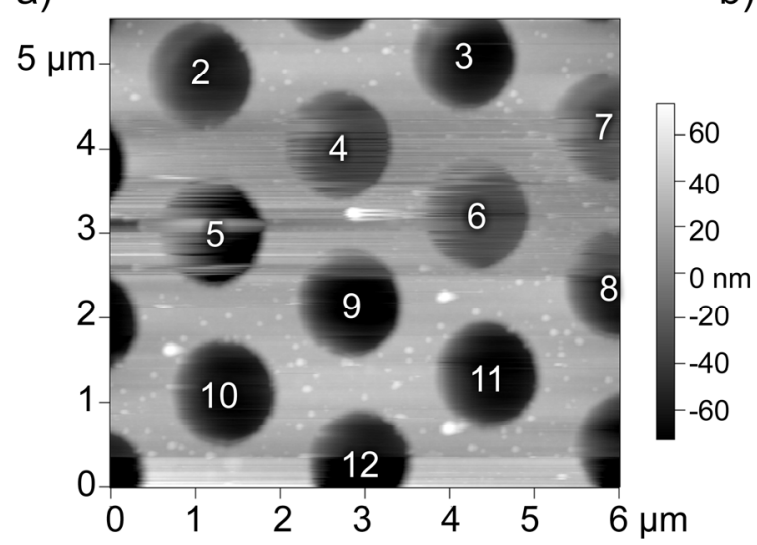

b)

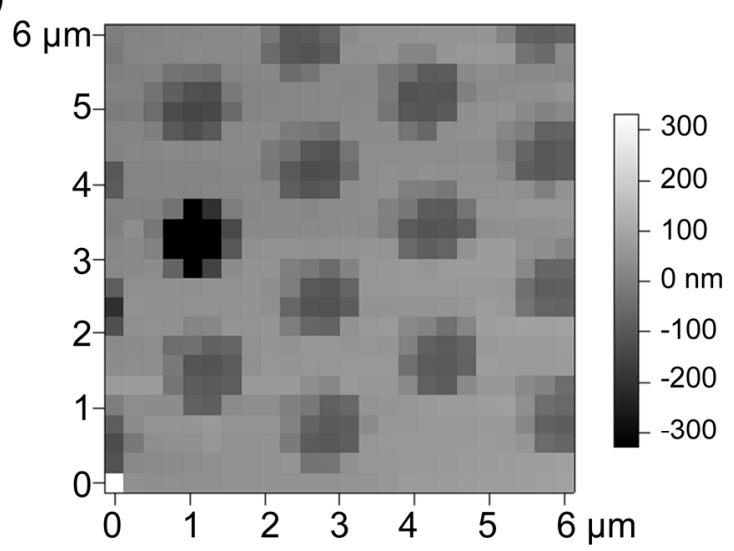

Figure 3.42 (a) AFM image where force curves were performed by choosing the pore center and (b) force volume image of the same area as shown in (a).

In the case of the individual force indentation curves, each pore was probed 5 times before moving to the next pore. The $k_{\text {app }}$ calculated from the force curves is shown in the figure 3.43 .

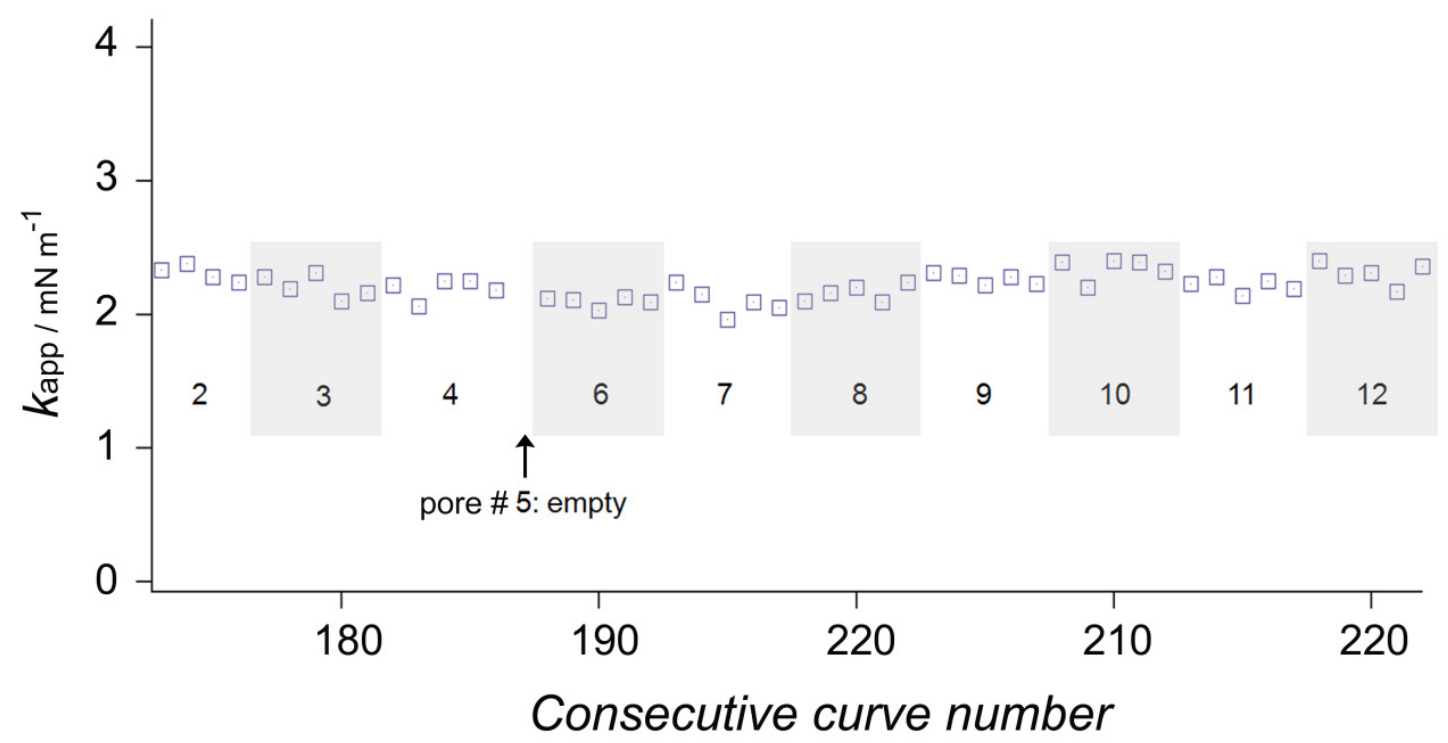

Figure 3.43 Variation of $k_{\text {app }}$ depending on the pore probed.

Variation of $k_{\text {app }}$ values depending on the pore probed did not seem to follow any particular pattern and all the force indentation curves were used during the anaylysis. In contrast, during each force volume experiment, some force indentation curves have to be excluded from analysis since they are performed on the flat surface. Furthermore, based on the observation made in the previous section, only force indentation curves performed in the vicinity of the pore center should be considered for analysis. After careful analysis of the force indentation curves (shown in the appendix of this chapter), only four force 
indentation curves performed in the middle of each pore were used to analyze the results. Histograms obtained from both experiments are compared in figure 3.44 .
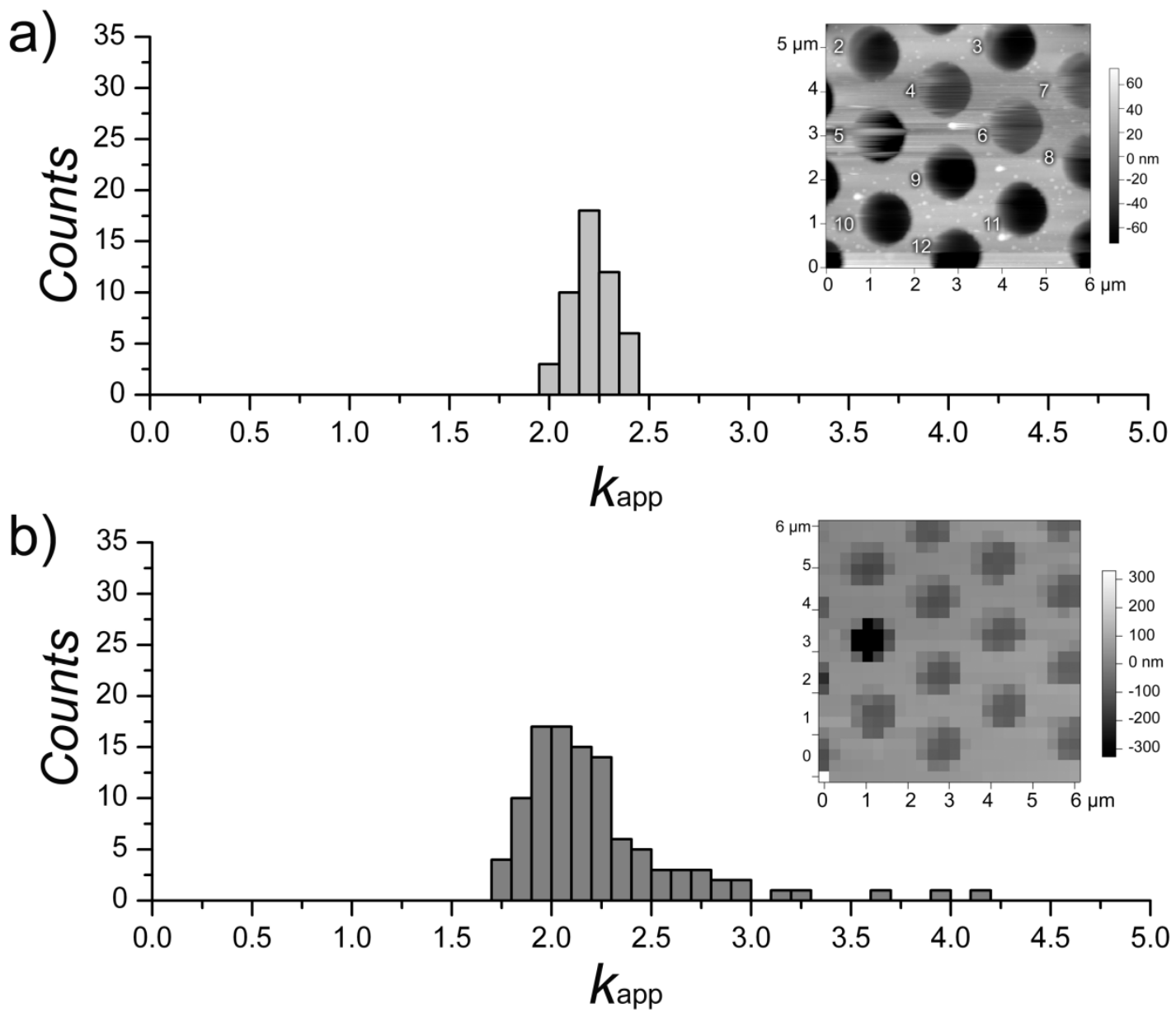

Figure 3.44 Comparison of $k_{\text {app }}$ obtained from (a) individual force indentation curves where $k_{\text {app }}: 2.22 \pm$ $0.12 \mathrm{mN} \mathrm{m}^{-1}$ and (b) from force volume images where $k_{\mathrm{app}}: 2.1 \pm 0.2 \mathrm{mN} \mathrm{m}^{-1}$

The average $k_{\text {app }}$ calculated from individual force indentation curves was of $2.22 \pm 0.12$ $\mathrm{mN} \mathrm{m}^{-1}$ while the average $k_{\text {app }}$ from force volume mapping was of $2.1 \pm 0.2 \mathrm{mN} \mathrm{m}^{-1}$. The relative error of $9.5 \%$ associated with force volume mapping is significantly higher in contrast to individual force indentation curves where the relative error is of $5.4 \%$. However, given the increased number of force curves that can be obtained from force volume experiments together with the visual result in the form of an image while still remaining within acceptable error range, force volume experiments have been judged a valuable alternative to individual force indentation curves. Force volume mapping has been used to investigate the effect of external variable on the mechanical response of pore-spanning membranes and is presented in the following section. 


\subsubsection{RELEASE OF TENSION BY ADDITION OF ISOPROPANOL}

Short chain alcohols are known mediators of bilayer tension playing an important role in many membrane processes such as fusion, drug delivery and general anesthesia. ${ }^{54}$ The suitability of the new pore-spanning membrane model system to conduct membrane alcohol exposure studies was investigated in the following experiment. Pore-spanning lipid membranes ware formed and subsequently, isopropanol was added to the measuring chamber. Force volume images of the membrane covered pores before and after addition of isopropanol were acquired.

The decrease of membrane tension was measured after the addition of short chain alcohols which are believed to reversibly insert in the head-group region of the membrane. Figure 3.45 shows typical force indentation and retraction (relaxation) curves performed on pore-spanning bilayers prepared from POPC GUV spreading on functionalized porous substrates. Increasing volume fraction of isopropanol was added to the buffer surrounding the sample.

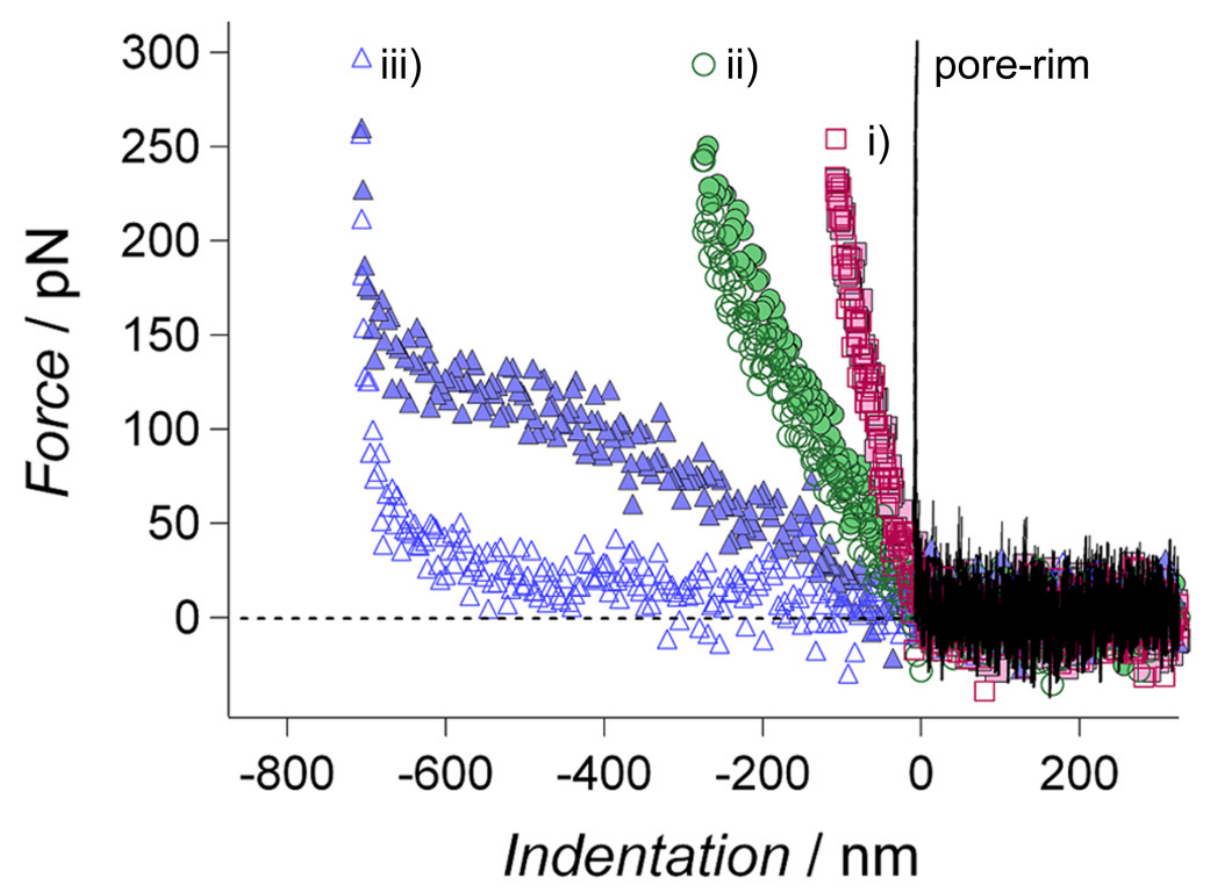

Figure 3.45 Typical force indentation curves obtained on (i) a pure POPC bilayer, (ii) on the same POPC bilayer in the presence of isopropanol $(0.67 \mathrm{M})$ and (iii) in the presence of $1.28 \mathrm{M}$ isopropanol. Hysteresis between the indentation (full markers) and relaxation (open markers) curves becomes more apparent with addition of isopropanol (ii versus iii)). 
The alteration of the mechanical response of the pore-spanning to the indentation is clearly distinguishable in figure 3.45 , where the $k_{\text {app }}$ decreases as the concentration of isoproanol is increased.

Values of $k_{\text {app }}$ calculated from the experiments performed on the pore-spanning membranes before and after addition of isopropanol are depicted in the histograms of figure 3.46. In comparison to the initial $1.6 \pm 0.3 \mathrm{mN} \mathrm{m}^{-1}$, and $0.6 \pm 0.2 \mathrm{mN} \mathrm{m}^{-1}$ in $0.67 \mathrm{M}$ isopropanol, a tension release of one order of magnitude, down to $0.19 \pm 0.07 \mathrm{mN} \mathrm{m}^{-1}$, was detected by force spectroscopy after addition of isopropanol (1.28 M).

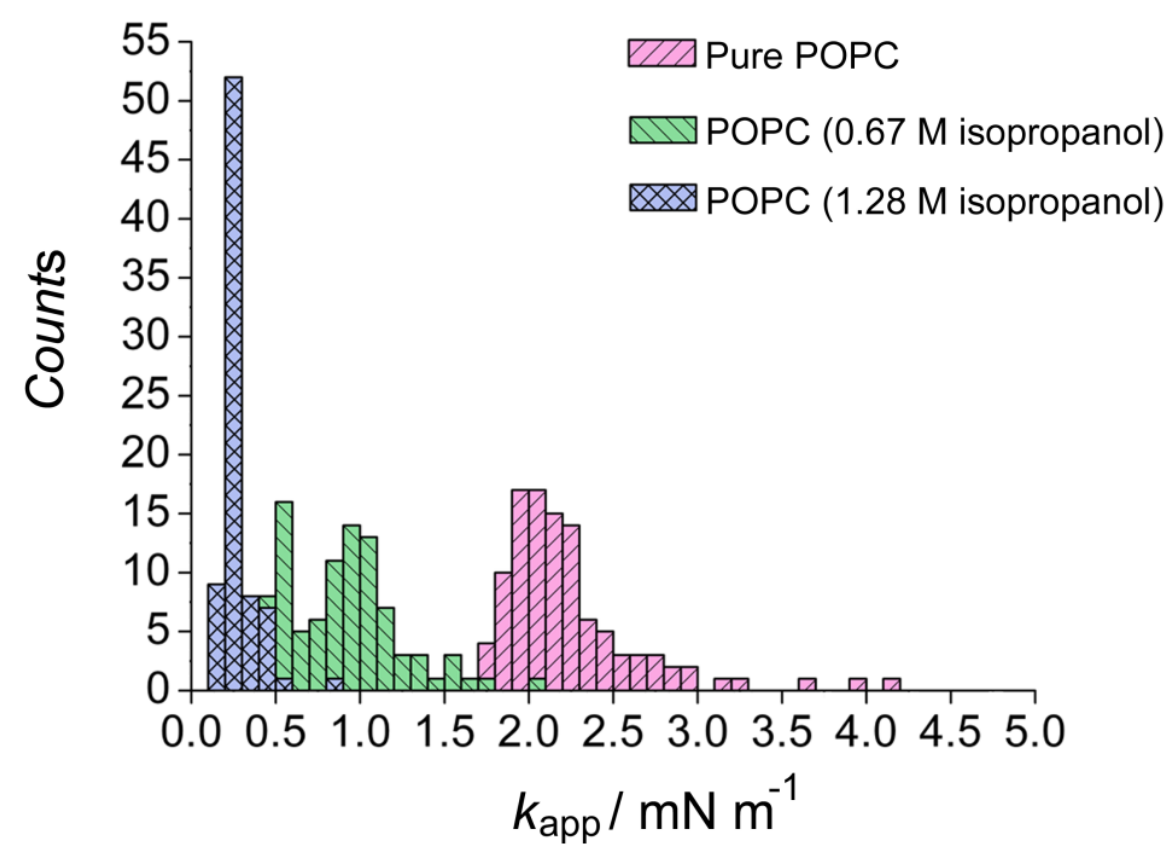

Figure 3.46 Histogram of $k_{\text {app }}$ shows the gradual release of tension as isopropanol is added to the POPC bilayer where $k_{\text {app }}$ POPC: $2.2 \pm 0.4 \mathrm{mN} \mathrm{m}^{-1}, k_{\text {app }}$ of POPC in $0.67 \mathrm{M}$ isopropanol: $0.9 \pm 0.3 \mathrm{mN} \mathrm{m}^{-1}$ and $k_{\text {app }}$ of POPC in $1.28 \mathrm{M}$ isopropanol: $0.3 \pm 0.1 \mathrm{mN} \mathrm{m}^{-1}$.

Conceptually, it is possible that isopropanol molecules partition preferentially into the free-standing bilayer due to its slightly dilated state and as a consequence reduce the residual pre-stress in the bilayer over the pores (Figure 3.47). 
a)

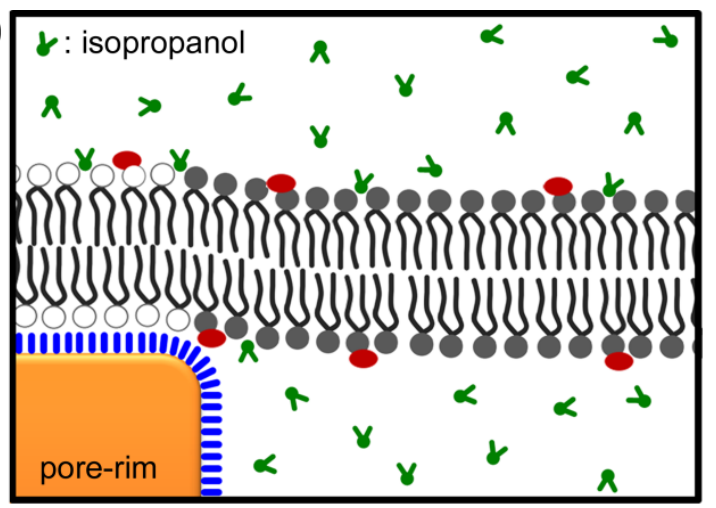

b)

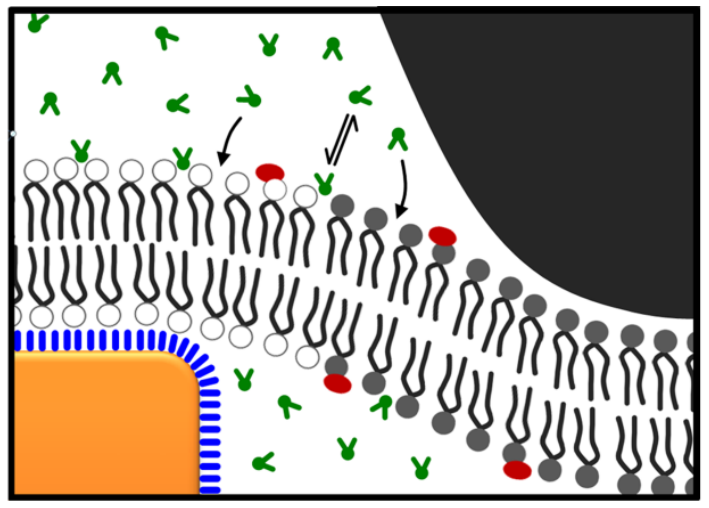

Figure 3.47 In (a), the interaction of isopropanol with the unperturbed pore-spanning lipid bilayer is shown with some of the isopropanol molecules (green) inserting into the lipid bilayer. In (b), the insertion of isopropanol into the bilayer is facilitated by stretching and moving the bilayer in response to indentation by the AFM tip as represented by lipids with hollow head groups moving into the center of the pore. Indentation is thus proposed to increase the spacing between lipid molecules, allowing for facilitated isopropanol insertion, a process which reverses on retraction of the AFM tip.

Interestingly, the addition of isopropanol also produces severe viscous losses inferable from the strong hysteresis between indentation and relaxation curves (Figure 3.45, iii). This hysteresis is attributed to either an increased inter-bilayer friction due to the presence of short chain alcohols that disturb the order of the leaflet structure or an increasing partitioning of the isopropanol into the bilayer during indentation of the bilayer (Figure $3.47 \mathrm{~b}$ ), an effect which is reversed upon the retraction of the AFM tip. The indentation and relaxation curves turned out to be reproducible and the influence of isopropanol on the membrane tension reversible.

\subsubsection{DETERMINATION OF BENDING MODULUS THROUGH TETHER ANALYSIS}

The bending modulus $\kappa$ of phospholipid bilayer membranes is a mechanical property which is challenging to obtain because of its relatively small values (typically between 10 and $100 \mathrm{k}_{\mathrm{b}} \mathrm{T}$ ) in comparison to thermal noise. ${ }^{55}$ In flicker spectroscopy, $\kappa$ is determined from thermally induced shape undulations of vesicles, while micropipette aspiration technique relies on the analysis of the relative change in area under aspiration pressure to calculate $\kappa$. These are two well established methods to determine $\kappa$ experimentally. ${ }^{16,56-58}$

Although these techniques provide reliable values, their use is restricted to very large vesicles and the $\kappa$ values obtained from such experiments describe global rather than local properties of the bilayer. Ultimately, local bending modules are desirable quantities but their access requires new experimental strategies. One approach would be to indent 
elastically decoupled pore-spanning bilayers. However, as it was mentioned in the previous section, the contribution of membrane bending to the mechanical response during indentation is negligible due to the large pore radius. Nevertheless, membrane bending information can be gained from force indentation experiments by analyzing membrane deformation during the retraction of the AFM tip. As the AFM tip is moved away from the surface, membrane tethers can form. These tethers are nanotubular structures that are pulled out of a bilayer as a result of applying localized forces. ${ }^{59}$ These hollow tubes persist between the AFM tip and the membrane until either their rupture is caused by depletion of lipid reservoir from which the tether is formed (i.e. bilayer membrane) or by loss of an adhesion point between the tether and the AFM tip. ${ }^{60,61}$ Because tether formation requires changes in the shape of the underlying membrane, the formation of such structures is strongly dependent on the membrane's mechanical properties. ${ }^{59}$ The force needed to pull a tether $F_{\text {tether }}$ depends on the intrinsic mechanical properties of the bilayer such as bending modulus, tension and shear viscosity.

Experimentally, tether formation is observed as a plateau of constant height in the retraction curve (Figure 3.48). The height of the plateau corresponds to the force required to pull out a tether out of a membrane.

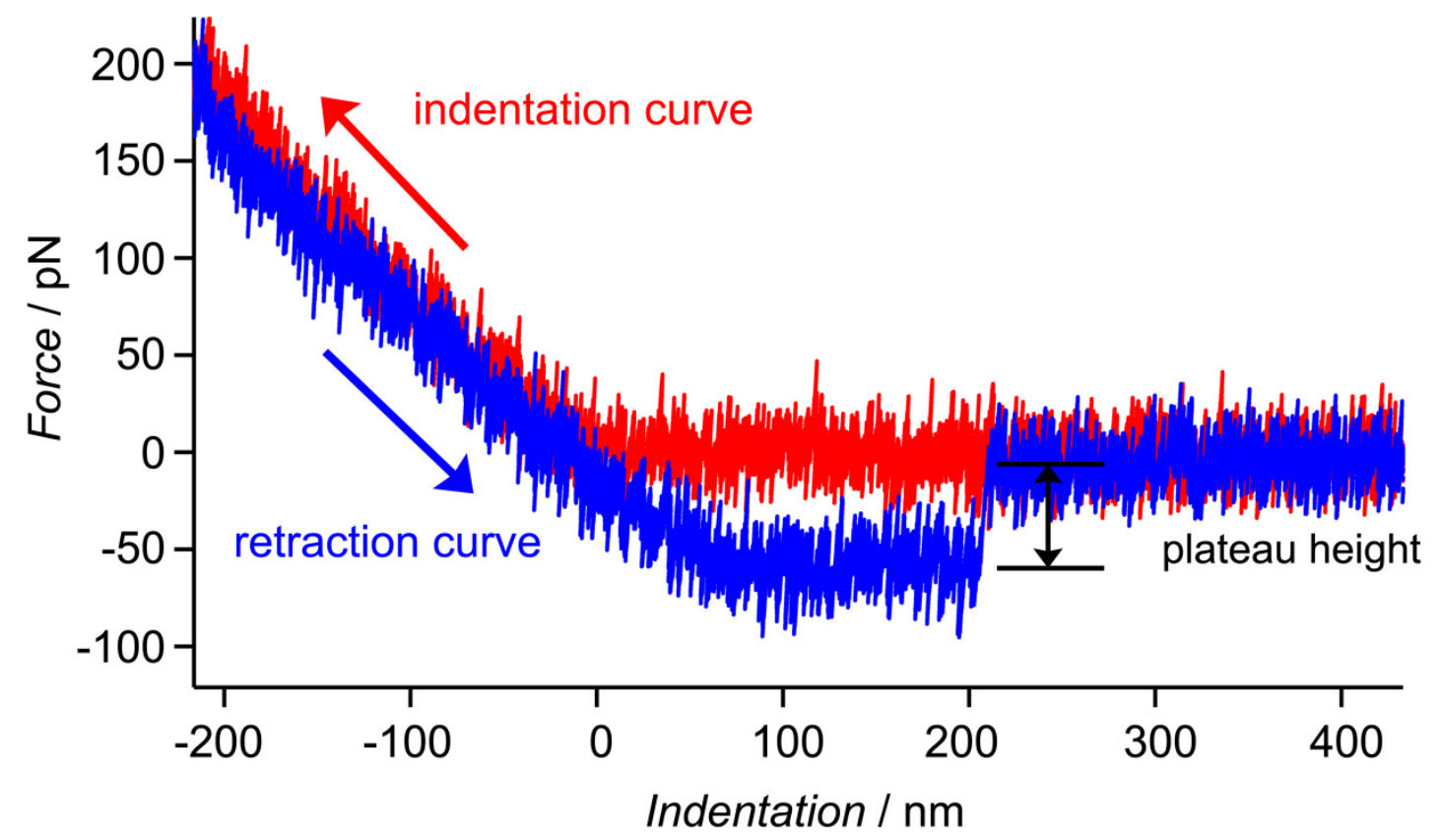

Figure 3.48 Typical force curve acquired during a force indentation experiment on a pore-spanning membrane showing an indentation of the membrane $\left(k_{\text {app }}\right.$ of $0.001 \mathrm{~N} / \mathrm{m}$ ) and a plateau (approx. $50 \mathrm{pN}$ in height) 
Tether formation of takes place during AFM tip retraction away from the surface, after tip-membrane interaction. Such adhesion interactions can be induced the by ligand/receptor approach, in which case the AFM tip is functionalized with ligands and the membrane is functionalized with receptors. Upon contact, the membrane can attach to the tip through the ligand/receptor interactions and a tether is pulled out. This approach was previously shown in colloidal force microscopy, optical and magnetic tweezer experiments. ${ }^{62-64}$ However, it has also been shown that tether formation can be initiated by prolonged (2-30s) contact of a non functionalized AFM tip with the membrane. ${ }^{65}$ Tether rupture is characterized by an instant decrease of tether force to zero, which appears as a sharp step in the retraction curve (Figure 3.48) and the height of this step represents $F_{\text {tether. }}$

In contrast to membrane indentation where the bending is negligible (Figure 3.49b), during tether formation, the membrane experiences a high bending at the anchoring point between the tip and the tether (Figure 3.49c).
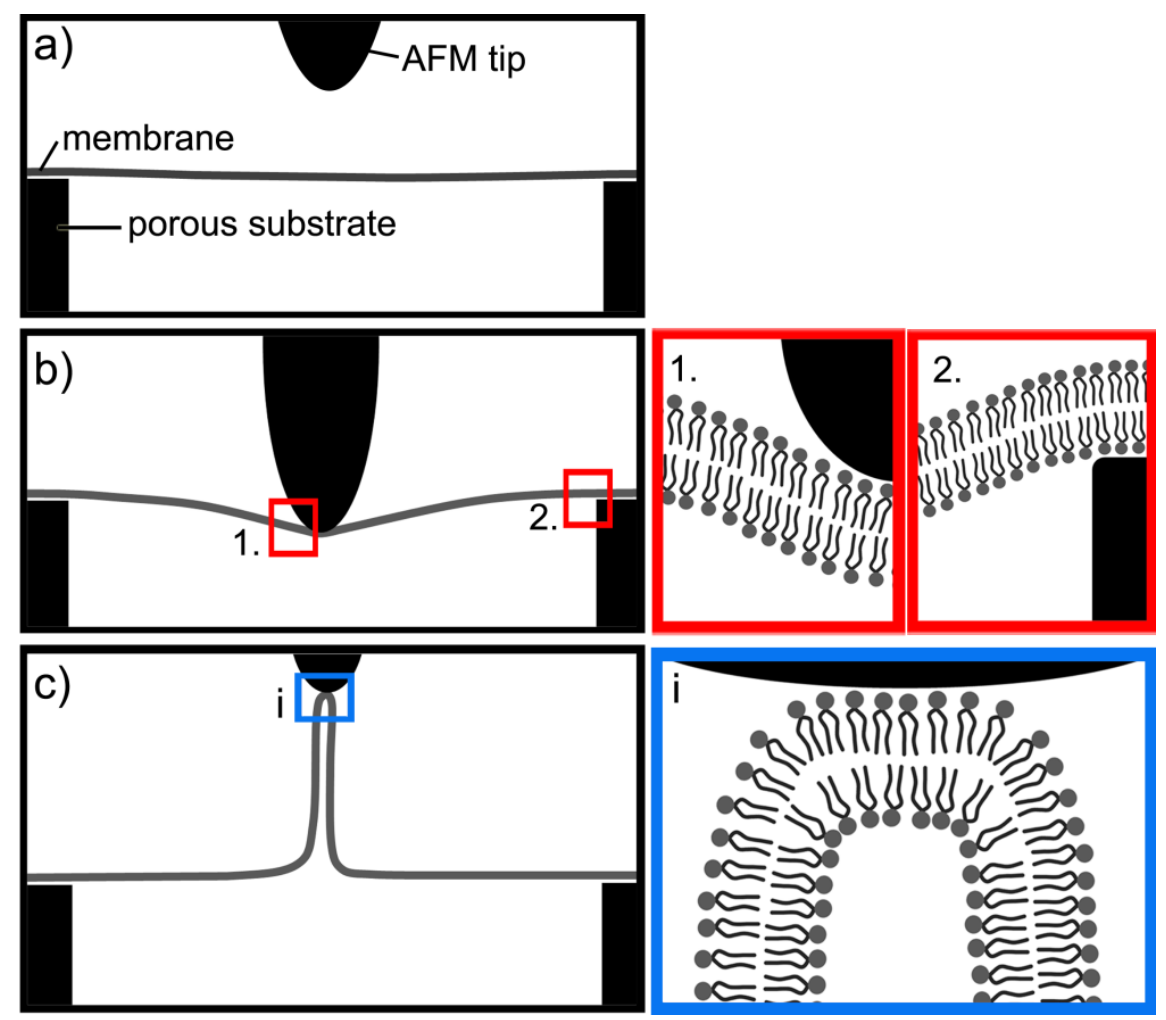

Figure 3.49 Schematic representation of an indentation experiment where (a) AFM tip is far away from the pore-spanning membrane, (b) AFM tip is indenting the membrane, red squares indicate areas where membrane experiences slight degree bending (c) a tether is pulled out as of the membrane as the AFM tip is retracted, square indicates area where membrane experiences high degree of bending. 
This fundamental difference between indentation and retraction experiments allows for quasi-simultaneous determination of membrane tension and membrane bending modulus form force indentation experiments followed by tip retraction from the surface. Membrane tension $\sigma$ is calculated using $k_{\text {app }}$ obtained from the slope of the indentation curve and bending modulus $\kappa$ can be calculated according to:

$$
\kappa=\frac{1}{\sigma}\left(\frac{F_{\text {tether }}}{2 \pi}\right)^{2}
$$

where $F_{\text {tether }}$ is force needed to pull out a tether. The inclusion of the viscous loss into the equation is omitted since it is too small $\left(>0.001 \mathrm{pN} \mathrm{s} \mu \mathrm{m}^{-1}\right)^{66}$ to be captured experimentally by AFM at velocities generally used for force indentation experiments $\left(0.5-5 \mu \mathrm{m} \mathrm{s}^{-1}\right)$.

Preliminary experiments were performed on fluid pore-spanning bilayer membranes with the goal to obtain both: $k_{\text {app }}$ from the indentation curves and plateau height $\left(F_{\text {tether }}\right)$ from the retraction curves. The experiments were performed such that the AFM tip (without any functionalization) indented the membrane and was kept in contact with it for $\sim 15$ seconds before it was retracted. From the few tethers that were observed on porespanning POPC lipid bilayer, the value of $\kappa$ was calculated following equation 3.11 and was of $2.8 \times 10^{-19} \mathrm{~J}$. Although the value of $\kappa$ pore-spanning POPC membrane is slightly higher than the values reported in literature $\left(\sim 1 \times 10^{-19} \mathrm{~J}\right),{ }^{67,68}$ it should be kept in mind that these are only preliminary results obtained from very few tethers. Furthermore, several tethers were also observed during the force indentation experiment where isopropanol was added to the measuring chamber containing pore-spanning POPC membranes (see Section 3.2.5.1). The value of $\kappa$ calculated from that experiment was of $1.09 \times 10^{-19} \mathrm{~J}$. The detection of $\kappa$ value change after addition of isopropanol to the environment of the pore-spanning membrane proves the effectiveness of the experimental approach based on the analysis of tether force in order to determine bending moduli of membranes. Additionally, the experimental result seem to follow the trend determined Ly et al. where the effect of short-chain alcohols on SOPC liposomes was monitored by micropipette aspiration experiments. ${ }^{69,70}$ Similarly to the result obtained here, Ly et al. 
showed that the bending modulus of the SOPC membranes decreases with increasing alcohol concentration.

In conclusion, the importance of the tether force analysis approach to determine $\kappa$ is that it allows for determination of local $\kappa$ values from pore-spanning membranes, a task which is not possible with flicker spectroscopy or micropipette aspiration experiments. These techniques probe entire surfaces of vesicles whereas force indentation curves can be performed on defined areas of a pore-spanning bilayer membrane. Furthermore, when combined with fluorescence microscopy and AFM imaging, force indentation curves can provide lateral membrane tension and bending modulus values with relation to protein content and distribution in the membrane. 


\subsubsection{APPENDIX}

Force volume experiments were performed on pore-spanning lipid membranes and the $k_{\text {app }}$ calculated from the force indentation curves were compared. Based on the results compiled in the table below, it was determined that based on the diameter of the pore (here $1200 \mathrm{~nm}$ ) and the average number of force curves performed across each pore (approximately 4), only the 4 curve performed in the middle of each pore should be considered for analysis.

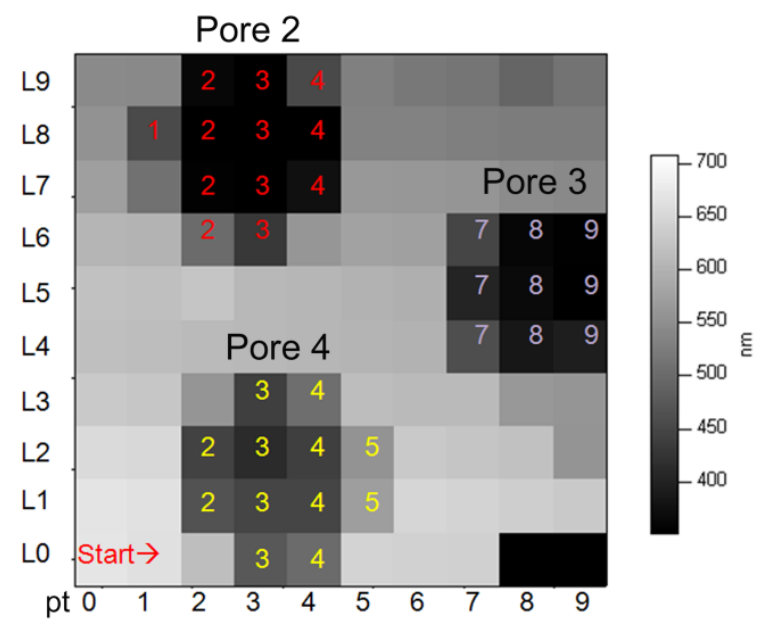

\begin{tabular}{|l|l|}
\multicolumn{2}{|c}{ Pore 2 } \\
\hline Slope (N/m) & Curve \\
\hline 0.00212 & L6_pt_2 \\
\hline 0.00179 & L6_pt_3 \\
\hline $\mathbf{0 . 0 0 1 3 7}$ & L7_pt_2 \\
\hline $\mathbf{0 . 0 0 1 2 5}$ & L7_pt_3 \\
\hline 0.00145 & L7_pt_4 \\
\hline 0.00259 & L8_pt_1 \\
\hline $\mathbf{0 . 0 0 1 3 6}$ & L8_pt_2 \\
\hline $\mathbf{0 . 0 0 1 2 7}$ & L8_pt_3 \\
\hline 0.00143 & L8_pt_4 \\
\hline 0.00170 & L9_pt_2 \\
\hline 0.00154 & L9_pt_3 \\
\hline 0.00140 & L9_pt_4 \\
\hline
\end{tabular}

Pore 3

Pore 4

\begin{tabular}{|l|l|l|l|}
\hline Slope (N/m) & Curve & Slope (N/m) & Curve \\
\hline 0.00290 & L4_pt_7 & 0.00160 & L0_pt_3 \\
\hline 0.00139 & L4_pt_8 & 0.00183 & L0_pt_4 \\
\hline 0.00140 & L4_pt_9 & 0.00156 & L1_pt_2 \\
\hline 0.00165 & L5_pt_7 & $\mathbf{0 . 0 0 1 2 9}$ & L1_pt_3 \\
\hline $\mathbf{0 . 0 0 1 2 8}$ & L5_pt_8 & $\mathbf{0 . 0 0 1 2 9}$ & L1_pt_4 \\
\hline $\mathbf{0 . 0 0 1 2 9}$ & L5_pt_9 & 0.00187 & L1_pt_5 \\
\hline 0.00229 & L6_pt_7 & 0.00156 & L2_pt_2 \\
\hline 0.00145 & L6_pt_8 & $\mathbf{0 . 0 0 1 3 1}$ & L2_pt_3 \\
\hline 0.00144 & L6_pt_9 & $\mathbf{0 . 0 0 1 4 0}$ & L2_pt_4 \\
\hline & & 0.00188 & L2_pt_5 \\
\hline & & 0.00170 & L3_pt_3 \\
\hline & & 0.00210 & L3_pt_4
\end{tabular}

Figure A3.1 Force volume image showing 3 membrane-covered pores. Each pore has been probed repetedly and the $k_{\text {app }}$ calculated from the slope of the indentation curves are shown in the tables below the image. The values of $k_{\text {app }}$ seem to be similar when the force curves are acquired in the middle of the pore. 


\subsection{REFERENCES}

1. Arifin, D. R.; Palmer, A. F., Polymersome encapsulated hemoglobin: A novel type of oxygen carrier. Biomacromolecules 2005, 6, (4), 2172-2181.

2. Dimova, R.; Seifert, U.; Pouligny, B.; Förster, S.; Döbereiner, H. G., Hyperviscous diblock copolymer vesicles. Eur. Phys. J. E 2002, 7, (3), 241-250.

3. Lee, J. C. M.; Bermudez, H.; Discher, B. M.; Sheehan, M. A.; Won, Y.-Y.; Bates, F. S.; Discher, D. E., Preparation, stability, and in vitro performance of vesicles made with diblock copolymers. Biotechnology and Bioengineering 2001, 73, (2), 135-145.

4. Massignani, M.; Lomas, H.; Battaglia, G., Polymersomes: A synthetic biological approach to encapsulation and delivery. In Modern techniques for nano- and microreactors/-reactions, Springer Berlin / Heidelberg: 2010; Vol. 229, pp 115-154.

5. Mills, J. K.; Needham, D., Targeted drug delivery. Expert Opinion on Therapeutic Patents 1999, 9, (11), 1499-1513.

6. Discher, B. M.; Won, Y.-Y.; Ege, D. S.; Lee, J. C. M.; Bates, F. S.; Discher, D. E.; Hammer, D. A., Polymersomes: Tough vesicles made from diblock copolymers. Science 1999, 284, (5417), 1143-1146.

7. Discher, D. E.; Eisenberg, A., Polymer vesicles. Science 2002, 297, (5583), 967-973.

8. Mabrouk, E.; Cuvelier, D.; Pontani, L. L.; Xu, B.; Levy, D.; Keller, P.; Brochard-Wyart, F.; Nassoy, P.; Li, M. H., Formation and material properties of giant liquid crystal polymersomes. Soft Matter 2009, 5, (9), 1870-1878.

9. Mueller, W.; Koynov, K.; Fischer, K.; Hartmann, S.; Pierrat, S.; Basche, T.; Maskos, M., Hydrophobic shell loading of PB-b-PEO vesicles. Macromolecules 2009, 42, (1), 357-361.

10. Boal, D., Mechanics of the cell. Cambridge University Press: Cambridge, 2001.

11. Mey, I.; Stephan, M.; Schmitt, E. K.; Muller, M. M.; Ben Amar, M.; Steinem, C.; Janshoff, A., Local membrane mechanics of pore-spanning bilayers. Journal of the American Chemical Society 2009, 131, (20), 7031-7039.

12. Liu, C.; Case, E., Effect of indenter tip radius on the load deflection behavior of thin plates. Experimental Mechanics 1997, 37, (2), 175-181.

13. Bermudez, H.; Hammer, D. A.; Discher, D. E., Effect of bilayer thickness on membrane bending rigidity. Langmuir 2004, 20, (3), 540-543.

14. Bhatia, N. M.; Nachbar, W., Finite indentation of an elastic membrane by a spherical indenter. International Journal of Non-Linear Mechanics 1968, 3, (3), 307-324.

15. Norouzi, D.; Muller, M. M.; Deserno, M., How to determine local elastic properties of lipid bilayer membranes from atomic-force-microscope measurements: A theoretical analysis. Physical Review E 2006, 74, (6), 061914-1-12.

16. Evans, E.; Rawicz, W., Entropy-driven tension and bending elasticity in condensed-fluid membranes. Physical Review Letters 1990, 64, (17), 2094.

17. Needham, D.; Hochmuth, R. M., Electro-mechanical permeabilization of lipid vesicles. Role of membrane tension and compressibility. Biophysical Journal 1989, 55, (5), 1001-1009. 
18. Goncalves, R. P.; Agnus, G.; Sens, P.; Houssin, C.; Bartenlian, B.; Scheuring, S., Two-chamber AFM: Probing membrane proteins separating two aqueous compartments. Nat Meth 2006, 3, (12), 1007-1012.

19. Zhang, C. Y.; Zhang, Y. W., Effects of membrane pre-stress and intrinsic viscoelasticity on nanoindentation of cells using afm. Philosophical Magazine 2007, 87, (23), 3415-3435.

20. Evans, E. A.; Hochmuth, R. M., Membrane viscoelasticity. Biophysical Journal 1976, 16, (1), 111.

21. Bermudez, H.; Brannan, A. K.; Hammer, D. A.; Bates, F. S.; Discher, D. E., Molecular weight dependence of polymersome membrane structure, elasticity, and stability. Macromolecules 2002, $35,(21), 8203-8208$.

22. Lee, C.; Wei, X.; Kysar, J. W.; Hone, J., Measurement of the elastic properties and intrinsic strength of monolayer graphene. Science 2008, 321, (5887), 385-388.

23. Collinsworth, A. M.; Zhang, S.; Kraus, W. E.; Truskey, G. A., Apparent elastic modulus and hysteresis of skeletal muscle cells throughout differentiation. Am J Physiol Cell Physiol 2002, 283, (4), C1219-1227.

24. Lee, J. C. M.; Santore, M.; Bates, F. S.; Discher, D. E., From membranes to melts, rouse to reptation: Diffusion in polymersome versus lipid bilayers. Macromolecules 2002, 35, (2), 323-326.

25. Bremmell, K. E.; Evans, A.; Prestidge, C. A., Deformation and nano-rheology of red blood cells: An AFM investigation. Colloids and Surfaces B-Biointerfaces 2006, 50, (1), 43-48.

26. Morone, N.; Fujiwara, T.; Murase, K.; Kasai, R. S.; Ike, H.; Yuasa, S.; Usukura, J.; Kusumi, A., Three-dimensional reconstruction of the membrane skeleton at the plasma membrane interface by electron tomography. Journal of Cell Biology 2006, 174, (6), 851-862.

27. Dai, J. W.; Sheetz, M. P., Membrane tether formation from blebbing cells. Biophysical Journal 1999, 77, (6), 3363-3370.

28. Sheetz, M. P., Cell control by membrane-cytoskeleton adhesion. Nature Reviews Molecular Cell Biology 2001, 2, (5), 392-396.

29. Schmitt, E. K.; Vrouenraets, M.; Steinem, C., Channel activity of ompf monitored in nano-blms. Biophysical Journal 2006, 91, (6), 2163-2171.

30. Fine, T.; Mey, I.; Rommel, C.; Wegener, J.; Steinem, C.; Janshoff, A., Elasticity mapping of apical cell membranes. Soft Matter 2009, 5, (17), 3262-3265.

31. Aronsson, B. O.; Lausmaa, J.; Kasemo, B., Glow discharge plasma treatment for surface cleaning and modification of metallic biomaterials. Journal of Biomedical Materials Research 1997, 35 , (1), 49-73.

32. Ulman, A., Formation and structure of self-assembled monolayers. Chemical Reviews 1996, 96, (4), 1533-1554.

33. Hamai, C.; Cremer, P. S.; Musser, S. M., Single giant vesicle rupture events reveal multiple mechanisms of glass-supported bilayer formation. Biophysical Journal 2007, 92, (6), 1988-1999.

34. Lipowsky, R.; Sackmann, E., Structure and dynamics of membranes. Elsevier: Amsterdam, 1995; Vol. 1A. 
35. Liebermann, T.; Knoll, W., Surface-plasmon field-enhanced fluorescence spectroscopy. Colloids and Surfaces a-Physicochemical and Engineering Aspects 2000, 171, (1-3), 115-130.

36. Mayer, C.; Stich, N.; Schalkhammer, T.; Bauer, G., Slide-format proteomic biochips based on surface-enhanced nanocluster-resonance. Fresenius Journal of Analytical Chemistry 2001, 371, (2), 238-245.

37. Vasilev, K.; Knoll, W.; Kreiter, M., Fluorescence intensities of chromophores in front of a thin metal film. Journal of Chemical Physics 2004, 120, (7), 3439-3445.

38. Axelrod, D.; Koppel, D. E.; Schlessinger, J.; Elson, E.; Webb, W. W., Mobility measurement by analysis of fluorescence photobleaching recovery kinetics. Biophysical Journal 1976, 16, (9), 1055-1069.

39. Bayerl, T. M.; Bloom, M., Physical-properties of single phospholipid-bilayers adsorbed to micro glass-beads - a new vesicular model system studied by ${ }^{2} \mathrm{H}$-nuclear magnetic-resonance. Biophysical Journal 1990, 58, (2), 357-362.

40. Chiantia, S.; Ries, J.; Kahya, N.; Schwille, P., Combined afm and two-focus sfcs study of raftexhibiting model membranes. ChemPhysChem 2006, 7, (11), 2409.

41. Koenig, B. W.; Kruger, S.; Orts, W. J.; Majkrzak, C. F.; Berk, N. F.; Silverton, J. V.; Gawrisch, K., Neutron reflectivity and atomic force microscopy studies of a lipid bilayer in water adsorbed to the surface of a silicon single crystal. Langmuir 1996, 12, (5), 1343-1350.

42. Kahya, N.; Scherfeld, D.; Bacia, K.; Poolman, B.; Schwille, P., Probing lipid mobility of raftexhibiting model membranes by fluorescence correlation spectroscopy. Journal of Biological Chemistry 2003, 278, (30), 28109-28115.

43. Sonnleitner, A.; Schütz, G. J.; Schmidt, T., Free brownian motion of individual lipid molecules in biomembranes. Biophysical Journal 1999, 77, (5), 2638-2642.

44. Stelzle, M.; Miehlich, R.; Sackmann, E., Two-dimensional microelectrophoresis in supported lipid bilayers. Biophysical Journal 1992, 63, (5), 1346-1354.

45. Wagner, M. L.; Tamm, L. K., Tethered polymer-supported planar lipid bilayers for reconstitution of integral membrane proteins: Silane-polyethyleneglycol-lipid as a cushion and covalent linker. Biophysical Journal 2000, 79, (3), 1400.

46. Weiskopf, D.; Schmitt, E. K.; Kluhr, M. H.; Dertinger, S. K.; Steinem, C., Micro-BLMs on highly ordered porous silicon substrates: Rupture process and lateral mobility. Langmuir 2007, 23, (18), 9134-9139.

47. Lazzara, T. D.; Kliesch, T. T.; Janshoff, A.; Steinem, C., Orthogonal functionalization of nanoporous substrates: Control of 3D surface functionality. ACS Appl. Mater. Interfaces 2011, In Press.

48. Dietrich, C.; Bagatolli, L. A.; Volovyk, Z. N.; Thompson, N. L.; Levi, M.; Jacobson, K.; Gratton, E., Lipid rafts reconstituted in model membranes. Biophysical Journal 2001, 80, (3), 1417-1428.

49. Stottrup, B. L.; Veatch, S. L.; Keller, S. L., Nonequilibrium behavior in supported lipid membranes containing cholesterol. Biophysical Journal 2004, 86, (5), 2942-2950.

50. Sackmann, E., Supported membranes: Scientific and practical applications. Science 1996, 271, (5245), 43-48. 
51. Kocun, M.; Mueller, W.; Maskos, M.; Mey, I.; Geil, B.; Steinem, C.; Janshoff, A., Viscoelasticity of pore-spanning polymer membranes derived from giant polymersomes. Soft Matter 2010, 6, (11), 2508-2516.

52. Steltenkamp, S.; Muller, M. M.; Deserno, M.; Hennesthal, C.; Steinem, C.; Janshoff, A., Mechanical properties of pore-spanning lipid bilayers probed by atomic force microscopy. Biophysical Journal 2006, 91, (1), 217-226.

53. Niggemann, G.; Kummrow, M.; Helfrich, W., The bending rigidity of phosphatidylcholine bilayers - dependences on experimental-method, sample cell sealing and temperature. Journal de Physique II: Atomic, Molecular and Cluster Physics, Chemical Physics, Mechanics and Hydrodynamics 1995, 5, (3), 413-425.

54. Delanoe-Ayari, H.; Al Kurdi, R.; Vallade, M.; Gulino-Debrac, D.; Riveline, D., Membrane and acto-myosin tension promote clustering of adhesion proteins. Proceedings of the National Academy of Sciences of the United States of America 2004, 101, (8), 2229-2234.

55. Cuvelier, D.; Derényi, I.; Bassereau, P.; Nassoy, P., Coalescence of membrane tethers: Experiments, theory, and applications. Biophysical Journal 2005, 88, (4), 2714-2726.

56. Mitov, M. D.; Meleard, P.; Winterhalter, M.; Angelova, M. I.; Bothorel, P., Electric-fielddependent thermal fluctuations of giant vesicles. Physical Review E 1993, 48, (1), 628.

57. Peterson, M. A., Linear response of the human erythrocyte to mechanical stress. Physical Review A 1992, 45, (6), 4116.

58. Rawicz, W.; Olbrich, K. C.; McIntosh, T.; Needham, D.; Evans, E., Effect of chain length and unsaturation on elasticity of lipid bilayers. Biophysical Journal 2000, 79, (1), 328-339.

59. Hosu, B. G.; Sun, M.; Marga, F.; Grandbois, M.; Forgacs, G., Eukaryotic membrane tethers revisited using magnetic tweezers. Physical Biology 2007, 4, (2), 67-78.

60. Waugh, R. E.; Song, J.; Svetina, S.; Zeks, B., Local and nonlocal curvature elasticity in bilayermembranes by tether formation from lecithin vesicles. Biophysical Journal 1992, 61, (4), 974-982.

61. Hochmuth, F. M.; Shao, J. Y.; Dai, J.; Sheetz, M. P., Deformation and flow of membrane into tethers extracted from neuronal growth cones. Biophys $J$ 1996, 70, (1), 358-69.

62. Lorenz, B.; Keller, R.; Sunnick, E.; Geil, B.; Janshoff, A., Colloidal probe microscopy of membrane-membrane interactions: From ligand-receptor recognition to fusion events. Biophysical Chemistry 2010, 150, (1-3), 54-63.

63. Dai, J.; Sheetz, M. P., Mechanical properties of neuronal growth cone membranes studied by tether formation with laser optical tweezers. Biophysical Journal 1995, 68, (3), 988-996.

64. Edmondson, K. E.; Denney, W. S.; Diamond, S. L., Neutrophil-bead collision assay: Pharmacologically induced changes in membrane mechanics regulate the PSGL-1/P-selectin adhesion lifetime. Biophysical Journal 2005, 89, (5), 3603-3614.

65. Sun, M.; Graham, J. S.; Hegedüs, B.; Marga, F.; Zhang, Y.; Forgacs, G.; Grandbois, M., Multiple membrane tethers probed by atomic force microscopy. Biophysical Journal 2005, 89, (6), 43204329.

66. Evans, E.; Yeung, A., Hidden dynamics in rapid changes of bilayer shape. Chemistry and Physics of Lipids 1994, 73, (1-2), 39-56. 
67. Bouvrais, H.; Meleard, P.; Pott, T.; Jensen, K. J.; Brask, J.; Ipsen, J. H., Softening of popc membranes by magainin. Biophysical Chemistry 2008, 137, (1), 7-12.

68. Kucerka, N.; Tristram-Nagle, S.; Nagle, J. F., Structure of fully hydrated fluid phase lipid bilayers with monounsaturated chains. Journal of Membrane Biology 2005, 208, (3), 193-202.

69. Ly, H. V.; Block, D. E.; Longo, M. L., Interfacial tension effect of ethanol on lipid bilayer rigidity, stability, and area/molecule: A micropipet aspiration approach. Langmuir 2002, 18, (23), 89888995.

70. Ly, H. V.; Longo, M. L., The influence of short-chain alcohols on interfacial tension, mechanical properties, area/molecule, and permeability of fluid lipid bilayers. Biophysical Journal 2004, 87, (2), 1013-1033. 


\section{CONCLUSION AND OUTLOOK}

The free-standing character of the pore-spanning polymer and lipid membranes that were prepared allow for the study of membrane mechanical properties by indentation experiments which are unrestricted by the underlying substrate. The simple and reliable preparation techniques of solvent-free, mobile, robust, pore-spanning membranes were described in this thesis.

In contrast to pore-spanning membrane preparation techniques, where the membrane is reconstituted by painting lipid or polymers dissolved in an organic solvent over pore apertures, ${ }^{1,2}$ the pore-spanning membranes prepared in this work have been formed by giant vesicle rupture. Pore-spanning membranes formed in this manner offer the advantages of being solvent-free, not tethered to the surface and reflect the composition of the vesicles.

Atomic force microscopy (AFM) force indentation results were used to elucidate mechanical properties of the membranes. AFM force indentation experiments are an alternative to micropipette aspiration technique since the latter technique is limited to entire vesicle studies. More importantly, AFM indentation experiments can be performed at defined areas on the pore-spanning membranes, which allows the extraction of local mechanical constants of the membrane in comparison to the global values obtained form micropipette aspiration experiments of whole vesicles. Additionally, the use of substrates with a defined pore array allows for membrane decoupling and the possibility of performing of multiple experiments on a single substrate. Force volume experiments generate images based on the mechanical response of the membrane to indentation in addition to collecting hundreds of force indentation curves in a short time frame.

Force indentation experiments on pore-spanning membranes prepared from PB- $b$-PEO polymersomes provided membrane mechanical constants such as area compressibility, elastic modulus and energy dissipation. Chemical modification of the polymer membrane through cross-linking of the PB hydrophobic core and the subsequent study of modification effects was performed. The results showed that polymer chain entanglement was responsible for membrane viscoelasticity and confirmed that the AFM force indentation experiments can be a reliable tool to investigate membrane mechanics. 
Moreover, pore-spanning lipid membranes can be used as model systems where the porous scaffold mimics the effect of the cytoskeleton found in native plasma membranes. A two step functionalization procedure of the porous substrates was developed to successfully prepare solvent-free, laterally mobile, robust pore-spanning phospholipid membranes by giant phospholipid vesicle rupture. The sensitivity of the AFM force indentation experiments to discern changes in the mechanical properties of pore-spanning membranes was shown by measurement of membrane lateral tension before and after addition of isopropanol to the membrane environment. Membrane tension release of about one order of magnitude was detected after increasing the isopropanol content of the aqueous environment to $1.28 \mathrm{M}$. Additionally, preliminary results providing membrane bending modulus were obtained by membrane tether formation.

As it has been shown here, vesicles can be forcefully ruptured over porous substrates in order to use the pore openings as probing zones. In particular, the pore-spanning preparation procedure and the subsequent mechanical investigation could be applied to study the recently prepared giant phospholipid/block copolymer hybrid vesicles. ${ }^{3}$ Although micropipette aspiration experiments were performed on these lipopolymersomes, the pore-spanning membrane and AFM force indentation approach proposed in this thesis could yield information about membrane tension and bending with respect to the exact membrane composition at a given location. Such precise information could be instructive in terms of further incorporation of membrane components into these hybrid vesicles. Furthermore, investigation of gold nanoparticle-loaded phosphatidylcholine vesicles where nanoparticles aggregate in a Janus fashion ${ }^{4}$ could benefit from a localized force indentation experiments to provide information about the nanoparticle-induced changes in the mechanics of the membrane.

In terms of more biologically relevant studies, Shchelkovskyy et al. recently reported on the membrane bending rigidity decrease with increasing concentration of HIV-1 fusion protein in phospholipid membranes. ${ }^{5}$ The authors have reported deviations from average values which they attributed to inhomogeneous distribution of the peptide in the vesicular membranes. By investigating fusion protein containing-membranes by AFM imaging and force indentation experiments, one could possibly detect the presence of protein aggregates and perform mechanical investigation experiments in both, protein poor and protein rich areas of the membrane in order to determine the effect of protein aggregation on membrane bending modulus. 
In conclusion, the formation of solvent-free pore-spanning membranes formed by giant vesicle rupture was discussed in this work. Pore-spanning membrane formation was controlled by varying external stimuli. In the case of polymer vesicles, osmotic stress induced vesicle rupture. Liposome rupture was performed by tailored porous substrate functionalization. Although the formation of pore-spanning membranes by vesicle rupture demands optimization of external stimuli to induce vesicle rupture and formation of porespanning membranes, the prepared membranes offer the benefits of being solvent-free and are not tethered to the porous support, furthermore, the membranes reflect the composition of the vesicles used for their formation. In addition, characterization of porespanning membranes through AFM imaging and force indentation experiments was proven to be an experimental approach sensitive enough to investigate subtle changes in the mechanical properties of the pore-spanning membranes. 


\section{REFERENCES}

1. Schmitt, E. K.; Nurnabi, M.; Bushby, R. J.; Steinem, C., Electrically insulating pore-suspending membranes on highly ordered porous alumina obtained from vesicle spreading. Soft Matter 2008, 4, (2), 250-253.

2. Rein, C.; Pszon-Bartosz, K.; Stibius, K. B.; Bjornholm, T.; Helix-Nielsen, C., Free-standing biomimetic polymer membrane imaged with atomic force microscopy. Langmuir 2011, 27, (2), 499-503.

3. Nam, J.; Beales, P. A.; Vanderlick, T. K., Giant phospholipid/block copolymer hybrid vesicles: Mixing behavior and domain formation. Langmuir 2011, 27, (1), 1-6.

4. Rasch, M. R.; Rossinyol, E.; Hueso, J. L.; Goodfellow, B. W.; Arbiol, J.; Korgel, B. A., Hydrophobic gold nanoparticle self-assembly with phosphatidylcholine lipid: Membrane-loaded and janus vesicles. Nano Letters 2010, 10, (9), 3733-3739.

5. Shchelokovskyy, P.; Tristram-Nagle, S.; Dimova, R., Effect of the HIV-1 fusion peptide on the mechanical properties and leaflet coupling of lipid bilayers. New Journal of Physics 2011, 13, 16. 


\section{MARTA KOCUN}

Citizenship: Canada

marta.kocun@gmail.com

- Education

DATE

Georg-August University Göttingen, Germany

2008-2011

Ph.D. Chemistry "Mechanical properties of pore-spanning membranes prepared from giant vesicles"

GGNB Program - Physics of biological and complex systems

Göttingen, Germany

Supervisor Prof. Dr. Andreas Janshoff

Concordia University, Canada

M.Sc. Chemistry "Single molecule force measurements of chitosan"

2006-2008

Montréal, Québec, Canada

Supervisors Dr. Louis Cuccia and Dr. Michel Grandbois

Concordia University, Canada

2001-2005

B.Sc. Specialization in Biochemistry

Montréal, Québec, Canada

Cegep André-Laurendeau, Canada

1999-2001

International Baccalaureate Diploma in Health Sciences, College diploma

Montréal, Québec, Canada

\section{- AWARDS}

GGNB Bridging fund, 2011 (5000 \$)

Best poster prize at the EBSA European Biophysics Congress, Genova, Italy, 2009

International Max Planck Research School Scholarship, Göttingen, Germany, 2009-2010 (50000 \$)

International Max Planck Research School Scholarship, Mainz, Germany, 2008 (20000 \$)

Best poster prize at $10^{\text {th }}$ Chemistry \& Biochemistry Graduate Conference, Montreal, Canada, 2007

SigmaXi Grant-in-Aid of research, 2006 (1000 \$)

\section{- RESEARCH INTEREST}

- Polymer self-assembly

- Atomic force microscopy characterization of surfaces and thin films

- Force indentation and single molecule force spectroscopy

- Study of porous interfaces and pore-spanning membranes (lipid and polymer) 


\section{- Publications}

Marta Kocun, Thomas D. Lazzara, Claudia Steinem, Andreas Janshoff (2011) Preparation of solvent-free, pore-spanning lipid bilayers to model the low tension in plasma membranes, accepted

Thomas D. Lazzara, Christian Carnarius, Marta Kocun, Andreas Janshoff, Claudia Steinem (2011) Pore-spanning membranes on nanoporous alumina, submitted

Marta Kocun, Michel Grandbois, Louis A. Cuccia (2011) Single molecule atomic force microscopy and force spectroscopy of chitosan Colloid Surf. B-Biointerfaces 82, 470-476.

Marta Kocun, Waltraut Mueller, Michael Maskos, Ingo Mey, Burkhard Geil, Claudia Steinem, Andreas Janshoff (2010) Viscoelasticity of pore-spanning polymer membranes derived from giant polymersomes Soft Matter 6, 2508-2516.

\section{- Conferences}

$8^{\text {th }}$ Hellenic Polymer Society Symposium, Crete, Greece, Poster presentation, (October 2010)

Frontiers of Chemistry: From Molecules to Systems, Paris, France, Poster presentation, (May 2010)

Workshop German Biophysical Society, Gomadingen, Germany, Poster presentation, (March 2010)

EBSA European Biophysics Congress, Genova, Italy, Poster presentation, (July 2009)

SPM and Organic Materials XVII, Bremen, Germany, Oral presentation, (June 2009)

$10^{\text {th }}$ CBGRC, Montreal, Canada, Poster presentation, (November 2007)

CSACS Student Symposium, Montreal, Canada, Oral presentation (September 2007)

ACS $234^{\text {th }}$ National Meeting \& Exposition, Boston, USA, Poster presentation (August 2007)

\section{- Teaching and Professional Experience}

Atomic Force Microscopy courses for graduate students, Göttingen University $\quad$ 2009-2010

$\begin{array}{ll}\text { Master student } & \text { 2006-2008 }\end{array}$

Teaching assistant, General Chemistry tutorials, Concordia University 2006-2008

Lab demonstrator: General Chemistry/Organic Chemistry, Concordia University 2006-2008

Research assistant, Concordia University, Dr. L. Cuccia Summer 2005

Research assistant, Concordia University, Dr. L. Cuccia Summer 2004

Internship, Structural and Functional Genomics, Concordia University Summer 2003

\section{- VOLUNTEER WORK}

$9^{\text {th }}$ and $10^{\text {th }}$ Chem and Biochem Graduate Conferences, organization committee $2006-2007$ CSACS Student Symposium, organization committee 2006

Expo Science Fair, demonstration booth 2005

\section{- LANGUAges}

English (native) / French (native) / Polish (native) / German (intermediate) / Spanish (beginner) 
